\title{
The amyloid beta peptide: a chemist's perspective. Role in Alzheimer's and fibrillization
}

Article

Accepted Version

Hamley, I. W. (2012) The amyloid beta peptide: a chemist's perspective. Role in Alzheimer's and fibrillization. Chemical Reviews, 112 (10). pp. 5147-5192. ISSN 1520-6890 doi: https://doi.org/10.1021/cr3000994 Available at https://centaur.reading.ac.uk/30230/

It is advisable to refer to the publisher's version if you intend to cite from the work. See Guidance on citing.

To link to this article DOI: http://dx.doi.org/10.1021/cr3000994

Publisher: American Chemical Society

All outputs in CentAUR are protected by Intellectual Property Rights law, including copyright law. Copyright and IPR is retained by the creators or other copyright holders. Terms and conditions for use of this material are defined in the End User Agreement.

www.reading.ac.uk/centaur

\section{CentAUR}


Central Archive at the University of Reading

Reading's research outputs online 
The Amyloid Beta Peptide: A Chemist's Perspective

Role in Alzheimer's and Fibrillization

I.W.Hamley

Dept of Chemistry, University of Reading, Whiteknights, Reading RG6 6AD, UK

I.W.Hamley@ reading.ac.uk

For Chemical Reviews. Revised 7/5/12. 


\section{CONTENTS}

1. INTRODUCTION

2. BIOLOGICAL, BIOCHEMICAL AND NEUROCHEMICAL

CHARACTERISTICS OF AD, INVOLVING A $\beta$ and APP

2.1 Genetic Markers for AD

2.1.1 Genetic Risk Factors

2.1.2 Transgenic Mice

$2.2 \mathrm{~A} \beta$ in vivo

2.2.1 Characteristics of $A \beta$ in vivo

2.2.2 Modelling AD and $\mathrm{A} \beta$ deposition in Other Organisms

2.2.3 APP and the Production of A $\beta$

2.2.4 A $\beta$ Mutations

2.3 Neuronal Toxicity of $A \beta$

$2.4 \quad$ Biomarkers for AD

2.5 Antibodies to $A \beta$ and Sequences Therein

2.6 Oligomers

2.6.1 Toxicity of Oligomers

2.6.2 Types of Oligomers

2.7 Ion Channel Hypothesis

2.8 Interactions of $A \beta$ with tau

3. THERAPEUTIC TREATMENTS

3.1 Existing Treatments 
3.2 Inhibitors of Fibrillization/Oligomerization

3.2.1 Small Molecules

3.2.2 Proteins and Particles

3.3 Inhibitors of the Secretase Enzymes

3.4 Immunization

3.5 Other Approaches

4. BIOPHYSICAL CHEMISTRY OF A $\beta$ AND FRAGMENT PEPTIDESSTRUCTURE AND AGGREGATION

4.1 A $\beta$ Conformation and Structure of Fibrils

4.2 Computer Simulations of A $\beta$ Conformation and Aggregation

4.3 Kinetics and Mechanisms of Fibrillization

4.3.1 Mechanisms

4.3.2 Kinetics

4.3.3 Thermodynamics

4.3.4 Lack of Sequence Specificity in A $\beta$ Aggregation

4.4 Polymorphism

4.5 Fragments

4.6 Micelles

4.7 Interactions with Lipid Membranes

4.8 Effect of Metal Ions

4.9 Conjugates of $A \beta$ with Polymers and Lipid Chains

5. SUMMARY AND OUTLOOK 


\section{INTRODUCTION}

This review is concerned with the role of fibrillization of the amyloid $\beta(\mathrm{A} \beta)$ peptide in Alzheimer's disease (AD). The perspective is that of a physical chemist and one aim is to introduce relevant key findings on physico-chemical properties. However, in addition, key aspects of the biology and biochemistry associated with the role of $A \beta$ in $\mathrm{AD}$ are also summarized (more detailed reviews of these aspects can be found elsewhere), ${ }^{1}$ as are developments in potential therapies and biomarkers.

The aggregation of the amyloid $\beta$ peptide into oligomers or fibrils is now implicated as a key process associated with progression of $\mathrm{AD} .^{2}$ This is the focus of the current review. Whilst the protein tau has an important role in AD progression, its processing occurs downstream of $A \beta$ accumulation. ${ }^{3}$ A marked decrease or absence of tau expression appears to reduce the neurotoxic effects of A $\beta .{ }^{4}$ Proteins including NAC (non-beta-amyloid component) are also co-deposited along with $\mathrm{A} \beta$ in plaques. ${ }^{5}$ NAC comprises residues $61-95$ of $\alpha$-synuclein, which is involved in amyloidoses with Lewy bodies such as Parkinson's disease. These topics are not discussed further herein, with the exception of a brief discussion (Section 2.8) of the interaction between $\mathrm{A} \beta$ and tau.

Due to the very large number of papers on $A \beta$, this review cannot be exhaustive in the space available. We have attempted to focus on key papers, and work that illustrates the main features of the subjects in the following sections. We have attempted to review work by many groups who have made important contributions. As there are also a large number of previous reviews on the topic of 
A $\beta$ aggregation and its relationship to neurodegenerative disease, we can also only cite a number of the key earlier reviews here. ${ }^{1,6}$

$\mathrm{AD}$ is the most common cause of dementia (representing around $50-80 \%$ of all cases ${ }^{7}$ ) with an estimated 18 million people worldwide currently affected by the condition (according to the World Health Organization). ${ }^{8}$ Its incidence increases dramatically with age, and the number of people with dementia is set to double in the next twenty years. ${ }^{7}$ The annual cost of dementia in the UK is estimated at $£ 23$ billion per annum including care and healthcare costs and lost productivity, which equates to £28k per patient. ${ }^{9}$ Alzheimer's disease accounts for about $70 \%$ of all late-onset dementia cases. ${ }^{10}$ Most cases occur relatively late in life, although around 5\% occurs in patients under 60 years old. These cases are termed early onset familial Alzheimer's disease (FAD). Genetic mutations have been linked to these conditions as discussed in section 2.1 below. In $\mathrm{AD}$, neurodegeneration is estimated to start $10-30$ years before clinical symptoms are detected. $^{2 \mathrm{a}, 11}$

Intense research activity is focussed on the development of treatments for Alzheimer's disease (AD) as discussed in Section 3. Several existing treatments can manage the condition but they do not arrest or reverse the progression of $\mathrm{AD}$, i.e. there is no cure. A healthy diet and exercise may contribute to reduced AD risk as might enhanced mental activity and social engagement. ${ }^{12}$ Calorie restriction and intermittent fasting also ameliorate age-related behavioural deficits in transgenic mice. ${ }^{13}$ Further discussion of these epidemiological studies is outside the scope of the present review. A number of strategies to treat the condition are actively being pursued by research teams in academia and the pharmaceutical industry. ${ }^{12 a, 14}$ These 
include (i) development of $\gamma$-secretase inhibitors $(\gamma$-secretase is an enzyme involved in cleavage of amyloid $\beta$ (A $\beta$ ) peptides from the Amyloid Precursor Protein), (ii) passive immunization based on $\mathrm{A} \beta$ antibodies (iii) inhibition of aggregation of oligomers. These are discussed further in Section 3.

Susceptibility to AD increases with aging, as indicated by large population screening studies and studies using monkeys ${ }^{15}$ and transgenic mice. ${ }^{15}$ Much research has focussed on early-onset $\mathrm{AD}$ for which genetic markers and the role of $\mathrm{A} \beta$ are readily identified. $^{2 \mathrm{c}}$ Table 1 shows characteristics of early-onset AD (EOAD). It is responsible for $\sim 2 \%$ of cases and can occur as early as 30 years of age. ${ }^{2 c}$ Late-onset $\mathrm{AD}$ (LOAD) is the more common variant that causes the majority of the cases of agedependent dementia. Age is the single biggest known risk factor, with the incidence of the disease increasing from approximately one in ten of those over 65, doubling roughly every five years to affect approximately half of individuals over $85 .^{2 \mathrm{c}, 8,16}$ Susceptibility to LOAD also seems to have a genetic basis, although a single genetic determinant does not exist - several genes associated with susceptibility to the condition are known (as discussed further in Section 2.1) and a combination of genes may also be involved. The progression of AD is similar for EOAD and LOAD and is arbitrarily divided into early/mild, moderate and severe cases.

Oxidative stress may play an important role in the age-dependent susceptibility to AD. ${ }^{17}$ Oxidative stress involves the production of free radicals (especially hydroxyl radicals) in the presence of metal ions, which can influence metabolism, and also promote $A \beta$ aggregation, the latter subject being discussed further in section 4.8. The free radicals can cause increased lipid peroxidation, and the formation of associated 
byproducts, as well as protein and DNA oxidation in the AD brain. Diminished mitochondrial energy metabolism may play a role in AD pathogenesis, due at least in part to reduced cyclooxygenase (COX) activity (section 2.1). ${ }^{17}$ Excitotoxicity is the overstimulation of $\mathrm{N}$-methyl-D-aspartate (NMDA) or 2-amino-3-(5-methyl-3-oxo-1,2oxazol-4-yl)propanoic acid (AMPA) receptors (NMDARs and AMPARs respectively) by glutamate or aspartate, leading to neuronal hyperexcitability and death. It can also generate excess reactive oxygen species (ROS). The role of oxidative stress is evidenced by the presence of protein glycation end products in $\mathrm{A} \beta$ aggregates, as well as an increase in the number of activated microglial cells (section 2.3). ${ }^{17}$ The inflammation that results from oxidative stress as well as $\mathrm{A} \beta$ - (and tau-) induced neurodegeneration has an important role in $\mathrm{AD}$ pathology, as reviewed by the neuroinflammation working group. ${ }^{18}$

Table 1. Characteristics of Early-Onset AD. ${ }^{2 \mathrm{c}}$

\begin{tabular}{|c|c|c|}
\hline Gene & $\begin{array}{l}\text { Age of onset, } \\
\text { years }\end{array}$ & A $\beta$ phenotype \\
\hline APP trisomy 21 & $50 \mathrm{~s}$ & Total $A \beta$ production increased \\
\hline APP mutations & $50 \mathrm{~s}$ & $\begin{array}{l}\text { Total } A \beta \text { production increased } \\
A \beta 42 / A \beta 40 \text { ratio increased }\end{array}$ \\
\hline $\begin{array}{l}\text { APP triplication } \\
\text { of APP gene }\end{array}$ & $50 \mathrm{~s}$ & Total $A \beta$ production increased \\
\hline Presenilin 1 & $40 \mathrm{~s}$ and $50 \mathrm{~s}$ & $\mathrm{~A} \beta 42 / \mathrm{A} \beta 40$ ratio increased \\
\hline Presenilin 2 & $50 \mathrm{~s}$ & $\mathrm{~A} \beta 42 / \mathrm{A} \beta 40$ ratio increased \\
\hline
\end{tabular}

Diagnosis of AD is usually through cognitive testing methods detailed elsewhere, ${ }^{2 \mathrm{c}}$ supported by scanning techniques such as magnetic resonance imaging (MRI).

However, research into biomarkers is a very active and promising field (section 2.4). Prior to development of $\mathrm{AD}$, patients may suffer mild cognitive impairment (MCI), and around 40-60\% of patients with this condition develop AD within five years. ${ }^{19}$ 
Factors that can be used to track the risk of progression of $\mathrm{AD}$ have recently been reviewed, leading to guidelines for the preclinical assessment of the condition. ${ }^{20}$ The pathogenic process leading to $\mathrm{AD}$ may start many years (a period of approximately one decade has been identified ${ }^{20}$ ) before obvious symptoms are noted. The development of biomarkers at an early stage of disease progression would be extremely beneficial.

The insulin/insulin growth factor (IGF) signalling pathway influences ageing and AD progression, as discussed further in section 2.1. Genes undergoing age-related changes in expression have been identified, as have markers of mitochondrial dysfunction in response to oxidative stress. ${ }^{21}$ Another vital regulator of the aging process is autophagy (degradation of intracellular components through lysosomes). Increased autophagy extends lifespan due to reduced insulin-like signalling and it may be stimulated by calorie restriction. Reduced autophagy leads to neurodegeneration, accompanied by the accumulation of ubiquitinylated protein aggregates. ${ }^{21}$ This can occur during normal ageing, but reaches pathological levels in neurodegenerative disorders such as AD. Proteasome dysfunction leads to increased levels of ubiquitinylated protein and to memory deficits in transgenic mice. ${ }^{22}$

The pathology of AD comprises neuritic amyloid plaques and neurofibrillary tangles in the hippocampus, amygdala and association neocortex. Diagnosis with $100 \%$ accuracy can only be achieved post mortem, however diagnosis with $95 \%$ accuracy is possible in living patients using a combination of tools including cognitive testing, brain imaging and analysis of family health history. ${ }^{12 a}$ 
The $\mathrm{A} \beta$ hypothesis (Fig.1) implicates $\mathrm{A} \beta$ as a key causative agent of $\mathrm{AD}$.

Controversies surrounding the $\mathrm{A} \beta$ hypothesis, including the apparently paradoxical presence of $A \beta$ deposits in the brains of people not suffering from dementia, and the cause/effect nature of $A \beta$ deposition, have been discussed. ${ }^{3,6 e, 23}$ However, these deposits are diffuse and have none of the characteristic surrounding neuritic and glial cytopathology found in mature neuritic plaques. ${ }^{6 e, 24}$ Biochemical assays such as ELISA and Western blotting indicate that levels of soluble $A \beta$ correlate better with the presence and extent of cognitive defects than simple plaque counts. $^{25}$

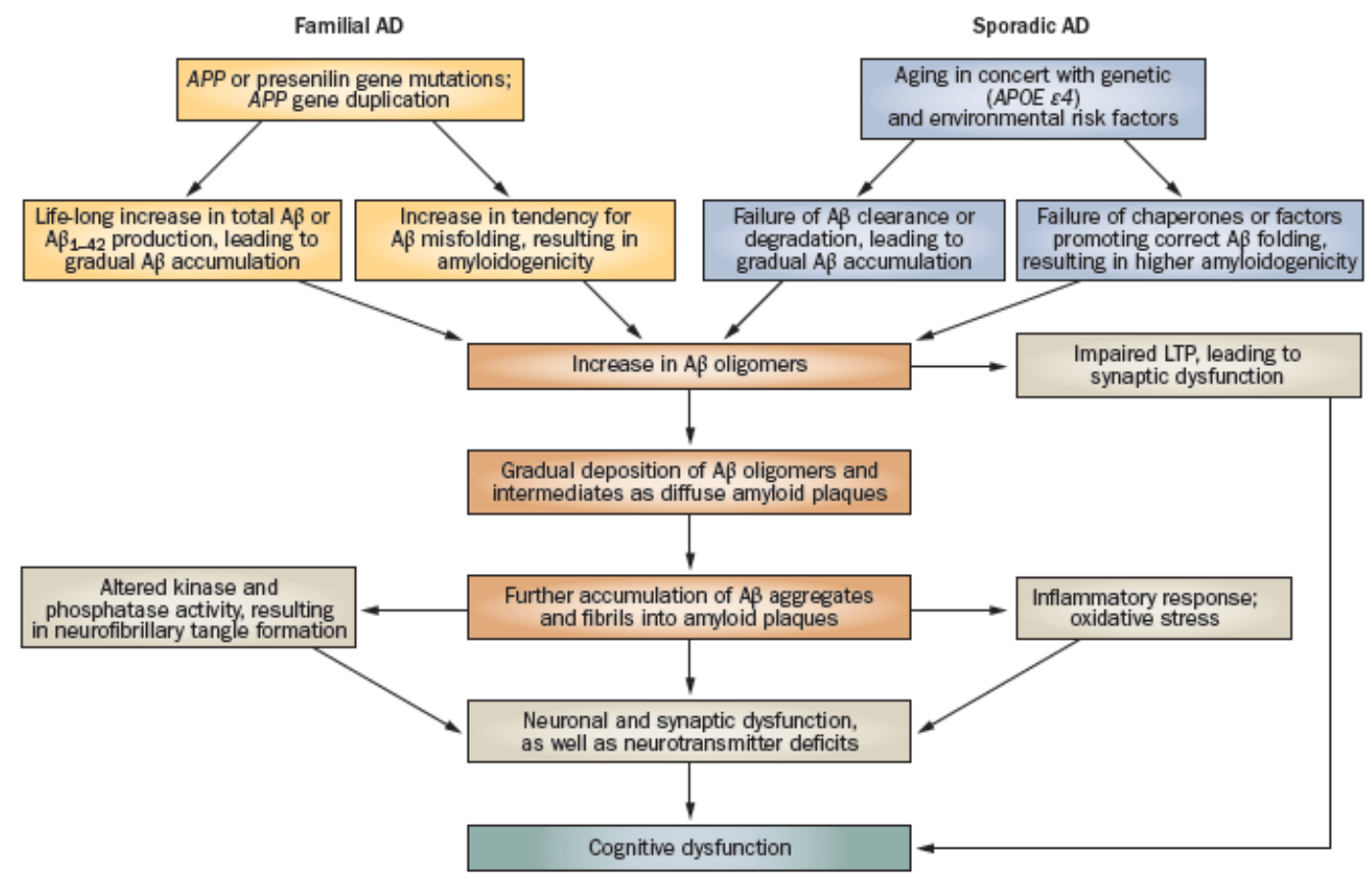

Fig.1. Amyloid cascade hypothesis of AD. ${ }^{26}$ Reprinted by permission from Macmillan Publishers Ltd: Nature Reviews Neurology 6, 131, copyright 2010. 
The term amyloid refers to protein deposits resembling those first observed for starch (amyloid originally meaning starch-like). It is now specifically associated with proteins and peptides adopting fibrils based on the cross- $\beta$ structure in which the peptide backbone is orthogonal to the fibril axis. ${ }^{27}$ The $\beta$-sheets form fibrils, which have an internal structure such as parallel protofilaments, and the fibrils themselves can further aggregate into larger fibres or bundles (which often comprise twisted fibrils). ${ }^{6 b, 27 d}$ Figure 2 shows representative fibril morphologies for $A \beta$ peptides, the fibril morphology depends on preparation conditions, and fibril polymorphism is also observed and examples of other fibril structures are shown in section 4.1.

(a)

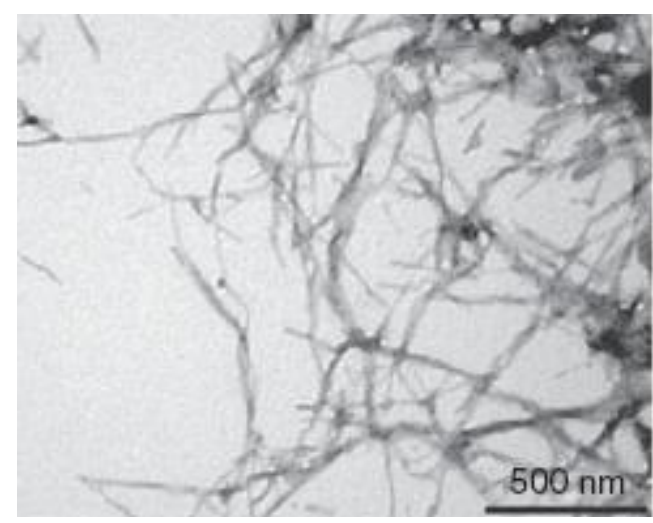

(b)

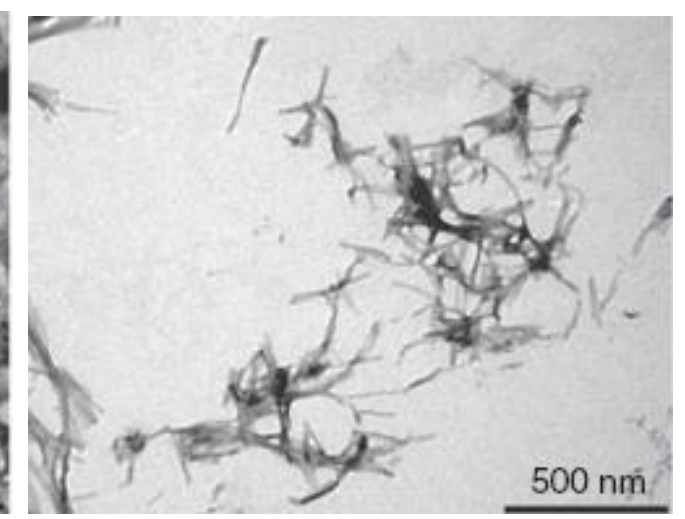

Fig.2. "Typical” A $\beta$ fibril morphology by TEM, obtained from incubated $50 \mu \mathrm{M}$ solutions of (a) $\mathrm{A} \beta 40$, (b) $\mathrm{A} \beta 42 .^{28}$

Figure 3 shows a timeline of some of the principal discoveries in AD research, ${ }^{3,29}$ also the subject of other historical overviews. ${ }^{2 a, 6 e, 30} \mathrm{AD}$ is named after Alois Alzheimer who first described the condition now named after him in $1906{ }^{2 a, 31}$ It is generally acknowledged that the first paper to identify $A \beta$ in association with neuropathology was by Glenner and Wong in $1984,{ }^{32}$ who identified a $4 \mathrm{kDa}$ major 
component of $A \beta$ extracted from the blood vessels of a patient with Down's syndrome. By middle age, the brains of Down's syndrome patients inevitably display the neuropathological features of $\mathrm{AD}$, i.e. deposition of $\mathrm{A} \beta$ plaques and $\mathrm{AD}$-type brain lesions, although mental retardation from birth is due to other causes. Glenner and Wong were also able to sequence the first 28 amino acids of $A \beta$. In the late 1980 's, several different groups were able to use Glenner and Wong's A $\beta$ sequence to clone the gene encoding amyloid precursor protein (APP) and map it to chromosome 21. ${ }^{10,33}$ Chromosome 21 is duplicated in Down's syndrome, hence the correlation with $\mathrm{A} \beta$ deposition which occurs early in this condition. 


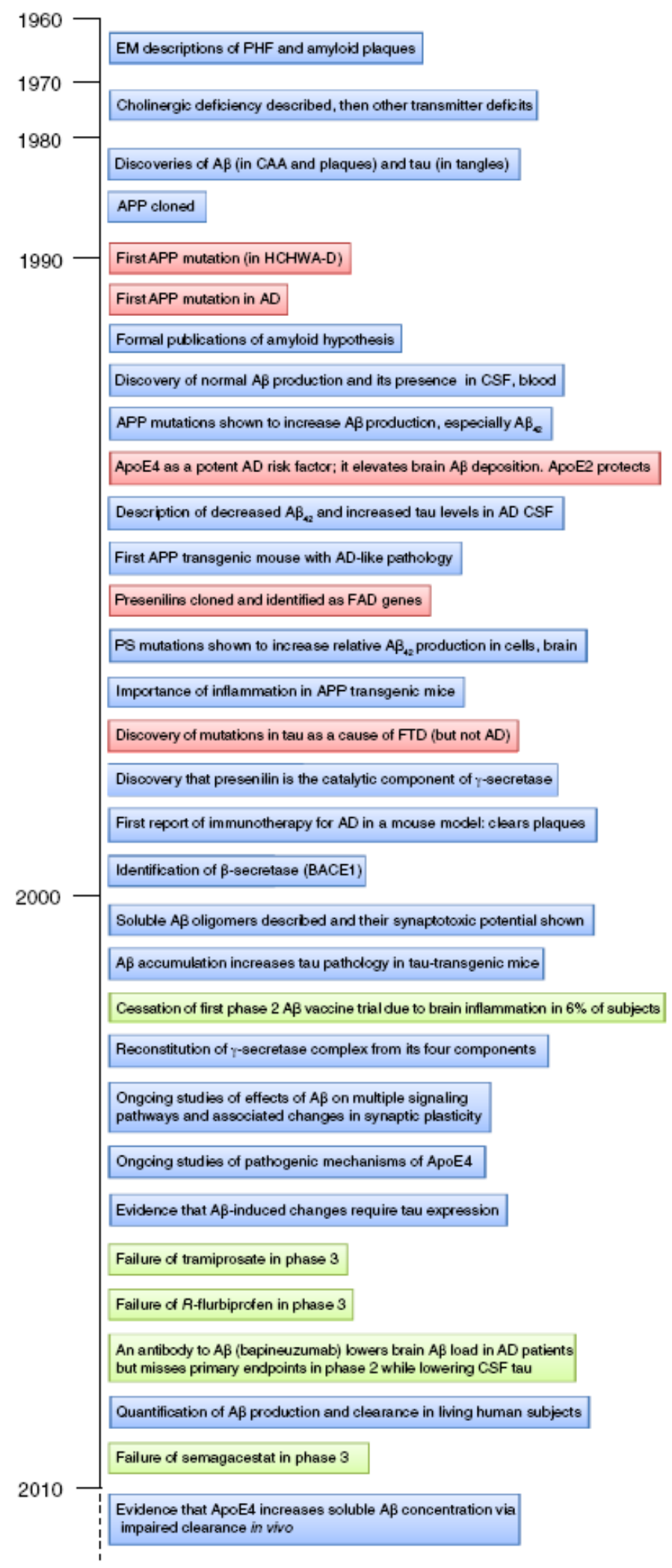

Fig.3. Timeline of selected AD discoveries including those associated with the $\mathrm{A} \beta$ hypothesis. ${ }^{3}$ Reprinted by permission from Macmillan Publishers Ltd: Nature

Medicine 17, 1060, copyright 2011. 
There are two principal variants of the amyloid peptide in humans - A $\beta 40$ and $A \beta 42$ (this notation will be used consistently for the whole peptide with the number of residues indicated, for fragments the sequence will be indicated). ${ }^{\text {1a }}$ The former is more abundant, however $A \beta 42$ forms fibrils more rapidly. ${ }^{1 \mathrm{a}, 6 \mathrm{~d}, 34} \mathrm{~A} \beta 43$ is also observed $^{35}$ as are peptides truncated at the $\mathrm{C}$ terminus ${ }^{36}$ such as $\mathrm{A} \beta 39 .{ }^{37} \mathrm{~N}$-terminal truncated peptides are also detected. ${ }^{36,38}$ Tryptic degradation of $\mathrm{A} \beta$ from $\mathrm{AD}$ patient brains also revealed heterogeneous fragments from the $A \beta(1-5)$ and $A \beta(6-16)$ domains. $^{36}$

It is now thought that oligomers formed in the initial self-assembly process are the toxic agents. ${ }^{2 \mathrm{a}, 2 \mathrm{~d}, 39}$ This is discussed in more detail in Section 2.6. Oligomerization of $\mathrm{A} \beta$ occurs intracellularly, as revealed by in vivo experiments on human cerebrospinal fluid which yielded SDS-stable dimers of $A \beta .^{40}$ Incubation did not lead to the production of extracellular oligomers. However, oligomers were detected in neural and non-neural cell lines. The importance of intracellular A $\beta$ production and its relationship to extracellular production, and re-uptake has been discussed. ${ }^{41}$ Whether intra- and inter- cellular pools of $A \beta$ are distinct or related has been the subject of studies with contradictory conclusions. However, it does appear that extracellular A $\beta$ may originate from intraneuronal sources, and a dynamic equilibrium may exist between these pools. ${ }^{41}$ Since A $\beta$ is produced via cleavage of APP in membranes (Section 2.2.3) its sites of production include the plasma membrane, but also within the cell in the Golgi and endoplasmic reticulum, as well as endosomes and lysozomes. $^{41}$ 
There is a relationship between the incidence of $\mathrm{AD}$ and cerebral ischemia (reduction in blood supply), eg. following a stroke or other cerebrovascular or cardiovascular condition. ${ }^{42}$ Oxidative stress, eg. hypoxia (oxygen deprivation) or ischemia may cause an increase in A $\beta$ levels in the brain due to an upregulation of APP processing. ${ }^{43} \mathrm{~A}$ correlation between serial brain interstitial fluid (ISF) concentration and neurological status (after acute injury) has been noted, $A \beta$ concentration increasing with improved neurological status. $^{44}$

Amyloid $\beta$ is produced by proteolytic cleavage of APP, a transmembrane protein discussed further in Section 2.2.3. ${ }^{2 \mathrm{a}}$ The peptide $\mathrm{N}$ terminus is created by cleavage by $\beta$-secretase in the extracellular domain of APP, and the C-terminus results from intramembrane cleavage by $\gamma$-secretase. A third enzyme, $\alpha$-secretase cleaves between amino acids 16 and 17 in $A \beta$, thus hindering fibrillization. The cleavage by $\gamma$ secretase is presenilin-dependent. ${ }^{45}$ As discussed further in Section 2.2.3, $\gamma$-secretase is a protein complex involving presenilins, nicastrin, APH-1 and PEN-2, all of which are required for $\gamma$-secretase function. ${ }^{46}$ Selkoe thoroughly reviews the historical literature concerning the relationship between the presenilins and $\gamma$-secretase. ${ }^{\text {1a }}$

Whilst there have been numerous reviews on the amyloid hypothesis of $\mathrm{AD}$, there are few up-to-date reviews that also discuss the biophysical aspects of $A \beta$ self-assembly and its influence on AD. The present review aims to provide a unified view of the biological, neurochemical and biophysical aspects of $A \beta$ aggregation and its relationship to $\mathrm{AD}$. In addition, this review provides a current overview of developments in potential therapeutic strategies. 
This review is organized as follows. The first section concerns the features of AD and the properties of $\mathrm{A} \beta$ and APP in vivo, including biological and neurochemical characteristics. The development of biomarkers is also considered. This is followed by discussion of therapeutic compounds. The final sections are focussed on different aspects of the biophysical properties of $A \beta$.

\section{BIOLOGICAL, BIOCHEMICAL AND NEUROCHEMICAL}

\section{CHARACTERISTICS OF AD, INVOLVING A $\beta$ AND APP}

\subsection{Genetic Markers for AD}

\subsubsection{Genetic Risk Factors}

Several genes have been linked to late-onset Alzheimer's disease, most importantly the gene encoding apolipoprotein $\mathrm{E}(A P O E)^{47}$, especially the $\varepsilon 4$ allele. ${ }^{47,47 \mathrm{~b}, 47 \mathrm{~d}, 48}$ This is believed to cause the aggressive form of AD with earlier onset. ${ }^{3,49}$ APOE (and related APOC1) diagnostic testing systems have even reached the market although some products have been withdrawn due to IP issues. ${ }^{50}$ The compound rosiglitazone may ameliorate neuronal dendritic spine loss caused by ApoE- $\varepsilon 4$, and thus improve cognition in $\mathrm{AD}$ patients. ${ }^{51}$

Mutations in the genes for APP,$^{52}$ presenilin 1 and 2 (PSEN1 or PS1 and PSEN2 or $P S 2)^{1 \mathrm{a}, 53}$ have a role in hereditary forms of AD. ${ }^{54}$ Dominantly inherited forms represent only $1-3 \%$ of the total number of cases of $\mathrm{AD}$, most of which are sporadic. $^{2 \mathrm{a}, 11,54}$ Mutations in PS1 and PS2 potentially account for a large fraction of early-onset cases of familial AD. ${ }^{1 \mathrm{a}, 55}$ Mutations in the presenilins cause an increase in $\mathrm{A} \beta 42$ in $\mathrm{AD}$ patients ${ }^{1 \mathrm{~b}, 55 \mathrm{a}, 56}$ and also transfected cell lines and transgenic animals 
expressing mutant forms of PS1 or PS2. ${ }^{57}$ and this occurs selectively for A $\beta 42 / 43$

over $A \beta 40 .{ }^{56-57}$ Certain presenilin mutations can disrupt the leakage of calcium (see the discussion of the calcium channel hypothesis in section 2.7) induced by presenilins from the endoplasmic reticulum leading to supranormal release and dyshomeostatis. ${ }^{58}$ This has been investigated in electrophysiology experiments. ${ }^{55,58-59}$ Missense mutations in PS1 are associated with early and aggressive forms of AD, ${ }^{\text {a }}$ A 342 plaques being observed as early as 3-4 months. ${ }^{60}$ The G209V, A260V and E280A presenilin A mutations lead to substantial overexpression of $A \beta 42$ in the brains of FAD patients. ${ }^{61}$ Mutations in PSENI are also associated with acne inversa although a correlation between this condition and AD has not been noted. ${ }^{62}$

The gene encoding ApoE was the first confirmed susceptibility locus for sporadic late onset $\mathrm{AD}$, and its alleles have been widely studied. A recent study using microdialysis in a PDAPP/TRE mouse model (to be discussed shortly) indicates that different isoforms of the gene differentially regulate $\mathrm{A} \beta$ clearance from the brain. ${ }^{63}$ A genomewide association study identified three other loci, within $C L U$ (which codes for apolipoprotein J, ApoJ or clusterin), ${ }^{54,64}$ within $C R 1$ (complement receptor 1$)^{54}$ or within PICALM. ${ }^{64}$ Single nucleotide polymorphs (SNPs) at these loci (as well as $A P O E$ ) were associated with AD risk. ApoE and CLU are the most abundantly expressed apolipoproteins in the central nervous system. ${ }^{54}$ Earlier work had shown that ApoJ is over-expressed in individuals with $\mathrm{AD}$ and is present in $\mathrm{CSF}^{65}$ and amyloid plaques. ${ }^{66}$ Clusterin binds soluble $A \beta$ to form complexes (especially with the more toxic $A \beta 42$ peptide) which can cross the blood-brain barrier ${ }^{67}$ It promotes amyloid plaque formation and is critical for toxicity towards neurons. ${ }^{68}$ Bell et al . performed studies on the clearance of radiolabelled $\left({ }^{125} \mathrm{I}\right) \mathrm{A} \beta$ using a mouse model 
and found that $A \beta 42$ is cleared more slowly than $A \beta 40$ and that $A \beta, A p o E$ and $A p o J$ (detected using human-specific ELISAs) are cleared from the brain by different transport mechanisms. ${ }^{67 \mathrm{~b}}$

A study using a yeast model has identified genetic factors influencing A $\beta$ toxicity, including PICALM but also other previously unidentified genes associated with protein trafficking, stress and metabolism. ${ }^{69}$ This work also confirmed the effect of PICALM on A $\beta$ toxicity using a $C$. elegans model and also using rat cortical neurons. ${ }^{69}$ In terms of mechanisms, the authors suggested that $A \beta$ affects the endocytic trafficking of a plasma membrane receptor. Another genome-wide survey revealed an association between late-onset $\mathrm{AD}$ in carriers of the $A P O E-\varepsilon 4$ allele and SNPs from the $G R B$-associated binding protein $2(G A B 2)$ gene. ${ }^{70}$ ApoD has also been associated with $\mathrm{AD}$, this lipoprotein circulates as a components of serum high density lipoproteins (HDL) and may be involved in cholesterol transport. ${ }^{71}$ Apolipoprotein D is involved in lifespan extension in Drosophila, conferring resistance to oxidative stress, and its expression is induced in the AD brain. ${ }^{71}$

Another gene that was identified as a risk for AD is CALHMI (denoting calcium homeostasis modulator 1). ${ }^{72}$ The CALHM1 protein is localized in the cell membrane, and increased expression leads to enhanced calcium levels within the cytoplasm. Single nucleotide polymorphism of the gene leads to changes in AD susceptibility, specifically a P86L substitution leads to increased A $\beta$ levels. However, these findings have been challenged - Bertram et al. also examined several family-based datasets and number of prior genome-wide association study (GWAS) datasets ${ }^{70,73}$ and found 
no correlation between $C A L H M 1$ and $\mathrm{AD} .{ }^{74}$ However, the authors of the original study dispute this analysis. ${ }^{75}$

The role of the Orphan G protein-coupled receptor (GPR3) as a modulator of A $\beta$ production has recently been identified. ${ }^{76}$ The GPR3 gene has been mapped to a candidate $\mathrm{AD}$ linkage region in one chromosome as part of a large-scale genome screen using an NIMH (National Institute for Mental Health) sample. ${ }^{77}$ GPR3 expression leads to an increase in production of the $\gamma$-secretase complex, and its cell surface localization, in the absence of an effect on Notch processing (discussed further in Section 3.2.1). ${ }^{76}$ Notch proteins are transmembrane proteins involved in development and signaling pathways and a key challenge in the development of effective $\gamma$-secretase inhibitors is to avoid side effects caused by interference with these pathways. GPR3 was found to be highly expressed in areas of the normal brain implicated in $\mathrm{AD}$ and is elevated in the sporadic $\mathrm{AD}$ brain. It thus represents a potential target for therapeutic treatment.

Other proteins associated with A $\beta$ production or APP processing include the serotonin receptors ${ }^{78}$ and the prostaglandin $\mathrm{EP} 2$ receptor. ${ }^{79}$ Prostaglandin $\mathrm{E}_{2}$ is produced during inflammation due to activity by cytosolic phospholipase $\mathrm{A}_{2}$ (PLA2) or cyclooxygenase 2 (COX-2). ${ }^{79}$ The latter enzyme is upregulated in AD brain frontal cortex and synthetic $\mathrm{A} \beta$ peptides induce $\mathrm{COX}-2$ expression in SH-SY5Y neuroblastoma cells in vitro. ${ }^{80} \mathrm{COX}-2$ is involved in the inflammatory response and is the target of NSAIDS (non-steroidal anti-inflammatory drugs). A population-based study pointed to the elevation of serum levels of pregnancy zone protein $(\mathrm{PZP})$ in presymptomatic $\mathrm{AD}$, compared to controls. ${ }^{81}$ 
Genome-wide studies of genes associated with aging indicate that the insulin/IGF-1 signalling pathway may be involved in Alzheimer's. Reduced signalling causes decreased AD pathology in mice ${ }^{82}$ while paradoxically increased signalling may also be neuroprotective. ${ }^{21}$

\subsubsection{Transgenic Mice}

Many studies use the PDAPP mouse, also known as PDGF-hAPP (from plateletderived growth factor) mouse, which overexpresses mutant human APP (V717F mutant) under control of mouse regulatory elements and leads to $A \beta$ plaque deposition. ${ }^{15,83}$ The PDAPP/TRE model expresses human ApoE. The TgCRND8 murine model of AD expresses a doubly mutant (K670N/M671L and V717F) human APP695 transgene. ${ }^{84}$ Tg2576 APP mice expressing the Swedish FAD variant of human $\mathrm{APP}_{695}$ (section 2.2.3) ${ }^{85}$ which leads to a selective increase in $\mathrm{A} \beta 42 / 43$ production have also been used in $\mathrm{A} \beta$ immunization experiments. ${ }^{86} \mathrm{Tg} 2576$ mice develop memory deficits due to the extracellular accumulation of specific $A \beta$ oligomeric species, i.e. dodecamers. ${ }^{87}$ A doubly mutant transgenic mouse including the APP(Swe) and mutant PS1 (M146L) has been developed and exhibited a large selective enhancement of $A \beta 42$ and plaque deposition. ${ }^{60}$ The $3 x \mathrm{Tg}$ model in a triply transgenic mouse contains PS1(M146V), APP(Swe), and tau(P301L) transgenes ${ }^{88}$ and this has been used to investigate the interplay of $A \beta$ and tau (neurofibrillary tangles) pathologies (section 2.8). Contrary to doubly transgenic mice lacking the APP transgene, deposition of plaques and synaptic dysfunction (LTP deficits) are observed with the $3 \times$ Tg model. ${ }^{88}$ The APP23 mouse overproduces A $\beta 40$ and the APPPS 1 mouse overexpresses $A \beta 42 .^{89}$ 


\section{$2.2 \mathrm{~A} \beta$ in vivo}

\subsubsection{Characteristics of $A \beta$ in vivo}

Evidence that the $A \beta 42$ form of $A \beta$ is the variant preferentially implicated in $A D$ comes from several sources. Studies of the kinetics of aggregation (through turbidity measurements) indicate that $\mathrm{A} \beta 42$ nucleates more rapidly and is more fibrillogenic than $A \beta 40 .{ }^{34,90}$ The toxicity of $A \beta 42$ is much greater than $A \beta 40^{91}$ due to its greater tendency to fibrillise. Some mutations in APP in cultured cells (discussed in section 2.2.2) lead to increased levels of A $\beta 42$, whereas wildtype APP predominantly releases $A \beta 40 .^{92}$ It is also found that $A \beta 42$ is the principal component of diffuse $A \beta$ plaques and plaques generated from APP mutants ${ }^{93}$ and in homogenized brain tissue, ${ }^{94}$ and that early and selective deposition of $A \beta 42$ is observed in the brains of AD patients (shorter peptides with different $\mathrm{N}$ termini are also found). ${ }^{36,38}$ Despite its lower toxicity, $A \beta 40$ is actually produced by a factor of ten times more than $A \beta 42$, by $\gamma$-secretase cleavage. ${ }^{41}$

Whilst $A \beta$ is generally associated with disease, a functional role for the peptide has also been suggested. Tanzi and coworkers have demonstrated that it is an antimicrobial peptide, i.e. that it is involved in immune reactions. ${ }^{95}$ Antimicrobial activity of $\mathrm{A} \beta 40$ and $\mathrm{A} \beta 42$ has been demonstrated against eight common microorganisms including E. coli and $S$. aureus. This activity can be blocked by immunodepletion of AD brain homogenates with anti-A $\beta$ antibodies. ${ }^{96}$ Temporal lobe tissue from $\mathrm{AD}$ patients showed higher antimicrobial activity than material from the 
brains of age-matched non-AD subjects. Balin's group have suggested that A $\beta$ may be part of the control mechanism following infection by $C$. pneumoniae,${ }^{97}$ and that it can mediate infection of cells with this bacterium. ${ }^{98}$ Smith and coworkers argue that $\mathrm{A} \beta$ production is a host response to an underlying condition that develops with age. ${ }^{99}$ However, this is becoming an increasingly contrarian viewpoint in view of the mass of data implicating $A \beta$ as the causative agent. The $\varepsilon 4$ allele of the $A P O E$ gene, a marker for EOAD (section 2.1.1) may have a beneficial role in enhanced cognitive skills. $^{100}$

The fraction of different variants of $A \beta$ has been investigated. Based on analysis of cell lysates and also tissue from mouse brain, $A \beta 40$ has been found to constitute approximately $90 \%$ of the secreted $\mathrm{A} \beta$ and $\mathrm{A} \beta 42$ comprises $\sim 10 \%,{ }^{57 \mathrm{~b}-\mathrm{d}}$ although somewhat lower ${ }^{57 c}$ and much higher ${ }^{57 \mathrm{e}}$ fractions have also been reported. $\mathrm{A} \beta 42$ is the most commonly found variant in human CSF.

Since APP is expressed in most peripheral cells, A $\beta$ is present in plasma in addition to CSF. The level of A $\beta 40$ in plasma is generally under $200 \mathrm{pM}$, and of $A \beta 42(3)$ is under $60 \mathrm{pM}$, although both are elevated in patients with PS1 or PS2 mutations or in patients with presymptomatic or symptomatic APP patients. ${ }^{56}$ The physiological concentration of $\mathrm{A} \beta$ (in $\mathrm{AD}$ patients) in human CSF has been reported by several groups. An $A \beta$ concentration of less than $500 \mathrm{pg} / \mathrm{ml}(0.1 \mathrm{nM})$ is indicative that $A \beta$ is accumulating in the brain and not circulating in the CSF. Using an ELISA assay, Mehta et al. reported for $\mathrm{A} \beta 40 c=30 \mathrm{nM} .^{101}$ and for $\mathrm{A} \beta 42 c=8 \mathrm{pM} \cdot{ }^{101}$ On the other hand, Ida et al. using a Western blot assay reported a lower $c=6 \mathrm{nM}$ for $\mathrm{A} \beta 40$ but a 
much higher $c=60 \mathrm{pM}$ for $\mathrm{A} \beta(1-42) .{ }^{37}$ Similar values for $\mathrm{A} \beta 42$ for AD patients are reported by Motter et al. ${ }^{102}$ Values in plasma are also available. ${ }^{56,101}$ The CSF concentration of $\mathrm{A} \beta 40$ for $\mathrm{AD}$ patients is not significantly different to that for nondemented control patients, however the concentration of $A \beta 42$ is lower for $A D$ patients. ${ }^{37,101-102}$ This is another evidence for $A \beta 42$ as the disease-related species. Peripheral adminstration of monoclonal antibodies leads to a rapid increase in plasma $\mathrm{A} \beta .^{103}$ The physiological concentration of $\mathrm{A} \beta$ (variant not defined) in normal human CSF is around 1- $2 \mathrm{nM}$ according to ref. ${ }^{104}$ whereas a value of $3-8 \mathrm{nM}$ is cited elsewhere. ${ }^{1 \mathrm{a}, 102}$ According to Podlisny et al. the physiological concentration of A $\beta 40$ is $0.25-2.5 \mathrm{nM},{ }^{105}$ in agreement with the value $c=0.6 \mathrm{nM}$ reported by other groups. ${ }^{106}$ There is no correlation between plasma $A \beta 40$ and $A \beta 40$ load (in the range $0-40 \%$ for $46 \mathrm{nM} \mathrm{A} \beta 40$ in PDAPP mouse plasma) in the absence of anti-A $\beta$ antibodies. ${ }^{103}$ The concentration of $A \beta$ in serial brain interstitial fluid has also been reported. ${ }^{44}$ Ida et al. also detected the presence of $\mathrm{N}$-terminally truncated $\mathrm{A} \beta$ species in CSF and plasma. ${ }^{37}$

The production of $A \beta 40$ and $A \beta 42$ in the human central nervous system (CNS) does not appear to be different for AD patients compared to control, however the rate of clearance is significantly reduced for $\mathrm{AD}$ patients. ${ }^{107}$ Production of $\mathrm{A} \beta$ is discussed in the following section. Low-density lipoprotein receptor-related protein (LRP) and the receptor for advanced glycation end products (RAGE) are involved in receptormediated flux of $\mathrm{A} \beta$ across the $\mathrm{BBB}$ as part of the clearance mechanism. ${ }^{108}$ Clearance of $A \beta$ from the brain to the periphery appears to be mediated by LRP while RAGE is implicated in A $\beta$ efflux back into the CNS (Fig.4). ${ }^{108 b}$ 


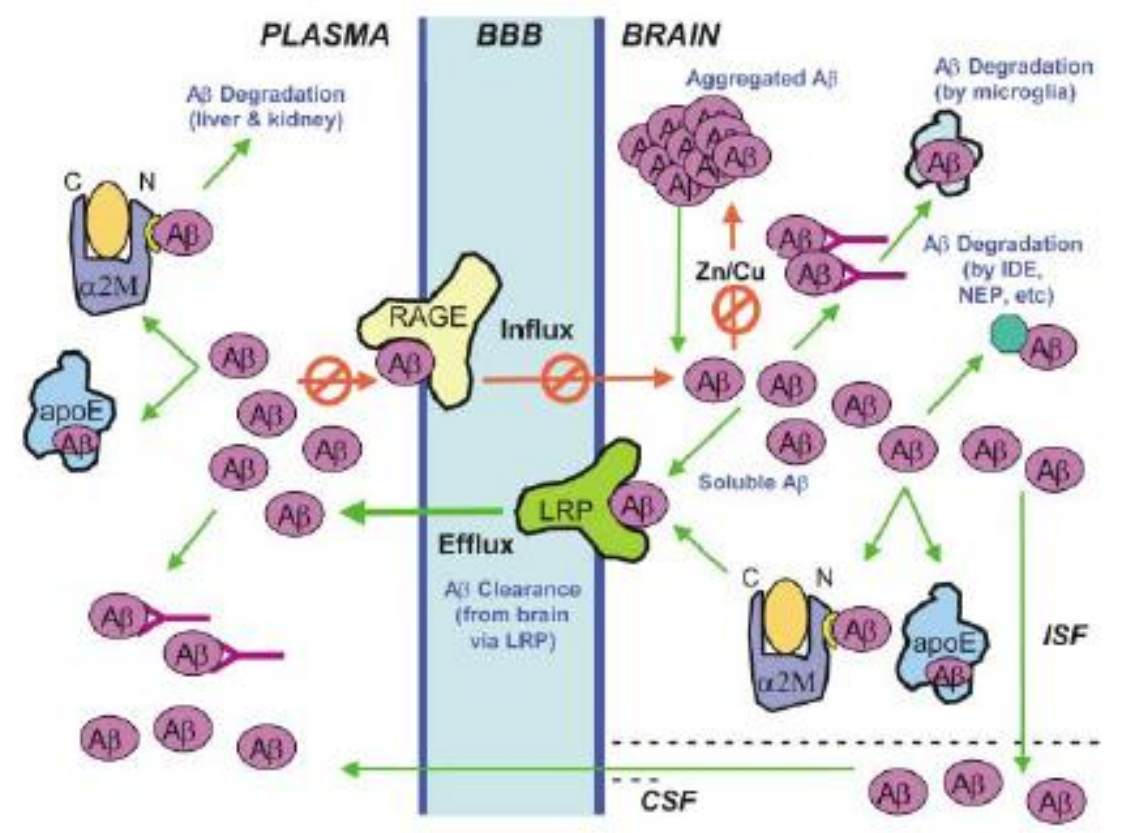

Fig.4. Clearance mechanisms of $A \beta$ involving LRP and RAGE. ${ }^{108 b}$ Blocking interaction of $A \beta$ with zinc and copper may clear $A \beta$. Soluble $A \beta$ can be removed via enzymatic degradation (via peptidases such as insulin degrading enzyme IDE or neprilysin NEP, and subsequent degradation by activated microglia) or receptormediated clearance. LRP receptor-mediated clearance can occur by direct binding or initial binding to the LRP ligands/A $\beta$ chaperones ApoE and $\alpha 2 \mathrm{M}$ which can deliver $\mathrm{A} \beta$ to peripheral sites of degradation (liver or kidney). These chaperones can also deliver $A \beta$ across the BBB into the brain. This process can also occur via the RAGE receptor. Green arrows show pathways that might be pharmacologically relevant. Reprinted from Tanzi, R. E. et al., Neuron 2004, 43, 605, Copyright 2004, with permission from Elsevier

\subsubsection{Modelling $A D$ and $A \beta$ Deposition in Other Organisms}


AD can be modeled using a variety of organisms including fruit flies (Drosophila melanogaster), ${ }^{109}$ nematode worms (Caenorhabditis elegans), ${ }^{69,110}$ potatoes, ${ }^{111}$ and yeast (Saccharomyces cerevisae). ${ }^{69,112}$ C. elegans has attracted particular attention as a model to study the function of presenilins, especially in relation to Notch (Section 3.3) since there are great similarities between the sel-12 gene of C. elegans and presenilin genes. ${ }^{1 \mathrm{a}, 113}$ Drosophila melanogaster has been engineered to express both wild type human and arctic mutant $\mathrm{A} \beta 42 .{ }^{109 \mathrm{~d}}$ Yeast exhibits $\alpha$-secretase activity on APP. ${ }^{112}$ The later study showed that yeast can be used to model links between A $\beta$, endocytosis and human $\mathrm{AD}$ risk factors. ${ }^{69} \mathrm{~A} \beta(\mathrm{M} 1-40)$ and $\mathrm{A} \beta(\mathrm{M} 1-42)$ i.e. with an $\mathrm{N}$ terminal methionine substitution, can be expressed in E. coli. ${ }^{28,114}$ The fibrils formed by the recombinant peptides are indistinguishable than those from chemically synthesized peptides.

\subsubsection{APP and the Production of $A \beta$}

APP belongs to the family of type 1 transmembrane glycoproteins. ${ }^{115}$ It has been hypothesized to act as a vesicular receptor for the motor protein kinesin-I. ${ }^{109 \mathrm{~b}}$ The production of $A \beta$ in the amyloidogenic pathway involves the sequential cleavage of APP by $\beta$-secretase and $\gamma$-secretase (Fig.5). The enzyme $\beta$-secretase is an integral membrane aspartyl protease encoded by the $\beta$-site APP-cleaving enzyme 1 gene $(B A C E 1)^{1 \mathrm{a}, 1 \mathrm{~b}, 116}$ while $\gamma$-secretase is a membrane-bound protease complex consisting of at least four components including the presenilins (PS1 and PS), nicastrin, and the genes $A P H-1$ and PEN-2. ${ }^{110 b, 117}$ The $\gamma$-secretase complex may also function as an aspartyl protease. ${ }^{1 \mathrm{a}, 117 \mathrm{~b}}$ It has been proposed that $\mathrm{A} \beta$ up-regulates its own production by increasing BACE1 expression, ${ }^{118}$ possibly involving oxidative stress. ${ }^{119}$ 


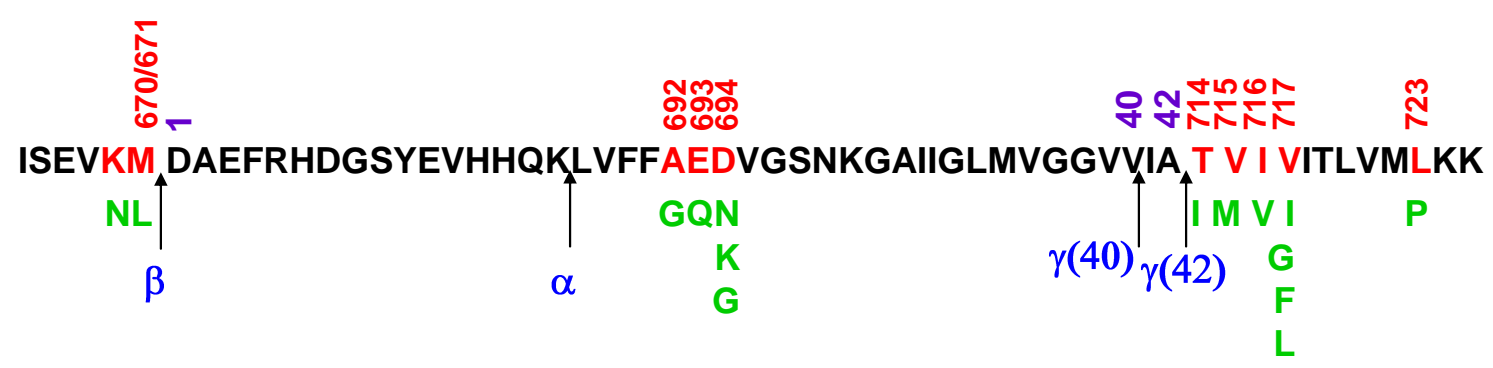

Fig.5. Mutations in APP associated with A $\beta$ production and expression and AD. Redrawn from ref. ${ }^{\text {la }}$ Secretase enzymes are indicated in blue, with cleavage sites arrowed. APP sequence numbers are shown in red, A $\beta$ in purple. Mutants are indicated with green letters.

In the non-amyloidogenic pathway, a third enzyme, $\alpha$-secretase cleaves between amino acids 16 and 17 in $A \beta$, thus hindering fibrillization of the full peptide. The $\alpha$ secretases belong to the "A Disintegrin and Metalloprotease" (ADAMs) family of zinc metalloproteinases. ${ }^{120}$

Recent work suggests that in late onset Alzheimer's disease, $A \beta$ accumulation occurs intracellularly in late endosomes where enzymes $\beta$-secretase and $\gamma$-secretase cleave $\mathrm{A} \beta$, the latter in a presinilin-dependent fashion. ${ }^{121}$ The intracellular sites of $\mathrm{A} \beta$ production occur where APP is located, as shown in Fig.6. ${ }^{41}$ The gene involved in APP recycling in endosomes has been identified, and is termed SORL1 and the associated protein is SORLA or LR11. Normally the protein product of the gene directs APP into recycling endosomes (retromer recycling endosomes, Fig.6), however mutations produce a decrease in protein product which leads to the pathway 
where $A \beta$ production via fragment $C 99$ is increased by enzymes in the late endosomes. ${ }^{121}$ It has been reported that the proteolytic processing of $A \beta$ is regulated by glycogen synthase kinase- 3 isozymes, ${ }^{46}$ however this has been disputed. ${ }^{122}$

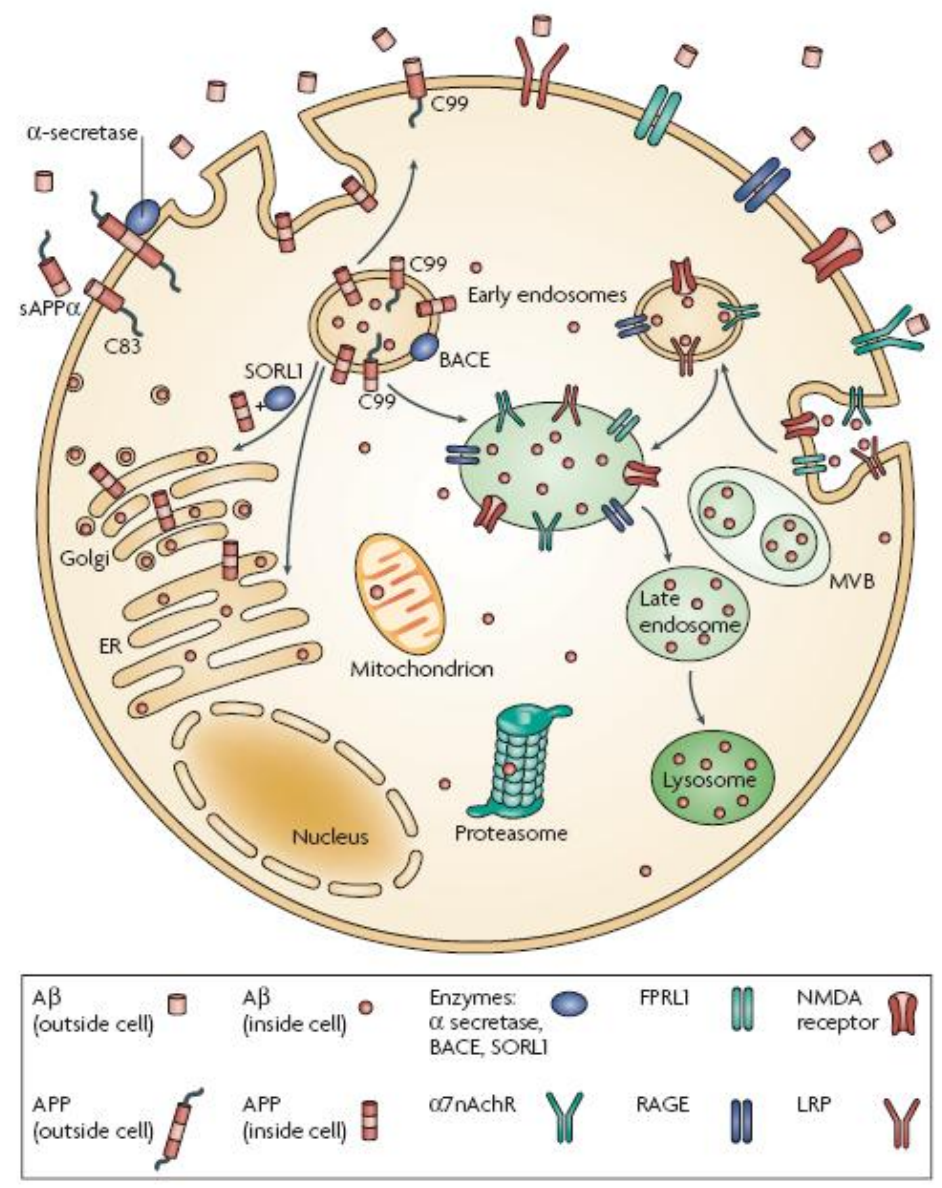

Fig.6. Sites of intracellular $A \beta$ production. ${ }^{41} A \beta$ is produced within the endoplasmic reticulum (ER) and Golgi system and secreted. APP is localized in the plasma membrane, where it is cleaved by $\alpha$-secretase, releasing soluble APPs into the extracellular space and leaving an 83-amino acid fragment known as C83 within the membrane. Unprocessed APP can be internalized into early endosomes. In the presence of SORL1, APP is recycled back to the Golgi in retromer endosomes. Early endosomes contain BACE1 which cleaves APP to produce a 99 residue fragment C99, retained within the membrane. C99 can be shuttled back to the ER to be 
processed into $A \beta$ by $\gamma$-secretase in the $E R$, shuttled back to the plasma membrane where the $\gamma$-secretase complex is also found, or processed to $A \beta$ within the endosome/lysosome system. Extracellular $\mathrm{A} \beta$ (i.e. previously secreted $\mathrm{A} \beta$ ) can bind to cell surface receptors (including RAGE, LRP, FPRL1, NMDA receptors and $\alpha 7$ $\mathrm{nAChR}$ ) and the receptor- $\mathrm{A} \beta$ complex can be internalized into early endosomes [FPRL1 denotes FMLP-receptor-like protein, NMDA denotes $N$-methyl-D-aspartate and $\alpha 7$-nAChR the $\alpha 7$ nicotinic acetylcholine receptor]. Intracellular accumulation of $\mathrm{A} \beta$ mainly occurs in the multivesicular body (MVB) and lysosome, but also in the mitochondria, ER, Golgi and cytosol, where it can influence proteasome function. Reprinted by permission from Macmillan Publishers Ltd: Nature Reviews Neuroscience 8, 499, copyright 2010.

In addition to the known isoforms of $A \beta$, i.e. $A \beta 42, A \beta 40$ and $A \beta 38$, shorter fragments have been identified in CSF. ${ }^{120 \mathrm{~b}}$ This suggested a different APP processing pathway involving concerted cleavage of APP by $\alpha$ - and $\beta$-secretases.

It has recently been proposed that rather than A $\beta$, another APP fragment may be involved in $\mathrm{AD}$ (possibly along with $\mathrm{A} \beta$ ). ${ }^{123}$ The N-APP extracellular N-terminal fragment is adjacent to $A \beta$ and is also cleaved by BACE. It triggers the cell death cascade by binding to a neuronal receptor called DR6 (death receptor 6), which is highly expressed in regions of the human brain most affected by $\mathrm{AD}$, in the presence of caspase $6 .^{123}$ 
Fig.5 summarizes the mutations in APP linked to AD. It is notable that these mutations are located just outside the cleavage sites of $\beta$ - and $\gamma$-secretase beyond the $\mathrm{N}$ - and C-termini of $\mathrm{A} \beta$ respectively, as well as close to the $\alpha$-secretase cleavage site within $A \beta$. Mutations within $A \beta$ are expected to enhance the aggregation properties, and this has been shown for the E693Q mutation (Dutch-type) (Table 2, section 2.2.4). The Flemish $A 692 \mathrm{G}$ mutation leads to a mixture of $\mathrm{A} \beta$ plaque and tangle formation as well as microvascular $\beta$-amyloidosis and cerebral hemorrhage due to cerebral amyloid angiopathy. ${ }^{124}$ This mutation also enhances the proportion of APP cleaved by the $\beta$-secretase homologue BACE-2. ${ }^{1 \mathrm{a}, 125}$

APP comprises a group of ubiquitously expressed polypeptides migrating between 110 and $135 \mathrm{kDa}$ on electrophoretic gels. ${ }^{1 \mathrm{a}, 126}$ The heterogeneity arises since there are three main isoforms of human APP with 695, 751 and 770 residues, and additionally due to post-translational modifications including $N$ - and $O$-glycosylation, phosphorylation and sulfation. APP forms containing 751 and 770 residues are widely expressed both in neuronal and non-neuronal cells throughout the body whereas the 695-residue form is expressed more highly in neurons, and occurs at very low abundance in other cells. ${ }^{1 \mathrm{a}}$ The 751 and 770 isoforms contain a KPI (Kunitz-type Protease Inhibitor) domain (Fig.7) and are thus able to inhibit serine proteases such as trypsin and $\alpha$-chymotrypsin. ${ }^{127}$ 


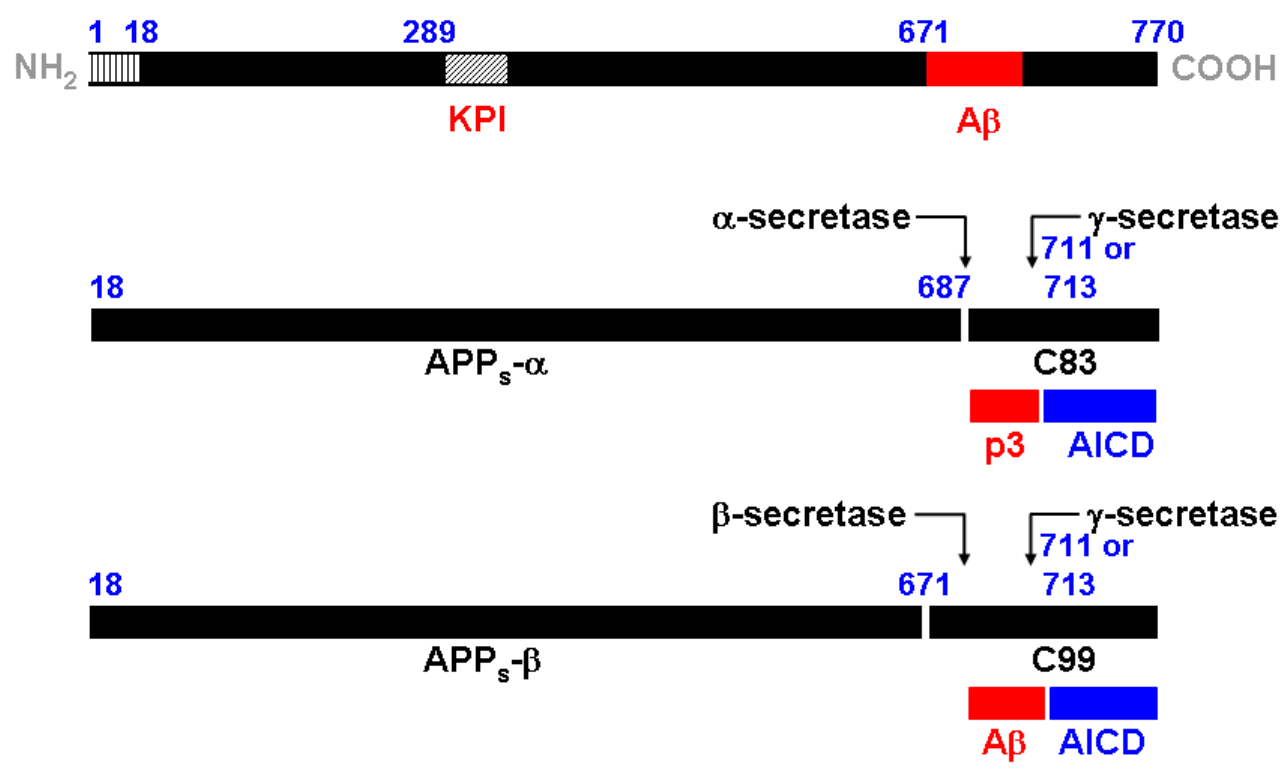

Fig.7. Processing routes of APP to produce A $\beta$ and other peptide fragments (C83, C99 and p3) as well as the soluble $\mathrm{APP}_{\mathrm{s}}$ ectodomain fragments. Redrawn, based on schematics by Selkoe. ${ }^{1 \mathrm{a}, 1 \mathrm{~b}}$ TM denotes transmembrane domain, KPI denotes Kunitztype inhibitor domain which is a spliced exon of 56 amino acids inserted at residue 289. Cleavage of both C83 and C99 C-terminal fragments by $\gamma$-secretase releases the $\beta$-amyloid precursor protein intracellular domain (AICD) into the cytoplasm. A 17residue single peptide is indicated at the $\mathrm{N}$ terminus.

Table 2 presents common mutations in APP. The E693Q mutation was the first to be associated with disease, hereditary cerebral haemorrhage with amyloidosis, Dutch type (HCHWA-D). ${ }^{128}$ The A692G Flemish mutation is associated with cerebral haemorrhage with amyloidosis (CHWA). ${ }^{128}$ A mutation in APP K670M671 $\rightarrow$ N670L671 has been associated with the so-called Swedish FAD. ${ }^{129}$ The London 
variant involves V717I. ${ }^{52,128,130}$ Detailed information on mutations in presenilins is provided elsewhere. $^{10,58 \mathrm{a}}$

Table 2. Effect on A $\beta$ of APP mutations. Adapted from refs. ${ }^{10,131}$

\begin{tabular}{|c|c|c|c|}
\hline Name/FAD variant & Mutation & Effect on APP & Effect on $A \beta$ \\
\hline APP-717 (London) & V717F/G/I & $\begin{array}{l}\text { Differential } \gamma- \\
\text { secretase cut }\end{array}$ & $\begin{array}{l}A \beta 42: A \beta 40 \text { ratio } \\
\text { increased }\end{array}$ \\
\hline $\begin{array}{l}\text { APP-670/671 } \\
\text { (Swedish) }\end{array}$ & $\begin{array}{l}\text { K670N and } \\
\text { M671L }\end{array}$ & $\begin{array}{l}\text { Increased } \beta- \\
\text { secretase cut }\end{array}$ & $\begin{array}{l}\text { Increased } A \beta 40 \\
\text { and } A \beta 42 \text { in plasma }\end{array}$ \\
\hline APP-692 (Flemish) & A692G & $\begin{array}{l}\text { Decreased } \alpha- \\
\text { secretase cut? }\end{array}$ & $\begin{array}{l}\text { Decreased } A \beta 40 \\
\text { and } A \beta 42 \text { in media, } \\
\text { decreased } A \beta \\
\text { aggregation, } \\
A \beta 42: A \beta 40 \text { ratio } \\
\text { increased }\end{array}$ \\
\hline APP-693 (Dutch) & E693Q & Unclear & $\begin{array}{l}\text { Decreased } A \beta 42 \text { in } \\
\text { media, increased } \\
A \beta \text { aggregation, } \\
A \beta 42: A \beta 40 \text { ratio } \\
\text { decreased }\end{array}$ \\
\hline APP-693 (Arctic) & $\mathrm{E} 693 \mathrm{G}^{131}$ & Unclear & $\begin{array}{l}\text { Decreased } A \beta 40 \\
\text { and } A \beta 42 \text { in } \\
\text { plasma, } \\
\text { A } \beta 42: A \beta 40 \text { ratio } \\
\text { decreased }\end{array}$ \\
\hline APP-693 (Italian) & E693K $\mathrm{K}^{131}$ & Unclear & $\begin{array}{l}\text { Decreased } A \beta 42 \text { in } \\
\text { media, } A \beta 42: A \beta 40 \\
\text { ratio decreased }\end{array}$ \\
\hline APP-694 (Iowa) & D694N & Unclear & $\begin{array}{l}\text { Enhanced } \\
\text { fibrillization of } \\
\mathrm{A} \beta 40^{132} \\
\end{array}$ \\
\hline PS1-FAD mutations & $\begin{array}{l}\text { M139I, } \\
\text { H163A, and } \\
\text { others }\end{array}$ & $\begin{array}{l}\text { Differential } \gamma- \\
\text { secretase cut }\end{array}$ & $\begin{array}{l}A \beta 42: A \beta 40 \text { ratio } \\
\text { increased }\end{array}$ \\
\hline PS2-FAD mutations & & $\begin{array}{l}\text { Differential } \gamma- \\
\text { secretase cut }\end{array}$ & $\begin{array}{l}A \beta 42: A \beta 40 \text { ratio } \\
\text { increased }\end{array}$ \\
\hline $\begin{array}{l}\text { Trisomy } 21 \text { (Down's } \\
\text { syndrome) }\end{array}$ & & $\begin{array}{l}\text { Increased APP } \\
\text { production }\end{array}$ & $\begin{array}{l}A \beta 40 \text { and } A \beta 42 \\
\text { increased }\end{array}$ \\
\hline Apolipoprotein E4 & & $\begin{array}{l}\text { Competes for } L D L \\
\text { receptor-related } \\
\text { protein }(L R P)\end{array}$ & $\begin{array}{l}\text { Increased } A \beta \\
\text { aggregation }\end{array}$ \\
\hline
\end{tabular}


Overexpression of the mutant V717F leads to neuronal cell death, and damage to synapse termini, well before the development of amyloid plaques. ${ }^{15}$ A double mutant transgenic mouse model also expressing the Swedish FAD mutant led to synapse transmission deficits even in young mice without amyloid plaques. These observations suggest that neurotoxicity of $A \beta$ is independent of plaque formation, consistent with the neurotoxic agent being other species such as oligomers as discussed in section 2.6.1. ${ }^{15}$ A mutation M67I is found to eliminate production of $\mathrm{A} \beta 42 .{ }^{133}$ High levels of $A \beta 42$ result in age-dependent formation of amyloid plaque in FAD-mutant hAPP mice, but not in wild-type hAPP mice. ${ }^{133}$ There is no correlation between synapse damage and hAPP levels or plaque load, although there is an inverse correlation with $A \beta$ levels. This points to the neurotoxicity of $A \beta$ even in the absence of plaques. $^{133}$

$\mathrm{A} \beta$ is produced from APP via cleavage by the secretase enzymes. APP is a transmembrane protein that is postranslationally modified through the secretory pathway. The first proteolytic cleavage identified is that by $\alpha$-secretase, occurring 12 amino acids towards the $\mathrm{N}$ terminus from the transmembrane domain (Fig.7). ${ }^{\text {1a }}$ This cleavage produces the large soluble $\alpha$-APPs peptide into the extracellular space, along with release of the 83-residue C-terminal fragment $(\mathrm{CTF})$ in the membrane. Some APP molecules not subjected to $\alpha$-secretase cleavage can be cleaved by $\beta$-secretase releasing the slightly smaller $\beta$-APPs ectodomain derivative and retaining a 99residue $\mathrm{CTF}$ (C99) in the residue of the membrane. The $\beta$-secretase cleavage can be followed by $\gamma$-secretase activity to produce $A \beta$, or alternatively sequential action of $\alpha$ and $\gamma$-secretases (the latter acting on C83) produces the p3 peptide fragment (Fig.7). ${ }^{\text {1a }}$ The presenilin $/ \gamma$-secretase complex can cleave at other sites $\varepsilon$ and $\zeta$ in the 
transmembrane domain followed by the final cut at the $\gamma$-cleavage C-terminal site to produce $\mathrm{A} \beta 38, \mathrm{~A} \beta 40$ or $\mathrm{A} \beta 42 .^{6 \mathrm{e}}$

\subsubsection{A $\beta$ Mutations}

Strong evidence for the A $\beta$ hypothesis comes from genetic analyses of FAD (Section 2.2.3), since most mutations in the genes for APP, and PS1 and PS2 genes appear to cause accumulation of the $A \beta 42$ form.

The following mutations of $\mathrm{A} \beta$ are derived from those for APP (section 2.2): ${ }^{91 \mathrm{a}, 134}$ A21G Flemish, E22K Italian, E22Q Dutch, E22G arctic, D23N Iowa.

Fig.5 illustrates the location of these mutants, along with the correspondence to the APP sequence. Most mutations occur close to the $\beta$-secretase cleavage site, increasing cellular production of $A \beta 40$ and $A \beta 42$, or just after the $\gamma$-secretase cleavage which selectively increases production of the more toxic $A \beta 42$.

A systematic investigation of the aggregation tendency of all 798 single-point mutations of $A \beta 42$ was carried out using the Zyggregator algorithm (Section 4.5) to quantify aggregation propensity. ${ }^{109 \mathrm{~g}}$ Seventeen mutants were then expressed in Drosophila melanogaster and properties including in vivo toxicity (survival time) and relative locomotor ability were correlated to the aggregation propensity. Mutants involving E22G (alone, or with one other residue substitution, except I31E/E22G) are found to be most highly pathogenic. ${ }^{109 \mathrm{~g}}$ This is consistent with the observed higher rate of oligomerization and fibrillization of the arctic E22G variant compared to the wild-type peptide. $^{135}$ 


\subsection{Neuronal Toxicity of $A \beta$}

At least for human neurons, intracellular $A \beta 42$ is neurotoxic. ${ }^{91 b}$ There is still some controversy about the precise location of $\mathrm{A} \beta$ aggregation in vivo, ${ }^{\text {1a }}$ although the endoplasmic reticulum and Golgi have been identified (see Fig.6). ${ }^{41}$ A review discusses the intra-cellular production of $A \beta{ }^{41}$ A novel super-resolution fluorescence imaging technique has been used to probe $\mathrm{A} \beta 42$ fibrillization within HeLa cells (differences in fibril morphology in vivo and in vitro were also noted). ${ }^{136}$

$\mathrm{A} \beta 42$ is selectively intracellularly cytotoxic to human neurons, and not to other cell lines. ${ }^{137} \mathrm{~A} \beta 42$ but not $A \beta 40, A \beta(42-1)$ or $A \beta(40-1)$ is toxic to human neurons. ${ }^{137}$ The proaptotic proteins Bax and p53 are implicated in this intracellular toxicity. ${ }^{137-138}$ Disturbances in the cell division cycle may influence apoptosis in AD and this has been related to processing of APP and cyclin-dependent phosphorylation of tau. ${ }^{138-139}$

Long-term potentiation (LTP) is a long-lasting enhancement of synaptic efficacy after brief high frequency stimulation. It has been widely used as a model of synaptic plasticity. $A \beta 42$ and $A \beta 40$ are known to disrupt LTP in neurons. ${ }^{140}$ Neurons from transgenic mice expressing genes encoding mutant $\mathrm{APP}^{15,88,133,141}$ or presenilin linked to $\mathrm{FAD}^{1 \mathrm{a}, 1 \mathrm{~b}, 55 \mathrm{a}, 56}$ exhibit damage to synapses and dendritic spine loss. $\mathrm{A} \beta$ is implicated in these defects because $\gamma$-secretase inhibition ameliorates some indicators of synapse damage. ${ }^{141 f, 142}$ Synthetic A $\beta 42$ mediates long-term depression (LTD) in an NMDARdependent manner in vivo ${ }^{143}$ as does A $\beta$ secreted by neurons that overexpress APP. ${ }^{144}$ Deposition of amyloid plaques and deficits in LTP are observed together, although 
spine loss and decrease in pre-synaptic terminal density is observed before plaque deposition probably pointing to the influence of oligomeric or pre-fibrillar $A \beta$ on neurotoxicity. ${ }^{15,141 d}$ This is also supported by the fact that cognitive loss preceeds the observation of amyloid plaques. ${ }^{88,141 \mathrm{a}-\mathrm{c}, 145}$ Later work specifically implicated soluble oligomeric A $\beta$ in synaptoxicity and inhibition of LTP. ${ }^{140 b, 146}$ (This is also discussed in section 2.6.1).

Loss of glutamate receptors such as those for AMPA and NMDA caused by A $\beta$ is implicated in synaptic depression and dendritic spine loss. ${ }^{142,146 \mathrm{~d}, 147} \mathrm{~A} \beta$ is known to inhibit LTP, ${ }^{148}$ as do A $\beta$ fragments such as $A \beta(25-35) .{ }^{149}$ The blockage is mediated by stimulation of certain kinases. ${ }^{150} \mathrm{~A} \beta$-induced pathology may progress in a neurotransmitter-specific manner with different susceptibility for cholinergic, gluataminergic and GABAergic transmission $\left(\mathrm{GABA}=\gamma\right.$-amino butyric acid). ${ }^{151}$ $A \beta(25-35)$ was used to investigate the effect of NMDA and GABA receptor antagonists. $^{152}$

$\mathrm{A} \beta$ binds to the $\alpha 7$ nicotinic acetylcholine receptor, $\mathrm{nAChR}$, in neurons. ${ }^{153}$ This can result in receptor internalization and hence re-uptake of extracellular $A \beta{ }^{41}$ Since $\alpha 7$ $\mathrm{nAChR}$ is co-localized with $\mathrm{A} \beta$ in plaques and $\mathrm{A} \beta$ disrupts calcium activation and acetylcholine release at the receptor, this interaction may be important in $\mathrm{AD}$ pathophysiology. ${ }^{153 a} \mathrm{~A} \beta$ blocks the response of these nicotinic receptors, at least at high concentration ${ }^{154}$ (at low concentration $\mathrm{A} \beta 42$ seems to activate the $\alpha 7 \mathrm{nAChR}$, although there is still controversy concerning this). ${ }^{155}$ Thus, stimulating nicotinic receptors (eg. with nicotine) protects neurons against $A \beta$ toxicity. ${ }^{154 a, 156}$ 
Neuroprotection can also be achieved using $\alpha 7$-receptor agonists. ${ }^{154 a, 155 a}$ However, long-term use of nicotinic agonists may induce desensitization of nicotinic receptors. ${ }^{157}$ This led to the proposed use of allosteric modulators which bind to a site on nAChR distinct from that of the natural acetylcholine binding site. ${ }^{157}$ The specific sequence $A \beta(12-28)$ was implicated in the inhibition of nicotinic currents. ${ }^{158}$

GABA receptors are also potential targets to treat $\mathrm{AD}$. Activation of GABA receptors increases neuronal vulnerability to toxic damage by $\mathrm{A} \beta .^{159}$ This can be prevented by taurine (2-aminoethanesulfonic acid, related to tramiprosate, Fig.13) or GABA itself, or $\mathrm{GABA}_{\mathrm{A}}$ receptor agonists. ${ }^{152,159}$

The role of microglia in the deposition of $\mathrm{A} \beta$ plaques (Fig.8) has been examined. Microglia are support cells involved in inflammation that surround senile plaques. Their role is not completely clear, ${ }^{160}$ since it has been proposed that they can clear amyloid deposits or alternatively may contribute towards their deposition (and particularly associated inflammation). Reactive microglia associated with $\mathrm{A} \beta$ plaques are involved in inflammation in $\mathrm{AD}$. Fibrillar $\mathrm{A} \beta$ initiates a tyrosine kinase-based response in mouse microglia (and a human cell line) resulting in production of neurotoxic secretory species, proinflammatory cytokines and reactive oxygen species. ${ }^{160 \mathrm{~b}}$ The cytokine TNF- $\alpha$ generated by monocytes and microglia is responsible for most of the $A \beta$-induced neurotoxicity. ${ }^{160 \mathrm{~b}}$ Cytokine TGF- $\beta 1$ is also involved in the response to injury and has been found in the CNS of AD patients. It has been shown that TGF- $\beta 1$ induceds $A \beta$ deposition using a mouse model expressing this cytokine from astrocytes. ${ }^{161}$ 


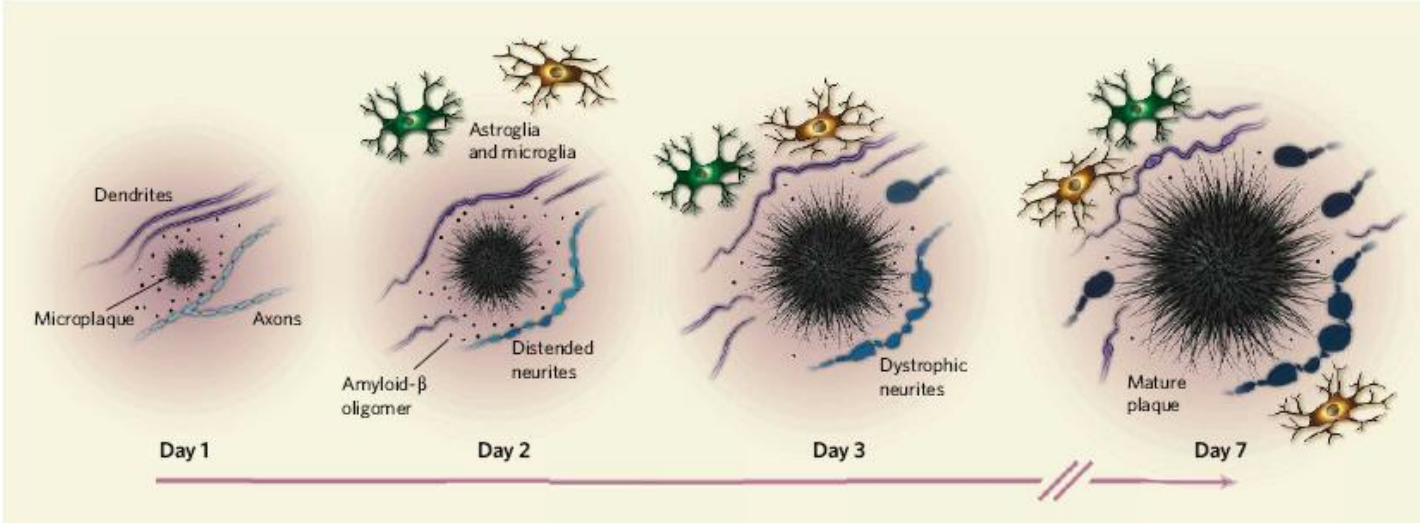

Fig.8. Mechanism of plaque formation and associated migration of glial cells, based on work by Meyer-Luehmann et al. ${ }^{162}$ Reprinted by permission from Macmillan Publishers Ltd: Nature 451, 499, copyright 2008.

Microinjection of fibrillar, but not soluble $A \beta$, in the brains of aged rhesus monkeys leads to microglial proliferation as well as loss of neurons and tau phosphorylation. ${ }^{160 \mathrm{a}}$ Fibrillar $\mathrm{A} \beta$ at plaque-equivalent concentration was found not to be toxic in the brain of young rhesus monkeys, pointing to the role of aging in promoting susceptibility towards $A \beta$ neurotoxicity. ${ }^{160 a}$ Microglia may enhance the toxicity of $A \beta$ by releasing glutamate through the cysteine-glutamate transporter system $\mathrm{x}_{\mathrm{c}}{ }^{-}$, and the neurotoxicity can be eliminated via inhibition of NMDA receptors or system $\mathrm{x}_{\mathrm{c}}{ }^{-160 \mathrm{c}}$ Microglial secretion of ApoE was found to exert a neuroprotective effect. ${ }^{160 \mathrm{c}}$ On the other hand, Nagele et al. investigated the role of microglia in $A \beta$ plaque formation and found that they can facilitate the conversion of soluble and oligomeric $\mathrm{A} \beta$ into fibrillar form, and that microglia do not remove $A \beta$ from plaques. ${ }^{163}$ These authors also highlight the role of astrocytes in accumulating $A \beta$-positive material as part of their role in debris clearance in response to localized neurodegeneration. It is also suggested that $A \beta$ fibrillization can occur within the surface plasma membrane of microglia. ${ }^{163} \mathrm{In}$ 
contrast to the findings of Nagele et al., Simard et al. showed using a transgenic mouse model that bone-marrow derived cells (which can cross the BBB) that differentiate into microglia are able to eliminate amyloid deposits by cell-specific phagocytosis. ${ }^{164}$ Using multiphoton laser confocal microscopy, Meyer-Luehmann et al. showed that microglia are activated within 1-2 days of the appearance of a new plaque and that micro-plaques are rapidly formed that eventually develop into mature plaques (Fig.8). ${ }^{160 \mathrm{~d}}$ Accumulation of microglia during inflammation can be mediated via Chemokine (C-C motif) receptor 2 (CCR2)-signaling and thus CCR2 and its main ligand CCL2 (MCP-1) might also be involved in the altered metabolism of A $\beta$ underlying Alzheimer's disease (AD). ${ }^{165}$ Cannabinoids may also have a role in neuroprotection by blocking microglial activation. ${ }^{166}$ Senile plaques express cannabinoid receptors $\mathrm{CB}_{1}$ and $\mathrm{CB}_{2}$ together with markers of microglial activation and a synthetic cannabinoid was shown to prevent $A \beta$-induced microglial activation. $^{166}$

The zinc metalloprotease insulin-degrading enzyme (IDE, insulysin) is central to the turnover of insulin and degrades $\mathrm{A} \beta$ in the mammalian brain. ${ }^{108,167} \mathrm{IDE}$ forms a stable complex with $A \beta 40$ and with $A \beta(17-27) .{ }^{168}$ IDE actually forms a complex with $A \beta$ monomer and not oligomers and so is not able to inhibit oligomer-induced loss of LTP. ${ }^{146 a}$ Monomeric but not aggregated A $\beta$ was able to associate irreversibly with IDE via the substrate binding site of the protease. ${ }^{168}$ The phosphorylation of $A \beta$ at serine residue S8 reduces its clearance via IDE and angiotensin-converting enzyme (ACE). ${ }^{169}$ The other major endopeptidase involved in $\mathrm{A} \beta$ clearance is the zinc metalloprotease neprilysin (NEP), ${ }^{170}$ although other proteases capable of degrading $\mathrm{A} \beta$ have been investigated. ${ }^{108 \mathrm{~b}, 167}$ Aggregation-mediated $\mathrm{A} \beta 42$ toxicity is decreased 
when aging is slowed (in a C. elegans model) by decreasing insulin growth factor-1like signaling (IIS), pointing to a link between the aging process and aggregationinduced neurodegeneration ${ }^{110 \mathrm{c}}$ On the other hand, the transcription factors DAF-16 and HSF-1 which express numerous chaperones (Section 3.2.2) regulate $A \beta$ aggregation and disaggregation activities respectively to promote cellular survival in response to toxic aggregation events (Fig. 9), ${ }^{110 \mathrm{c}}$ and may be the target for therapeutics. ACE plays an important role in blood pressure and body fluid regulation and sodium homeostatis. It is associated with AD in the Japanese population. ${ }^{171}$ ACE is found to inhibit $A \beta$ aggregation and can degrade it by cleavage at N7-S8. ${ }^{171 b}$

Figure 9 shows schematically proposed pathways for in vivo aggregation of $A \beta 42$, relevant to age-related proteotoxicity. ${ }^{110 \mathrm{c}}$ The IIS pathway is regulated by the receptor DAF-2 (inhibition of DAF-2 expression extends the lifespan of C. elegans worms). The transcription factors heat shock factor (HSF-1) and DAF-16 regulate opposing disaggregation and aggregation processes. The preferred mechanism whereby toxic aggregates are rapidly degraded (5-II) is positively regulated by HSF-1 (stage 5-A) and negatively regulated by DAF-2 (stage 5-C). When the HSF-1-regulated dissaggregation mechanism is overloaded, a second comes into play (5-III). This produces less toxic higher $M_{\mathrm{w}}$ aggregates. This is positively regulated by DAF-16 (stage 5-B) and negatively by DAF-2 (stage 5-D). The high $M_{\mathrm{w}}$ aggregates can be eliminated by several methods indicated in the scheme. 


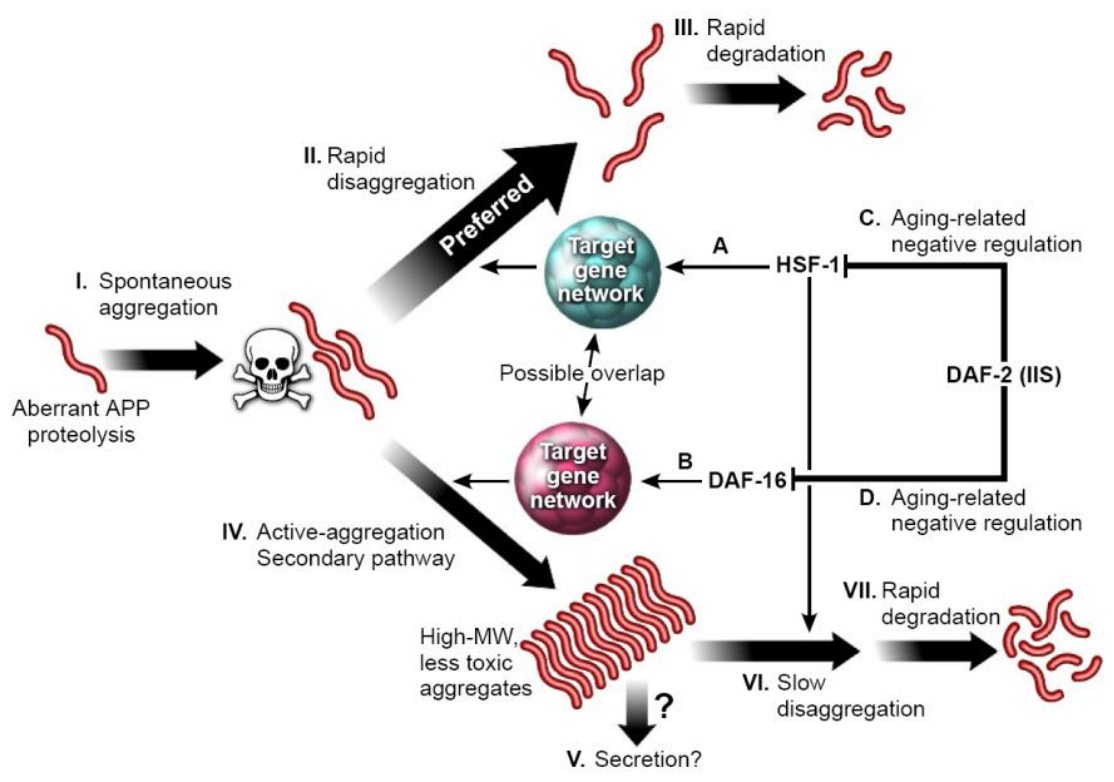

Fig.9. Pathways of regulation of fibrillization in age-onset $A \beta$ proteolysis. ${ }^{110 c}$

Adapted from Cohen, E. et al., Science 2006, 313, 1604. Courtesy of Ehud Cohen.

\subsection{Biomarkers for AD}

Biomarkers for $\mathrm{AD}$ are expected to improve the accuracy of diagnosis and to assist in differentiation of cases involving changes in $\mathrm{A} \beta$ metabolism. Biomarkers can also be used to investigate the influence of drugs on $A \beta$ production (theranostics) along with safety monitoring, eg. of inflammatory responses in the case of adverse effects. ${ }^{26} \mathrm{~A}$ major public-private partnership initiative funded by the $\mathrm{NIH}$, non-profit $\mathrm{AD}$ research organizations and major international pharma companies is the Alzheimer's Disease Neuroimaging Initiative (ADNI), which aims to identify biomarkers in volunteer patients. ${ }^{172}$ 
Different biomarkers may be appropriate during the progression of neurodegeneration towards AD leading to a dynamic model for applicable biomarkers as shown in Fig. $10 .^{173}$

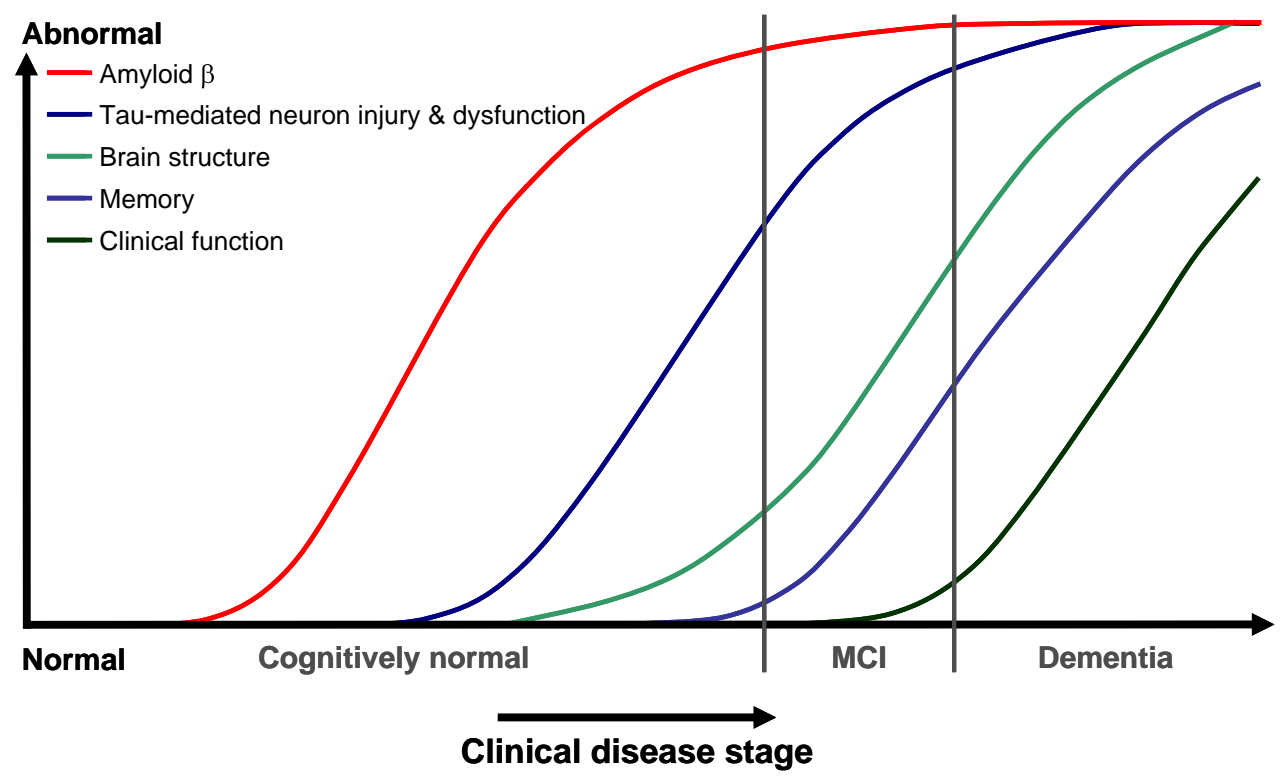

Fig.10. Dynamic events as a basis for use of biomarkers. ${ }^{173} \mathrm{~A} \beta$ is identified by CSF A $\beta 42$ or PET amyloid imaging. Tau-mediated neuronal injury and dysfunction is identified by CSF tau among other indicators. Brain structure is probed using structural MRI. MCI = mild cognitive impairment. ${ }^{173}$ Reprinted from Jack, C. R. et al., The Lancet Neurology, 2010, 9, 119. Copyright 2010, with permission from Elsevier.

Research on biomarkers in CSF and plasma has recently been reviewed. ${ }^{26,173-174}$ The only established biomarkers are three that can be assayed in CSF: A $\beta 42$, total tau ( $\mathrm{t}$ tau) and p-tau (phosphorylated tau, at position threonine 181 or threonine 231 ) ${ }^{19,175} \mathrm{~A}$ combined analysis of two or more these biomarkers accurately diagnoses AD more accurately than a single one. ${ }^{26}$ The combination of these three analytes has high 
predictive value for prodromal (early symptomatic) AD. ${ }^{26}$ Reduced CSF A $\beta 42$ (and $\mathrm{A} \beta 40^{176}$ ) levels in cognitively normal elderly people who later developed AD have been observed from population-based studies ${ }^{176-177}$ and clinical studies. ${ }^{178}$ No changes were observed in CSF t-tau or p-tau. ${ }^{176-177}$ Biomarkers for tau phosphorylation appear specific to $\mathrm{AD}$ in contrast to changes in total tau and $\mathrm{A} \beta 42$ which are found in patients with other neurodegenerative diseases. ${ }^{26}$ An analysis of multiple study populations to examine potential CSF biomarkers for AD revealed that these three biomarkers can be used as diagnostics to predict incipient $\mathrm{AD}$ in patients with mild cognitive impairment. ${ }^{19,175,179}$ A total of eighteen signalling proteins have been identified in plasma that can predict for $\mathrm{AD}$, the study being based on plasma samples from individuals with presymptomatic to late-stage Alzheimer's disease and from controls. $^{180}$

Due to the need for invasive treatment (lumbar puncture) associated with measurements using CSF, reliable biomarkers in blood are also sought. It has been suggested that $A \beta(1-42)$ plasma levels are not a sensitive and specific indicator for early diagnosis. ${ }^{26,174 \mathrm{~b}}$ This was ascribed to (i) the fact that plasma $A \beta$ is derived from peripheral tissues and not the brain, (ii) variations in $\mathrm{A} \beta$ levels due to time-dependent fluctuations, (iii) binding of $A \beta$ to other proteins, (iv) the influence of medications and (v) the involvement of APP and A $\beta 40$ in platelet aggregation. ${ }^{26,174 b}$ However, it has been reported that a reduction in plasma $A \beta 42 / A \beta 40$ ratio is associated with cognitive decline over 9-10 years. ${ }^{181}$ Tau-related enzymes have been studied as potential blood biomarkers, including kinases involved in tau hyperphosphorylation. ${ }^{174 \mathrm{~b}}$ However, it has been concluded that currently tau-related 
biomarkers are not reliable diagnostics. ${ }^{174 \mathrm{~b}}$ Several other potential blood biomarkers are discussed by Blennow et al. ${ }^{26}$

Antibodies associated raised against $A \beta$ (discussed further in Section 2.5) may be useful biomarkers. ${ }^{172 a}$ A recent study reports the development of immunoglobulin $\mathrm{G}$ biomarkers for $\mathrm{AD}$ via a screening study using synthetic oligomeric peptoids to capture antibodies. ${ }^{182}$

Biomarkers associated with inflammation have also been investigated, in particular proinflammatory cytokines. ${ }^{174 \mathrm{~b}}$ Other disease-related biomarkers include ubiquitin and biomarkers related to cellular senescence such as p53 conformational state or telomere shortening. ${ }^{174 \mathrm{~b}}$ Finally, there are biomarkers associated with cerebrovascular damage. ${ }^{174 \mathrm{~b}}$ Other candidate CSF biomarkers include BACE, APP isoforms, truncated $\mathrm{A} \beta$ isoforms, $A \beta$ oligomers, endogenous $A \beta$ antibodies and neuronal and synaptic markers. ${ }^{26,174 a}$ In particular, $\mathrm{A} \beta$ oligomers are promising biomarkers, however low CSF concentrations make sensitive detection a challenge. ${ }^{174 a}$ Recently, the (small cytokine) chemokine (C-C motif) receptor 2 (CCR2) has been proposed as a CSF biomarker. $^{165}$

Methods to identify biomarkers for AD diagnosis based on different ELISA assays, mass spectrometry, DNA and gene chips etc are also discussed elsewhere. ${ }^{174 \mathrm{~b}}$

Imaging methods such as MRI (magnetic resonance imaging), fMRI (functional MRI) and PET (positron emission tomography) to diagnose AD are reviewed elsewhere. ${ }^{173-}$ ${ }^{174}$ The use of A $\beta$ ligands for PET imaging has attracted attention, notably Pittsburgh 
compound B (PIB, Fig.11b). This benzothiazole is derived from the well-known amyloid binding compound thioflavin T (Fig.11a) and it enables direct visualization of fibrillar A $\beta$ load in the brain of living patients. ${ }^{183}$ Another widely used PET reagent is FDG, $\left[{ }^{18} \mathrm{~F}\right]$-2-deoxy-D-glucose, which is sensitive to neuronal glucose metabolism. ${ }^{174 a, 183 d}$ Investigation of changes in the retina related to neurodegeneration, i.e. the monitoring of nerve cell death using in vivo cell marker methods, has been proposed as a method to screen for AD. ${ }^{184}$ Indeed, A $\beta$ is deposited also in the retina.

(a)<smiles></smiles>

Thioflavin T (b)<smiles>C=[V]c1ccc(-c2nc3ccc(O)cc3s2)cc1</smiles>

PIB

Fig.11. (a) Thioflavin T, (b) Pittsburgh-compound B (PIB) used as a tracer in PET imaging.

The SERPIN (serine protease inhibitor) $\alpha_{1}$-antichymotrypsin has been shown to be a biomarker for Alzheimer's, and is present in CSF. ${ }^{185}$ This is probably due to the role of oxidative stress and inflammation in Alzheimer's, specifically in the overproduction of secretase, ${ }^{118-119}$ which in turn is correlated with $\mathrm{A} \beta$ load. ${ }^{186}$

\subsection{Antibodies to $A \beta$ and Sequences Therein}

Table 3 lists antibodies raised against $A \beta$ and $A \beta$ sequences. These are widely used in studies to identify particular $\mathrm{A} \beta$ species produced in vivo. 
Table3. Commonly used monoclonal A $\beta$ antibodies. Developed from ref. ${ }^{40}$

\begin{tabular}{|c|c|c|}
\hline Name & Epitope & Ref. \\
\hline $2 \mathrm{G} 3$ & $\begin{array}{l}A \beta(33-40) \\
A \beta(31-40) \\
\text { Specific for } A \beta 40\end{array}$ & $\begin{array}{l}161 \\
187 \\
188\end{array}$ \\
\hline 3D6 & $\mathrm{A} \beta(1-5)$ & 161,187 \\
\hline $4 \mathrm{G} 8$ & $\begin{array}{l}A \beta(17-24) \\
A \beta(17-28)\end{array}$ & $\begin{array}{l}87,189 \\
24\end{array}$ \\
\hline 6C6 & $\mathrm{A} \beta(1-16)$ & $106 a, 190$ \\
\hline $6 \mathrm{E} 10$ & $\begin{array}{l}A \beta(1-16) \\
A \beta(1-17) \\
A \beta(4-9) \\
A \beta(4-13)\end{array}$ & $\begin{array}{l}24,189 \mathrm{c} \\
189 \mathrm{a} \\
191 \\
168,192\end{array}$ \\
\hline $8 \mathrm{~F} 5$ & $\begin{array}{l}\text { A } \beta 42 \text { globulomers } \\
\text { (docademeric } \\
\text { oligomers) }\end{array}$ & 193 \\
\hline 10D5 & $\begin{array}{l}A \beta(1-28) \text { epitope } \\
A \beta(x-12)\end{array}$ & 161 \\
\hline $14 \mathrm{C} 2$ & $\mathrm{~A} \beta(33-40)$ & $190 \mathrm{~b}$ \\
\hline $14 \mathrm{C} 12$ & $\mathrm{~A} \beta(13-28)$ & $190 \mathrm{~b}$ \\
\hline 21F12 & $\begin{array}{l}\text { A } \beta(33-42) \\
\text { A } \beta 42 \text { specific }\end{array}$ & $\begin{array}{l}187 \\
188 \mathrm{~b}\end{array}$ \\
\hline 266 & $\begin{array}{l}\mathrm{A} \beta \text { "central } \\
\text { domain" } \\
\mathrm{A} \beta(13-28)\end{array}$ & $\begin{array}{l}103,106 a, 188 a, 189 b \\
106 a, 190 a\end{array}$ \\
\hline
\end{tabular}

Several polyclonal antibodies are also used, as listed for instance by Walsh et al. ${ }^{40}$

An antibody, now known as A11, that recognises an epitope that is displayed specifically by soluble oligomers of many polypeptides has been identified. ${ }^{194}$ The antibodies were raised in rabbits to an oligomeric model antigen comprising gold nanoparticles grafted with $A \beta 40$. This antibody inhibits the toxicity of $A \beta 40$ and A $\beta 42$ oligomers. ${ }^{194 a}$ This antibody also recognises oligomers from a range of other proteins and peptides. ${ }^{194 \mathrm{a}}$ Recognition was not observed for low $M_{\mathrm{w}^{-}}$or fibrillar- A $\beta$ 
species. This indicates that the antibody recognises a common epitope in soluble oligomers. An antibody termed 8F5 is raised specifically against so-called A $\beta 42$ globulomers (docademeric oligomers). ${ }^{193}$ An antibody, distinct from A11, that recognizes on-pathway oligomers (i.e. pre-fibrillar oligomers) called OC has also been reported. ${ }^{194 b, 194 \mathrm{c}}$ An antibody against A $\beta(4-10)$ (FRHDSGY) inhibits A $\beta$ fibrillization and cytotoxicity, without generating an inflammatory response. ${ }^{195}$ Similarly, an antibody against $A \beta(1-11)$ prevents aggregation of $A \beta 42$ and causes disaggregation of preformed A $\beta 42$ fibrils. ${ }^{196}$ The binding of fluorescently labeled antibody 6E10 to amyloid deposits within the TgCRND8 mouse brain has been observed, up to $2.5 \mathrm{~mm}$ away from the site of injection. ${ }^{197}$

A number of monoclonal antibodies raised against oligomers and fibrils have been identified, which have activity against binding of $\mathrm{A} \beta$ to cells and reactive oxygen species generation. ${ }^{198}$ Antibodies that recognize the $\mathrm{N}$ or $\mathrm{C}$ terminal residues of $\mathrm{A} \beta 42$ have been used in immunological studies using soluble dimers. ${ }^{199}$ A solution NMR structure of the complex formed between $\mathrm{A} \beta 40$ and an affibody protein $\mathrm{Z}_{\mathrm{A} \beta 3}$ indicates that this affinity ligand protein stabilizes the $\beta$-sheet structure, and the hairpin observed in $A \beta 40$ (section 4.1) is retained. ${ }^{200}$ Coexpression of $Z_{A \beta 3}$ with $A \beta 40$ and A $\beta 42$ can be used to produce both isoforms recombinantly. ${ }^{201}$

\subsection{Oligomers}

\subsubsection{Toxicity of Oligomers}

It is now thought that oligomers formed in the initial self-assembly process of $A \beta$ are the toxic agents. ${ }^{2 \mathrm{a}, 2 \mathrm{~d}, 6 \mathrm{~d}, 39,91,104,145 \mathrm{a}, 194 \mathrm{a}, 202}$ Evidence for this comes from several 
experiments on disease related and non-disease related proteins. In vivo and cell culture experiments showed that $\mathrm{A} \beta 42$ oligomers, formed under conditions that inhibited fibril formation, were neurotoxic. ${ }^{91 a, 145 a, 202 a}$ Synthetic A $\beta$ oligomers inhibit long term potentiation of neuronal cells, as discussed in more detail in section 2.3. It is possible to obtain naturally excreted SDS-stable oligomeric forms of $A \beta$ and extract them from the conditioned medium of 7PA2 Chinese Hamster Ovary cells. $^{40,105,146 a, 146 \mathrm{~d}, 189 \mathrm{~b}}$ The oligomers are produced soon after generation of human $\mathrm{A} \beta$ in intracellular vesicles in CHO cells which express an APP isoform. The intercellular production of oligomers, principally dimers, was inferred from previous studies using primary human neurons. ${ }^{40}$ The secreted oligomers (predominantly dimers and trimers in the conditioned medium, i.e. released from the microsomes) disrupt the LTP of rat hippocampal neurons ${ }^{146 a, 146 c, 146 \mathrm{~d}}$ as discussed further in section 2.3. These oligomeric forms of $A \beta$ were also shown to disrupt the learning behaviour of rats. ${ }^{104}$ The soluble oligomers induce tau hyperphosphorylation leading to disruption of the microtubule skeleton and neuritic degeneration. ${ }^{199}$

Further evidence in support of the toxic oligomer hypothesis comes from the observation that molecules that stabilize fibrils by accelerating $A \beta$ fibril formation leads to a loss of inhibition of LTP by A $\beta$ oligomers. ${ }^{191}$ An orcein-related small molecule $\mathrm{O} 4$ was found to bind to hydrophobic residues in $\mathrm{A} \beta$ and to promote the formation of $\beta$-sheet rich fibrils whilst decreasing the concentration of oligomers. ${ }^{191}$

Anti-A $\beta$ antibodies isolated from immunoglobulin strongly disrupt fibrillization. ${ }^{203}$ Experiments using polyclonal antibodies indicate that they suppress the toxicity of soluble oligomers whereas there is no antibody response to mature fibrils. ${ }^{194 a}$ This has 
been proposed as a route to vaccination using $A \beta 42$ oligomers. ${ }^{204}$ Experiments on $A \beta$, $\alpha$-synuclin and transthyretin suggest that cytoxicity shares a common cause not related to the specific sequence. ${ }^{39 a, 39 c}$ Recent in vivo studies using a mouse model suggest that specific soluble $A \beta$ multimeric species are associated with memory loss in Alzheimers, specifically dodacameric $56 \mathrm{kDa}$ species. ${ }^{87}$

Inhibition of $\gamma$-secretase can prevent oligomer formation and restore LTP of rat neurons. ${ }^{146 a}$ Two $\gamma$-secretase inhibitors (flurbiprofen and semagacestat, Fig.13) recently reached, but failed, phase 3 trials. Possible reasons for the failure of these trials that do not necessarily invalidate the $A \beta$ hypothesis have been discussed. ${ }^{3,49}$ The $\gamma$-secretase inhibitor semagacestat developed by Eli Lilly ${ }^{205}$ also failed phase 3 trials due to low potency and signs of apparent Notch toxicity including gastrointestinal symptoms and skin cancer. ${ }^{3,49}$

$\mathrm{A} \beta$ oligomers adversely affect synapse function. ${ }^{140 \mathrm{~b}, 206}$ This leads to the damage to neuropili believed to underlie $\mathrm{AD} .^{206} \mathrm{~A} \beta$ oligomers may inhibit long-term potentiation and facilitate long-term depression, ${ }^{147 \mathrm{~b}}$ depending on the extent of change in the calcium ion concentration, although there is still controversy around this issue. This has been associated with the synaptic removal of AMPA receptors (AMPARs). ${ }^{140 \mathrm{~b}}$ Soluble oligomers, whether naturally secreted or prepared from synthetic $A \beta$, inhibit hippocampal long-term potentiation, ${ }^{146 a, 207}$ due to removal of AMPA receptors ${ }^{140 \mathrm{~b}}$ and disruption of neuronal glutamate uptake. ${ }^{147 \mathrm{~b}}$ They also cause a rapid decrease in membrane expression of memory-related receptors such as NMDA and EphB2. ${ }^{206}$ 
The importance of "gatekeeper residues" that cap aggregation prone sequences in natural proteins and help to hinder aggregation into fibrils has been highlighted. ${ }^{202 \mathrm{c}}$ There is clearly scope for evolutionary pressure to ensure that proteins contain residues that hinder aggregation and/or promote folding into the native state. ${ }^{202 c, 208}$ Specific residues that oppose aggregation were analysed using a computer algorithm that analyses sequence aggregation propensity, including those in $A \beta$ as discussed further in Section $4.5 .^{202 \mathrm{c}}$

Several characteristics of the AD phenotype can only be replicated using oligomers, including synaptic loss, hippocampal synaptic plasticity, microgliosis and tau hyperphosphorylation. ${ }^{3,49}$ The presence of oligomers trapped within plaques, points to the dynamic equilibrium that may exist between these species. Oligomers are formed intracellularly in human neurons. ${ }^{40}$ They appear mainly in the form of dimers. ${ }^{40}$ Oligomers influence synaptic plasticity and impair LTP in brain tissue. ${ }^{6 e, 140 b, 146,188 b}$ The molecular conformation of a highly synaptotoxic $A \beta$ oligomer structure has recently been eludicated using ssNMR. ${ }^{209}$ This study revealed that the oligomer formation is controlled by an $\mathrm{N}$-terminal $\beta$-strand.

Stable SDS-resistant oligomers have been detected in normal and AD brain. ${ }^{210}$ Oligomers comprising dimers and trimers were detected in tissue extracted from AD brain, ${ }^{210 \mathrm{~b}}$ and AFM revealed that these structures comprise 3-4 nm diameter ellipsoids. A $\beta$-Derived Diffusible Ligands (ADDLs) formed by A $\beta 42$ have been imaged by liquid state AFM. ${ }^{211}$ AFM has also been used to compare the formation of oligomer-like species and protofibrils by $\mathrm{A} \beta 40$ and $\mathrm{A} \beta 42 .{ }^{212} \mathrm{AFM}$ has also been used 
to image the oligomer-induced formation of membrane pores, as discussed further in section 2.7 .

\subsubsection{Types of Oligomers}

Various types of oligomeric species have been identified, such as protofibrils, paranuclei, globulomers or so-called A $\beta$-Derived Diffusible Ligands (ADDLs), ${ }^{146 \mathrm{~d}}$ especially in the early literature which is not reviewed in detail here. Distinctions between these species have been summarized. ${ }^{1 \mathrm{c}, 6 \mathrm{e}, 213}$ ADDLs are larger (4-6 nm diameter) structures than "low $n$ " (where $n$ is the number of associated monomers) oligomers or globulomers. ${ }^{213}$ ADDLs are thought to comprise mixtures of monomer and heterogeneous higher $n$ oligomers. ${ }^{1 \mathrm{c}}$ The observation of these oligomeric structures depends on how synthetic $A \beta$ is prepared and incubated. More recently, as discussed below, protocols to prepare oligomers from synthetic $A \beta$ in a controlled and reproducible manner, or in the secreted medium of a rodent cell line (7PA2), have been described. ${ }^{146 \mathrm{~d}}$ The natural A $\beta$ oligomers are resistant to SDS and insulindegrading enzyme (IDE) which can only digest monomeric $A \beta{ }^{6 e}$ All soluble oligomers display a common structure against antibodies raised against them (A11 antibody, section 2.5). ${ }^{194 a}$

Stable globular oligomers termed globulomers can be prepared by careful preparation methods starting from $A \beta$ monomers. Different groups report various protocols to prepare stable oligomers. ${ }^{193,214}$ Using synthetic A $\beta$, Kayed and coworkers prepare oligomers by controlled evaporation of HFIP which is used to disperse $A \beta$ into monomeric form ${ }^{194 a, 215}$ followed by redispersion in water, or dissolving the peptide in $\mathrm{NaOH}$ and diluting this stock solution in a PBS solution containing sodium 
azide. ${ }^{215 b, 216}$ Chromy et al. reported a related method to prepare stable oligomers from synthetic peptides. ${ }^{214}$ As mentioned in Section 2.6.1, the 7PA2 CHO cell line expressing mutant V717F APP has been developed to secrete $A \beta$ in oligomeric form. ${ }^{189 b, 213}$ The oligomers produced by Chromy et al. are neurotoxic ${ }^{214}$ and block LTP ${ }^{193}$ Electrophoresis in denaturing gels revealed a spectrum of oligomers including dimers, trimers, tetramers, pentamers, and higher order oligomers up to 24-mers. ${ }^{217}$

Globulomers can be prepared by incubation in the presence of SDS or fatty acids. 193,218 These oligomers appear to be dodecameric species with a mass of $60 \mathrm{kDa}$. They raise oligomer-specific antibodies $8 \mathrm{~F} 5$ (cf. Section 2.5). ${ }^{193}$ They could be related to the brain-derived soluble $A \beta^{*} 56$ dodecamers. ${ }^{6 e, 207}$ Solution NMR has been used to characterize $\mathrm{A} \beta(\mathrm{M} 1-42)$ globulomers indicating a mixed parallel and antiparallel configuration ${ }^{219}$ and a dimeric state. To confirm that the globulomers (oligomers) comprise repeats of the dimer, a mutant peptide with L17C, L34C substitution to enable disulfide crosslinking was prepared. This was found to bind to anti-oligomer antibodies with the same affinity as the WT peptide. ${ }^{219}$ The mixed $\beta$-sheet configuration is in contrast to fibrils which contain only parallel $\beta$-sheets (Section 4.1). A $\beta$ oligomers can be used to cross-seed tau oligomers. ${ }^{215 b}$

Electrospray mass spectrometry has been used to probe oligomeric states, in particular via analysis of arrival time distributions which can distinguish distinct species with the same charge/mass ratio due to differences in cross-sections of the ions generated. ${ }^{217}$ This technique reveals that unfiltered solutions of $A \beta 42$ contain monomers and large oligomers. ${ }^{217}$ Filtration can be used to isolate smaller oligomers - dimers up to dodecamers, the latter being proposed as the species that initiate 
fibrillization. ${ }^{217}$ Later, the same ion mobility mass spectrometry method was applied to investigate oligomer formation by $\mathrm{A} \beta 42$ with a comparative study to $\mathrm{A} \beta 40 .{ }^{220}$ For A $\beta 42$, oligomers up to dodecamers were observed whereas for $A \beta 40$ only oligomers up to tetramers were found. The authors proposed different mechanisms of fibril nucleation based on these observations (Fig.12). ${ }^{220}$ These distinct aggregation mechanisms were supported by earlier conclusions from experiments using photoinduced cross-linking of unmodified proteins (PICUP) ${ }^{221}$ to cross-link oligomers which were analysed using a variety of sizing techniques. ${ }^{221 \mathrm{a}}$ These studies showed that the formation of monomers up to tetramers only are observed for $A \beta 40$, whereas pentamer/hexamer paranuclei are formed preferentially by $\mathrm{A} \beta 42$.

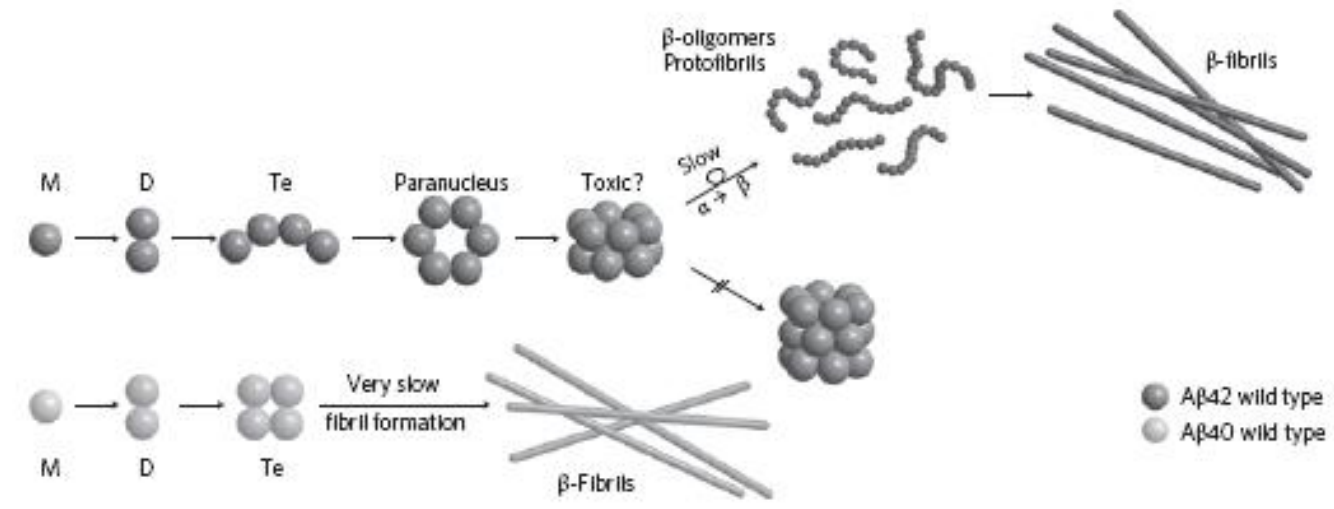

Fig.12. Distinct mechanisms of aggregation of $A \beta 42$ and $A \beta 40$ proposed by Bernstein et al. ${ }^{220} \mathrm{M}$ denotes monomer, D dimer, Te tetramer and the initial toxic species for $A \beta 42$ is proposed to be the dodecamer whilst the planar hexamer serves as a paranucleus. Reprinted by permission from Macmillan Publishers Ltd: Nature Chemistry 1, 326, copyright 2009. 
Single molecule fluorescence methods indicate that in vitro $\mathrm{A} \beta 40$ forms a heterogeneous distribution of small oligomers (from dimers to 50 -mers). ${ }^{222}$ The oligomers represent about $1 \%$ of the total number of species present, at the concentrations examined ( $30 \mathrm{nM}$ to $2 \mu \mathrm{M}$ ). Oligomers have been shown, by SAXS, SDS-page and Western blotting, to bind to APP. ${ }^{223}$ Dimers of A $\beta$ cause APP to dissociate from the native homodomer conformation into monomers, whereas $A \beta$ oligomers bind to APP but its homodimer structure is preserved. ${ }^{223}$

\subsection{Ion Channel Hypothesis}

The mechanism of $A \beta$ cytotoxicity may be due to its ability to form membrane pores or channels. $^{2 \mathrm{~d}, 58 \mathrm{a}, 224}$ This could be due to the exposure of hydrophobic regions in misfolded proteins such as those that form amyloid fibrils. ${ }^{224 b}$ Positive charge on a peptide that enables interaction with negatively charged lipid membranes may also be important. ${ }^{24 \mathrm{~b}}$ According to the channel hypothesis, pore formation is responsible for the neurotoxicity of A $\beta$. The original work by the group of Arispe et al. ${ }^{225}$ established that $\mathrm{A} \beta$ is capable of forming membrane channels.

The oligomeric form of $\mathrm{A} \beta$ is implicated in pore formation. ${ }^{24 \mathrm{~g}, 226}$ Lambert et al . showed that $\mathrm{A} \beta 42$ oligomers bind to cell membranes and cause cytotoxicity under conditions in which mature fibrils do not form. ${ }^{202 a}$ Further support for this is the finding that pore formation is inhibited by Congo red binding, ${ }^{227}$ indicating that the $\mathrm{A} \beta$ needs to be aggregated into protofilaments/oligomers for this mechanism to be effective. $A \beta$ forms pores in lipid membranes that contain multimers of the protein, as 
revealed by $\mathrm{AFM}^{226 a}$ Evidence for pore formation in vivo has also been obtained via TEM on neuronal cell membranes. ${ }^{228}$

Uptake of $\mathrm{Ca}^{2+}$ across the ion channels leads to neuronal degeneration in a dose- and time- dependent manner and ultimately cell death. ${ }^{226 a}$ Changes in the calcium level and the morphology of cultured cells was also found to be sensitive to the aggregation state of $\mathrm{A} \beta 42 .{ }^{229} \mathrm{~A} \beta$ pore formation leading to an increase in intracellular calcium has been linked to depletion of synaptic vesicles and hence blocked neurotransmission. ${ }^{230}$ Transient $\mathrm{Ca}^{2+}$ currents are observed near $\mathrm{A} \beta$ plaques in the brain of transgenic mice suggesting the presence of clusters of "hyperactive" neurons. ${ }^{231}$ A correlation between an increase in calcium ion production and a decrease in $\mathrm{A} \beta$ production has also been noted in studies on SERCA (sarco ER $\mathrm{Ca}^{2+}$ ATPase) which is a calcium channelforming protein in the endoplasmic reticulum (ER) membrane. ${ }^{232}$ Down-regulation of SERCA leads to increased $\mathrm{Ca}^{2+}$ and reduced $\mathrm{A} \beta$ levels and over-expression leads to increased $A \beta$ production. SERCA activity was also shown to be decreased in fibroblasts lacking the PS1 and PS2 presenilin genes. ${ }^{232}$

\subsection{Interaction of $A \beta$ with tau}

The tau protein is involved in microtubule assembly and stabilization within the cytoskeleton (in particular in F-actin fibrils). Mutations can lead to filamentous deposits which have been observed for several neurodegenerative diseases such as Pick's disease, Parkinsonism-dementia complex of Guam etc. ${ }^{2 a}$ Filamentous tau deposits are invariably present even in the absence of $\mathrm{A} \beta$ deposits and it is not clear in the context of fibril deposition precisely how $\mathrm{A} \beta$ and tau interact although there seems to be a synergistic effect which enhances actin bundling and 
neurodegeneration. ${ }^{2 \mathrm{a}}$ Neuronal degeneration induced by tau has been studied in vivo, although this is outside the scope of the current review. However, tau can influence $\mathrm{A} \beta$-induced neuronal dysfunction, as exemplified by a study using transgenic mice that express APP along with tau. ${ }^{4 a}$ Synergistic interactions between A $\beta$, tau and $\alpha-$ synclein can accelerate neuropathology and cognitive decline, as indicated by a study using transgenic mice. ${ }^{233}$ These authors note that the aggregation of $\alpha$-synuclein into Lewy bodies is a pathology associated with up to $50 \%$ of AD cases.

A correlation between an increase in CSF tau and ptau-181 and the amount of cortical amyloid has been reported via brain imaging studies using the PIB biomarker (Section 2.4). ${ }^{234}$ There is an inverse relationship involving cortical PIB binding, i.e. A $\beta 42$ deposition is inversely related to CSF A $\beta 42$ levels, but this is not true for plasma species. $^{183 \mathrm{c}, 234}$

\section{THERAPEUTIC TREATMENTS}

\subsection{Existing treatments}

The only drugs currently available do not cure AD but may delay the development of symptoms. The current standard of care for mild to moderate AD includes treatment with acetylcholinesterase inhibitors to improve cognitive function and memantine, an NMDA antagonist. The acetylcholinesterase inhibitors include galantamine, an alkaloid available commercially as Reminyl (Shire), Razadyne (Janssen), rivastigmine (Exelon, Novartis) and donepezil (Aricept, Pfizer). ${ }^{8,31,235}$ The patent on the latter drug expired in $2010,{ }^{236}$ however it has recently been shown that it may also be effective in the treatment of moderate-to-severe AD as well as mild-to-moderate symptoms. ${ }^{237}$ The cholinesterase inhibitor tacrine is rarely used due to poor oral bioavailability and 
several potential adverse drug reactions. ${ }^{31}$ The NMDA receptor antagonist memantine developed by Eli Lilly is also available under trade names including Ebixa (Lundbeck) and Namenda (Forest). ${ }^{8,31}$ The development of the market for these drugs (sales forecasts) has been assessed - sales were $\$ 4$ billion in $2005{ }^{8}$

Although developed as an acetylcholinesterase inhibitor, galantamine also acts to inhibit $A \beta$ aggregation. ${ }^{238}$ It is also known as an allosteric modulator of nicotinic receptors. ${ }^{239}$ Memantine is thought to function therapeutically as an open-channel blocker of NMDA receptors, ${ }^{240}$ and also attenuates the ADDL-induced increase in intraneuronal calcium. $^{241}$

\subsection{Inhibitors of Fibrillization/Oligomerization}

\subsubsection{Small Molecules}

Since there have been a very large number of papers on small molecule inhibitors of A $\beta$ fibrillization (also $\gamma$-secretase inhibitors) we are not able to review all of them. Reviews on this topic are also available. ${ }^{242}$ Here, we focus on key classes of compounds and individual compounds that have attracted particular interest, for example moving to advanced stage clinical trials. Other reviews cover many more of the compounds researched to date. The main focus in the following is on $A \beta$ fibrillization inhibitors, as this has been the main focus of small molecule inhibitor approaches. ${ }^{189 \mathrm{c}, 216,242 \mathrm{a}, 242 \mathrm{~b}, 242 \mathrm{~d}}$ However, some compounds have been developed to inhibit $\beta$-secretase or $\gamma$-secretase. ${ }^{242 a}$

A large number of small molecules have been studied for their ability to influence $A \beta$ aggregation and toxicity. ${ }^{216,242}$ Possibly the most high profile work has been on 
tramiprosate (Alzhemed, 3-amino-1-propanesulfonic acid, Fig.13) which reached phase III trails, which however were not successful. ${ }^{3,49}$ This compound is a glycosaminoglycan (GAG) mimetic (vide infra) shown to bind to soluble $\mathrm{A} \beta 40$ and $\mathrm{A} \beta 42$ and to maintain them in a non-fibrillar form. ${ }^{242 \mathrm{~d}, 243}$ It also decreases $A \beta 42$ induced neurotoxicity, is able to cross the blood-brain barrier (BBB) and, using a TgCRND8 mouse model, can reduce amyloid plaque and cerebral levels of A $\beta 40$ and $\mathrm{A} \beta 42 .^{243 a, 244}$

(a)<smiles>Oc1c(I)cc(Cl)c2cccnc12</smiles>

Clioquinol

(c)<smiles>O[C@H]1[C@H](O)[C@H](O)[C@H](O)[C@@H](O)[C@H]1O</smiles>

Scyllo-inositol

(e)<smiles>CC(C)[C@H](O)C(=O)[C@H](C)C(=O)N[C@H]1C(=O)N(C)CCc2ccccc21</smiles>

Semagacestat (b)<smiles>NCCCS(=O)(=O)O</smiles>

Tramiprosate

(d)<smiles>CC(C(=O)O)c1ccc(-c2ccccc2)c(F)c1</smiles>

Flurbiprofen

(f)<smiles>O=S(NC(CO)C(C(F)(F)F)C(F)(F)F)c1ccc(Cl)s1</smiles>

Begacestat

Fig.13. Therapeutic compounds for AD. 
A number of dye compounds have been investigated in terms of their effect on $A \beta$ aggregation and neurotoxicity. ${ }^{196}$ Congo red (CR), an amyloid-staining dye, has been the subject of several studies in this regard. ${ }^{245} \mathrm{CR}$ is found to inhibit fibril formation and neurotoxicity towards rat hippocampal cells. ${ }^{245 \mathrm{~b}}$ NMR and light scattering suggest that it binds to $\mathrm{A} \beta 40$ monomers $^{245 e}$ and AFM indicates that this ultimately leads to a distinct aggregation pathway. ${ }^{245 f}$ However, CR is potentially toxic due to the metabolic release of benzidine, and has poor BBB permeability. ${ }^{245 \mathrm{~d}} \mathrm{CR}$ has been found by Podlisny et al. to inhibibit the oligomerisation of $\mathrm{A} \beta 40$ using the $\mathrm{A} \beta$ expressed by 7PA2 cells in conditioned medium (see Section 2.6.1). ${ }^{105,189 \mathrm{~b}}$ In contrast, Knowles and Dobson et al. found CR has no effect on A $\beta$ fibrillization. ${ }^{246}$ It has been suggested that CR binding arises from a specific conformation of the sulfonate groups in the compound which bind $A \beta$ via electrostatic interactions. ${ }^{242 \mathrm{~d}}$ Other sulfonated dyes investigated as $A \beta$ aggregation inhibitors include chrysamine $G$ and thioflavin $\mathrm{S}^{242 \mathrm{~d}}$ Methylene blue inhibits $\mathrm{A} \beta$ oligomerization by stimulating fibrillization. ${ }^{247}$ It has low toxicity and is able to cross the BBB. Wong and coworkers have studied a series of Brilliant blue derivatives, which are food dyes with blood-brain barrier permeability properties. ${ }^{189 \mathrm{c}}$ Reduction in $\mathrm{A} \beta$-induced cytotoxicity due to the formation of off-pathway non-toxic aggregates was noted for some derivatives.

Necula and coworkers investigated a large series of compounds including many dye molecules and classified them according to whether they selectively inhibit the fibrillization or oligomerization of $A \beta 42$, or both. ${ }^{216}$ The existence of these different classes of inhibitors might suggest that the pathways of $A \beta$ oligomerization and fibrillization are independent. However, as discussed in section 2.6, whether 
oligomers are on- or off-pathway intermediates is still an open question. ${ }^{248}$ In a similar analysis of a smaller number of aromatic compounds, including dyes and polyphenols, Ladiwala et al. ${ }^{249}$ classified the molecules according to whether they (i) remodel soluble oligomers into large non-toxic off-pathway aggregates (some also remodel fibrils), (ii) convert soluble $\mathrm{A} \beta$ oligomers into fibrils but are inactive against fibrillar $A \beta$ or (iii) disaggregate soluble oligomers or fibrils into non-toxic lowmolecular weight species (Fig.14).

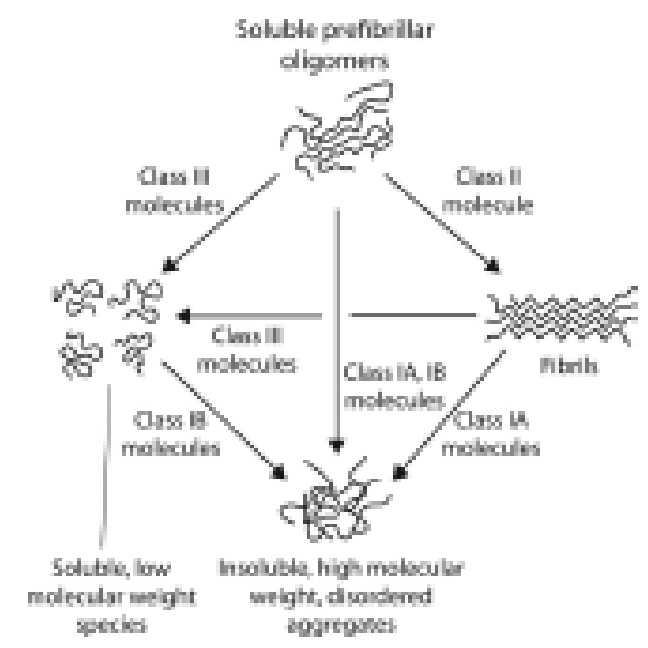

Fig.14. Pathways of aggregation for different classes of small molecule inhibitors of A $\beta$ oligomerization/fibrillization. ${ }^{249}$ This research was originally published in Ladiwala, A.R.A. et al., Journal of Biological Chemistry 2011, 286, 3209. (C) The American Society for Biochemistry and Molecular Biology.

Polyphenols are also able to influence $A \beta$ aggregation. A review provides details of the many compounds investigated in this context $\mathrm{t}^{250}$ and another review describes the associated mechanisms of action with a focus on the antioxidant role of these compounds. ${ }^{251}$ Polyphenols that have attracted particular attention such as tannic acid and epigallocatechin gallate (EGCG) have been shown to reduce $\mathrm{A} \beta$ cytotoxicity in 
cell-based assays, ${ }^{242 a, 242 \mathrm{~d}, 249,252}$ and can disassemble mature $A \beta 42$ fibrils ${ }^{252 b}$ although these compounds are not able to cross the BBB. ${ }^{189 \mathrm{c}}$ Catechins and related polyphenols have also been shown to inhibit A $\beta$ fibrillization, as assayed using ThT fluorescence. ${ }^{242 a, 253}$ It has been noted that competitive binding of the analyte and ThT means that these results need to be treated with caution. ${ }^{254}$ Other biologically derived polyphenols including NDGA (nordihydroguaiaretic acid), curcumin and rosmaric acid have also been examined as $A \beta$ aggregation inhibitors, or in terms of disaggregation of pre-formed fibrils. ${ }^{242 a, 242 \mathrm{~d}}$ Research has developed to the stage of in vivo studies using $\mathrm{Tg} 2576$ mice, with a focus on the pathway of $\mathrm{A} \beta$ aggregation, i.e. via oligomers or otherwise. ${ }^{255}$ Curcumin can cross the BBB and has been shown to reduce plaque burden using a Tg2576 mouse model. ${ }^{256}$ Resveratrol, a polyphenol with antioxidant properties found in wine has been shown to inhibit $A \beta 42$ fibril formation and to reduce cytotoxicity. ${ }^{257}$

Gazit and coworkers have screened a series of small molecule inhibitors of $A \beta$ aggregation that contain aromatic recognition elements as well as $\beta$-breaker units. ${ }^{258}$ A lead compound $\mathrm{NH}_{2}$-D-Trp-Aib-OH was identified. This compound is able to inhibit the formation of toxic oligomers and in vivo studies using a model mouse (expressing human APP Swedish and London mutations) also indicated improved cognitive function. ${ }^{258}$

Metal ion chelators, in particular of $\mathrm{Cu}^{2+}$ and $\mathrm{Zn}^{2+}$, have also been shown to inhibit or reverse aggregation of $\mathrm{A} \beta$ in vitro. ${ }^{242 \mathrm{a}, 242 \mathrm{~d}, 259}$ Cherny et al. indicate that to efficiently extract $\mathrm{A} \beta$ from brain tissue using metal ion chelators including EGTA and ethylene diamine, $\mathrm{Ca}^{2+}$ or $\mathrm{Mg}^{2+}$ are also required. ${ }^{259 \mathrm{a}}$ Metal ion chelators can be specific to 
particular ions, for example clioquinol (Fig.13a), an anti-malerial quinone, selectively binds $\mathrm{Zn}^{2+}$ and $\mathrm{Cu}^{2+}$ with greater affinity than $\mathrm{Ca}^{2+}$ and $\mathrm{Mg}^{2+}$ and reduces brain $\mathrm{A} \beta$ deposition in an Tg2576 mouse model. ${ }^{260}$ Studying A $\beta 40$, Raman et al. found that $\mathrm{Zn}^{2+}$ and $\mathrm{Cu}^{2+}$ but not $\mathrm{Fe}^{3+}$ reduce fibril formation, however pre-formed fibrils are stable in the presence of these metal ions. Clioquinol-induced resumption of fibril growth suppressed by $\mathrm{Cu}^{2+}$ but not $\mathrm{Zn}^{2+}$, points both to a chelation effect but also a synergistic effect of a $\mathrm{Zn}^{2+}$-clioquinol complex on $\mathrm{A} \beta 40$ fibrillization. ${ }^{259 \mathrm{~b}} \mathrm{~A}$ successor to clioquinol is the 8-hydroxyquinoline analogue PBT2 which reached phase II clinical trials, and shows ability to reduced CSF A $\beta 42$ levels as well as cognitive performance. ${ }^{261}$ This compound is believed to perform as a superior ionophore than clioquinol, i.e. to more effectively promote the transport of copper and zinc ions across cell membranes. ${ }^{261 b}$ It is also designed to be easier to synthesize, more soluble and to have increased BBB permeability. ${ }^{261 \mathrm{~b}}$ The role of metal ions in the inhibition of $\mathrm{A} \beta$ fibrillization or promotion is discussed further in Section 4.8. Inspired by clioquinol, bifunctional compounds that can interact with both metal ions and $\mathrm{A} \beta$ have been developed based on pyridine/stilbene derivatives ${ }^{262}$ and related pyridinones. ${ }^{263}$ These compounds can disaggregate $A \beta 40$. A responsive copper chelator that is released when a pro-chelator is cleaved by $\beta$-secretase has been developed and shown to inhibit $\mathrm{Cu}^{2+}$-induced $\mathrm{A} \beta$ aggregation. ${ }^{264}$ Similarly, a pro-drug compound comprising a glycosylated (glucose-receptor targeting) metal ion chelator (hydroxypridinone) is able to cross the $\mathrm{BBB}$ with enzymatic loss of the glucose unit. $^{265}$ The compound has an antioxidant property.

Compounds that can block $\mathrm{A} \beta$-induced channel formation in lipid membranes including tromethamine have been investigated via conductance 
experiments. ${ }^{225 a, 225 b, 266}$ Zinc can also block such channels. ${ }^{229,266 b}$ Arispe et al. also developed a peptide-based channel blocker, designed specifically to modulate late $\mathrm{A} \beta$ effects on caspase activation and apoptosis. ${ }^{266 \mathrm{~b}}$

Glycosaminoglycans or proteoglycans have been associated with AD since sulfated GAGs such as heparan or chondroitin sulfate are present in neuritic plaques, neurofibrillary tangles and vascular amyloid deposits. ${ }^{242 \mathrm{~d}, 267}$ Binding of some sulfated GAGs can prevent the proteolytic degradation of fibrillar A $\beta$. Heparan or heparan sulfate can accelerate the fibrillization of $A \beta$ in vitro, ${ }^{267 \mathrm{~b}}$ probably due to electrostatic binding to a specific domain in the $A \beta(11-28)$ region. ${ }^{245 a}$ Chondroitin sulfate also promotes the aggregation of $A \beta 42$ into stable fibrils of reduced toxicity. ${ }^{268}$ These studies suggested that inhibitors of this interaction might prove useful as therapeutic agents. ${ }^{242 a}$ In a related manner, sulfated compounds such as heparin and dextran sulfate can inhibit the interaction of heparan sulfate with $A \beta .{ }^{269}$ Some sulfated compounds inhibit $\mathrm{A} \beta$ aggregation. Synthetic glycopolymers bearing sulfated saccharide units are also able to suppress the fibrillization of $A \beta 42, A \beta 40$ and $A \beta(25-$ 35). ${ }^{270}$ Simple sugars can either accelerate or inhibit fibrillization. ${ }^{271}$ As mentioned above, the GAG-mimetic tramiprosate (Fig.13b) reached phase III clinical trials but was not successful despite reducing CSF A $\beta 42$ levels in mild-to-moderate AD patients. ${ }^{243 b}$ Reviews that discuss the effect of GAGs on $A \beta$ aggregation are available $^{267 b, 267 c, 272}$ (the former considers also proteins and lipids). The location and distribution of sulfate groups on the GAG chains may define the interaction with $A \beta .^{267 c, 273}$ Fibril formation of $A \beta 42$ is actually promoted in the presence of GAGs with a suitable spacing of sulfate units, although the cationic polysaccharide chitosan can inhibit such aggregation. ${ }^{273}$ Fraser and coworkers showed that in the presence of 
low concentrations of sulfate ions, $A \beta(11-28), A \beta(13-28)$, and $A \beta(11-25)$, but not A $\beta(15-28)$ undergo extensive lateral aggregation into "macrofibers". ${ }^{245 a}$ Ariga et al. point to the key role of the $A \beta(13-16)$ HHQK region in the binding process, especially the histidine residues. ${ }^{274}$ They also note that low molecular-weight heparins can inhibit fibril formation.

As discussed in the Introduction, oxidative stress plays an important role in AD. ${ }^{17}$ The role of antioxidants as novel neuroprotective agents has been reviewed, including polyphenols and other nutraceuticals such as flavonoids and turmeric. ${ }^{235,251}$ The effects of a series of flavonoids, catechins and related compounds on $\mathrm{A} \beta 42$ fibrillization has been compared and cytotoxicity studies were performed on fibril inhibitors (some fibril promotors were also identified) leading to the identification of promising lead compounds. ${ }^{275}$ The structural features of derivatives of the flavonoid fisetin involved in A $\beta 42$ fibril inhibition have been probed. ${ }^{274}$ Several studies have suggested that antioxidant vitamins may reduce neurotoxicity, due to their role in alleviating oxidative stress. ${ }^{242 a}$

Lipid-based small molecule inhibitors have also been developed, since the presence of lipid membranes may accelerate $A \beta$ fibrillization, ${ }^{267 \mathrm{c}}$ since $A \beta$ deposition is initiated in a plasma membrane-bound form (Section 4.7). A particular focus has been on the interaction of $\mathrm{A} \beta$ with phosphatidylinositol since this causes a dramatic increase in fibril growth. This can be inhibited using headgroups from other members of the phosphatidylinositol family. ${ }^{276}$ This led to the identification of scyllo-inositol (cyclohexanehexol, Fig.13c) as lead compound. ${ }^{277}$ This compound stabilizes an oligomeric form of $A \beta{ }^{277}$ Administration to TgCRND8 mice prevented $A \beta$ plaque formation, 
synaptic toxicity and cognitive deficits. ${ }^{84 \mathrm{~b}}$ These effects were seen both in prophylactic and treatment studies. ${ }^{278}$ The compound has high availability due to the presence of the inositol transporters at the BBB. This compound has reached phase II trials (as AZD-103, Transition Therapeutics). ${ }^{279}$

The action of other compounds on $A \beta$ aggregation including nicotine, melatonin, rifampicin and tetracyclines has been reviewed. ${ }^{242 a}$

Inhibition of $A \beta$ aggregation has been targeted via use of self-recognition elements (SREs). These are molecules based on fragments of the A $\beta$ peptide, which are capable of binding to the corresponding sequence in the native peptide, but are modified so as to disrupt $\beta$-sheet fibrillization. ${ }^{242 \mathrm{~d}}$ Findeis et al. proved that compounds based on a core sequence of the $A \beta$ peptide implicated in fibrillization, $A \beta(16-20)$ (KLVFF), showed promise as SREs. ${ }^{280}$ Murphy and co-workers have investigated the effect on $\mathrm{A} \beta$ aggregation of compounds based on KLVFF extended at the C-terminus by cationic or anionic residues to give, for example, KLVFFKKKKKK or KLVFFEEEE. ${ }^{281}$ Molecules containing three or more lysines in the extension were found to be most effective. ${ }^{281-282}$ Modified versions of these compounds have been used to examine the role of surface tension on the kinetics of aggregation of $A \beta 40 .^{283}$ The compounds contained modifications including charge, branching, D/L-isomer substitution and counterion type (motivated by the Hofmeister series) that influence the stabilisation of the protein structure. Gordon and co-workers studied the $N$ methylated compound $A \beta(16-20) \mathrm{m}$ and showed that it inhibits fibrillization of $\mathrm{A} \beta(1-$ 40). ${ }^{284} N$-methylation of alternate residues disrupts $\beta$-sheet self-assembly due to the presentation on one face of the $\beta$-strand of residues incapable of adopting the usual 
hydrogen bonding pattern. ${ }^{284}$ Doig and co-workers have followed this up and screened a number of $N$-methylated "meptides" based originally on the same sequence, but with all D-amino acids and with modifications of the termini and incorporation of branched residues in certain positions, that are promising as SREs towards targeted aggregation inhibitors, and which also contain elements to improve their pharmacokinetics. ${ }^{242 c, 285}$ A lead compound based on a pentapeptide framework has been shown to have favourable cytotoxicity, and to reverse the inhibition of LTP by $\mathrm{A} \beta 40 .{ }^{242 \mathrm{c}}$ Doig et al. have also reviewed other promising compounds. ${ }^{242 \mathrm{~b}, 242 \mathrm{c}}$ Austen et al. have developed compounds based on KLVFF but with terminal modifications to aid solubility and showed that these were effective inhibitors of toxicity using human neuroblastoma cells. ${ }^{286}$ The binding of $\beta$-alanine and GABA-modified peptide fragments to amyloid fibrils formed by $\alpha$-synuclein, $A \beta 40$ and amylin has recently been examined. ${ }^{287}$ The binding sequence was based on self-recognition element $\mathrm{V}^{77} \mathrm{AQKTV} \mathrm{V}^{82}$ of the full length $\alpha$-synuclein peptide and shorter sequences therefrom. Pentapeptides containing gamma-amino acids and the KLVFF SRE (or its D-amino acid variant) have also been examined, and a promising compound able to reduce $A \beta$ toxicity, due to inhibition of fibrillization, was identified. ${ }^{288}$ Watanabe and coworkers have shown by a binding assay using immobilized KLVFF in the presence of fluorescently-labelled KLVFF in solution that the KLVFF motif is a self-recognition element. ${ }^{289}$ Incorporation of the retro-inverse peptide ffvlk into cross-linked PEG networks (as a monomer, dimer or tetramer, all linked to PEG via a cysteine residue) produces gels that can bind $\mathrm{A} \beta 42 .{ }^{290}$ It was proposed that these gels could serve as sinks or "detoxification depots" to capture $A \beta$. 
Ligands that stabilize the $A \beta(13-26)$ domain in an $\alpha$-helical conformation (as revealed by NMR, section 4.1) have been studied as a means to reduce aggregation of $A \beta 40$ and $\mathrm{A} \beta 42$, and also cytotoxicity. ${ }^{109 \mathrm{e}}$ The inhibitors were based on self-recognition modelling of the $\mathrm{A} \beta(13-23)$ region and comprise two peptidomimics and $N$-decanoyldiethyelenetriamine. Studies using a Drosophila melanogaster model (expressing human $A \beta 42$ ) also suggested reduced neurodegeneration in the presence of the molecules. ${ }^{109 e}$ Schrader and coworkers have explored the use of functionalized aminopyrazole derivatives in binding to $A \beta 42 .{ }^{291}$ Ligands bearing multiple lysine residues were found to interact with the ladder of stacked E22 residues and to completely dissolve pre-existing fibrils. ${ }^{291 \mathrm{~b}}$ These ligands interact through electrostatic and hydrophobic interactions with the KLVFF sequence although lipophilic groups on some of the designed ligands can interact with the nonpolar residues between I31 and V36. ${ }^{291}$ This group have also developed lysine-specific molecular tweezers (Fig.15) with aromatic pincers able to inhibit the aggregation and toxicity of $\mathrm{A} \beta 40$ and $\mathrm{A} \beta 42 .{ }^{292}$ Mihara et al. have shown that even dipeptides LF and CF can form mixed fibrils with small amounts of $A \beta 42$, hence "capturing" the peptide. $^{293}$
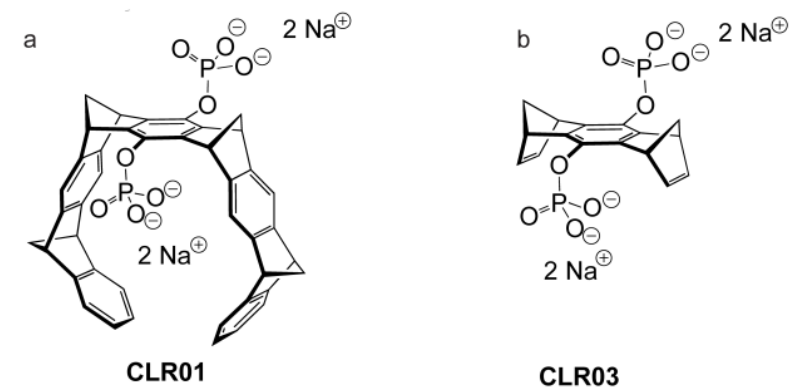

Fig.15. Lysine-specific molecular tweezers CLR01 and CLR03. Reprinted with permission from ref. ${ }^{292}$. Copyright 2011 American Chemical Society. 
Statins reduce cholesterol levels in blood and this in turn correlates to $\mathrm{A} \beta$ production and aggregation as discussed in Section 4.7. Reduction in cholesterol using lovastatin and methyl- $\beta$-cyclodextrin inhibits the production of $A \beta$ in hippocampal neurons without altering APP production. ${ }^{294}$

In a novel approach related to the "toxic oligomer" hypothesis (Section 2.6.1), the action of selective fibrillization accelerators (with an oligomer-reduction activity) has been probed. ${ }^{191}$ Compounds related to the dye orcein are shown to accelerate $A \beta 42$ fibril growth, while reducing oligomer formation.

\subsubsection{Proteins and Particles}

Chaperone proteins have been shown to bind to $A \beta$ and to inhibit fibrillization/ oligomerisation. Several proteins that interact with intracellular $A \beta$ in vivo have been identified based on mass spectrometry analysis of human $\mathrm{A} \beta$ expressed in C. elegans. including the small heat-shock protein $\alpha \mathrm{B}$-crystallin and related proteins such as members of the HSP70 family. ${ }^{295} \alpha \mathrm{B}$-crystallin is a chaperone for $\mathrm{A} \beta$, binding to it, and preventing fibril growth. ${ }^{296}$ It prevents the spontaneous fibrillization of $A \beta 42^{296}$ and the $A \beta 42$-seeded growth of $A \beta 40 .{ }^{296 a}$ The extracellular chaperone protein clusterin has been shown to sequester oligomers of $A \beta 40$ during aggregation and disaggregation. ${ }^{101}$ As discussed above, clusterin (apolipoprotein $\mathrm{J}$ ) is an identified risk factor in Alzheimer's disease. ${ }^{54,64}$ A large-scale screening study has recently identified small molecule proteostasis regulators that induce expression of the chaperone heat shock transcription factor-1 (HSF-1). ${ }^{297}$ This may form the basis to 
treat a number of protein conformational diseases including AD. The inhibition of fibrillization in the presence of clusterin (Section 2.1.1) has also been examined. ${ }^{298}$

Other proteins and peptides have been shown to bind $A \beta$ and to influence its aggregation. The secretory protein gelsolin ${ }^{299}$ and the ganglioside $\mathrm{GM} 1^{300}$ are able to bind $\mathrm{A} \beta$, and it has been proposed that peripheral treatment with these materials can be used to reduce $A \beta$ levels in brain (mouse model). ${ }^{301}$ Transthyretin, itself an amyloidogenic protein, and variants, can inhibit the aggregation of $\mathrm{A} \beta$ in vitro and in vivo. ${ }^{302}$ The cellular prion protein $\operatorname{PrP}^{\mathrm{C}}$ is also able to bind $\mathrm{A} \beta$ oligomers at nanomolar affinity, indicating that it may act as a receptor and that it is involved in A $\beta$-induced synaptic disfunction in the mouse brain. ${ }^{303}$ However, these findings have not been replicated by two other groups who found that mice suffered memory deficits even in the absence of prion protein, carefully excluding $\operatorname{PrP}^{\mathrm{C}} \cdot{ }^{146 e, 146 \mathrm{f}}$ Very recent work suggests that cellular $\operatorname{PrP}^{\mathrm{C}}$ is essential for oligomeric $\mathrm{A} \beta$-induced cell death, since PrPC antibody blocks A $\beta$ oligomer-induced neurotoxicity and mice expressing PrPC are resistant to $\mathrm{A} \beta$ toxicity. ${ }^{304}$ It has been proposed that $\operatorname{PrP}^{\mathrm{C}}$ may have a physiological role in modulating NMPAR activity, mediated by copper ions, which is disrupted in the presence of $\mathrm{A} \beta 42$ (section 4.8). ${ }^{305}$

Polymeric nanoparticles (uncharged acrylamide-based copolymers) inhibit the fibrillization of $A \beta 40$, an observation ascribed to the binding of $A \beta$ (in monomeric or oligomeric form) to the nanoparticles. ${ }^{114}$ The binding mainly affects nucleation, and the lag time was found to be strongly influenced by the copolymer composition. The binding is due to a combination of hydrophobicity (controlled via copolymer composition) and hydrogen bonding between polar groups on the polymer and in 
$\mathrm{A} \beta .{ }^{114}$ In the case of cationically (amide) functionalized polystyrene nanoparticles, inhibition of fibrillization is observed for high particle surface area, whereas fibrillization is accelerated for low particle surface areas due to reduction of the lag phase. $^{306}$

Inorganic nanoparticles can function as $\mathrm{A} \beta$ fibrillization inhibitors, ${ }^{307}$ although this was demonstrated with cytotoxic CdTe nanoparticles. The precise mechanism is unclear. Polyoxometalates which comprise inorganic early transition metal clusters also inhibit the aggregation of $A \beta .{ }^{308}$ Surprisingly, organic nanoparticles based on porous silica have been shown to penetrate the brains of fruit flies (D. melanogaster), without exhibiting neurotoxic effects and potentially enabling delivery across the BBB ${ }^{309} \mathrm{BBB}$ permeability can be modeled using the parallel artificial membrane permeability assay, which measures passive diffusion of small molecule through an artificial lipid membrane. $^{262,310}$

One example of a study using dendrimer molecules employed maltose-functionalized dendrimers to influence the fibrillization of $\mathrm{A} \beta 40 .{ }^{311} \mathrm{~A}$ smaller dendrimer led to fibrillar clumps, sequestering the $\mathrm{A} \beta$ and reducing toxicity, whereas a larger one produced amorphous aggregates, toxic to cells.

\subsection{Inhibitors of the secretase enzymes}

It has proved difficult to identify small molecule inhibitors of $\beta$-secretase (BACE1)

with favorable pharmacokinetic characteristics. ${ }^{242 a}$ The crystal structure of $\beta$-secretase complexed to an 8-residue peptide inhibitor EVNLAAEF has been reported - the enzyme has the usual binding cleft of an aspartyl protease. ${ }^{312}$ A Japanese group have 
developed several peptide-based compounds based on phenylnorstatine. ${ }^{313}$ A lead compound KMI-429 $9^{313 b}$ was explored further in vivo using a mouse model. ${ }^{314}$ Since the enzyme is membrane bound, an inhibitor has recently been developed by linking a peptide $\beta$-secretase inhibitor to a sterol moiety designed to insert in the membrane. ${ }^{315}$ Statins may be used to inhibit cholesterol biosynthesis and the expression of BACE and ultimately $A \beta$ production. ${ }^{316}$ Statins can inhibit the dimerization of BACE by inhibiting lipidation. ${ }^{317}$ This may provide a therapeutic target to reduce $A \beta$ production. The role of protein-protein interactions in the correct assembly of BACE, which is required for $\mathrm{A} \beta$ production has been discussed. ${ }^{317} \mathrm{BACE}$ associates with APP in cholesterol-rich rafts. ${ }^{318}$ Knocking out the $\beta$-secretase gene BACE1 causes no adverse phenotype in mice, ${ }^{319}$ indicating that $\beta$-secretase inhibitors may still provide an attractive target for treatments.

A greater number of compounds have been developed as potential inhibitors of $\gamma$ secretase. A complicating factor here is that NOTCH1 and other ligands are also $\gamma$ secretase substrates (as well as APP) ${ }^{242 a}$ and therefore Notch-related side effects may be problematic. ${ }^{320}$ However, certain non-steroidal anti-inflammatory drugs (NSAIDs) can modulate $\gamma$-secretase cleavage without blocking Notch cleavage. ${ }^{321}$ Some NSAIDs can reduce $A \beta$ levels independent of cyclooxygenase (COX enzyme, associated with inflammation) activity. ${ }^{321 \mathrm{a}-\mathrm{c}}$ Application of NSAIDs including ibuprofen, flurbiprofen and indomethicin inhibits the release of amyloidogenic $A \beta 42$ from cultured cells. ${ }^{321 a}$ Flurbiprofen (Fig.13d, in the $R$ enantiomeric form, shown to be particularly effective ${ }^{322}$ ) reached phase III clinical trials, but these were unsuccessful. The target of $\gamma$-secretase modulators, such as flurbiprofen and related 
compounds, has been located to $\mathrm{A} \beta(28-36)$ based on investigation of the localization of fluorescently labeled and biotinylated variants of these NSAIDs. ${ }^{321 \mathrm{e}}$ Some $\gamma$ secretase modulators were shown to alter the production of cell-derived $\mathrm{A} \beta$ oligomers $^{321 \mathrm{e}}$ while compounds that interact with this region of $\mathrm{A} \beta$ act as $\gamma$-secretase inhibitors, highlighting the interplay between $\gamma$-secretase-influenced A $\beta$ production and $A \beta$ aggregation. ${ }^{321 e}$ Furthermore, since some NSAIDs bind to to an APP substrate rather than $\gamma$-secretase, Notch toxicity may be avoided in this way. ${ }^{321 e}$

The $\gamma$-secretase inhibitor Semagacestat (Fig.13e) was shown to reduce formation of $\mathrm{A} \beta$ in cell assays and also in vivo studies. ${ }^{205,323}$ Lanz et al. show that this compound leads to lowering of $A \beta$ in plasma (in guinea pigs) at low doses, but an elevation of A $\beta$ levels at low concentrations. ${ }^{323}$ However, Semagacestat was not successful (due to inferior performance relative to placebo) in phase III clinical trials.

Other strategies have been employed to avoid side effects from Notch impairment. Other than NSAIDs, $\gamma$-secretase inhibitors that block $A \beta$ production without influencing Notch activity such as the thiophene sulfonamide Begacestat (Fig.13f) have reached clinical trials, although the mechanisms are unclear. ${ }^{324}$ Serneels et al. have targeted specific components of the $\gamma$-secretase complex (Section 2.2.3), specifically produced by two $A P H I(A P H I A$ and $A P H 1 B)$ genes. ${ }^{325}$ Inactivation of the Alph1B complex decreases A $\beta$ plaque deposition and improves behavioural deficits. The different Alph1 complexes also produce $A \beta$ of different length. ${ }^{325}$ As an alternative to targeting $\gamma$-secretase, the reduction of $\gamma$-secretase-activating protein (GSAP) has been shown to decrease $\mathrm{A} \beta$ production in cells and in vivo using a mouse 
model. ${ }^{326}$ The anticancer drug imatinib (Gleevec, Novartis) is able to inhibit A $\beta$ production without affecting Notch cleavage since it prevents interaction of GSAP with the $\gamma$-secretase substrate. Thus, GSAP is a potential selective therapeutic target avoiding side effects associated with $\gamma$-secretase inhibition. ${ }^{326}$ Phiel et al. showed that therapeutically relevant doses of lithium chloride, a GSK-3 inhibitor, block the production of $\mathrm{A} \beta$ peptides by interfering with APP cleavage at the $\gamma$-secretase step, but do not inhibit Notch processing. ${ }^{46}$

\subsection{Immunization}

In the development of $A \beta$-based immunotherapies, several strategies have been pursued including passive immunization with monoclonal anti-A $\beta$ antibodies, active immunization with synthetic $A \beta 42$ and active immunization with modified $A \beta$ fragments (Fig.16). ${ }^{327}$ Progress with active immunization has been dogged by the failure of phase IIa clinical trials due to the development of meningoencephalitis in several patients (vide infra).

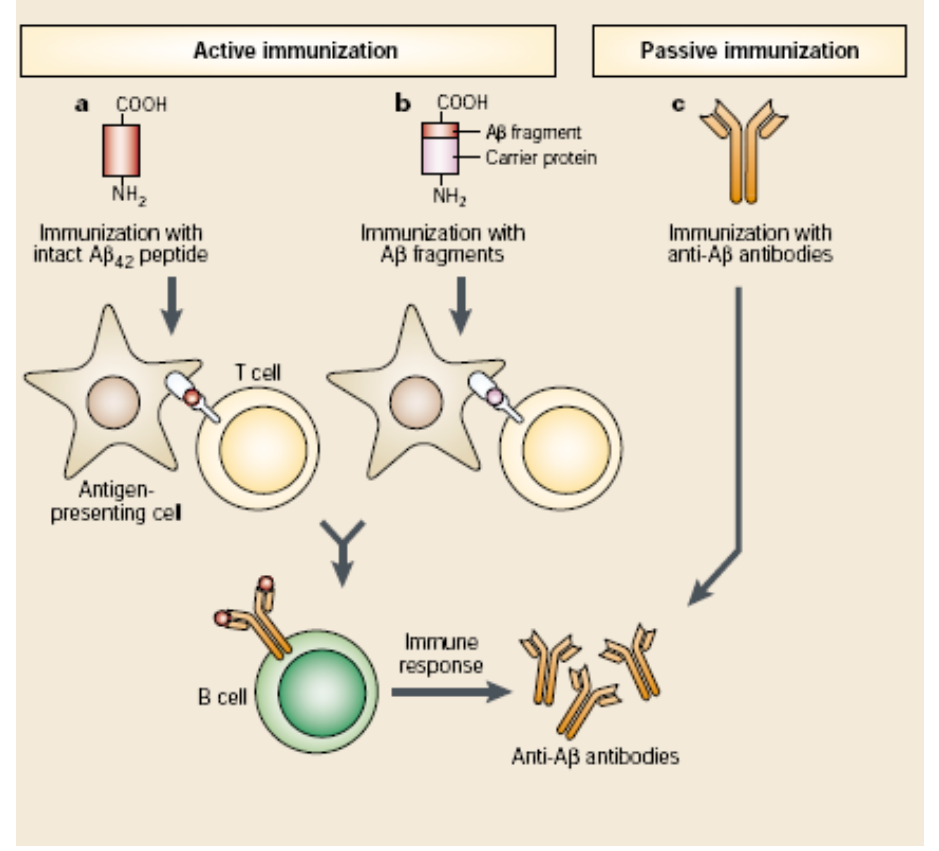


Fig.16. Antibody strategies. ${ }^{327 a}$ Three different approaches are illustrated. The first (panel a) involves immunization with full-length $A \beta 42$. After injection, the peptide is taken up by antigen-presenting cells, and fragments of the peptide are presented to $\mathrm{T}$ cells. Subsequently, various B cells that recognize epitopes on A $\beta 42$ are engaged and proliferate. These eventually produce anti-A $\beta$ antibodies. The second active immunization method (panel b) involves administration of small fragments of $A \beta$ conjugated to an unrelated carrier protein. This approach is similar to the first with the exception that the $T$ cells are stimulated by the carrier protein rather than the $A \beta$ fragment (which lacks T cell epitopes). This approach yields a strong antibody response to part of the $\mathrm{A} \beta$ peptide. The third strategy (panel c) is to administer anti$\mathrm{A} \beta$ antibodies directly. This does not require any immunological response from the host and might be useful in individuals in which an immune response is not otherwise produced. Reprinted by permission from Macmillan Publishers Ltd: Nature Reviews Neuroscience 3, 824, copyright 2002.

Active immunization of transgenic mice with fibrillar $A \beta 42$ leads to a reduction in $A \beta$ deposits in the brain (mouse model) and prevents development of plaque pathology when administered before its formation. ${ }^{83 c, 328}$ The $A \beta$ antibodies generated were reported to reduced $A \beta$ plaque deposition without reducing overall $A \beta$ levels. ${ }^{84 a, 329}$ An improvement in the cognitive performance of mice was also noted. ${ }^{84 a, 329}$ Phase IIa trials pursued by Elan pharmaceuticals, based on this approach (using aggregated A 342 termed AN1792 with an immunogenic adjuvant QS-21) were halted when 6\% of patients developed meningoencephalitis. ${ }^{327 a, 328,330}$ However, after one year patients producing antibodies that targeted plaques had a lower rate of cognitive decline. ${ }^{328}$ In 
further follow-up studies it was found that there was a reduction in CSF tau levels following $A \beta 42$ immunization. ${ }^{331}$ and a decrease in brain volume. ${ }^{332}$ Although clearance of $A \beta$ plaques occurred, this did not prevent neurodegeneration. ${ }^{333}$ Using a triply transgenic mouse $(3 \times \mathrm{Tg})$ model it has been shown that $\mathrm{A} \beta$ immunotherapy can not only reduce $A \beta$ plaques, but also early hyperphosphorylated tau pathology. ${ }^{334}$ Vaccination with soluble oligomers of $A \beta 42$ has also been proposed as a method to produce toxicity-reducing antibodies. ${ }^{204}$ Intraperitoneal injection of $A \beta$-laden brain extracts into the brains of transgenic mice leads to the deposition of amyloid plaques after prolonged incubation time. ${ }^{335}$ These observations point to the possible prion-like behaviour of $A \beta$ and the need for great caution (at the very least) in the development of immunization using $A \beta$ directly.

Sigurdsson et al. ${ }^{336}$ investigated immunization using the modified peptide $\mathrm{K}_{6} \mathrm{~A} \beta(1$ 30)- $\mathrm{NH}_{2}$, with an $\mathrm{N}$-terminal hexa-lysine extension to $\mathrm{A} \beta(1-30)$, the oligo(lysine) enhancing immunogenicity, this extension following work by Palitto et al. ${ }^{282}$ (section 3.2.1). Using a transgenic APP mouse model, this peptide was found to reduce the burden of hippocampal and cortical $\mathrm{A} \beta$, and more particularly the amount of soluble A $\beta 42$ in brain tissue. ${ }^{336}$

Passive immunization using antibodies prevents amyloid formation by brain extract from AD patients or APP23 transgenic mice (which over-express A $\beta 40$ which deposits in diffuse and filamentous form), pointing to the suppression of seeding activity. ${ }^{89}$ It has been suggested that $\mathrm{A} \beta$ disrupts the integrity of the $\mathrm{BBB}$ and that this is restored after immunization as the immune system clears $A \beta$ from the brain. ${ }^{337}$ The 
ability of $A \beta 40$ to cross the $\mathrm{BBB}$ and increase its permeability had been observed earlier, using a cell culture model. ${ }^{338}$

Passive immunization using the humanized monoclonal antibody bapineuzumab against $\mathrm{A} \beta$ got as far as phase II clinical trials. ${ }^{339}$ However, no significant benefit was observed in cognitive performance tests, and in addition some patients exhibited vasogenic edema. ${ }^{339 a}$ However, this compound continues to be investigated in ongoing phase III trials. ${ }^{14 \mathrm{~b}, 279 \mathrm{a}, 340}$ as is the related compound solaneuzumab. ${ }^{14 \mathrm{~b}, 279 \mathrm{a}, 341}$ Passive immunization using monoclonal antibodies (mAbs) has been investigated by Solomon et al. ${ }^{190 \mathrm{~b}, 342}$ They found via in vitro studies that selected mAbs prevent the aggregation of $A \beta$, antibodies recognizing epitopes within $A \beta(1-28)$ and $A \beta(8-17) .{ }^{342}$ Antibodies raised against $A \beta(1-28)$ can disaggregate $A \beta$ fibrils in vitro and reduce the neurotoxic effects of A $\beta$ in vivo (MTT cytotoxicity assays using PC12 cells). ${ }^{190 \mathrm{~b}}$ Antibodies selectively directed against residues 4-10 of $A \beta 42$, inhibit both $A \beta$ fibrillogenesis and cytotoxicity without eliciting an inflammatory response, in addition they can disaggregate preformed A $\beta 42$ fibrils. ${ }^{195}$ De Mattos et al. showed that an antibody (m266, Table 3) against $A \beta 40$ is able to bind and completely sequester plasma $A \beta .{ }^{188 a}$ Peripheral administration leads to a large and rapid increase in plasma $A \beta$ due to a change in $A \beta$ equilibrium in plasma and in CNS. The m266 antibody does not bind to $\mathrm{A} \beta$ deposits in the brain. ${ }^{188 \mathrm{a}}$ However, using mouse anti-A $\beta$ $\mathrm{IgG}_{1}$ antibodies (recognizing $\mathrm{A} \beta(1-16)$ ) some clearance of compact amyloid deposits is observed after several days, along with microglial activation. ${ }^{86}$ The increase in plasma $A \beta$ levels was correlated to $A \beta$ load in the hippocampus and cortex (of PDAPP mice) after immunization. ${ }^{103}$ Administration of m266 to PDAPP can improve 
cognitive learning, despite no alteration in brain A $\beta$ burden. ${ }^{343}$ A complex of m266 and $\mathrm{A} \beta$ was found in the plasma and CSF of treated mice. Bard et al. ${ }^{344}$ noted the presence of anti-A $\beta$ antibodies in the central nervous system after peripheral administration, which were able to bind $A \beta$ plaques and to reduce the pre-existing $A \beta$ burden. The mechanisms of clearance of $\mathrm{A} \beta$ in vivo by immunotherapy have been investigated, this showed that direct disruption of plaques as well as Fc-dependent phagocytosis was involved. ${ }^{345}$

Immunization using antibodies to human serum amyloid $\mathrm{P}$ component leads to the elimination of visceral amyloid desposits, in mice. ${ }^{346}$ The plasma glycoprotein human serum amyloid P (SAP) component non-selectively binds all kinds of amyloid deposits. ${ }^{347}$ Clinically, it is possible to reduce circulating human SAP using a known SAP-depleting compound, thereby stimulating antibody production. Inhibitors of SAP binding to $\mathrm{A} \beta$ fibrils have also been developed, and the lead compound (CPHPC) also reduced levels of circulating human SAP. ${ }^{346-348}$ This technology is proceeding to human clinical trials. ${ }^{349}$

\subsection{Other Approaches}

Other treatment strategies are being pursued, these are discussed elsewhere ${ }^{8,12 a, 31}$ and are not considered further here as they do not involve A $\beta$. Summaries of compounds in phase I, II and III clinical trials are available. ${ }^{14,235,279}$

Due to a correlation between insulin resistance (section 2.1) and $\mathrm{AD}$, the peroxisome proliferator-activated receptor- $\gamma$ (PPAR- $\gamma$ ) agonist rosiglitazone has been investigated, ${ }^{51}$ and has reached phase III trials. ${ }^{31}$ This compound is an insulin sensitizer and 
mitochondrial activator, and it activates the PPAR- $\gamma$ pathway. This increases dendritic spin density and rescues spine loss caused by APOE- $\varepsilon 4 .{ }^{51}$ Nerve Growth Factor (NGF) mimics have also reached phase III trials, ${ }^{31}$ however since this does not involve $A \beta$ it is outside the scope of the present review. An antihistamine drug Dimebon $^{350}$ reached phase III clinical trials for AD which failed due to the absence of a significant effect. ${ }^{351}$ The clearance of $A \beta$ is facilitated by ApoE (section 2.1.1). This process is impaired in $\mathrm{AD}$. The compound bexarotene that influences ApoE expression has been shown to enhance clearance of soluble A $\beta$ in an ApoE-dependent manner, also improving cognitive performance in mice. ${ }^{352}$

\section{BIOPHYSICAL CHEMISTRY STUDIES OF A $\beta$ AND FRAGMENT PEPTIDES-STRUCTURE AND AGGREGATION}

\subsection{A $\beta$ Conformation and Structure of Fibrils}

Amyloid fibrils contain bundles of $\beta$-sheets with backbones orthogonal to the fibre axis, in the so-called "cross- $\beta$ " structure (Fig.17), and this is observed in fibre X-ray diffraction (XRD) patterns obtained from stalks of $A \beta$ and fragments. ${ }^{27 a, 27 b, 27 d, 27 e, 353}$ The prominent equatorial reflections in Fig.17a (a typical amyloid fibre XRD pattern)

arise from the spacing of stacked $\beta$-sheets (10-12 $\AA$, the range of values arising from different side chain packing modes) and the meridional reflections arise from the $\beta$ strand spacing (4.7-4.8 $\AA$ ).
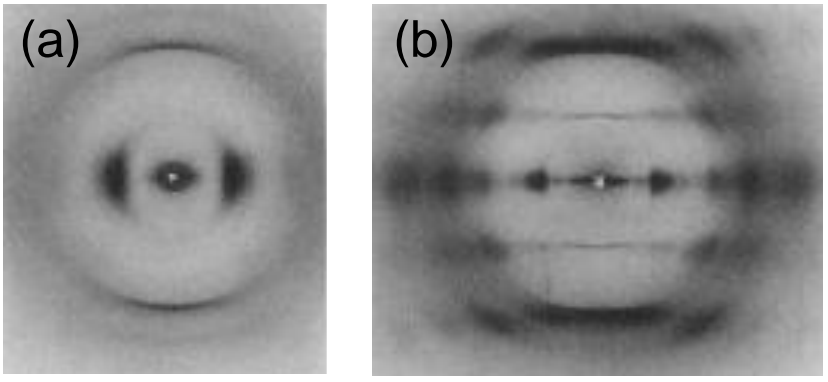
Fig.17. Fibre X-ray diffraction patterns from (a) $A \beta 40$, (b) $A \beta(11-28) .{ }^{353 a}$ Reprinted from Inouye, H. et al. Biophysical Journal 1993, 64, 502, Copyright 1993, with permission from Elsevier

Many studies have analysed the conformational tendencies of $\mathrm{A} \beta$ and associated fragments. Early work is reviewed by Serpell ${ }^{27 d}$ and others. ${ }^{354}$ A study using CD and NMR showed that $A \beta 40$ in aqueous buffer solution adopts a predominantly polyproline II conformation at $0{ }^{\circ} \mathrm{C}$ and a random coil state at $60{ }^{\circ} \mathrm{C} .{ }^{355}$ The residues in the central hydrophobic region $\mathrm{A} \beta(12-28)$ tend to adopt $\beta$-strand-like conformations at temperatures below $20{ }^{\circ} \mathrm{C}$. The thermal PPII - random coil transition was found to be weakly cooperative. The conformation of the $\mathrm{N}$ terminal $\mathrm{A} \beta(1-9)$ domain was also analysed. ${ }^{355} \mathrm{CD}$ has also been exploited to examine the secondary structure formation of $A \beta(1-28), A \beta(1-39), A \beta 42$ and $A \beta(29-42)$ in water and TFE or HFIP. ${ }^{356}$ In aqueous TFE, $A \beta 42, A \beta(1-28)$ and $A \beta(1-39)$ adopt an $\alpha$-helical structure at low and high $\mathrm{pH}$, but a $\beta$-sheet structure at intermediate $\mathrm{pH} .{ }^{356 \mathrm{a}} \mathrm{A} \beta 42$ and $\mathrm{A} \beta 40$ have a net charge of +3 at $\mathrm{pH} 7.4$ and $\beta$-sheet formation is possible under these conditions. The solubility of $\mathrm{A} \beta 42$ and shorter peptides in aqueous solution was studied as a function of $\mathrm{pH}$ and concentration and aggregation was found to be enhanced at low $\mathrm{pH}^{90 \mathrm{a}}$ Murphy and coworkers investigated the aggregation of $\mathrm{A} \beta(1-$ 28), including the kinetics of aggregation via light scattering. ${ }^{357}$ 
Much effort has focussed on analysing the conformation of the core hydrophobic domain of $A \beta$. NMR provides the most detailed conformational information. Benzinger et al. applied ${ }^{13} \mathrm{C}$ cross-polarization magic angle spinning solid state NMR to probe the conformation of $\mathrm{A} \beta(10-35) .{ }^{358}$ Multiple quantum analysis using the DRAWS pulse sequence enables the registry of specific labelled residues to be determined. This revealed a parallel $\beta$-sheet structure with residues in register. The parallel in-register structure persists despite $\mathrm{pH}$ dependent variation in fibril morphology as revealed by electron microscopy. ${ }^{359}$ Similar solid state NMR techniques (with constraints from X-ray diffraction and TEM measurements) have indicated a parallel configuration of $\beta$-strands of $A \beta 40$, each molecule of which has a $\beta$-strand/turn/ $\beta$-strand arrangement (Fig.18). ${ }^{360}$ A similar parallel in-register arrangement was deduced from solid-state NMR data for $\mathrm{A} \beta 42 .{ }^{359}$ A turn structure was located in the $A \beta(26-29)$ domain based on proteolysis of disulfide-bridged $\mathrm{A} \beta(10-43)$ analogues. ${ }^{361}$ A turn was also predicted around residue 26 , based on a primary sequence conformation index. ${ }^{362}$ This feature was also anticipated based on MD simulations of $A \beta(16-35)$ and $A \beta(10-35)$ that showed turns in the $A \beta(24-27)$ region due to intramolecular D23-K28 salt bridging. ${ }^{363}$ In contrast to A $\beta 40$ and A $\beta 42$ which show in-register parallel $\beta$-sheets, NMR studies reveal that Iowa mutant D23N A $\beta 40$ can form antiparallel $\beta$-sheets. ${ }^{364}$ 


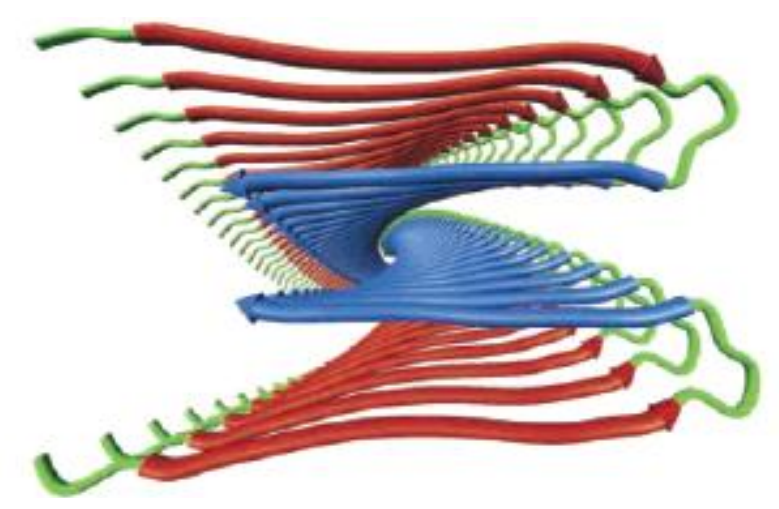

Fig.18. Stacking of $A \beta 40$ into parallel $\beta$-sheets according to modelling with constraints from solid state NMR. Reprinted with permission from ref. ${ }^{360 b, 360 \mathrm{c}}$. Copyright 2011 American Chemical Society.

Detailed conformations of the $\beta$-strand/turn/ $\beta$-strand U-shaped arrangement have been compared for the Ma-Nussinov ${ }^{363}$ models and the NMR derived conformations of Lührs ${ }^{365}$ and Tycko. ${ }^{360 b, 360 c, 366}$ Replica-exchange molecular dynamics (REMD) and discrete molecular dynamics (DMD) simulations ${ }^{367}$ have also confirmed these features, as discussed in Section 4.2. Later MD simulations confirmed a turn in the $\mathrm{A} \beta(23-28)$ domain. ${ }^{368}$ Benzinger et al. did not find evidence for a turn structure in the $\mathrm{A} \beta(25-29)$ domain in their study of $\mathrm{A} \beta(10-35),{ }^{358 \mathrm{~b}}$ highlighting the importance of the E22 and V24 residues in salt bridging and hydrophobic interactions respectively. An analysis of fibre X-ray diffraction data from $A \beta(11-25)$ fragments was also consistent with a hairpin turn structure, but in the L17-F20 domain. ${ }^{353 b}$ However, in a later report from the same group, the unit cell was modelled based on an extended conformation of $A \beta(11-25) .{ }^{369}$ The $\beta$-sheet structure of this peptide has been imaged by cryo-TEM which indicates in-register $\beta$-strands. ${ }^{370}$ 
In contrast to work focussed on the core hydrophobic domain, there have been fewer studies on the conformation of the $\mathrm{C}$ terminal region. The hydrophobic $\mathrm{C}$-terminal $\mathrm{A} \beta(29-42)$ segment forms a $\beta$-sheet structure independent of $\mathrm{pH}$, solvent or temperature ${ }^{356}$ pointing to the high aggregation propensity of this domain, in agreement with computer simulations discussed above. The important role of the A $\beta$ (33-35) domain was also shown by Pike et al. who performed a thorough study on the secondary structure of variants (amino acid deletions and substitutions) of $A \beta(25-$ 35) via $\mathrm{CD}$ and on the neurotoxicity of these peptides. ${ }^{371}$ These authors also imaged fibril morphology using electron microscopy. ${ }^{371}$ Aggregation of $\beta$-sheets is found to be maximal at $\mathrm{pH} 5.4{ }^{356 \mathrm{~b}} \mathrm{~A}$ low resolution NMR study indicated a pleated antiparallel $\beta$-sheet structure for $A \beta(34-42) .{ }^{372}$ Hoyer and coworkers reported, on the basis of NMR and computer modelling, a $\beta$-hairpin structure for $A \beta 40$ in a complex with a phage-display selected affibody protein. ${ }^{200}$ The hairpin comprises residues $\mathrm{A} \beta(17-36)$. On the other hand, SDS at $\mathrm{pH} 7.2$ stabilizes an $\mathrm{A} \beta 42$ conformation comprising an extended chain (D1-G9), two $\alpha$-helices (Y10-V24 and K28- A42), and a looped region (G25-S26-N27) as revealed by solution NMR. ${ }^{373}$ Helical content of $\mathrm{A} \beta(12-28)$ in the K16-V24 domain in presence of SDS is also confirmed by CD. ${ }^{374}$ Figure 19 shows the $\beta$-strand/turn/ $\beta$-strand conformation of $A \beta(18-42)$ (residues 1-17 are disordered) within the cross- $\beta$ fibril structure. 


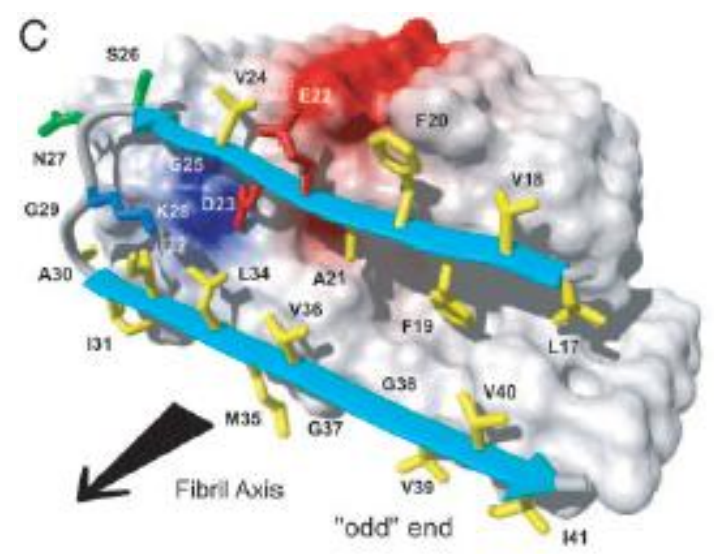

Fig.19. Conformation of $A \beta(17-42)$ as revealed by H/D-exchange NMR. ${ }^{365}$ PDB: 2BEG. The hydrophobic, polar, negatively charged and positively charged amino acids are shown in yellow, green, red and blue respectively. Positively and negatively charged surface patches are shown in blue and red, others in white. Copyright 2005 National Academy of Sciences, USA.

Disctinct from longer $A \beta$ fragments (except the Iowa mutant, discussed above), the $\mathrm{A} \beta(16-22)$ peptide appears to adopt an antiparallel $\beta$-sheet arrangement as indicated by solid state NMR, ${ }^{375}$ and isotope-edited FTIR on labelled peptides (combined with ssNMR) $)^{376}$ and computer simulation. ${ }^{363}$

The crystal structure of several A $\beta$ fragments has recently been reported, specifically $A \beta(16-21)$ (for which three polymorphs were observed), $A \beta(27-32), A \beta(29-34)$, $A \beta(30-35), A \beta(35-42)$ (in two forms), $A \beta(35-40)$ (in two forms) and $A \beta(37-42){ }^{377}$ These peptides all form steric zipper structures, i.e. based on self-complementary pairs of $\beta$-sheets. Sequences prone to form steric zipper structures were screened using a 3D-profile self-association energy calculation, which indicated a cluster of aggregation-prone sequences in the $C$ terminal $A \beta(30-42)$ domain. ${ }^{377}$ In the first 
report by Eisenberg's group on crystal structures of amyloid steric zipper structures A $\beta$ peptides including $A \beta(37-42)$ GGVVIA and $A \beta(35-40)$ MVGGVV were among the peptides studied. ${ }^{378}$ GGVVIA belongs to the class of parallel up-down face-toback $\beta$-sheet structures while MVGGVV $\beta$-sheets adopt an antiparallel up-down faceto back arrangement.

Based on cryo-TEM images, $A \beta 40$ fibrils are reported to comprise two protofibrils whatever the overall fibril morphology (considerable polymorphism was noted, see also section 4.4). ${ }^{379}$ An initial report based on cryo-TEM suggests that $A \beta 42$ forms hollow fibrils in which the hairpin $A \beta(17-42)$ regions fit within the reconstructed density map of the shell. ${ }^{380}$ However, a later report from the same group based also on cryo-TEM along with additional mass-per-length measurements from scanning TEM, indicates that $A \beta 42$ forms a single filament structure without a hollow core (Fig.20). ${ }^{381}$ The cryo-TEM images published for $A \beta(11-25)$ also do not show a hollow interior. ${ }^{370}$ Malinchik et al. had earlier proposed a hollow filament structure for $A \beta 40$ on the basis of TEM cross-section images on plastic-embedded samples, as well as analysis of XRD data. ${ }^{382}$ Although hollow fibrils were proposed as a common structure for amyloid fibrils, ${ }^{383}$ there is in now consensus that this is not the case, and $\mathrm{A} \beta$ fibrils are not hollow nanotubes, although these structures can be observed for fragments such as $A \beta(16-22)$ under appropriate conditions (section 4.5). On the other hand, MD simulations constrained by the cryo-TEM density maps for A $\beta 42$, and NMR coordinates based on data from $A \beta(17-42)$ suggest that a hollow core structure might be relevant under physiological and acidic $\mathrm{pH}$ conditions. ${ }^{384}$ 


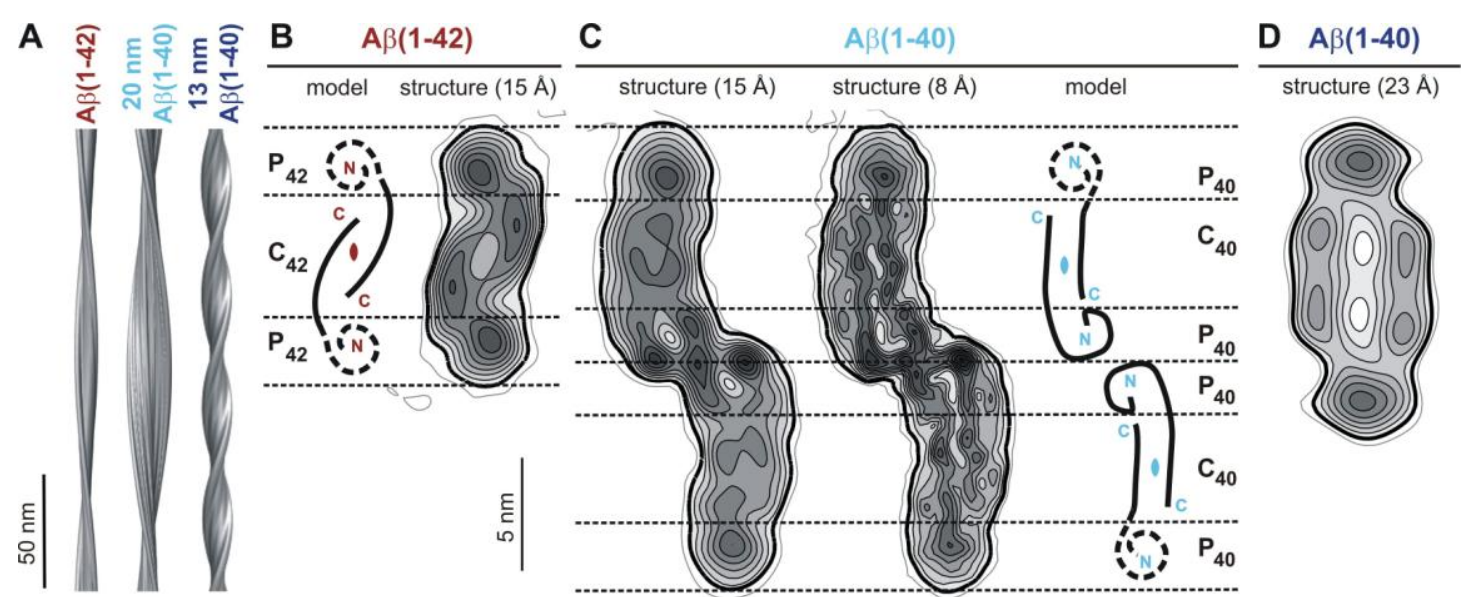

Fig.20. Electron density maps for $A \beta 40$ and $A \beta 42$, reconstructed from cryo-TEM images. ${ }^{381}$ Copyright 2009 National Academy of Sciences, USA.

The extent of unfolding of $A \beta$ and ultimately the fibril morphology seem to depend on the level of denaturation. ${ }^{385}$ By studying $A \beta 40$ and $A \beta 42$ in which the methionine (residue 35) side chain was in oxidised or reduced state, Hou et al. found on the basis of solution NMR, residue-specific interactions in the early stages of aggregation. ${ }^{386}$ These authors suggest that both hydrophobic and turn-like structures are required in the first self-assembly steps. The solution structure of the methionine-oxidised form of A $\beta 40$ has also been studied by NMR and CD by Craik and coworkers - random coil structures were observed at $\mathrm{pH} 4$ in water. ${ }^{387}$ In SDS solutions, a helical region in the $A \beta(16-24)$ is noted for this peptide. ${ }^{387}$ The specific region of $A \beta 40$ involved in contacts between fibrils has been identified by solution-state NMR, and corresponds to $\mathrm{A} \beta(15-24) .{ }^{388}$ The NMR data also show that there is an exchange between a monomeric, soluble state and an oligomeric aggregated state under appropriate (physiological) conditions of salt concentration. The equilibrium can be shifted by varying anionic strength. ${ }^{388}$ 


\subsection{Computer Simulations of $\mathrm{A} \beta$ Conformation and Aggregation}

This subject has recently been discussed in several reviews, and some aspects are mentioned in the preceeding section. ${ }^{366,389}$ Discrete molecular dynamics simulations using a four bead model (corresponding to different peptide sequences) with hydrogen bonding interactions have been used to model the oligomerization of $A \beta 40$ and $\mathrm{A} \beta 42{ }^{390}$ These simulations uncovered that the most common species are dimers for $\mathrm{A} \beta 40$ and pentamers for $A \beta 42$, although oligomers up to 9-mers were found for each. These results may be compared with the experimental data on oligomer size distribution for these two $A \beta$ peptides discussed in Section 2.6.2. Intramolecular contacts were analysed and a turn structure was proposed to stabilize the pentamer structure favoured by $A \beta 42 .{ }^{390}$ REMD has elucidated the conformation of $A \beta 42$ in which loops and turns predominate, although helical regions are found near the $\mathrm{C}$ terminus. ${ }^{391}$ It was proposed that these helices are involved in the formation of the oligomeric paranucleus revealed by ion mobility mass spectrometry (Section 2.6.2). ${ }^{391}$

The structure of $A \beta(17-42)$ protofilaments in solution has been modeled via MD simulations, along with the influence of mutations at E22 and M35. ${ }^{392}$ Steric zipper formation is observed due to favorable $\mathrm{C}$ - and $\mathrm{N}$ - terminal interface interactions. Double layered models of oligomers were constructed with association via CC or NN interfaces (Fig.21), these being stabilized by salt bridges. The U-shaped $\beta$ strand/turn/ $\beta$-strand conformation (section 4.1) leads to a hydrophobic cavity within the stacked $\beta$-sheets. ${ }^{392}$ All-atom MD simulations have been performed to elucidate the structural stability and conformational dynamics of $A \beta(9-40)$ for wild type and 
mutated sequences. ${ }^{368}$ The peptides adopt in-register parallel $\beta$-sheets. Salt bridges are formed between N23 and K28, solvated by water molecules, leading to a hydrated channel along the fibril axis. ${ }^{368}$ The results were compared to models based on solid state NMR data (section 4.1). ${ }^{360 b, 360 c}$
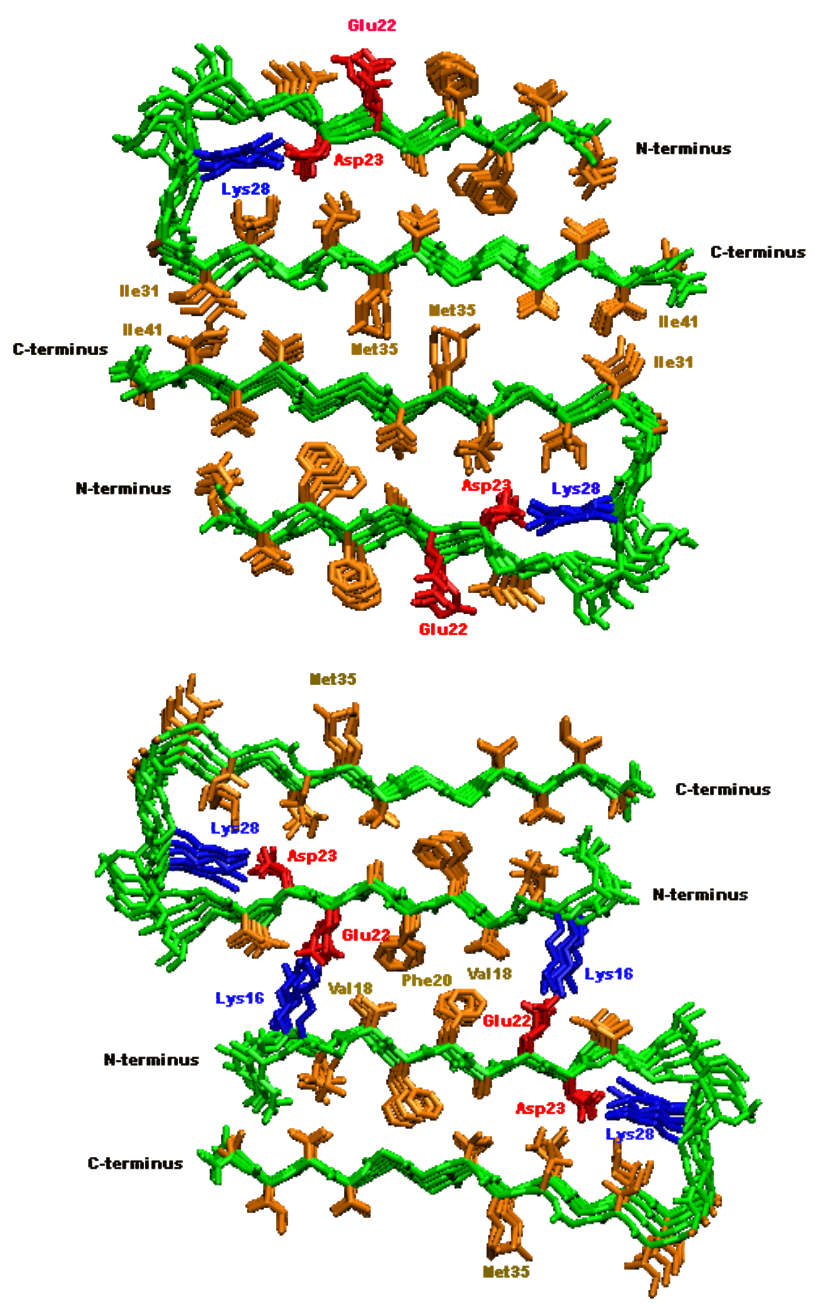

Fig.21. Double layer models used for $A \beta(17-42)$ oligomers. ${ }^{392}$ Top- association via Cterminal interfaces; Bottom- association via $\mathrm{N}$-terminal interfaces. Charged residues are shown in red and blue. Reprinted from Zheng, J. et al. Biophysical Journal 2007, 93, 3046, Copyright 2007, with permission from Elsevier. 
The oligomerization of $A \beta$ peptide fragments $A \beta(16-22)$ and $A \beta(25-35)$ has been studied by all-atom Monte Carlo simulations. ${ }^{393}$ The aggregation of the weakly hydrophobic $A \beta(25-35)$ peptide is driven by the tendency to form hydrogen bonds that stabilize $\beta$-sheets, and this peptide aggregates in a single step. The formation of a critical nucleus involving four peptides was noted for this peptide. In contrast, for the more hydrophobic $A \beta(16-22)$ peptide, initial collapse into disordered oligomers was observed. In these oligomers, hydrophobic residues are sequestered from the solvent. The subsequent reorganization of oligomers into $\beta$-sheet aggregates involves interchain H-bonding interactions and exposure of certain residues to solvent. ${ }^{393} \mathrm{MD}$ simulations have eludicated the structure of dimers formed by $\mathrm{A} \beta(25-35)$ with high $\beta$ sheet content aggregates coexisting with less structured dimers. ${ }^{394}$ The effect of the self-recognition KLVFF domain and the $\beta$-sheet breaker peptide LPFFD on the oligomerization of $A \beta(16-22)$ was examined by all atom MD simulations. ${ }^{395}$ The binding domain near the N-terminal involving H13 was identified, and binding energies were calculated. LPFFD as expected has a greater inhibitory effect on aggregation.

DMD simulations using a "united atom" model (specifying all atoms except hydrogens) have been performed to examine the conformation of $A \beta(21-30) .{ }^{367 b} \mathrm{~A}$ hairpin conformation driven by hydrophobic interactions between V24 and the butyl units of K28, and stabilized by transient salt bridging between E22/N23 and K28, was identified. ${ }^{367 \mathrm{~b}}$ REMD simulations have been carried out for the same fragment with similar conclusions. ${ }^{367 \mathrm{c}}$ The significance of the mutations at the E22 residue (Section 2.2.4) in influencing the stability of turn structures, and hence of aggregation into toxic assemblies, was noted. ${ }^{367 \mathrm{c}}$ These observations are consistent with ssNMR 
experiments as mentioned above and in the preceeding section. The results of REMD simulations on $A \beta(21-30)$ have been compared to ion mobility mass spectrometry data, in particular the collision cross-section can be modelled, providing information on conformation. ${ }^{367 \mathrm{e}}$ A particular focus was the turn structure and the influence of the E22G, E22Q, E22K and D23N mutations. ${ }^{367 e}$ The same group also performed allatom MD simulations in explicit water of the folding of $A \beta(21-30)$ and the Dutch mutant with the E22Q substitution. ${ }^{367 a}$ Similar conclusions were drawn concerning the presence of loops in the V24-K28 region in the wild-type peptide, although this is not observed for the Dutch mutant sequence. The influence of salt ions on the salt bridging interaction was examined. ${ }^{367 a}$

MD simulations have been used to investigate the adsorption of $A \beta(17-42)$ oligomers (from dimers to hexamers) onto self-assembled monolayers (SAMS) with different end-functional groups. ${ }^{396}$ The SAMS are proposed to serve as models for cell membranes. The simulations complement experimental studies (CD, AFM, SPR) on A $\beta 42$ by the same group, ${ }^{397}$ which shows that fibrillization is accelerated in the presence of SAMs, to the greatest extent for the hydrophobic $-\mathrm{CH}_{3}$ capped SAMS or the cationic $-\mathrm{NH}_{2}$ functionalized SAMS. This observation points to the role of both hydrophobic and electrostatic interactions. The simulations suggest that trimers constitute the smallest nucleus that can seed $\mathrm{A} \beta$ polymerization.

\subsection{Kinetics and Mechanisms of Fibrillization}

\subsubsection{Mechanisms}


As mentioned in Section 2.2.1, A $\beta 42$ is more aggregation prone and exhibits faster fibril growth than $A \beta 40$. A detailed model for the aggregation into initial aggregates (oligomers, termed "micelles" in the original work) and subsequently fibrils has been developed. ${ }^{398}$ This permits nucleation and elongation rate constants to be calculated.

Fibrillization of $A \beta$ appears to occur via a proto- or pre- fibrillar stage. ${ }^{6 \mathrm{~d}, 212,221 \mathrm{a}, 399}$ These oligomeric species are consumed as fibrillization proceeds. ${ }^{212,399 a}$ The prefibrillar stage has been proposed to correspond to micelle formation. Fibrillization of A $\beta 40$ occurs above a critical concentration which has been described in analogy with a critical micelle concentration (cmc) as shown in Fig.22. ${ }^{6 a, 90 b, 400}$ Fibrillization can be described using the corresponding one-dimensional model of self-assembly. ${ }^{401}$ The initially formed protofibrillar species for several proteins including $A \beta 40$ and $A \beta 42$ are spherical annular-shaped species ${ }^{212,221 \mathrm{a}, 399 \mathrm{~b}}$ which may be linked into chains. $^{221 \mathrm{a}, 399 \mathrm{~b}}$ The proto-fibril formation process may occur before or during the lag phase.

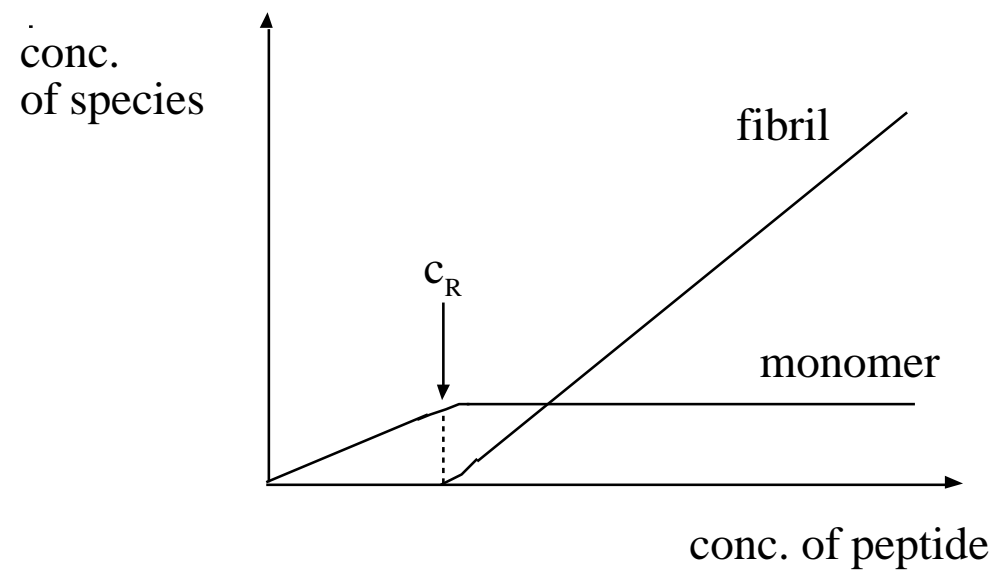

Fig.22. Amount of fibril and monomer as a function of added protein. Fibrils are formed above a critical concentration, $c_{\mathrm{R}}$. From ref. ${ }^{402}$, redrawn from ref. ${ }^{6 \mathrm{a}}$ 
Models that extend beyond the Oosawa-Asakura model ${ }^{403}$ for one-dimensional selfassembly have been developed to describe more realistically amyloid aggregation kinetics. ${ }^{404}$ In particular, the influence of fragmentation, i.e. the generation of secondary nuclei, has been considered. It will be interesting to see this model applied to further analyse the kinetics of $A \beta$ fibril growth.

It is commonly observed that fibrillization occurs after a lag phase, suggesting a nucleation and growth process. ${ }^{6 a, 90 b, 208,385 a, 400,405}$ The lag phase can be eliminated by addition of pre-formed aggregates, i.e. by seeding (Fig.23). ${ }^{6 a, 90 b}$ The influence of $\mathrm{A} \beta 40$ seeds on $\mathrm{A} \beta 42$ aggregation and vice versa has been examined using immobilized seeds. ${ }^{406}$ Oligomeric forms of the peptides were found to be more effective seeds than either monomers or fibrils and $A \beta 42$ monomers aggregated onto A $\beta 42$ fibrils more rapidly than other combinations.

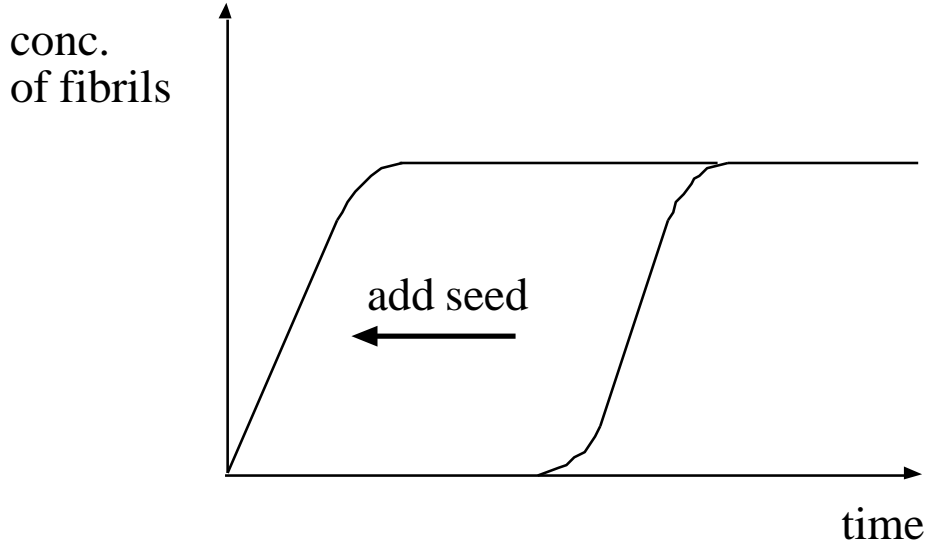

Fig.23. Addition of seed can eliminate the lag time in fibrillization. From ref. ${ }^{402}$, redrawn from ref. ${ }^{\text {6a }}$ 
$\mathrm{AFM}$ on $\mathrm{A} \beta 40$ and $\mathrm{A} \beta 42$ provide a picture of the initial formation of protofilaments followed by their replacement with fibrils, ${ }^{212}$ this technique being complemented with light scattering, and TEM and SEC analysis of the molecular weight of fractions obtained at different stages of the polymerization process. ${ }^{399 a}$

The aggregation of $A \beta$ on planar substrates, hydrophilic mica and hydrophobic graphite, has been investigated. On mica, pseudo-micellar aggregates were noted at low concentration and fibrils at higher concentration. In contrast, on graphite, sheets were observed with a thickness equal to the extended peptide length, oriented along the graphite lattice directions. ${ }^{212}$ A later AFM study examined A $\beta 42$ fibrillization and plaque formation, and the interaction between $\mathrm{A} \beta 40$ and $\mathrm{A} \beta 42 .{ }^{406}$ Deposition was studied on a synthetic template comprising an $N$-hydroxysuccinimide ester-activated solid surface. A $\beta 42$ oligomers were found to be more effective seeds for fibril growth than monomers or mature fibrils.

Lin and coworkers investigated the kinetics and mechanisms of $A \beta 40$ fibrillization under different conditions including variable temperature, ionic strength and $\mathrm{pH}^{407}$ The rate of fibrillization was enhanced with increasing temperature or ionic strength. A two-state mechanism of growth was proposed, nucleation being the ratedetermining step. The aggregation mechanism was dependent on ionic strength since addition of monomers to fibrils can occur either at the ends (at low ionic strength), or depending on screening of electrostatic interactions at high ionic strength, by lateral aggregation. ${ }^{407}$ Linse et al. suggest that $\mathrm{A} \beta 42$ (D1M mutant) fibrillization occurs via a two-phase state involving soluble $\mathrm{A} \beta$ (liquid phase) and aggregated $\mathrm{A} \beta$ (solid phase). ${ }^{408}$ They used ThT fluorescence and ELISA to monitor fibril growth, and free 
$A \beta$ concentration respectively. The free $A \beta$ concentration varied linearly with total $\mathrm{A} \beta$ concentration up to $0.2 \mu \mathrm{M}$, followed by a decrease to an asymptotic value. This suggests a $c m c$ of $0.2 \mu \mathrm{M}$ in the buffer conditions examined. ${ }^{408}$ These authors also point to the need to be extremely careful with the preparation conditions to obtain reproducible fibrillization data on $\mathrm{A} \beta 42$ due to the known complications associated with avoiding initial $A \beta$ aggregation (for example, aggregation of $A \beta 40$ even on resin during solid phase synthesis has been observed by $\operatorname{SSNMR}^{409}$ ) as well as nucleation at interfaces (air/water interface of bubbles, surface of vessel). ${ }^{408}$ It is already known that very careful protocols have to be followed in studying fibrillization of $A \beta 42$ for example, for example starting from a well-defined state of unaggregated peptide (achieved by initial dissolution in a hydrophobic solvent) and then carefully controlling the addition of water or buffer to a dried film, as discussed in Section 2.6.2. ${ }^{214}$ Agitation, e.g. via sonication, is known to have a particularly marked effect on fibril morphology and indeed can be used to create specific polymorphs. ${ }^{364,410}$

Exchange dynamics between monomers and NMR-invisible (dark state) protofibrils of $\mathrm{A} \beta 40$ and $\mathrm{A} \beta 42$ have been investigated using single residue ${ }^{15} \mathrm{~N}$ dark-state exchange saturation transfer NMR. ${ }^{411}$ This revealed that the first 8 residues at the $\mathrm{N}$ terminus exist in a mobile tethered state while the hydrophobic central residues are either tethered to, or in contact with, the protofibril surface. The $\mathrm{C}$ terminal residues display lower affinity for the protofibril surface. The ${ }^{15} \mathrm{~N}$ relaxation rates of the $\mathrm{C}$ terminus residues are larger for $\mathrm{A} \beta 42$ than $\mathrm{A} \beta 40$ and this was proposed as an explanation for the higher fibrillization rate of the former peptide. ${ }^{411}$

\subsubsection{Kinetics}


In the growth state of fibrillization, early work on $A \beta 40$ suggested that the kinetics are first order, i.e. the rate of fibril elongation is proportional to the concentration of monomers. ${ }^{400,412}$ This was confirmed via light scattering on $\mathrm{A} \beta 40$ in $0.1 \mathrm{M} \mathrm{HCl}^{400}$ (aggregation kinetics are $\mathrm{pH}$ dependent), in vitro studies of deposition onto plaques in unfixed Alzheimers disease brain tissue ${ }^{412 \mathrm{a}}$ and ThT fluorescence studies. ${ }^{412 \mathrm{~b}}$ However, in the absence of seeds, $A \beta$ aggregation exhibits faster than first order kinetics and an optimal $\mathrm{pH}$ of 5 instead of 7 as for templated aggregation. ${ }^{412 \mathrm{a}}$ The temperature dependence of fibril extension for $\mathrm{A} \beta 40$ in $0.1 \mathrm{M} \mathrm{HCl}$ (determined from size measurements via dynamic light scattering) follows the Arrhenius equation, ${ }^{413}$ with an activation energy $96 \mathrm{~kJ} \mathrm{~mol}^{-1}$, comparable to the value for unfolding of several other peptides. ${ }^{413}$ The concentration dependence of fibrillization kinetics was also analysed. Light scattering has also been used to monitor the aggregation of A $\beta 40$ in PBS at different concentrations, and the size and shape of (proto-)fibrils was analysed, ${ }^{414}$ and previously, in a similar fashion, this method was applied for $\mathrm{A} \beta(1-$ 28) ${ }^{357}$ and $\mathrm{A} \beta(1-39) .{ }^{415}$

The rate of fibrillization is strongly influenced by seeding. ${ }^{90 \mathrm{~b}}$ Fibril morphology is also influenced by seeding, as revealed by TEM and solid state NMR studies on A $\beta 40$ (see also section 4.4). ${ }^{410}$ The kinetics of $\mathrm{A} \beta$ fibril growth by covalent attachment of seeds to the surface can be monitored using using quartz crystal microbalance $(\mathrm{QCM}),{ }^{246,416}$ surface plasmon resonance (SPR $)^{417}$ or $\mathrm{AFM}^{418}$ techniques. Peptide can be immobilized on the QCM crystal via, for example, attachment of the peptide $N$ terminus to a tethered 16-mercaptohexadecanoic acid monolayer (via carbodiimide/ $N$ hydroxysuccinimide activation) or biotinylated peptide attached via avidin. ${ }^{416 \mathrm{~b}}$ The rate of elongation is observed as a change in resonant frequency of the crystal which 
correlates to an increase in surface-bound mass. In this way, monomer addition to A $\beta 40$ fibrils was studied, these were found to elongate in a reversible fashion with a rate that varies with monomer concentration and immobilized seed density. ${ }^{416 \mathrm{~b}}$ The growth was consistent with a first-order kinetic model for the single growth phase observed.

Rate constants have been determined for $A \beta$ fibril growth using several methods. Direct comparison between results from these measurements is often not possible, due to differences in the precise definition of the measured quantity. Based on 2D studies using immobilized $A \beta 40$, the following values were reported for the rates of association and dissocation: $k_{\text {ass }}=3.6 \times 10^{-4} \mu \mathrm{M}^{-1} \min ^{-1}, k_{\mathrm{dis}}=7.6 \times 10^{-5} \mathrm{~min}^{-1}$ and the association constant $K_{\mathrm{d}}=k_{\mathrm{dis}} / k_{\mathrm{ass}}=210 \mathrm{nM} .^{416 \mathrm{~b}}$ On the other hand, based on isotope exchange ESI-MS experiments on the dissociation of molecules from fibrils, the following values were determined for $\mathrm{A} \beta 42: k_{\mathrm{dis}}=0.01 \mathrm{~min}^{-1}$ for $\mathrm{A} \beta 40$ and $k_{\mathrm{dis}}=$ $1.67 \times 10^{-4} \mathrm{~s}^{-1}$ for $\mathrm{A} \beta 42 .{ }^{419}$ Linse et al. studied fibrillization of $\mathrm{A} \beta(\mathrm{M} 1-40)$ on polymeric nanoparticles and reported $k_{\text {ass }}=0.13-0.28 \mathrm{~min}^{-1}$, with a lag time $37-250$ $\min ^{114}$

Wetzel gives rate constants as shown in Table $4 .{ }^{417,420}$ These measurments were based on surface plasmon resonance measurements using immobilized seeds and may not represent bulk values.

Table 4. Rate constants for dissociation and association determined by Wetzel and coworkers $^{417,420}$ in the rapid reversible binding of monomer to the fibril in step 1 is follwed by two successive relatively slow processes (steps 2 and 3). 


\begin{tabular}{|c|l|l|}
\hline $\begin{array}{c}\text { Mechanistic } \\
\text { Step }\end{array}$ & $\begin{array}{l}k_{\text {dis }} \\
\left(\mathrm{s}^{-1}\right)\end{array}$ & $k_{\text {ass }}$ \\
\hline 1 & $8.1 \times 10^{-1}$ & $6.6 \times 10^{3} \mathrm{M}^{-1} \mathrm{~s}^{-1}$ \\
\hline 2 & $4.4 \times 10^{-3}$ & $6.4 \times 10^{-2} \mathrm{~s}^{-1}$ \\
\hline 3 & $4.3 \times 10^{-4}$ & $4.6 \times 10^{-3} \mathrm{~s}^{-1}$ \\
\hline
\end{tabular}

Lomakin et al. ${ }^{398}$ obtained for $\mathrm{A} \beta 40$ at $\mathrm{pH} 2(0.1 \mathrm{M} \mathrm{HCl})$ a fiber nucleation rate $k_{\mathrm{n}}=$ $2.4 \times 10^{-6} \mathrm{~s}^{-1}$ and elongation rate $k_{\mathrm{e}}=90 \mathrm{M}^{-1} \mathrm{~s}^{-1}$. These authors propose mechanisms of fibrillization of $A \beta 40$ under these conditions depending on whether the concentration exceeds the $c m c, c^{*}$ (Section 4.6) or not, as illustrated in Fig. $24 .^{400}$

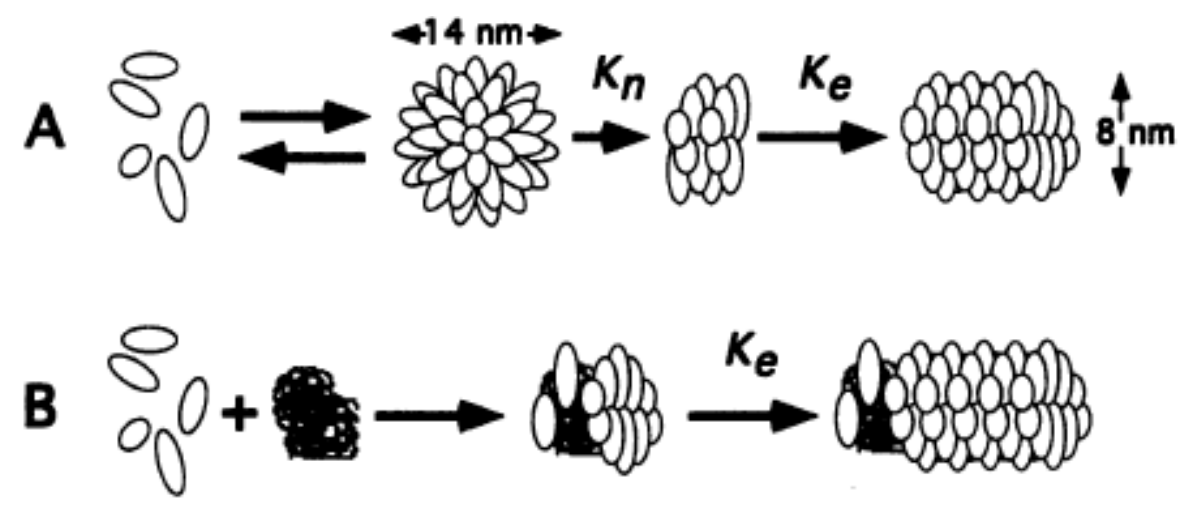

Fig.24. A) Homogeneous nucleation for $c>c m c$ via formation of initial nuclei (rate constant $k_{\mathrm{n}}$ ) followed by extension, rate constant $k_{\mathrm{e}}$. Initially monomers associate into micelles with $\left.R_{\mathrm{h}}=7 \mathrm{~nm}, \mathrm{~B}\right)$ Heterogeneous nucleation for $c<c m c$, nucleation occurs mainly on seeds not comprising A $\beta$. Figure from ref. ${ }^{400}$ Copyright 1996 National Academy of Sciences, USA. 
Inouye and Kirschner ${ }^{421}$ analysed the kinetics of Congo red binding to $\mathrm{A} \beta 40$ at $\mathrm{pH}$ 5.8 , based on the measurements by Wood et al. ${ }^{422}$ Using the Oosawa-Asakura model for one-dimensional self-assembly, they obtained values for the dissociation constant for spontaneous assembly of $K_{\mathrm{d}}=55.1 \mu \mathrm{M}$ and $k_{\mathrm{dis}}=2 \mathrm{~h}^{-1}\left(K_{\mathrm{d}}=53.7 \mu \mathrm{M}\right.$ and $k_{\mathrm{dis}}=5$ $\mathrm{h}^{-1}$ for seeded assembly) as well as the rate coefficient and proton dissociation constant. ${ }^{421}$ The authors note that the kinetics of seeded fibril formation at $\mathrm{pH} 5.8$ are faster than observed by Lomakin et al. ${ }^{400}$ at $\mathrm{pH} 2$.

\subsubsection{Thermodynamics}

Wetzel et al. have analysed the thermodynamics of amyloid fibrillization. ${ }^{420}$ The free energy of fibril elongation of wild type $A \beta 40$ was found to be approximately $-37.7 \mathrm{~kJ}$ mol $^{-1}{ }^{420}$ This group note that seeded fibril growth can be described as a dissociation/elongation equilibrium. The equilibrium is achieved with a remaining pool of monomer with a concentration of $0.7-1 \mu \mathrm{M}$ (corresponding to the $\mathrm{cmc}$ ). ${ }^{420}$ The equilibrium constant $K_{\mathrm{d}}$ was found to be around $0.8-1 \mu \mathrm{M}$ (the value being higher for mutants, and for fibril growth in the presence of ThT). A three step mechanism for elongation was proposed with three different sets of dissocation/association constants as described in the preceeding section. ${ }^{420}$ The change in Gibbs energy for a series of mutants of $A \beta 40$ was analysed in a similar fashion. ${ }^{423}$ The exchange of $A \beta$ molecules due to fibril association and dissociation has been monitored via hydrogen/deuterium exchange electrospray ionization mass spectrometry. ${ }^{419}$ Molecular recycling was found to be much more prevalent for A $\beta 40$ than for $A \beta 42$. The free energy of fibril growth for $A \beta 40$ can been determined from 
the critical concentration (Section 4.6) and this leads to $\Delta G_{e l}^{0}=-46.1 \mathrm{~kJ} \mathrm{~mol}^{-1},{ }^{424}$ in reasonable agreement with the value from Wetzel's study quoted above. The Wetzel group also studied the effect of $A \beta 40$ alanine mutations on the free energy of elongation. ${ }^{424}$ By measuring the amount of soluble monomer, and total protein concentrations at steady state for a series of amyloid forming proteins including $A \beta$ and others, the free energy of elongation was determined and these values may be compared with those for $A \beta 40 .{ }^{425}$ A correlation with the number of residues in the peptide was noted.

\subsubsection{Lack of Sequence Specificity in A BAggregation}

The cross- $\beta$ structure (Section 4.1) is a common feature for amyloids formed by many different proteins and peptides, including $A \beta .^{2 \mathrm{~d}, 39 \mathrm{~b}, 426}$ Evidence that formation of amyloid fibrils is a common state for many if not all proteins comes from several types of experiments. First, fibrils can be induced to form by partial denaturing of proteins not involved with any disease ${ }^{427}$ or using de novo designed peptide fragments (see Section 4.5). Secondly, amyloids can be induced to form by seeding with fibrils of the same, related or unrelated protein, ${ }^{6 a, 89,385 a, 405 b, 428}$ a process that may be implicated in the transmission of prion diseases, ${ }^{6 a}$ although the transmission of spongiform encephalopathies may involve cofactors in addition to prions, the full mechanism being unclear as yet. ${ }^{429}$

A study of mutants of $A \beta 40$ (including Dutch type, Section 2.2.4, but also variants for rodents compared to primates) revealed that $\mathrm{N}$-terminal substitutions that distinguish primate $A \beta 40$ from rodent $A \beta 40$ do not have a significant effect on fibril 
morphology. ${ }^{430}$ The fibrils formed by the Dutch mutant were found to have enhanced stability at high $\mathrm{pH}$ compared to the other variants.

Using fusion constructs with green fluorescent protein (GFP), libraries of mutants of wild-type $A \beta 42$ have been prepared in which hydrophobic residues at the $\mathrm{C}$ terminus ${ }^{431}$ or $8-12$ other residues in the $\mathrm{C}$ terminal domain ${ }^{432}$ have been substituted with random nonpolar residues. The folding and fluorescence of GFP is prevented by $\mathrm{A} \beta 42$, and mutations in $\mathrm{A} \beta 42$ that disrupt aggregation then lead to increased fluorescence. ${ }^{433}$ It was shown that fibrillization is promoted with hydrophobic residues at positions 41 and $42 .{ }^{431}$ and all the hydrophobic mutations (8-12 residues). ${ }^{432}$ This implies that generic hydrophobic sequences may be sufficient to promote $A \beta 42$ fibrillization. ${ }^{432}$ In related work, A $\beta 42-G F P$ fusion constructs with A $\beta 42$ mutations across the whole sequence were expressed and $A \beta 42$ aggregation was probed. This also identified key hydrophobic sequences involved in fibrillization. ${ }^{433}$

\subsection{Polymorphism}

The morphology of $A \beta$ fibrils can be controlled through the growth conditions, and seeding, leading to distinct polymorphism. This in turn is related to the selection of specific conformations. ${ }^{389 \mathrm{~b}}$ An important contribution on the subject of polymorphism was the study by Petkova et al. ${ }^{410}$ on A $\beta 40$ polymorphism, controlled through application of sonication or not, and imaged by TEM (Fig.25). Solid state NMR was used to identify cross-correlations between different residues, i.e. to probe 
conformations in different polymorphs. The cytotoxicity towards rat neurons was also shown to depend on A $\beta 40$ polymorphism. In vivo, the phenotype of seeded amyloidosis depends on both the source and the host. ${ }^{89}$ This was examined using APP23 and APPPS1 transgenic mice, which overexpress A $\beta 40$ and A $\beta 42$ respectively. It was suggested that the dependence of amyloid morphology on the seed indicates the existence of $A \beta$ polymorphism with associated distinct polymorph biological activity, reminiscent of prion strains. ${ }^{89}$

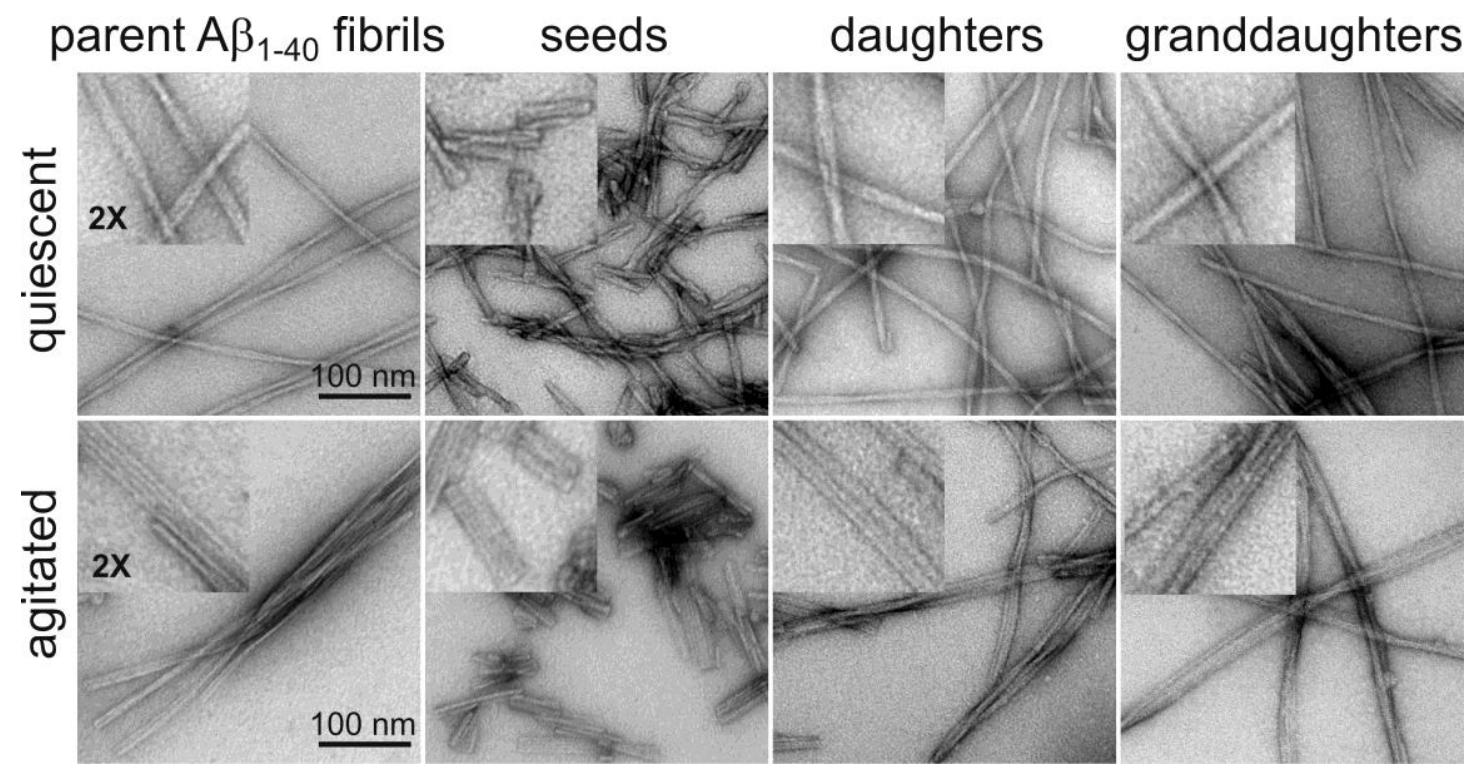

Fig.25. Polymorphism of A $\beta 40$ fibrils. ${ }^{410}$ From Petkova, A. T. et al., Science 2005, 307, 262. Reprinted with permission from AAAS.

Tycko's group have recently shown that the polymorphism of $A \beta 40$ (Iowa mutant) can be reduced by repeated seeding (up to nine generations were studied), which can be used to amplify a particular polymorph, even when it is initially a minor component. $^{364}$ 
Seeds comprising nanotubes (self-assembled in methanol) of the modified peptide AAKLVFF (containing A $\beta(16-20)$, KLVFF) can seed nanotube formation in aqueous solution (conditions that favour non-nanotube fibrillization of unseeded peptide). ${ }^{434}$ TEM was complemented by solid-state NMR which probed differences in the strand registry and residue packing in the nanotubes and fibrils. These observations point to the non-equilibrium nature of the seeded polymorphism.

The factors that can influence amyloid fibril polymorphism are complex, and apart from factors such as agitation during sample preparation, the presence of hydrophobic interfaces or the air-water interface can influence fibrillization. ${ }^{435}$

\subsection{Fragments}

The fibrillization of fragments of $A \beta 40$ and $A \beta 42$ has been extensively investigated. Several early studies are summarized by Teplow ${ }^{6 \mathrm{~b}}$ and Serpell. ${ }^{27 \mathrm{~d}}$

Aggregation-prone sequences in $A \beta$ have been identified by computer modelling of measures of $\beta$-sheet forming propensity. The TANGO algorithm, based on a statistical mechanical model of protein conformation (based on measures of propensity to form defined secondary structures), predicts a strong tendency for aggregation of $A \beta(17-21)$ and $A \beta(31-36)$, with enhanced aggregation of $A \beta 42$ compared to $\mathrm{A} \beta 40 .{ }^{436}$ It can also account for the aggregation propensity of some of the mutant forms of $A \beta$ (Section 2.2.4). The Zyggregator algorithm is based on measures such as hydrophobicity and patterning of hydrophobic residues that lead to a parameter set obtained by screening of sequences in protein databases. ${ }^{437}$ It predicts a strong tendency for aggregation of $A \beta(15-23)$ and $A \beta(30-42)$, i.e. similar domains as 
indicated by TANGO. ${ }^{202 c, 437}$ These predictions have been compared to results from solid state NMR experiments for both protofibrils and mature fibrils and protofibrils are found generally to have shorter aggregation domains. ${ }^{438}$

Several key regions are associated with aggregation propensity, including the transmembrane hydrophobic domain and the C-terminal hydrophobic domain. In the following, we focus on fibrillization by the shortest core fragment critical to fibril formation which is believed to lie in the $A \beta(16-22)$, KLVFFAE, region. Sequences in this central region of $A \beta 42$ are of great interest because cleavage by the enzyme $\alpha$ secretase occurs between $\mathrm{K}$ and $\mathrm{L}^{2 \mathrm{a}}$ Hilbich et al. showed that a region in the hydrophobic core around residues 17 to 20 , i.e. LVFF, is crucial for $\beta$-sheet formation. ${ }^{439}$ They prepared variants of $A \beta 43$, with substitution of various residues 17 to 20, and investigated fibrillization by CD, FTIR and TEM. Substitution with hydrophilic amino acids led to a significant reduction in amyloid formation. Tjernberg et al. studied the binding of fragments and variant fragments of $A \beta 40$ to the full peptide. ${ }^{440}$ Binding of ${ }^{125}$ I-labelled $A \beta 40$ was studied by autoradiography. A series of fragments of $A \beta 40$ ranging from 3 to 10 residues was prepared. Only pentapeptides or longer showed significant binding to $A \beta 40$, and fragment $A \beta(16-20)$, i.e. KLVFF, is contained in all strongly binding sequences. ${ }^{440 \mathrm{~b}}$ By preparing pentapeptide variants of KLVFF with substituted amino acids, it was found that residues 1, 2 and 5 (K, L, F) are most important for binding of this fragment to $A \beta 40 .{ }^{440} \mathrm{~A}$ model for the binding of KLVFF to $A \beta(13-23)$ confirmed the importance of these residues in forming an anti-parallel $\beta$-sheet. The binding capacity of pentapeptides containing D-amino acids instead of L-amino acids was also studied, since the latter are resistant to proteolysis. Residues 2 and 3 were found to be most critical for binding, D-Lys, D-Phe enhancing 
binding. ${ }^{440 \mathrm{~b}}$ Findeis et al. presented a very thorough study on $\mathrm{A} \beta$-based inhibitors of $A \beta$ fibrillization. ${ }^{280}$ This revealed again the importance of the $A \beta(16-21)$ region. $A$ derivative of $\mathrm{A} \beta(17-21)$, cholyl-LVFFA-OH was found to be a particularly potent inhibitor of fibrillization, although with limited biochemical stability. The D-amino acid version however was found to be stable in monkey cerebrospinal fluid. Other researchers have explored the role of molecular architecture on the inhibition of fibrillization. Dendritic tetramers containing terminal KLVFF motifs inhibit the aggregation of low molecular weight and protofibrillar $A \beta(1-42)$ into fibrils, substantially more than the linear KLVFF peptide. ${ }^{441}$ Recently, bifunctional molecules with terminal KLVFF pentapeptides linked by aminohexanoic acid spacers, have been shown to recognise early $A \beta$ oligomers, manly trimers and tetramers, in mixtures. ${ }^{442}$ The molecules may act as "tweezers", binding small oligomers if the central spacer has the correct length.

The dependence of fibrillization on fragment size was investigated for $A \beta$ fragments containing the $A \beta(16-20)$ sequence. ${ }^{443}$ Electron microscopy suggested that the shortest fibril-forming sequence was $A \beta(14-23)$, i.e. the decapeptide HQKLVFFAED. The KLVFF sequence was found not to form fibrils itself. However, more recent work shows that this peptide does form $\beta$-sheet fibrils under appropriate conditions in aqueous solution. ${ }^{444}$ Meredith and coworkers later studied variants of $\mathrm{KLVFF}^{284}$ and $\mathrm{KLVFFAE}^{445}$ in which amide protons in alternate residues were replaced by $N$-methyl groups. ${ }^{284,445} \mathrm{Ac}-\mathrm{K}(\mathrm{Me}) \mathrm{LV}(\mathrm{Me}) \mathrm{FF}-\mathrm{NH}_{2}$ was shown to form extended $\beta$-strands. ${ }^{284}$ It is also more water soluble than KLVFF and can permeate phospholipid vesicles and cell membranes. This variant is also resistant to denaturation by addition of solvent, increase of temperature or $\mathrm{pH}$. It is also a potent inhibitor of $\mathrm{A} \beta 40$ fibrillization, and 
can break up preformed $A \beta 40$ fibrils, being more effective than KLVFF in both $\operatorname{regards}^{284}$ (as is heptapeptide $\mathrm{NH}_{2}-\mathrm{KLV}(\mathrm{Me}) \mathrm{F}(\mathrm{Me}) \mathrm{F}(\mathrm{Me}) \mathrm{A}(\mathrm{Me}) \mathrm{E}-\mathrm{CONH}_{2}{ }^{445}$ ). These fragments are believed to form $\beta$-strands with distinct faces - one with unmodified groups capable of forming hydrogen bonds and the other containing non-polar methyl groups. This can disrupt the hydrogen bonded $\beta$-sheet structure of the $\mathrm{A} \beta$ peptide itself.

Other fragments have been designed to inhibit $\mathrm{A} \beta 40$ and $\mathrm{A} \beta 42$ fibrillization. Rational design principles based on the knowledge of the pentapeptide binding sequence led to a study on LPFFD, ${ }^{446}$ now known as the $\beta$-sheet breaker peptide. This peptide incorporates proline, known to be a $\beta$-sheet blocker and was found to reduce amyloid deposition in vivo (rat model) and to disassemble pre-formed fibrils in vitro. ${ }^{446}$ Prior investigations of $A \beta(12-26)$ with proline substitutions had shown that Pro replacement of any residue in the $A \beta(17-23)$ LVFFAED sequence leads to a loss of fibril formation ${ }^{447}$ and this was confirmed via F19P substitution in $A \beta 42$, although oligomeric species were still detected. ${ }^{217}$ This observation also points to the role of Pro residues in forming the core of $\beta$-sheets. The capped version of the $\beta$-breaker peptide, $\mathrm{CH}_{3} \mathrm{CO}-\mathrm{LPFFD}-\mathrm{CONH}_{2}$, has been shown to improve spatial learning in a rat model. $^{448}$

The retro-inverse peptide ffvlk (lower case indicates D-amino acids) binds A $\beta 40$ fibrils with moderate affinity but this binding can be significantly enhanced by attaching multiple copies of this peptide to an eight-arm branched PEG. ${ }^{449}$ Tandem dimers of ffvlk linked by a $\mathrm{k}(\beta \mathrm{A})$ (k denotes $\mathrm{D}$-lysine) spacer or a difunctional PEG 
chain also showed some enhancement of binding. All of these conjugates are effective in inhibiting fibrillization of the full $\mathrm{A} \beta 40$ peptide. ${ }^{449}$

TEM indicated that KLVFF itself forms fibrils in aqueous PBS solutions ( $\mathrm{pH} 7.4),{ }^{284}$ contrary to the reports by Tjernberg et al. ${ }^{443}$ There existed some controversy as to whether this fragment itself fibrillizes. This was resolved in a study which used cryogenic-TEM (cryo-TEM) among other techniques to confirm that KLVFF does form amyloid fibrils in aqueous solution, at sufficiently high concentration. ${ }^{444}$ CryoTEM importantly avoids artifacts when drying to prepare samples for conventional negative stain TEM, this can be problematic in the case of weakly fibrillizing peptides. Fibril formation has been reported for the heptapeptide $\mathrm{A} \beta(16-22), \mathrm{CH}_{3} \mathrm{CO}$ KLVFFAE-NH ${ }_{2}{ }^{375}$ It has been suggested that "fibrils" of this peptide actually comprise nanotubes, based on electron microscopy, atomic force microscopy and small-angle scattering data, and a detailed model for the lamination of the $\beta$-sheets in the nanotube wall has been proposed. ${ }^{450}$ The related capped peptide $A \beta(16-22) \mathrm{E} 22 \mathrm{~L}$ self-assembles into nanotubes in water/acetonitrile solution and the grooved surfaces of these were used to probe the binding of Congo red, which was found to bind parallel to the long axis. ${ }^{451}$ Analysis of the 3D structure of $A \beta 42$ from NMR (discussed in detail in section 4.1) indicates that residues $A \beta(18-26)$ form a $\beta$-sheet as do residues 31-42 within the overall $\beta$-strand/turn/ $\beta$-strand structure of residues $18-42$ as shown in Fig.19 (residues 1-17 are disordered). ${ }^{365}$ The sequence $A \beta(17-23)$ which seems to be vital in amyloid self-assembly has also been shown to be important in forming the correct $\beta$-pleated sheet structure of the $A \beta$ peptide. ${ }^{439,447}$ As discussed in section 4.2, computer modelling predicts that $A \beta(17-21)$ should be prone to $\beta$-sheet aggregation..$^{202 c, 452}$ Using algorithms based on the aggregation properties of the 
constituent amino acids, Kallberg et al. ${ }^{453}$ suggest that $\mathrm{A} \beta(16-23)$ is a so-called discordant sequence of amino acids, in the sense that this sequence is predicted to adopt a $\beta$-strand conformation, whereas the full protein structure in the protein data base (ref 1ba6) indicates an $\alpha$-helix for this region of $A \beta 40$. The protein database structure $1 \mathrm{ba} 6^{387}$ is for A $\beta 40$ with oxidised methionine (residue 35 ) in aqueous SDS solution, a solvent which is known to favour $\alpha$-helices, observed between residues 16 and 24 (section 4.1). NMR data on A $\beta 40$ in aqueous solution also indicate an $\alpha$-helix for residues 15-24 in a water/TFE solution ${ }^{454}$ and for residues 1-36 in SDS solution ${ }^{455}$ (the data from Sticht et al. ${ }^{454}$ gives pdb structure 1AML). As mentioned in Section 4.1, NMR in aqueous solution ${ }^{365}$ indicates a $\beta$-sheet in this region of $A \beta 42$. Different methods to predict secondary structure indeed lead to different predictions for the conformation of KLVFF. The method of Garnier predicts $\alpha$-helices for KLVFF, whereas the Chou-Fasman method predicts residues KLV are in $\beta$-strand and FF in $\alpha$ helix structures. ${ }^{202 \mathrm{c}} \mathrm{A} \beta(9-11)$, i.e. $\mathrm{NH}_{2}-\mathrm{GYE}-\mathrm{OH}$, forms amyloid-like fibrils in aqueous solution. ${ }^{456}$ MTT assays indicate that the peptide is toxic to neurons.

$\mathrm{N}$-terminal variants of $\mathrm{A} \beta$ may also play an important role in $\mathrm{AD}$ pathophysiology. ${ }^{192,457}$ Hilbich et al. used CD and EM to investigate the fibril-forming properties of $A \beta 43$ and $N$-terminal truncated variants along with variants of $A \beta(10-23)$ with substitution of hydrophobic residues. ${ }^{361}$ Peptides in which phenylalanine residues are substituted for less hydrophobic residues show an enhanced solubility in salt solution compared to the native sequence. These authors also investigated variants in which pairs of residues (D23K28, V24G29, G25A30) in the $A \beta(10-43)$ peptide were replaced by pairs of cysteines, in order to examine the influence of disulfide bridging, 
i.e. to fix an artificial turn structure. As discussed in section 4.1, the native peptide is believed to adopt a turn structure in the $\mathrm{A} \beta(23-30)$ domain. ${ }^{361}$

The fibrillization of $\mathrm{A} \beta$ variants with $\mathrm{N}$-terminal glutamines replaced with pyroglutamyl residues has been investigated. Peptides with this form of posttranscriptional modification are observed in vivo, in the brains of AD and Down's syndrome patients ${ }^{457-458}$ due to presenilin 1 mutations. ${ }^{55 a}$ In particular, the aggregation of pGlu-A $\beta(3-42)^{458}$ and pGlu-A $\beta(11-42)$ has been examined. ${ }^{459}$ These peptides exhibit accelerated aggregation compared to the unmodified $A \beta 40$ and $A \beta 42$ and their possible role in seeding aggregation in vivo was noted. ${ }^{459}$

The important role of the C-terminal hydrophobic domain, in particular $A \beta(34-42)$ in driving fibril formation has been examined. ${ }^{34,372,460}$ The length of the $\mathrm{C}$ terminus critically influences the rate of amyloid formation but only has a minor effect on the solubility. ${ }^{34}$ Peptides containing the $C$ terminal sequence $A \beta(36-42 / 43)$ can seed fibrillization by peptides lacking the $\mathrm{C}$ terminal residues $(\mathrm{A} \beta 40-42){ }^{34}$

\subsection{Micelles}

$\mathrm{A} \beta$ has surfactant-like properties, for example it is able to reduce surface tension in a concentration-dependent manner and both $A \beta 40$ and $A \beta 42$ and shorter variants $(C$ terminal truncations) exhibit an apparent critical micelle concentration $(\mathrm{cmc}){ }^{461}$ The "micelles" may in fact correspond to proto-fibrillar species believed to be involved in the nucleation of fibril formation (section 4.3.1). The formation of SDS-stable aggregates was also confirmed by SDS-PAGE. For a series of C-truncated peptides, the $c m c$ was found to be $25 \mu \mathrm{M}$ in aqueous solution, and measurements on the 
partitioning of a fluorescent dye DPH (1,6-diphenyl 1,3,5-hextriene) suggested that residues 29-42 form the interior hydrophobic domain. ${ }^{461}$ The $c m c$ of $A \beta 40$ has also been deduced from the concentration dependence of fibril growth kinetics and was found to be $c^{*}=0.1 \mathrm{mM}$ in acidic aqueous solution. ${ }^{400}$ Surface-pressure area experiments and pyrene fluorescence measurements indicate $c^{*}=17.5-17.6 \mu \mathrm{M}$ in aqueous Tris buffer solution. ${ }^{462}$ Wetzel and coworkers report a value $c^{*}=0.7-1 \mu \mathrm{M}$ in aqueous PBS solution, measured by SPR and also deduced from fibril dissociation equilibrium constants, obtained from kinetic measurements of ThT fluorescence. ${ }^{420}$ Linse et al. obtained $c^{*}=0.2 \mu \mathrm{M}$ for $\mathrm{A} \beta(\mathrm{M} 1-42)$ in the buffer system they studied, and noted that this is lower than the value observed by Wetzel and coworkers for A $\beta 40$ due to differences in ionic strength of buffer as well as the peptide length. ${ }^{408}$ The aggregation number of the micelles $(\mathrm{N}=25)$ was estimated using fluorescence quenching techniques. The $c m c$ of $A \beta(11-25)$ determined from FRET experiments is $3 \mu \mathrm{M}$ at $\mathrm{pH} 5$ and $70 \mu \mathrm{M}$ at $\mathrm{pH} 7.4 .^{463}$

Above the cmc, Lomakin et al. found that the initial rate of elongation and the final size of fibrils were independent of $A \beta$ concentration due to the monomer-micelle equilibrium. ${ }^{400}$ The surfactant $n$-dodecylhexaoxyethylene glycol monoether $\left(\mathrm{C}_{12} \mathrm{E}_{6}\right)$ slowed nucleation and elongation of $A \beta 40$ fibrils in a concentration-dependent manner. ${ }^{400}$ The hydrodynamic radius of the micelles was approximately $7 \mathrm{~nm}$. Detailed SANS experiments later provided a model for the shape and dimensions of "spherocylindrical" micelles. ${ }^{464}$ The authors identified these species as aggregates comprising 30-50 monomers, therefore they seem to correspond to proto-fibrils rather than oligomers. 
SDS micelles hinder the formation of $\beta$-sheet fibrils by $A \beta 40$ and $A \beta 42$, and instead stabilize helical conformations. ${ }^{373}$ However, at lower concentration (below the $\mathrm{cmc}$ ), SDS promotes the formation of oligomers by A $\beta 42$ (not A $\beta 40)$. ${ }^{465}$ The interaction of A $\beta 40$ with SDS has been investigated by small-angle X-ray and neutron scattering (SAXS and SANS). ${ }^{466}$ It was shown by time-resolved SAXS that A $\beta 40$ rapidly forms a complex with pre-existing SDS micelles due to interaction between the sulfate groups and the hydrophilic headgroup units of the surfactant. ${ }^{466}$ Below the critical micelle concentration of SDS, globular core-shell aggregates were also formed but lagging behind the $C D$-observed transition in secondary structure of $A \beta 40$ from random coil to (predominantly) $\alpha$-helical. These measurements also showed that aggregation of $\mathrm{A} \beta 40$ in the presence of $\mathrm{HCl}$ can be monitored by SAXS. Aggregates of A $\beta 42$ with SDS (sub-micellar concentrations) have been observed via AFM, and the effect of SDS on secondary structure was probed by CD and FTIR. ${ }^{467}$ The cationic surfactant hexadecyl- $N$-methylpiperidinium bromide is able to inhibit $\mathrm{A} \beta$ aggregation well below its $\mathrm{cmc}$, pointing to a mechanism not involving micellar solubilisation. ${ }^{468}$ A similar phenomenon is reported for a tetrameric quaternary ammonium cationic surfactant which can also disassemble existing A $\beta 40$ fibrils. ${ }^{469}$ Instead, it was suggested that a specific binding surface on $A \beta 40$ (absent for other amyloid forming proteins) is able to bind such amphiphilic molecules. ${ }^{468}$ A cationic surfactant containing an azobenzene moiety influences $A \beta 40$ fibrillization differently depending on its conformation, which can be photo-switched. ${ }^{470}$ The interaction of $A \beta(12-28)$ with SDS has been investigated by CD and NMR, an $\alpha$-helical conformation for residues 16-24 was noted (see also section 4.1). ${ }^{374}$ 
The interaction of $A \beta 40$ with the bio-derived surfactant surfactin is also dependent on the aggregation state of the surfactant. ${ }^{471}$ Below the $c m c$, surfactin causes $A \beta 40$ to unfold and to fibrillize. Well above the $c m c$, $\beta$-sheet fibril formation is inhibited.

\subsection{Interactions with Lipid Membranes}

The importance of lipid interactions with $A \beta$ is highlighted by the fact that ApoE, (especially the $\varepsilon 4$ allele) a key genetic risk factor for $\mathrm{AD}$ (section 2.1), is involved in lipid metabolism. ${ }^{472}$ Lipid membranes have a number of important roles in modulating amyloid fibrillization. These include: (partially) unfolding the peptide, increasing the local concentration of peptide bound to the membrane, orienting the bound protein in an aggregation-prone manner and variation of penetration depth into the membrane affecting the nucleation propensity. ${ }^{473}$ Lipid rafts are implicated in $\mathrm{A} \beta$ dimer and oligomer formation. ${ }^{474}$ and may provide platforms for selective deposition of different $A \beta$ aggregates (this also depends on the ordering of the lipids within the membranes which may be different in the rafts $\left.{ }^{475}\right) .{ }^{476}$ Ganglioside-rich lipid rafts induce $A \beta$ oligomerization, for which cholesterol appears not to be essential. ${ }^{474 b}$ The involvement of different types of membrane structure (vesicles formed by different phospholipids and gangliosides) in $A \beta$ fibril growth has been discussed in reviews of membrane-amyloid interactions. ${ }^{224 \mathrm{~g}, 473,477}$ The role of membranes in the formation of annular structures that may comprise arrays of oligomers has been revealed by AFM. ${ }^{224 g, 226}$ and the role of oligomers in creating pores/ion channels has been

revealed by membrane conductance measurements (see also section 2.7). ${ }^{215 a}$ 
$A \beta$ is generated via regulated intramembrane proteolysis (RIP), ${ }^{6 e}$ which involves the shedding of the ectodomain of APP through membrane-anchored secretases (section 2.2.3). The membrane-bound stubs can then be cleaved within their transmembrane domains to release small peptides (A $\beta$ in the case of APP) into the extracellular space and intracellular domains into the cytoplasm. ${ }^{6}$

$\mathrm{A} \beta$, which is a cationic peptide at neutral $\mathrm{pH}$, (residue-specific $\mathrm{pKa}$ values are available ${ }^{374,478}$ ) interacts with anionic lipid membranes through electrostatic interactions, depending on $\mathrm{pH}^{224 \mathrm{~g}, 477 \mathrm{a}}$ On the other hand, it has been reported that $\mathrm{A} \beta$ can interact with cationic or zwitterionic lipids as readily as anionic lipids. ${ }^{479}$ This suggests that association of $A \beta$ with lipid membranes is driven to a substantial extent by hydrophobic interactions with hydrophobic regions in the peptide. Preferential incorporation of $A \beta$ into anionic lipid membranes is however noted. ${ }^{480}$ The interaction of $\mathrm{A} \beta$ with negatively charged lipids is driven by electrostatic interactions whereas insertion into the membrane is driven by the hydrophobic tail of $A \beta$. It has been demonstrated that $A \beta 40$ spontaneously inserts into anionic DPPG (1,2-dipalmitoyl phosphatidylglycerol) membranes but not zwitterionic DPPC (1,2-dipalmitoyl phosphatidylcholine) membranes. ${ }^{481}$ Furthermore, the DPPG membrane induces $\beta$ sheet "crystallization" of $\mathrm{A} \beta,{ }^{481}$ although this interaction was eliminated above $\mathrm{pH} 7.4$ where $A \beta$ becomes anionic. Enhanced binding of $A \beta 40$ (tryptophan labeled Y10W for fluorescence experiments) oligomers to vesicles has been observed when DPPG is incorporated as compared to pure DPPC vesicles. ${ }^{482}$ Terzi et al. studied A $\beta(25-35)$ [and $\mathrm{A} \beta(25-35 \mathrm{Nle})]$ on anionic lipid membranes and noted an increase in $\beta$-sheet formation (in solution both random coil and $\beta$-sheet structures coexist for this peptide) 
as probed by CD spectroscopy, in the presence of the negatively charged vesicles. ${ }^{483}$ These authors were also able to measure binding enthalpies. ${ }^{483}$ The $\mathrm{C}$ terminus capped version of this peptide however adopts a random coil structure on binding to the lipid vesicles, pointing to the role of electrostatics in the binding process, also confirmed by salt screening experiments. ${ }^{483}$ This group found that $A \beta 40$ can insert into anionic monolayers at sufficiently low packing density. ${ }^{480}$ These authors also noted transitions in secondary structure random coil $-\beta$-sheet $-\alpha$-helix depending on the lipid-topeptide ratio. ${ }^{480}$ A shift to an initial $\alpha$-helical conformation has been noted upon binding of $A \beta$ peptides to membranes, ${ }^{374}$ however the enrichment of peptide concentration close to the membrane may subsequently favour $\beta$-sheet formation. ${ }^{224 \mathrm{~g}, 477 \mathrm{a}}$ Thus, membranes can inhibit fibrillization at high lipid-to-peptide ratio, but accelerate it at low relative lipid concentration. ${ }^{224 \mathrm{~g}}$ Biological lipids (in liposomes) can resolubilize $\mathrm{A} \beta 42$ fibrils, and convert them into more toxic oligomeric forms, and the potential biological relevance of this was noted. ${ }^{484}$

$\mathrm{A} \beta$ can form cation-selective channels when incorporated in lipid bilayers as revealed by conductance measurements. ${ }^{225 a-c, 485} \mathrm{~A} \beta$ peptides disrupt membranes comprised of negatively charged phospholipids, in a pH-dependent manner (which is important in the context of different $\mathrm{pH}$ levels in endosomes vs. the extracellular matrix, for example). ${ }^{486}$ AFM shows the formation of channel structures by $A \beta 42$ reconstituted in planar lipid bilayers. ${ }^{226 a}$ On the other hand, Kayed et al. report that A $\beta$ oligomers increase permeability (quantified via conductance measurements) without any evidence for pore formation or ion selectivity. ${ }^{215 a}$ Serpell and coworkers have observed that the $A \beta$ aggregation state influences its ability to permeate vesicles (probed via calcein fluorescence measurements), and that oligomers have the highest 
membrane disruption activity. ${ }^{487}$ The less fibrillogenic $A \beta 40$ has a reduced tendency to permeabilize membranes than A $\beta 42$. The role of GM1 ganglioside receptors was also highlighted. ${ }^{487}$

Nussinov and coworkers have performed MD simulations of A $\beta$ in lipid bilayers, focussing on $A \beta(17-42)$ protofibrils, and $A \beta$ pore structures were examined. ${ }^{366,488}$ The formation of subunit structures within the channels was observed. The selectivity of the channels for $\mathrm{Ca}^{2+}$ observed experimentally was also confirmed from the models. ${ }^{488}$ Consistent with AFM images of A $\beta 40$ in a DOPC (dioleoyl phosphatidylcholine) bilayer, ${ }^{226 \mathrm{~b}}$ break-up of the channels into subunits was observed (Fig.26). ${ }^{488}$ Strodel et al. have also performed MD simulations on $\mathrm{A} \beta$ pore structures, for $\mathrm{A} \beta 42$, modeling oligomers. ${ }^{489}$ They found that membrane-spanning $\beta$-sheets adopt ordered configurations for dimers to hexamers, however separation into subunits was only observed for octameric oligomers which separated into distinct subunits ( $c f$. Fig.24). ${ }^{489}$ A molecular dynamics simulation of the interaction between $A \beta 42$ and zwitterionic and anionic lipids indicated that peptide-peptide interactions are favoured in the vicinity of the membrane, driving oligomerization in the case of the anionic lipid membranes. ${ }^{490}$ The dependence on $\mathrm{pH}$ was also modelled. 


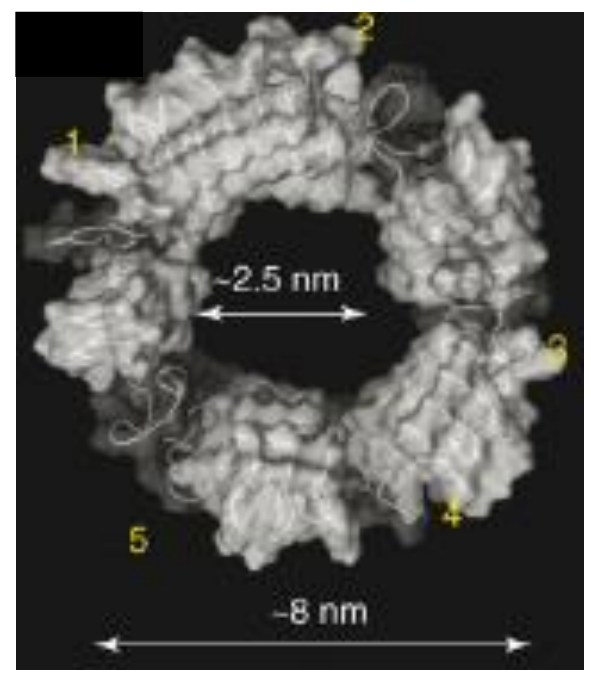

Fig.26. MD simulation of a 24-mer channel formed by $A \beta(17-42)$ in a DOPC lipid bilayer. ${ }^{366,488}$ The yellow numbers label sub-units. Reprinted from Jang, H. B. et al. Trends in Biochemical Science 2008, 33, 91, Copyright 2008, with permission from Elsevier.

The binding of $A \beta$ and gangliosides [ganglioside = glycosphingolipid] has been the subject of several studies, although with conflicting conclusions. ${ }^{477 a, 491}$ Ganglioside membranes have been reported to accelerate $A \beta$ aggregation ${ }^{300,474 c}$ or to inhibit it and stabilize $\alpha$-helical structures ${ }^{492}$ or to induce $\beta$-sheet structure. ${ }^{493}$ Another report claims that gangliosides induce $\mathrm{A} \beta$ to adopt a mixed $\alpha / \beta$ conformation at neutral $\mathrm{pH}^{486}$ Actually, a transition from random coil to $\alpha$-helix might be favoured at low peptideganglioside lipid ratios, whereas higher ratios promote the adoption of a $\beta$-sheet conformation. ${ }^{24 \mathrm{~g}, 493 \mathrm{~b}}$ It has been proposed that ganglioside clusters may form sites that seed $A \beta$ fibril formation. ${ }^{224 \mathrm{~g}, 477 \mathrm{a}}$ The interaction of $A \beta 40$ and $A \beta 42$ with phosphatidylinositol (PI) with different inositol headgroups was examined. ${ }^{276}$ The 
formation of $\beta$-sheet structures was found to be $\mathrm{pH}$-dependent in the presence of PI vesicles - both $\mathrm{A} \beta 40$ and $\mathrm{A} \beta 42$ aggregate at $\mathrm{pH} 6$ (close to the $\mathrm{pI}=5.5)^{491,494}$ but only A $\beta 42$ aggregates at $\mathrm{pH} 7$. The work with different PI vesicles formed part of the basis for the development of an inositol-based inhibitor of $A \beta$ aggregation, ${ }^{276}$ as discussed further in Section 3.2.1. formation of $A \beta 40$ fibrils is accelerated in the presence of ganglioside-containing POPC (1-palmitoyl-2-oleoyl phosphatidylcholine) vesicles. ${ }^{300,495}$ The binding of the peptide to the membrane was analysed quantitatively. The binding was found to be ganglioside-specific. ${ }^{300,495}$ AFM and TEM imaging show aggregation and fibril formation of $A \beta 40$ and $A \beta 42$ on total brain extract lipids. $^{496}$

Bokvist et al. used MAS-NMR (MAS: magic angle spinning) and CD to probe the interaction of $\mathrm{A} \beta 40$ in different lipid membranes. ${ }^{497}$ Charged membranes can act as templates for aggregation of surface-associated $A \beta 40$ in the case that the peptide is released in soluble form. However, membrane inserted A $\beta 40$ is anchored by the K28 residue by electrostatic interactions with negatively charged lipids. ${ }^{497-498}$ By studying a series of full length peptides and fragments, Chauhan et al. were able to highlight the role of aliphatic residues at the $C$ terminus of $A \beta$ interacting with the fatty acid chains as well as the electrostatic interaction involving K28. ${ }^{498}$ Several groups have noted that the interaction of $A \beta$ with membranes depends on the ordering of the lipid membranes. ${ }^{479 b, 499}$ Murphy and coworkers used fluorescence anisotropy of an inserted probe to examine interaction of $A \beta 40$ with lipid membranes. ${ }^{479 b, 491}$ They observe that A $\beta$ aggregates only affect lipid membrane fluidity above, and not below, the lipid chain melting temperature. ${ }^{479 b}$ Aggregated $A \beta$ decreases membrane fluidity, but $A \beta$ 
monomer does not. Aggregation rate and surface hydrophobicity were greater for A $\beta 40$ prepared at $\mathrm{pH} 6$ compared to $\mathrm{pH} 7 .{ }^{491}$ However, Wood et al. observe that $\mathrm{A} \beta 40$ does not form amyloid fibrils at $\mathrm{pH} 5.8$ (approximate $\mathrm{pH}$ of endosomes and conditions for proteolytic cleavage), but rather forms larger aggregates which lead to turbidity of the solution. ${ }^{422}$ Inhibition of $A \beta$ fibril formation was observed to depend on the state of the lipid membrane in studies of the interaction of $A \beta(M 1-40)$ and A $\beta$ (M1-42) (recombinantly expressed in E. Coli, Section 2.2.2) with liposomes. ${ }^{499}$ The largest retardation is observed when DPPC bilayers are in the solid gel phase. The mobility of $\mathrm{A} \beta 42$ prefibrillar and fibrillar oligomers on the membrane of living cells or lipid membranes has been investigated via single particle tracking techniques (using quantum dots as labels). ${ }^{500}$ The dynamic behaviour is distinct depending on the aggregation state (and conformation) of the peptide, although motion for oligomers is largely confined. Diffusion coefficients were obtained and differ significantly for fibrillar and prefibillar oligomers recognized by the A11 antibody. ${ }^{500}$

The insertion of the transmembrane fragment $A \beta(25-35)$ into phospholipid bilayers has been examined by neutron diffraction using deuterium labeling techniques. ${ }^{501}$ The location of the peptide $\mathrm{C}$ terminus was studied in two different lipid compositions and was found to be dependent on the surface charge of the membrane (zwitterionic or anionic). The adsorption of $A \beta 40$ on phospholipid monolayers has been probed using grazing incidence $\mathrm{x}$-ray diffraction and IRRAS. ${ }^{502}$ On negatively charged monolayers, the peptide adsorbs at the air-water interface and inserts into the monolayer, although it is squeezed out at high surface pressure. $A \beta 40$ can also penetrate into disordered anionic monolayers in buffer due to salt screening of electrostatic interactions. ${ }^{502}$ The influence of $A \beta(25-35)$ and $A \beta(22-40)$ on the picosecond dynamics of lipid 
membranes has been examined by quasi-elastic neutron scattering. ${ }^{503}$ The main influence was on long-range translational diffusion, although localized diffusion was also considered. By selection of the $A \beta(11-22)$ fragment, the role of charge, and the influence of $\mathrm{pH}$, in governing the interaction of $\mathrm{A} \beta$ with lipids was examined. ${ }^{504}$ Only at endosomal $\mathrm{pH}$ (approximately 6) does the peptide insert into negatively charged membranes, with a conformation change (increase in $\alpha$-helix content in the presence of lipids) detected by CD. Specifically, the protonation state of H13 and H14 was found to be important (as in the case of interactions with metal ions, section 4.8). ${ }^{504}$

Aggregation of $A \beta 42$ proceeds with distinct aggregate morphology (and kinetics) when adsorbed on hydrophilic mica or hydrophobic graphite. ${ }^{505}$ and this was related to fibrillization at interfaces such as membranes.

The role of cholesterol in $\mathrm{AD}$ has been examined due to the mutal interaction of $\mathrm{A} \beta$ and cholesterol (the metabolism of which is modulated by ApoE). ${ }^{472 a, 474 c, 477 a, 506}$ Cholesterol mediates $A \beta$ aggregation and reciprocally $A \beta$ influences cholesterol dynamics in neurons, leading to tauopathy. ${ }^{472 a}$ Whether cholesterol inhibits or promotes $A \beta$ fibrillization may depend on its content within the membrane, ${ }^{224 \mathrm{~g}, 507}$ and the presence of metal ions. ${ }^{508}$ Serum and CSF levels of cholesterol may provide a biomarker for $\mathrm{AD}$ (others are discussed in Section 2.4) although the relationship between HDL (high density lipoprotein) level and AD is not clearly established at present. ${ }^{472 a}$ A correlation between cholesterol (uncharged) content in the model membranes and $\mathrm{A} \beta 42$ deposition was noted, due to the effect of cholesterol on membrane rigidity (addition of $A \beta 40$ increased vesicle rigidity). ${ }^{496 a}$ The structure of lipid membranes extracted from AD patient brain tissue has been compared to control, 
using SAXS to obtain electron density profiles and differences were ascribed to the increase in cholesterol level in the AD brain. ${ }^{509}$ The changes in membrane rigidity in turn influence $\mathrm{Ca}^{2+}$ ion transport across neuronal cell membranes. ${ }^{485}$ An inverse correlation between membrane cholesterol level and $A \beta$-cell surface binding and cytotoxicity was observed. ${ }^{496 a}$ The role of cholesterol in amyloid aggregation and tau phosphorylation has been reviewed. ${ }^{472,510}$ Statins may have a role in alleviating AD pathologies associated with cholesterol since they can influence cholesterol metabolism in the human brain. ${ }^{472 a, 506,511}$ Several cholesterol derivatives have been found to enhance $A \beta 42$ fibrillization, and aspirin can inhibit this cholesterol-mediated fibrillization. ${ }^{512}$ The formation of the GM1 ganglioside-bound $\mathrm{A} \beta$ (GM1/A $\beta$ ) complex (found in the brains of $\mathrm{AD}$ patients and proposed to seed $\mathrm{A} \beta$ aggregation) is cholesterol-dependent. ${ }^{513}$ Cholesterol byproducts from antibody-induced ozonolysis during inflammation have been detected in human brains. ${ }^{514}$ These compounds which contain aldehydes dramatically accelerate $\mathrm{A} \beta$ aggregation in vitro. The authors note that these observations relate to some common features of $\mathrm{AD}$ and atherosclerosis, in particular in terms of inflammation. ${ }^{514}$ The mechanism of interaction of these cholesterol metabolites with $\mathrm{A} \beta 40$ was subsequently analysed in detail. ${ }^{515}$

\subsection{Effect of Metal Ions}

Aggregation of $A \beta$ may be promoted by metal ions. ${ }^{16 d, 516}$ High concentrations of metal ions $\left(\mathrm{Cu}^{2+}, \mathrm{Fe}^{3+}, \mathrm{Zn}^{2+}, \mathrm{Al}^{3+} \ldots\right)$ are found to be co-localized at abnormally high concentration with senile plaques in $\mathrm{AD}$ brains. ${ }^{517} \mathrm{~A} \beta$ rapidly aggregates in the presence of physiological concentrations of $\mathrm{Zn}^{2+}$ at $\mathrm{pH} 7.4 .^{106 \mathrm{~b}, 518}$ Enhanced $\mathrm{Cu}^{2+}$ induced aggregation is noted when the $\mathrm{pH}$ is lowered to $6.8 .^{518 \mathrm{~b}}$ Mildly acidic 
conditions are often associated with inflammation. APP binds copper in the $\mathrm{N}$ terminal domain within the $\operatorname{APP}(135-175)$ sequence. ${ }^{16 \mathrm{~d}}$ The copper binding domain contains a His-X-His motif. ${ }^{16 \mathrm{~d}}$ Metal ions are involved in processes associated with inflammation in $\mathrm{AD}$ patients (vide infra). Treatment with metal ion chelators can reduce the deposition of $A \beta$ in brains, as discussed further in section 3.2.1. ${ }^{242 \mathrm{a}, 242 \mathrm{~d}, 259}$ The focus of most studies has been $\mathrm{Cu}^{2+}, \mathrm{Zn}^{2+} \mathrm{Al}^{3+}$ and $\mathrm{Fe}^{3+}$, which enhance fibril formation.

Transition metal ions may interfere with transport across ion channels, eg. $\mathrm{Al}^{3+}$ ions ${ }^{225 a}$ and $\mathrm{Zn}^{2+}$ ions ${ }^{225 d, 226 a, 229,266 b}$ have been shown to block these channels. In the latter case, this can be reversed using a $\mathrm{Zn}^{2+}$ chelator. ${ }^{225 \mathrm{~d}}$ Arispe et al. also point to the formation of channels in bilayer membranes as a possible tool to screen for possible therapeutic compounds. ${ }^{225 a} \mathrm{Zn}^{2+}$ and $\mathrm{Cu}^{2+}$ ions induce $\mathrm{A} \beta 42$ and $\mathrm{A} \beta 40$ insertion into vesicles in a suitable $\mathrm{pH}$ range with an accompanying formation of $\alpha$-helical structures. $^{508}$

Surface plasmon resonance (SPR) biosensing has been used to investigate the aggregation of $\mathrm{A} \beta 40$ in the presence of $\mathrm{Cu}^{2+}, \mathrm{Ca}^{2+}, \mathrm{Fe}^{2+}$ and $\mathrm{Fe}^{3+} \cdot{ }^{519}$ All ions promoted $\mathrm{A} \beta$ aggregation, but with different rate constants, that for $\mathrm{Cu}^{2+}$ being highest, although the aggregates were unstable. The chelator EDTA (ethylenediamine tetraacetic acid) can dissociate metal-ion induced $\mathrm{A} \beta$ aggregates. ${ }^{519}$ Rottkamp et al. showed that if $A \beta$ is pretreated with the iron chelator deferoxamine, neuronal toxicity is significantly reduced while conversely, incubation of $A \beta$ with excess free iron restores toxicity to original levels. ${ }^{520}$ 
The aggregation of human $A \beta$ is stimulated by the presence of zinc ions at sufficiently high concentration due to binding mediated by histidine. ${ }^{106 \mathrm{~b}}$ The importance of the $\mathrm{H} 13$ residue in $\mathrm{A} \beta$ in binding $\mathrm{Cu}^{2+}$ and $\mathrm{Zn}^{2+}$ in a $\mathrm{pH}$-dependent manner was highlighted (the other $\mathrm{N}$ terminal histidines H6 and H14 also play a role). ${ }^{259 b, 518 b, 521}$ The $\mathrm{Cu}^{2+}$ binding site can also involve D1 or E11. ${ }^{522} \mathrm{~A}$ solid state NMR study has recently elucidated the $\mathrm{Cu}^{2+}$ binding site of $\mathrm{A} \beta 40{ }^{523}$ It was reported that under physiological conditions, aluminium, iron, and zinc strongly promote $\mathrm{A} \beta$ aggregation (rate enhancement of 100-1,000-fold) whereas the other metal ions (including calcium, copper and sodium) studied do not. ${ }^{524}$ The aggregation of $A \beta$ induced by iron or aluminium ions is distinguished from that of $\mathrm{Zn}^{2+}$ by its rate, extent and $\mathrm{pH}-$ and temperature-dependence as probed via sedimentation experiments using ${ }^{125} \mathrm{I}-$ labelled $A \beta(1-40) .{ }^{524}$ It has been proposed that rapid formation of a pre-oligomeric peptide/metal/peptide complex follows binding of $\mathrm{Cu}^{2+}$ to $\mathrm{A} \beta$, leading to inhibition of oligomer formation, ${ }^{525}$ as observed at low $\mathrm{Cu}^{2+}$ concentration. ${ }^{526}$ Ascorbate-dependent hydroxyl radical generation, is inhibited by $\mathrm{A} \beta(1-16)$ or $\mathrm{A} \beta 42$ for $\mathrm{Cu}^{2+}$ or $\mathrm{Fe}^{3+}$. $221 \mathrm{e}$

AFM imaging directly illustrates the influence of copper and zinc ions on the aggregation of $A \beta 42$, preventing fibrillization even in trace amounts. ${ }^{527}$ Using immobilized $\mathrm{A} \beta$ seeds (Section 4.3.1), $\mathrm{Cu}^{2+}$ and $\mathrm{Zn}^{2+}$ at neutral $\mathrm{pH}$ are found to accelerate the deposition of $\mathrm{A} \beta 40$ and $\mathrm{A} \beta 42$ but produce amorphous aggregates whereas $\mathrm{Fe}^{3+}$ induces the formation of fibrils. ${ }^{418 \mathrm{~b}}$ The effects of mixtures of $\mathrm{Cu}^{2+}, \mathrm{Zn}^{2+}$ and $\mathrm{Fe}^{3+}$ ions on $\mathrm{A} \beta 42$ aggregation has also been examined via AFM using the same technique. ${ }^{528}$ 
The role of aluminium in the etiology of AD is controversial. ${ }^{516 \mathrm{~d}, 529}$ It was originally found to be associated with plaques in AD patients, in the form of aluminosilicates ${ }^{530}$ specifically associated with neurofibrillary tangles. ${ }^{529 b, 531}$ Aluminium has significantly higher cytotoxicity in complexes with $\mathrm{A} \beta 42$ than $\mathrm{Cu}^{2+}, \mathrm{Zn}^{2+}$ and $\mathrm{Fe}^{3+}$ and the aggregation properties of $\mathrm{A} \beta$ in the presence of $\mathrm{Al}^{3+}$ are also substantially different. ${ }^{532}$

The effect of metal ions on $A \beta$ aggregation has been correlated to oxidative stress. ${ }^{517 b, 520}$ Both iron and copper have high affinity for $A \beta$ and are reduced by it, with the subsequent production of hydrogen peroxide and oxidised $A \beta .{ }^{533}$ Iron has been implicated as a key species in oxidative stress, due to its involvement in the creation of free radicals (in particular hydroxyl radicals ${ }^{517 b}$ ) from $\mathrm{H}_{2} \mathrm{O}_{2}$ via the Fenton reaction. ${ }^{534}$ It has been suggested that iron enhances the toxicity of $A \beta$ by delaying the deposition of the peptide into well-defined fibrils. ${ }^{535}$ The cytotoxic effects of $A \beta$ can be attenuated by antioxidants and free radical scavengers such as vitamin E.

The zinc binding motif, strongly conserved among members of the APP family has been identified. ${ }^{536}$ APP can catalyze the reduction of $\mathrm{H}_{2} \mathrm{O}_{2}$ and ensuing oxidation of $\mathrm{Cu}^{+}$to $\mathrm{Cu}^{2+}$ in a peroxidative reaction in vitro, leading via a Fenton-type reaction to free radical formation. ${ }^{536 \mathrm{c}}$

\subsection{Conjugates of $A \beta$ with Polymers and Lipid Chains}

Conjugation of peptides to synthetic polymers such as PEG may lead to improved solubility, enhanced stability against dilution, reduced toxicity and 
immunogenicity. ${ }^{537}$ The solution self-assembly of peptide-containing copolymers has been reviewed. $^{538}$

In a pioneering series of papers, Meredith and coworkers have confirmed the formation of fibrils in aqueous solutions of PEG-peptide diblocks where the peptide block was based on the central hydrophobic domain $A \beta(10-35)$ of the $\beta$-amyloid peptide and the PEG block had a molar mass of $3000 \mathrm{~g} \mathrm{~mol}^{-1}{ }^{539}$ They found from SANS and TEM that the PEG forms a coating around the fibril, thus acting as a "steric stabilization" layer. The self-assembly in aqueous solution of PEG-peptides, with peptide sequences based on KLVFF $A \beta(16-20)$ motif has been investigated, with PEG molar mass in the range $1000-3000 \mathrm{~g} \mathrm{~mol}^{-1}$. Conjugates FFKLVFF-PEG ${ }^{540}$ and YYKLVFF-PEG ${ }^{541}$ form core-shell fibrils and aggregation into nematic and hexagonal columnar liquid crystal phases is observed at high concentration. Conjugate $\beta A \beta A K L V F F-P E G$ was used in studies of enymatic cleavage (using $\alpha$ chymotrypsin to cleave between the two phenylalanine residues). ${ }^{542}$ The conjugate forms spherical micelles which are degraded by the enzyme, releasing peptide $\beta A \beta A K L V F$ (which does not aggregate into amyloid).

The formation of amyloid-like structures at the air/water interface was reported for peptide amphiphiles (PAs) with a peptide sequence based on $\mathrm{A} \beta(31-35)$, i.e. IIGLM, attached to a $\mathrm{C}_{18}$ chain. ${ }^{543}$ Epifluorescence microscopy showed the formation of threadlike and needle-like aggregates. Yilin Wang's group have shown that $\mathrm{PA} \mathrm{C}_{12^{-}}$ $\mathrm{A} \beta(11-17)$ forms fibrils. ${ }^{544}$ The double tail analogue PAs $2 \mathrm{C}_{12}$-Lys-A $\beta(12-17)$ and $\mathrm{C}_{12}-\mathrm{A} \beta(11-17)-\mathrm{C}_{12}$ self-assemble into fibrils or twisted ribbons/tapes respectively, the latter dependent on $\mathrm{pH}^{545}$ 


\section{SUMMARY AND OUTLOOK}

This review has focussed on the biological and biophysical properties of the Amyloid $\beta(\mathrm{A} \beta)$ peptide and its role in Alzheimer's disease. The biological, biochemical and neurochemical characteristics of $\mathrm{AD}$, involving $\mathrm{A} \beta$ and APP have been discussed. Therapeutic treatments including those on the market, or those that have been or continue to be the subject of clinical trials have been described. Finally, aspects of the biophysical chemistry of $A \beta$ and $A \beta$ fragment peptides have been outlined, including various aspects of structure at the molecular and supra-molecular level, and aggregation mechanisms and kinetics.

It is now more than a century since Alzheimer identified the disease now named after him, and more than a quarter century since aggregation of amyloid beta peptide was first associated with the condition. As a major global healthcare challenge, there has been intense research activity in this period. This has led to deep insights into the causative agents, the current consensus being that $A \beta$ is the primary suspect.

However, the progression of $\mathrm{AD}$ is not straightforward, and other factors are almost certainly involved, including other proteins such as tau, but also influences such as oxidative stress. Further large-scale genetic and proteomic screening studies will almost certainly reveal other risk factors.

A number of compounds are available to amelioriate the early stage symptoms of $\mathrm{AD}$, however there is still no effective treatment that can halt or reverse progression into the debilitating late stage of the disease. Several approaches have led to compounds 
that have reached phase III clinical trials, some of which failed. However, given the complexity associated with treatment of brain disease and the fact that it involves a subtle target, i.e. a protein misfolding process, some setbacks along the road are probably inevitable. The problem of finding a selective therapeutic agent is challenging given the diversity of biochemical pathways involved in brain signalling and other neuronal growth and differentiation processes. Nonetheless, there is reason for optimism as clinical trials of a number of therapeutic agents continue. There has been some refocusing by big pharma in the brain (and central nervous system) disease field, but some significant players are still very active in $R \& D$ related to $A D$ therapies. Furthermore, governments internationally are increasingly recognising the magnitude of the problem of diseases of aging, especially $\mathrm{AD}$, and there is currently substantial investment in further research through various funding agencies and networks. Probably closer to realisation are effective diagnostic systems based on blood or plasma analysis, or brain scanning methods.

In terms of biophysical measurements, it has to be noted that $A \beta$ is a difficult peptide to work with, in the sense that its aggregation properties are highly sensitive to sequence, purity and preparation conditions. The latter include initial dispersal solvent, nature and concentration of the aqueous or buffer solutions, but also the effect of shear during mixing and potentially the nature of the surface of the vessel. Small differences in preparation conditions can lead to distinct polymorphs, which can propagate, as discussed in section 4.4.

Despite these caveats, there is now a great wealth of data on the fibrillization properties of $A \beta$, variants and fragments under defined conditions. These 
measurements provide a strong framework to underpin the ongoing biological research activity.

\section{Acknowledgements.}

IWH is the recipient of a Royal Society-Wolfson Research Merit Award. Research in the Hamley group on amyloid peptides is supported by EPSRC grants EP/F048114/1, EP/G026203/1 and EP/G067538/1. 


\section{References}

(1) (a) Selkoe, D. J. Physiol. Rev. 2001, 81, 741. (b) Selkoe, D. J. Ann. Intern. Med. 2004, 140, 627. (c) Shankar, G. M.; Walsh, D. M. Mol. Neurodegener. 2009, 4.

(2) (a) Goedert, M.; Spillantini, M. G. Science 2006, 314, 777. (b) Hamley , I. W. Angew. Chem., Int. Ed. Engl. 2007, 46, 8128. (c) Irvine, G. B.; El-Agnaf, O. M. A.; Shankar, G. M.; Walsh, D. M. Molecular Medicine 2008, 14, 451. (d) Glabe, C. G. Neurobiology of Aging 2006, 27, 570.

(3) Selkoe, D. J. Nature Medicine 2011, 17, 1060.

(4) (a) Roberson, E. D.; Scearce-Levie, K.; Palop, J. J.; Yan, F. R.; Cheng, I. H.; Wu, T.; Gerstein, H.; Yu, G. Q.; Mucke, L. Science 2007, 316, 750. (b) Vossel, K. A.; Zhang, K.; Brodbeck, J.; Daub, A. C.; Sharma, P.; Finkbeiner, S.; Cui, B. X.; Mucke, L. Science 2010, 330, 198.

(5) (a) Ueda, K.; Fukushima, H.; Masliah, E.; Xia, Y.; Iwai, A.; Yoshimoto, M.;

Otero, D. A. C.; Kondo, J.; Ihara, Y.; Saitoh, T. Proc. Natl. Acad. Sci. U. S. A. 1993, 90, 11282. (b) Bodles, A. M.; Guthrie, D. J. S.; Greer, B.; Irvine, G. B. J. Neurochem. 2001, 78, 384.

(6) (a) Harper, J. D.; Lansbury, P. T. Annu. Rev. Biochem. 1997, 66, 385. (b) Teplow, D. B. Amyloid: Int. J. Exp. Clin. Invest. 1998, 5, 121. (c) Howlett, D. R. In Bioimaging in Neuroscience; Broderick, P. A., Rahni, D. N., Kolodny, E. H., Eds.; Humana: Totowa, NJ, 2005, p 61. (d) Lansbury, P. T.; Lashuel, H. A. Nature 2006, 443, 774. (e) Haass, C.; Selkoe, D. J. Nature Reviews Molecular Cell Biology 2007, 8, 101. (f) DeToma, A. S.; Salamekh, S.; Ramamoorthy, A.; Lim, M. H. Chem. Soc. Rev. 2012, 41, 608.

(7) Abbott, A. Nature 2011, 475, S2. 
(8) Mount, C.; Downton, C. Nature Medicine 2006, 12, 780.

(9) Luengo-Fernandez, R.; Leal, J.; Gray, A. モ Dementia 2010,ヤ Health Economics Research Centre, 2010.

(10) Tanzi, R. E. In Scientific American Molecular Neurology; Martin, J. B., Ed.; Scientific American: New York, 1998.

(11) Gandy, S. Nature 2011, 475, S15.

(12) (a) Mucke, L. Nature (London) 2009, 461, 895. (b) Deweerdt, S. Nature 2011, 475, S16.

(13) Halagappa, V. K. M.; Guo, Z. H.; Pearson, M.; Matsuoka, Y.; Cutler, R. G.; LaFerla, F. M.; Mattson, M. P. Neurobiol. Dis. 2007, 26, 212.

(14) (a) Melnikova, I. Nature Rev. Drug Disc. 2007, 6, 341. (b) Sabbagh, M. N. American Journal of Geriatric Pharmacotherapy 2009, 7, 167.

(15) Hsia, A. Y.; Masliah, E.; McConlogue, L.; Yu, G. Q.; Tatsuno, G.; Hu, K.; Kholodenko, D.; Malenka, R. C.; Nicoll, R. A.; Mucke, L. Proc. Natl. Acad. Sci. U. S. A. 1999, 96, 3228.

(16) (a) Evans, D. A.; Funkenstein, H.; Albert, M. S.; Scherr, P. A.; Cook, N. R.; Chown, M. J.; Hebert, L. E.; Hennekens, C. H.; Taylor, J. O. J. Am. Med. Assoc. 1989, 262, 2551. (b) Kukull, W. A.; Bowen, J. D. Med. Clin. N. Am. 2002, 86, 573. (c) Kukull, W. A.; Higdon, R.; Bowen, J. D.; McCormick, W. C.; Teri, L.; Schellenberg, G. D.; van Belle, G.; Jolley, L.; Larson, E. B. Arch. Neurol. 2002, 59, 1737. (d) Brown, D. R. Dalton Trans. 2009, 21, 4069.

(17) Markesbery, W. R. Free Radical Biology and Medicine 1997, 23, 134.

(18) Akiyama, H.; Barger, S.; Barnum, S.; Bradt, B.; Bauer, J.; Cole, G. M.; Cooper, N. R.; Eikelenboom, P.; Emmerling, M.; Fiebich, B. L.; Finch, C. E.; Frautschy, S.; Griffin, W. S. T.; Hampel, H.; Hull, M.; Landreth, G.; Lue, L. 
F.; Mrak, R.; Mackenzie, I. R.; McGeer, P. L.; O'Banion, M. K.; Pachter, J.; Pasinetti, G.; Plata-Salaman, C.; Rogers, J.; Rydel, R.; Shen, Y.; Streit, W.; Strohmeyer, R.; Tooyoma, I.; Van Muiswinkel, F. L.; Veerhuis, R.; Walker, D.; Webster, S.; Wegrzyniak, B.; Wenk, G.; Wyss-Coray, T. Neurobiology of Aging 2000, 21, 383.

(19) Hansson, O.; Zetterberg, H.; Buchhave, P.; Londos, E.; Blennow, K.; Minthon, L. Lancet Neurol. 2006, 5, 228.

(20) Sperling, R. A.; Aisen, P. S.; Beckett, L. A.; Bennett, D. A.; Craft, S.; Fagan, A. M.; Iwatsubo, T.; Jack, C. R.; Kaye, J.; Montine, T. J.; Park, D. C.; Reiman, E. M.; Rowe, C. C.; Siemers, E.; Stern, Y.; Yaffe, K.; Carrillo, M. C.; Thies, B.; Morrison-Bogorad, M.; Wagster, M. V.; Phelps, C. H. Alzheimers. Dement. 2011, 7, 280.

(21) Bishop, N. A.; Lu, T.; Yankner, B. A. Nature 2010, 464, 529.

(22) Fischer, D. F.; van Dijk, R.; van Tijn, P.; Hobo, B.; Verhage, M. C.; van der Schors, R. C.; Li, K. W.; van Minnen, J.; Hol, E. M.; van Leeuwen, F. W. Neurobiology of Aging 2009, 30, 847.

(23) Hardy, J.; Selkoe, D. J. Science 2002, 297, 353.

(24) Dickson, D. W. J. Neuropath. Exp. Neurol. 1997, 56, 321.

(25) (a) Roher, A. E.; Palmer, K. C.; Yurewicz, E. C.; Ball, M. J.; Greenberg, B. D. J. Neurochem. 1993, 61, 1916. (b) McLean, C. A.; Cherny, R. A.; Fraser, F. W.; Fuller, S. J.; Smith, M. J.; Beyreuther, K.; Bush, A. I.; Masters, C. L. Ann. Neurol. 1999, 46, 860. (c) Lue, L. F.; Kuo, Y. M.; Roher, A. E.; Brachova, L.; Shen, Y.; Sue, L.; Beach, T.; Kurth, J. H.; Rydel, R. E.; Rogers, J. Am. J. Pathol. 1999, 155, 853. (d) Näslund, J.; Haroutunian, V.; Mohs, R.; Davis, K. 
L.; Davies, P.; Greengard, P.; Buxbaum, J. D. J. Am. Med. Assoc. 2000, 283, 1571 .

(26) Blennow, K.; Hampel, H.; Weiner, M.; Zetterberg, H. Nat. Rev. Neurol. 2010, 6, 131.

(27) (a) Eanes, E. D.; Glenner, G. G. J. Histochem. Cytochem. 1968, 16, 673. (b) Kirschner, D. A.; Abraham, C.; Selkoe, D. J. Proc. Natl. Acad. Sci. U. S. A. 1986, 83, 503. (c) Sunde, M.; Blake, C. C. F. Adv. Protein Chem. 1997, 50, 123. (d) Serpell, L. C. Biochim. Biophys. Acta 2000, 1502, 16. (e) Makin, O. S.; Serpell, L. C. FEBS J 2005, 272, 5950.

(28) Walsh, D. M.; Thulin, E.; Minogue, A. M.; Gustavsson, N.; Pang, E.; Teplow, D. B.; Linse, S. FEBS J 2009, 276, 1266.

(29) Selkoe, D. J. Nature Medicine 2011, 17, 1521.

(30) Schnabel, J. Nature 2011, 475, S12.

(31) Roberson, E. D.; Mucke, L. Science 2006, 314, 781.

(32) Glenner, G. G.; Wong, C. W. Biochem. Biophys. Res. Commun. 1984, 120, 885.

(33) (a) Masters, C. L.; Simms, G.; Weinman, N. A.; Multhaup, G.; McDonald, B. L.; Beyreuther, K. Proc. Natl. Acad. Sci. U. S. A. 1985, 82, 4245. (b) Tanzi, R. E.; Gusella, J. F.; Watkins, P. C.; Bruns, G. A. P.; Stgeorgehyslop, P.; Vankeuren, M. L.; Patterson, D.; Pagan, S.; Kurnit, D. M.; Neve, R. L. Science 1987, 235, 880. (c) Kang, J.; Lemaire, H. G.; Unterbeck, A.; Salbaum, J. M.; Masters, C. L.; Grzeschik, K. H.; Multhaup, G.; Beyreuther, K.; Mullerhill, B. Nature 1987, 325, 733. (d) Goldgaber, D.; Lerman, M. I.; McBride, O. W.; Saffiotti, U.; Gajdusek, D. C. Science 1987, 235, 877. (e) Robakis, N. K.; Ramakrishna, N.; Wolfe, G.; Wisniewski, H. M. Proc. Natl. Acad. Sci. U. S. A. 1987, 84, 4190. 
(34) Jarrett, J. T.; Berger, E. P.; Lansbury, P. T. Biochemistry 1993, 32, 4693.

(35) (a) Qi-Takahara, Y.; Morishima-Kawashima, M.; Tanimura, Y.; Dolios, G.;

Hirotani, N.; Horikoshi, Y.; Kametani, F.; Maeda, M.; Saido, T. C.; Wang, R.; Ihara, Y. J. Neurosci. 2005, 25, 436. (b) Welander, H.; Franberg, J.; Graff, C.; Sundstrom, E.; Winblad, B.; Tjernberg, L. O. J. Neurochem. 2009, 110, 697.

(36) Roher, A. E.; Lowenson, J. D.; Clarke, S.; Wolkow, C.; Wang, R.; Cotter, R. J.; Reardon, I. M.; Zurcherneely, H. A.; Heinrikson, R. L.; Ball, M. J.; Greenberg, B. D. J. Biol. Chem. 1993, 268, 3072.

(37) Ida, N.; Hartmann, T.; Pantel, J.; Schroder, J.; Zerfass, R.; Forstl, H.; Sandbrink, R.; Masters, C. L.; Beyreuther, K. J. Biol. Chem. 1996, 271, 22908.

(38) Roher, A. E.; Lowenson, J. D.; Clarke, S.; Woods, A. S.; Cotter, R. J.; Gowing, E.; Ball, M. J. Proc. Natl. Acad. Sci. U. S. A. 1993, 90, 10836.

(39) (a) Bucciantini, M.; Giannoni, E.; Chiti, F.; Baroni, F.; Formigli, L.; Zurdo, J.; Taddei, N.; Ramponi, G.; Dobson, C. M.; Stefani, M. Nature 2002, 416, 507. (b) Dobson, C. M. Trends Biochem. Sci. 1999, 24, 329. (c) Bucciantini, M.; Calloni, G.; Chiti, F.; Formigli, L.; Nosi, D.; Dobson, C. M.; Stefani, M. J. Biol. Chem. 2004, 279, 31374.

(40) Walsh, D. M.; Tseng, B. P.; Rydel, R. E.; Podlisny, M. B.; Selkoe, D. J. Biochemistry 2000, 39, 10831.

(41) LaFerla, F. M.; Green, K. N.; Oddo, S. Nat. Rev. Neurosci. 2007, 8, 499.

(42) (a) Kokmen, E.; Whisnant, J. P.; Ofallon, W. M.; Chu, C. P.; Beard, C. M. Neurology 1996, 46, 154. (b) Moroney, J. T.; Bagiella, E.; Desmond, D. W.; Paik, M. C.; Stern, Y.; Tatemichi, T. K. Stroke 1996, 27, 1283. (c) Tatemichi, T. K.; Paik, M.; Bagiella, E.; Desmond, D. W.; Stern, Y.; Sano, M.; Hauser, 
W. A.; Mayeux, R. Neurology 1994, 44, 1885. (d) Kalaria, R. N. Neurobiology of Aging 2000, 21, 321.

(43) (a) Abe, K.; Tanzi, R. E.; Kogure, K. Neurosci. Lett. 1991, 125, 172. (b) Kalaria, R. N.; Bhatti, S. U.; Palatinsky, E. A.; Pennington, D. H.; Shelton, E. R.; Chan, H. W.; Perry, G.; Lust, W. D. Neuroreport 1993, 4, 211. (c) Kogure, K.; Kato, H. Stroke 1993, 24, 2121. (d) Koistinaho, J.; Pyykonen, I.; Keinanen, R.; Hokfelt, T. Neuroreport 1996, 7, 2727. (e) Yokota, M.; Saido, T. C.; Tani, E.; Yamaura, I.; Minami, N. J. Cereb. Blood Flow Metab. 1996, 16, 1219. (f) Jendroska, K.; Hoffmann, O. M.; Patt, S. Ann. N. Y. Acad. Sci. 1997, 826, 401. (g) Marshall, A. J.; Rattray, M.; Vaughan, P. F. T. Brain Res. 2006, 1099, 18. (44) Brody, D. L.; Magnoni, S.; Schwetye, K. E.; Spinner, M. L.; Esparza, T. J.; Stocchetti, N.; Zipfel, G. J.; Holtzman, D. M. Science 2008, 321, 1221.

(45) De Strooper, B.; Saftig, P.; Craessaerts, K.; Vanderstichele, H.; Guhde, G.; Annaert, W.; Von Figura, K.; Van Leuven, F. Nature 1998, 391, 387.

(46) Phiel, C. J.; Wilson, C. A.; Lee, V. M. Y.; Klein, P. S. Nature 2003, 423, 435.

(47) (a) Schmechel, D. E.; Saunders, A. M.; Strittmatter, W. J.; Crain, B. J.; Hulette, C. M.; Joo, S. H.; Pericakvance, M. A.; Goldgaber, D.; Roses, A. D. Proc. Natl. Acad. Sci. U. S. A. 1993, 90, 9649. (b) Saunders, A. M.; Strittmatter, W. J.; Schmechel, D.; Georgehyslop, P. H. S.; Pericakvance, M. A.; Joo, S. H.; Rosi, B. L.; Gusella, J. F.; Crappermaclachlan, D. R.; Alberts, M. J.; Hulette, C.; Crain, B.; Goldgaber, D.; Roses, A. D. Neurology 1993, 43, 1467. (c) Corder, E. H.; Saunders, A. M.; Strittmatter, W. J.; Schmechel, D. E.; Gaskell, P. C.; Small, G. W.; Roses, A. D.; Haines, J. L.; Pericakvance, M. A. Science 1993, 261, 921. (d) Bales, K. R.; Verina, T.; Dodel, R. C.; Du, Y. S.; Altstiel, 
L.; Bender, M.; Hyslop, P.; Johnstone, E. M.; Little, S. P.; Cummins, D. J.; Piccardo, P.; Ghetti, B.; Paul, S. M. Nature Genetics 1997, 17, 263.

(48) (a) Strittmatter, W. J.; Weisgraber, K. H.; Huang, D. Y.; Dong, L. M.; Salvesen, G. S.; Pericakvance, M.; Schmechel, D.; Saunders, A. M.; Goldgaber, D.; Roses, A. D. Proc. Natl. Acad. Sci. U. S. A. 1993, 90, 8098. (b) Bales, K. R.; Liu, F.; Wu, S.; Lin, S. Z.; Koger, D.; DeLong, C.; Hansen, J. C.; Sullivan, P. M.; Paul, S. M. J. Neurosci. 2009, 29, 6771.

(49) Selkoe, D. J. Nature Medicine 2011, 17, 1693.

(50) Hayden, E. C. Nature 2008, 455, 1155.

(51) Brodbeck, J.; Balestra, M. E.; Saunders, A. M.; Roses, A. D.; Mahley, R. W.; Huang, Y. Proc. Natl. Acad. Sci. U. S. A. 2008, 105, 1343.

(52) Goate, A.; Chartierharlin, M. C.; Mullan, M.; Brown, J.; Crawford, F.; Fidani, L.; Giuffra, L.; Haynes, A.; Irving, N.; James, L.; Mant, R.; Newton, P.; Rooke, K.; Roques, P.; Talbot, C.; Pericakvance, M.; Roses, A.; Williamson, R.; Rossor, M.; Owen, M.; Hardy, J. Nature 1991, 349, 704.

(53) (a) Sherrington, R.; Rogaev, E. I.; Liang, Y.; Rogaeva, E. A.; Levesque, G.; Ikeda, M.; Chi, H.; Lin, C.; Li, G.; Holman, K.; Tsuda, T.; Mar, L.; Foncin, J. F.; Bruni, A. C.; Montesi, M. P.; Sorbi, S.; Rainero, I.; Pinessi, L.; Nee, L.; Chumakov, I.; Pollen, D.; Brookes, A.; Sanseau, P.; Polinsky, R. J.; Wasco, W.; Dasilva, H. A. R.; Haines, J. L.; Pericakvance, M. A.; Tanzi, R. E.; Roses, A. D.; Fraser, P. E.; Rommens, J. M.; St George-Hyslop, P. H. Nature 1995, 375, 754. (b) Rogaev, E. I.; Sherrington, R.; Rogaeva, E. A.; Levesque, G.; Ikeda, M.; Liang, Y.; Chi, H.; Lin, C.; Holman, K.; Tsuda, T.; Mar, L.; Sorbi, S.; Nacmias, B.; Piacentini, S.; Amaducci, L.; Chumakov, I.; Cohen, D.; 
Lannfelt, L.; Fraser, P. E.; Rommens, J. M.; St George-Hyslop, P. H. Nature 1995, 376,775 .

(54) Lambert, J. C.; Heath, S.; Even, G.; Campion, D.; Sleegers, K.; Hiltunen, M.;

Combarros, O.; Zelenika, D.; Bullido, M. J.; Tavernier, B.; Letenneur, L.;

Bettens, K.; Berr, C.; Pasquier, F.; Fievet, N.; Barberger-Gateau, P.;

Engelborghs, S.; De Deyn, P.; Mateo, I.; Franck, A.; Helisalmi, S.; Porcellini, E.; Hanon, O.; de Pancorbo, M. M.; Lendon, C.; Dufouil, C.; Jaillard, C.; Leveillard, T.; Alvarez, V.; Bosco, P.; Mancuso, M.; Panza, F.; Nacmias, B.; Bossu, P.; Piccardi, P.; Annoni, G.; Seripa, D.; Galimberti, D.; Hannequin, D.; Licastro, F.; Soininen, H.; Ritchie, K.; Blanche, H.; Dartigues, J. F.; Tzourio, C.; Gut, I.; Van Broeckhoven, C.; Alperovitch, A.; Lathrop, M.; Amouyel, P. Nature Genetics 2009, 41, 1094.

(55) (a) Russo, C.; Schettini, G.; Saido, T. C.; Hulette, C.; Lippall, C.; Lannfelt, L.; Ghetti, B.; Gambetti, P.; Tabaton, M.; Teller, J. K. Nature 2000, 405, 531. (b) Yoo, A. S.; Cheng, I.; Chung, S.; Grenfell, T. Z.; Lee, H.; Pack-Chung, E.; Handler, M.; Shen, J.; Xia, W. M.; Tesco, G.; Saunders, A. J.; Ding, K.; Frosch, M. P.; Tanzi, R. E.; Kim, T. W. Neuron 2000, 27, 561.

(56) Scheuner, D.; Eckman, C.; Jensen, M.; Song, X.; Citron, M.; Suzuki, N.; Bird, T. D.; Hardy, J.; Hutton, M.; Kukull, W.; Larson, E.; LevyLahad, E.; Viitanen, M.; Peskind, E.; Poorkaj, P.; Schellenberg, G.; Tanzi, R.; Wasco, W.; Lannfelt, L.; Selkoe, D.; Younkin, S. Nature Medicine 1996, 2, 864.

(57) (a) Borchelt, D. R.; Thinakaran, G.; Eckman, C. B.; Lee, M. K.; Davenport, F.; Ratovitsky, T.; Prada, C. M.; Kim, G.; Seekins, S.; Yager, D.; Slunt, H. H.; Wang, R.; Seeger, M.; Levey, A. I.; Gandy, S. E.; Copeland, N. G.; Jenkins, N. A.; Price, D. L.; Younkin, S. G. Neuron 1996, 17, 1005. (b) Duff, K.; 
Eckman, C.; Zehr, C.; Yu, X.; Prada, C. M.; Pereztur, J.; Hutton, M.; Buee, L.; Harigaya, Y.; Yager, D.; Morgan, D.; Gordon, M. N.; Holcomb, L.; Refolo, L.; Zenk, B.; Hardy, J.; Younkin, S. Nature 1996, 383, 710. (c) Citron, M.; Westaway, D.; Xia, W. M.; Carlson, G.; Diehl, T.; Levesque, G.; JohnsonWood, K.; Lee, M.; Seubert, P.; Davis, A.; Kholodenko, D.; Motter, R.; Sherrington, R.; Perry, B.; Yao, H.; Strome, R.; Lieberburg, I.; Rommens, J.; Kim, S.; Schenk, D.; Fraser, P.; Hyslop, P. S.; Selkoe, D. J. Nature Medicine 1997, 3, 67. (d) Tomita, T.; Maruyama, K.; Saido, T. C.; Kume, H.; Shinozaki, K.; Tokuhiro, S.; Capell, A.; Walter, J.; Grunberg, J.; Haass, C.; Iwatsubo, T.; Obata, K. Proc. Natl. Acad. Sci. U. S. A. 1997, 94, 2025. (e) Oyama, F.; Sawamura, N.; Kobayashi, K.; Morishima-Kawashima, M.; Kuramochi, T.; Ito, M.; Tomita, T.; Maruyama, K.; Saido, T. C.; Iwatsubo, T.; Capell, A.; Walter, J.; Grunberg, L.; Ueyama, Y.; Haass, C.; Ihara, Y. J. Neurochem. 1998, 71, 313.

(58) (a) Smith, I. F.; Green, K. N.; LaFerla, F. M. Cell Calcium 2005, 38, 427. (b) Tu, H. P.; Nelson, O.; Bezprozvanny, A.; Wang, Z. N.; Lee, S. F.; Hao, Y. H.; Serneels, L.; De Strooper, B.; Yu, G.; Bezprozvanny, I. Cell 2006, 126, 981. (c) Zhang, H.; Sun, S. Y.; Herreman, A.; De Strooper, B.; Bezprozvanny, I. J. Neurosci. 2010, 30, 8566.

(59) Cheung, K. H.; Shineman, D.; Muller, M.; Cardenas, C.; Mei, L. J.; Yang, J.; Tomita, T.; Iwatsubo, T.; Lee, V. M. Y.; Foskett, J. K. Neuron 2008, 58, 871. (60) Holcomb, L.; Gordon, M. N.; McGowan, E.; Yu, X.; Benkovic, S.; Jantzen, P.; Wright, K.; Saad, I.; Mueller, R.; Morgan, D.; Sanders, S.; Zehr, C.; O'Campo, K.; Hardy, J.; Prada, C. M.; Eckman, C.; Younkin, S.; Hsiao, K.; Duff, K. Nature Medicine 1998, 4, 97. 
(61) (a) Lemere, C. A.; Lopera, F.; Kosik, K. S.; Lendon, C. L.; Ossa, J.; Saido, T. C.; Yamaguchi, H.; Ruiz, A.; Martinez, A.; Madrigal, L.; Hincapie, L.; Arango, J. C. L.; Anthony, D. C.; Koo, E. H.; Goate, A. M.; Selkoe, D. J.; Arango, J. C. V. Nature Medicine 1996, 2, 1146. (b) Mann, D. M. A.; Iwatsubo, T.; Cairns, N. J.; Lantos, P. L.; Nochlin, D.; Sumi, S. M.; Bird, T. D.; Poorkaj, P.; Hardy, J.; Hutton, M.; Prihar, G.; Crook, R.; Rossor, M. N.; Haltia, M. Ann. Neurol. 1996, $40,149$.

(62) Wang, B. X.; Yang, W.; Wen, W.; Sun, J.; Su, B.; Liu, B.; Ma, D. L.; Lv, D.; Wen, Y. R.; Qu, T.; Chen, M.; Sun, M. A.; Shen, Y.; Zhang, X. Science 2010, $330,1065$.

(63) Castellano, J. M.; Kim, J.; Stewart, F. R.; Jiang, H.; DeMattos, R. B.; Patterson, B. W.; Fagan, A. M.; Morris, J. C.; Mawuenyega, K. G.; Cruchaga, C.; Goate, A. M.; Bales, K. R.; Paul, S. M.; Bateman, R. J.; Holtzman, D. M. Sci. Transl. Med. 2011, 3, 89ra57.

(64) Harold, D.; Abraham, R.; Hollingworth, P.; Sims, R.; Gerrish, A.; Hamshere, M. L.; Pahwa, J. S.; Moskvina, V.; Dowzell, K.; Williams, A.; Jones, N.; Thomas, C.; Stretton, A.; Morgan, A. R.; Lovestone, S.; Powell, J.; Proitsi, P.; Lupton, M. K.; Brayne, C.; Rubinsztein, D. C.; Gill, M.; Lawlor, B.; Lynch, A.; Morgan, K.; Brown, K. S.; Passmore, P. A.; Craig, D.; McGuinness, B.; Todd, S.; Holmes, C.; Mann, D.; Smith, A. D.; Love, S.; Kehoe, P. G.; Hardy, J.; Mead, S.; Fox, N.; Rossor, M.; Collinge, J.; Maier, W.; Jessen, F.; Schurmann, B.; van den Bussche, H.; Heuser, I.; Kornhuber, J.; Wiltfang, J.; Dichgans, M.; Frolich, L.; Hampel, H.; Hull, M.; Rujescu, D.; Goate, A. M.; Kauwe, J. S. K.; Cruchaga, C.; Nowotny, P.; Morris, J. C.; Mayo, K.; Sleegers, K.; Bettens, K.; Engelborghs, S.; De Deyn, P. P.; Van Broeckhoven, C.; Livingston, G.; Bass, 
N. J.; Gurling, H.; McQuillin, A.; Gwilliam, R.; Deloukas, P.; Al-Chalabi, A.;

Shaw, C. E.; Tsolaki, M.; Singleton, A. B.; Guerreiro, R.; Muhleisen, T. W.;

Nothen, M. M.; Moebus, S.; Jockel, K. H.; Klopp, N.; Wichmann, H. E.;

Carrasquillo, M. M.; Pankratz, V. S.; Younkin, S. G.; Holmans, P. A.;

O'Donovan, M.; Owen, M. J.; Williams, J. Nature Genetics 2009, 41, 1088.

(65) (a) Ghiso, J.; Matsubara, E.; Koudinov, A.; Choimiura, N. H.; Tomita, M.;

Wisniewski, T.; Frangione, B. Biochem. J. 1993, 293, 27. (b) Matsubara, E.;

Frangione, B.; Ghiso, J. J. Biol. Chem. 1995, 270, 7563.

(66) Calero, M.; Rostagno, A.; Matsubara, E.; Zlokovic, B.; Frangione, B.; Ghiso, J. Microsc. Res. Tech. 2000, 50, 305.

(67) (a) DeMattos, R. B.; Cirrito, J. R.; Parsadanian, M.; May, P. C.; O'Dell, M. A.; Taylor, J. W.; Harmony, J. A. K.; Aronow, B. J.; Bales, K. R.; Paul, S. M.;

Holtzman, D. M. Neuron 2004, 41, 193. (b) Bell, R. D.; Sagare, A. P.;

Friedman, A. E.; Bedi, G. S.; Holtzman, D. M.; Deane, R.; Zlokovic, B. V. J. Cereb. Blood Flow Metab. 2007, 27, 909.

(68) DeMattos, R. B.; O'Dell, M. A.; Parsadanian, M.; Taylor, J. W.; Harmony, J. A. K.; Bales, K. R.; Paul, S. M.; Aronow, B. J.; Holtzman, D. M. Proc. Natl. Acad. Sci. U. S. A. 2002, 99, 10843.

(69) Treusch, S.; Hamamichi, S.; Goodman, J. L.; Matlack, K. E. S.; Chung, C. Y.; Baru, V.; Shulman, J. M.; Parrado, A.; Bevis, B. J.; Valastyan, J. S.; Han, H.; Lindhagen-Persson, M.; Reiman, E. M.; Evans, D. A.; Bennett, D. A.; Olofsson, A.; DeJager, P. L.; Tanzi, R. E.; Caldwell, K. A.; Caldwell, G. A.; Lindquist, S. Science 2011, 334, 1241.

(70) Reiman, E. M.; Webster, J. A.; Myers, A. J.; Hardy, J.; Dunckley, T.; Zismann, V. L.; Joshipura, K. D.; Pearson, J. V.; Hu-Lince, D.; Huentelman, M. J.; 
Craig, D. W.; Coon, K. D.; Liang, W. S.; Herbert, R. H.; Beach, T.; Rohrer, K. C.; Zhao, A. S.; Leung, D.; Bryden, L.; Marlowe, L.; Kaleem, M.; Mastroeni, D.; Grover, A.; Heward, C. B.; Ravid, R.; Rogers, J.; Hutton, M. L.; Melquist, S.; Petersen, R. C.; Alexander, G. E.; Caselli, R. J.; Kukull, W.; Papassotiropoulos, A.; Stephan, D. A. Neuron 2007, 54, 713.

(71) Kalman, J.; McConathy, W.; Araoz, C.; Kasa, P.; Lacko, A. G. Neurol. Res. 2000, 22, 330 .

(72) Dreses-Werringloer, U.; Lambert, J. C.; Vingtdeux, V.; Zhao, H. T.; Vais, H.; Siebert, A.; Jain, A.; Koppel, J.; Rovelet-Lecrux, A.; Hannequin, D.; Pasquier, F.; Galimberti, D.; Scarpini, E.; Mann, D.; Lendon, C.; Campion, D.; Amouyel, P.; Davies, P.; Foskett, J. K.; Campagne, F.; Marambaud, P. Cell 2008, 133, 1149.

(73) Li, H.; Wetten, S.; Li, L.; Jean, P. L. S.; Upmanyu, R.; Surh, L.; Hosford, D.; Barnes, M. R.; Briley, J. D.; Borrie, M.; Coletta, N.; Delisle, R.; Dhalla, D.; Ehm, M. G.; Feldman, H. H.; Fornazzari, L.; Gauthier, S.; Goodgame, N.; Guzman, D.; Hammond, S.; Hollingworth, P.; Hsiung, G. Y.; Johnson, J.; Kelly, D. D.; Keren, R.; Kertesz, A.; King, K. S.; Lovestone, S.; Loy-English, I.; Matthews, P. M.; Owen, M. J.; Plumpton, M.; Pryse-Phillips, W.; Prinjha, R. K.; Richardson, J. C.; Saunders, A.; Slater, A. J.; George-Hyslop, P. H. S.; Stinnett, S. W.; Swartz, J. E.; Taylor, R. L.; Wherrett, J.; Williams, J.; Yarnall, D. P.; Gibson, R. A.; Irizarry, M. C.; Middleton, L. T.; Roses, A. D. Arch. Neurol. 2008, 65, 45.

(74) Bertram, L.; Schjeide, B. M. M.; Hooli, B.; Mullin, K.; Hiltunen, M.; Soininen, H.; Ingelsson, M.; Lannfelt, L.; Blacker, D.; Tanzi, R. E. Cell 2008, 135, 993. 
(75) Campagne, F.; Lambert, J. C.; Dreses-Werringloer, U.; Vingtdeux, V.; Lendon, C.; Campion, D.; Amouyel, P.; Lee, A. T.; Gregersen, P. K.; Davies, P.; Marambaud, P. Cell 2008, 135, 994.

(76) Thathiah, A.; Spittaels, K.; Hoffmann, M.; Staes, M.; Cohen, A.; Horre, K.; Vanbrabant, M.; Coun, F.; Baekelandt, V.; Delacourte, A.; Fischer, D. F.; Pollet, D.; De Strooper, B.; Merchiers, P. Science 2009, 323, 946.

(77) Blacker, D.; Bertram, L.; Saunders, A. J.; Moscarillo, T. J.; Albert, M. S.;

Wiener, H.; Perry, R. T.; Collins, J. S.; Harrell, L. E.; Go, R. C. P.; Mahoney, A.; Beaty, T.; Fallin, M. D.; Avramopoulos, D.; Chase, G. A.; Folstein, M. F.; McInnis, M. G.; Bassett, S. S.; Doheny, K. J.; Pugh, E. W.; Tanzi, R. E. Hum. Mol. Genet. 2003, 12, 23.

(78) Nitsch, R. M.; Deng, M. H.; Growdon, J. H.; Wurtman, R. J. J. Biol. Chem. 1996, 271,4188

(79) Pooler, A. M.; Arjona, A. A.; Lee, R. K.; Wurtman, R. J. Neurosci. Lett. 2004, $362,127$.

(80) Pasinetti, G. M.; Aisen, P. S. Neuroscience 1998, 87, 319.

(81) Ijsselstijn, L.; Dekker, L. J. M.; Stingl, C.; van der Weiden, M. M.; Hofman, A.; Kros, J. M.; Koudstaal, P. J.; Smitt, P.; Ikram, M. A.; Breteler, M. M. B.; Luider, T. M. J. Proteome Res. 2011, 10, 4902.

(82) (a) Cohen, E.; Paulsson, J. F.; Blinder, P.; Burstyn-Cohen, T.; Du, D. G.; Estepa, G.; Adame, A.; Pham, H. M.; Holzenberger, M.; Kelly, J. W.; Masliah, E.; Dillin, A. Cell 2009, 139, 1157. (b) Freude, S.; Hettich, M. M.; Schumann, C.; Stohr, O.; Koch, L.; Kohler, C.; Udelhoven, M.; Leeser, U.; Muller, M.; Kubota, N.; Kadowaki, T.; Krone, W.; Schroder, H.; Bruning, J. C.; Schubert, 
M. FASEB J. 2009, 23, 3315. (c) Moloney, A. M.; Griffin, R. J.; Timmons, S.;

O'Connor, R.; Ravid, R.; O'Neill, C. Neurobiology of Aging 2010, 31, 224.

(83) (a) Games, D.; Adams, D.; Alessandrini, R.; Barbour, R.; Berthelette, P.;

Blackwell, C.; Carr, T.; Clemens, J.; Donaldson, T.; Gillespie, F.; Guido, T.;

Hagopian, S.; Johnsonwood, K.; Khan, K.; Lee, M.; Leibowitz, P.;

Lieberburg, I.; Little, S.; Masliah, E.; McConlogue, L.; Montoyazavala, M.;

Mucke, L.; Paganini, L.; Penniman, E.; Power, M.; Schenk, D.; Seubert, P.;

Snyder, B.; Soriano, F.; Tan, H.; Vitale, J.; Wadsworth, S.; Wolozin, B.; Zhao,

J. Nature 1995, 373, 523. (b) Rockenstein, E. M.; McConlogue, L.; Tan, H.;

Power, M.; Masliah, E.; Mucke, L. J. Biol. Chem. 1995, 270, 28257. (c)

Schenk, D.; Barbour, R.; Dunn, W.; Gordon, G.; Grajeda, H.; Guido, T.; Hu,

K.; Huang, J. P.; Johnson-Wood, K.; Khan, K.; Kholodenko, D.; Lee, M.;

Liao, Z. M.; Lieberburg, I.; Motter, R.; Mutter, L.; Soriano, F.; Shopp, G.;

Vasquez, N.; Vandevert, C.; Walker, S.; Wogulis, M.; Yednock, T.; Games,

D.; Seubert, P. Nature 1999, 400, 173.

(84) (a) Janus, C.; Pearson, J.; McLaurin, J.; Mathews, P. M.; Jiang, Y.; Schmidt, S.

D.; Chishti, M. A.; Horne, P.; Heslin, D.; French, J.; Mount, H. T. J.; Nixon,

R. A.; Mercken, M.; Bergeron, C.; Fraser, P. E.; St George-Hyslop, P.;

Westaway, D. Nature 2000, 408, 979. (b) McLaurin, J.; Kierstead, M. E.;

Brown, M. E.; Hawkes, C. A.; Lambermon, M. H. L.; Phinney, A. L.; Darabie, A. A.; Cousins, J. E.; French, J. E.; Lan, M. F.; Chen, F. S.; Wong, S. S. N.;

Mount, H. T. J.; Fraser, P. E.; Westaway, D.; St George-Hyslop, P. Nature

Medicine 2006, 12, 801.

(85) Hsiao, K.; Chapman, P.; Nilsen, S.; Eckman, C.; Harigaya, Y.; Younkin, S.;

Yang, F. S.; Cole, G. Science 1996, 274, 99. 
(86) Wilcock, D. M.; DiCarlo, G.; Henderson, D.; Jackson, J.; Clarke, K.; Ugen, K. E.; Gordon, M. N.; Morgan, D. J. Neurosci. 2003, 23, 3745.

(87) Lesné, S.; Koh, M. T.; Kotilinek, L.; Kayed, R.; Glabe, C. G.; Yang, A.; Gallagher, M.; Ashe, K. H. Nature 2006, 440, 352.

(88) Oddo, S.; Caccamo, A.; Shepherd, J. D.; Murphy, M. P.; Golde, T. E.; Kayed, R.; Metherate, R.; Mattson, M. P.; Akbari, Y.; LaFerla, F. M. Neuron 2003, 39, 409.

(89) Meyer-Luehmann, M.; Coomaraswamy, J.; Bolmont, T.; Kaeser, S.; Schaefer, C.; Kilger, E.; Neuenschwander, A.; Abramowski, D.; Frey, P.; Jaton, A. L.; Vigouret, J. M.; Paganetti, P.; Walsh, D. M.; Mathews, P. M.; Ghiso, J.; Staufenbiel, M.; Walker, L. C.; Jucker, M. Science 2006, 313, 1781.

(90) (a) Burdick, D.; Soreghan, B.; Kwon, M.; Kosmoski, J.; Knauer, M.; Henschen, A.; Yates, J.; Cotman, C.; Glabe, C. J. Biol. Chem. 1992, 267, 546. (b) Jarrett, J. T.; Lansbury, P. T. Cell (Cambridge, Mass.) 1993, 73, 1055.

(91) (a) Dahlgren, K. N.; Manelli, A. M.; Stine, W. B.; Baker, L. K.; Krafft, G. A.; LaDu, M. J. J. Biol. Chem. 2002, 277, 32046. (b) Zhang, Y.; McLaughlin, R.; Goodyer, C.; LeBlanc, A. J. Cell Biol. 2002, 156, 519.

(92) (a) Citron, M.; Oltersdorf, T.; Haass, C.; McConlogue, L.; Hung, A. Y.; Seubert, P.; Vigopelfrey, C.; Lieberburg, I.; Selkoe, D. J. Nature 1992, 360, 672. (b) Cai, X. D.; Golde, T. E.; Younkin, S. G. Science 1993, 259, 514. (c) Suzuki, N.; Cheung, T. T.; Cai, X. D.; Odaka, A.; Otvos, L.; Eckman, C.; Golde, T. E.; Younkin, S. G. Science 1994, 264, 1336.

(93) Iwatsubo, T.; Odaka, A.; Suzuki, N.; Mizusawa, H.; Nukina, N.; Ihara, Y. Neuron 1994, 13, 45. 
(94) Gravina, S. A.; Ho, L. B.; Eckman, C. B.; Long, K. E.; Otvos, L.; Younkin, L. H.; Suzuki, N.; Younkin, S. G. J. Biol. Chem. 1995, 270, 7013.

(95) Soscia, S. J.; Kirby, J. E.; Washicosky, K. J.; Tucker, S. M.; Ingelsson, M.;

Hyman, B.; Burton, M. A.; Goldstein, L. E.; Duong, S.; Tanzi, R. E.; Moir, R. D. Plos One 2010, 5.

(96) Soscia, S. J.; Kirby, J. E.; Washicosky, K. J.; Tucker, S. M.; Ingelsson, M.; Hyman, B.; Burton, M. A.; Goldstein, L. E.; Duong, S.; Tanzi, R. E.; Moir, R. D. Plos One 2010, 5, e9505.

(97) Balin, B. J.; Little, C. S.; Hammond, C. J.; Appelt, D. M.; Whittum-Hudson, J. A.; Gerard, H. C.; Hudson, A. P. J. Alzheimers Dis. 2008, 13, 371.

(98) Appelt, D. M.; Roupas, M. R.; Way, D. S.; Bell, M. G.; Albert, E. V.; Hammond, C. J.; Balin, B. J. BMC Neuroscience 2008, 9, 13.

(99) Castellani, R. J.; Lee, H. G.; Siedlak, S. L.; Nunomura, A.; Hayashi, T.; Nakamura, M.; Zhu, X. W.; Perry, G.; Smith, M. A. J. Alzheimers Dis. 2009, $18,447$.

(100) (a) Yu, Y. W. Y.; Lin, C.-H.; Chen, S.-P.; Hong, C.-J.; Tsai, S.-J. Neurosci. Lett. 2000, 294, 179. (b) Han, S. D.; Drake, A. I.; Cessante, L. M.; Jak, A. J.; Houston, W. S.; Delis, D. C.; Filoteo, J. V.; Bondi, M. W. J. Neurol. Neurosurg. Psychiatry 2007, 78, 1103. (c) Marchant, N. L.; King, S. L.; Tabet, N.; Rusted, J. M. Neuropsychopharmacology 2010, 35, 1090.

(101) Mehta, P. D.; Pirttila, T.; Mehta, S. P.; Sersen, E. A.; Aisen, P. S.; Wisniewski, H. M. Arch. Neurol. 2000, 57, 100.

(102) Motter, R.; Vigopelfrey, C.; Kholodenko, D.; Barbour, R.; Johnsonwood, K.; Galasko, D.; Chang, L.; Miller, B.; Clark, C.; Green, R.; Olson, D.; 
Southwick, P.; Wolfert, R.; Munroe, B.; Lieberburg, I.; Seubert, P.; Schenk, D. Ann. Neurol. 1995, 38, 643.

(103) Demattos, R. B.; Bales, K. R.; Cummins, D. J.; Paul, S. M.; Holtzman, D. M. Science 2002, 295, 2264.

(104) Cleary, J. P.; Walsh, D. M.; Hofmeister, J. J.; Shankar, G. M.; Kuskowski, M. A.; Selkoe, D. J.; Ashe, K. H. Nature Neuroscience 2005, 8, 79.

(105) Podlisny, M. B.; Walsh, D. M.; Amarante, P.; Ostaszewski, B. L.; Stimson, E. R.; Maggio, J. E.; Teplow, D. B.; Selkoe, D. J. Biochemistry 1998, 37, 3602. (106) (a) Seubert, P.; Vigopelfrey, C.; Esch, F.; Lee, M.; Dovey, H.; Davis, D.; Sinha, S.; Schlossmacher, M.; Whaley, J.; Swindlehurst, C.; McCormack, R.; Wolfert, R.; Selkoe, D.; Lieberburg, I.; Schenk, D. Nature 1992, 359, 325. (b) Bush, A. I.; Pettingell, W. H.; Multhaup, G.; Paradis, M. D.; Vonsattel, J. P.; Gusella, J. F.; Beyreuther, K.; Masters, C. L.; Tanzi, R. E. Science 1994, 265, 1464.

(107) Mawuenyega, K. G.; Sigurdson, W.; Ovod, V.; Munsell, L.; Kasten, T.; Morris, J. C.; Yarasheski, K. E.; Bateman, R. J. Science 2010, 330, 1774.

(108) (a) Zlokovic, B. V. J. Neurochem. 2004, 89, 807. (b) Tanzi, R. E.; Moir, R. D.; Wagner, S. L. Neuron 2004, 43, 605.

(109) (a) Rosen, D. R.; Martinmorris, L.; Luo, L. Q.; White, K. Proc. Natl. Acad. Sci. U. S. A. 1989, 86, 2478. (b) Gunawardena, S.; Goldstein, L. S. B. Neuron 2001, 32, 389. (c) Iijima, K.; Liu, H. P.; Chiang, A. S.; Hearn, S. A.; Konsolaki, M.; Zhong, Y. Proc. Natl. Acad. Sci. U. S. A. 2004, 101, 6623. (d) Crowther, D. C.; Kinghorn, K. J.; Miranda, E.; Page, R.; Curry, J. A.; Duthie, F. A. I.; Gubb, D. C.; Lomas, D. A. Neuroscience 2005, 132, 123. (e) Nerelius, C.; Sandegren, A.; Sargsyan, H.; Raunak, R.; Leijonmarck, H.; 
Chatterjee, U.; Fisahn, A.; Imarisio, S.; Lomas, D. A.; Crowther, D. C.;

Stromberg, R.; Johansson, J. Proc. Natl. Acad. Sci. U. S. A. 2009, 106, 9191.

(f) Chakraborty, R.; Vepuri, V.; Mhatre, S. D.; Paddock, B. E.; Miller, S.;

Michelson, S. J.; Delvadia, R.; Desai, A.; Vinokur, M.; Melicharek, D. J.;

Utreja, S.; Khandelwal, P.; Ansaloni, S.; Goldstein, L. E.; Moir, R. D.; Lee, J.

C.; Tabb, L. P.; Saunders, A. J.; Marenda, D. R. Plos One 2011, 6. (g)

Luheshi, L. M.; Tartaglia, G. G.; Brorsson, A. C.; Pawar, A. P.; Watson, I. E.;

Chiti, F.; Vendruscolo, M.; Lomas, D. A.; Dobson, C. M.; Crowther, D. C.

Plos Biology 2007, 5, 2493.

(110) (a) Link, C. D. Proc. Natl. Acad. Sci. U. S. A. 1995, 92, 9368. (b) Yu, G.;

Nishimura, M.; Arawaka, S.; Levitan, D.; Zhang, L. L.; Tandon, A.; Song, Y.

Q.; Rogaeva, E.; Chen, F. S.; Kawaral, T.; Supala, A.; Levesque, L.; Yu, H.;

Yang, D. S.; Holmes, E.; Millman, P.; Liang, Y.; Zhang, D. M.; Xu, D. H.;

Sato, C.; Rogaev, E.; Smith, M.; Janus, C.; Zhang, Y. N.; Aebersold, R.;

Farrer, L.; Sorbi, S.; Bruni, A.; Fraser, P.; St George-Hyslop, P. Nature 2000,

407, 48. (c) Cohen, E.; Bieschke, J.; Perciavalle, R. M.; Kelly, J. W.; Dillin, A.

Science 2006, 313, 1604.

(111) Youm, J. W.; Kim, H.; Lo Han, J. H.; Jang, C. H.; Ha, H. J.; Mook-Jung, I.;

Jeon, J. H.; Choi, C. Y.; Kim, Y. H.; Kim, H. S.; Joung, H. Y. FEBS Lett. 2005, 579, 6737 .

(112) (a) Zhang, H. Y.; Komano, H.; Fuller, R. S.; Gandy, S. E.; Frail, D. E. J. Biol.

Chem. 1994, 269, 27799. (b) Komano, H.; Seeger, M.; Gandy, S.; Wang, G.

T.; Krafft, G. A.; Fuller, R. S. J. Biol. Chem. 1998, 273, 31648.

(113) (a) Baumeister, R.; Leimer, U.; Zweckbronner, I.; Jakubek, C.; Grunberg, J.;

Haass, C. Genes and function 1997, 1, 149. (b) Levitan, D.; Doyle, T. G.; 
Brousseau, D.; Lee, M. K.; Thinakaran, G.; Slunt, H. H.; Sisodia, S. S.;

Greenwald, I. Proc. Natl. Acad. Sci. U. S. A. 1996, 93, 14940.

(114) Cabaleiro-Lago, C.; Quinlan-Pluck, F.; Lynch, I.; Lindman, S.; Minogue, A. M.; Thulin, E.; Walsh, D. M.; Dawson, K. A.; Linse, S. J. Am. Chem. Soc. 2008, $130,15437$.

(115) Halim, A.; Brinkmalm, G.; Ruetschi, U.; Westman-Brinkmalm, A.; Portelius, E.; Zetterberg, H.; Blennow, K.; Larson, G.; Nilsson, J. Proc. Natl. Acad. Sci. U. S. A. 2011, 108, 11848.

(116) Sinha, S.; Anderson, J. P.; Barbour, R.; Basi, G. S.; Caccavello, R.; Davis, D.; Doan, M.; Dovey, H. F.; Frigon, N.; Hong, J.; Jacobson-Croak, K.; Jewett, N.; Keim, P.; Knops, J.; Lieberburg, I.; Power, M.; Tan, H.; Tatsuno, G.; Tung, J.; Schenk, D.; Seubert, P.; Suomensaari, S. M.; Wang, S. W.; Walker, D.; Zhao, J.; McConlogue, L.; John, V. Nature 1999, 402, 537.

(117) (a) Francis, R.; McGrath, G.; Zhang, J. H.; Ruddy, D. A.; Sym, M.; Apfeld, J.; Nicoll, M.; Maxwell, M.; Hai, B.; Ellis, M. C.; Parks, A. L.; Xu, W.; Li, J. H.; Gurney, M.; Myers, R. L.; Himes, C. S.; Hiebsch, R.; Ruble, C.; Nye, J. S.; Curtis, D. Dev. Cell 2002, 3, 85. (b) De Strooper, B. Neuron 2003, 38, 9. (c) St George-Hyslop, P.; Schmitt-Ulms, G. Nature 2010, 467, 36.

(118) Tabaton, M.; Zhu, X. W.; Perry, G.; Smith, M. A.; Giliberto, L. Exp. Neurol. 2010, 221,18 .

(119) Guglielmotto, M.; Aragno, M.; Autelli, R.; Giliberto, L.; Novo, E.; Colombatto, S.; Danni, O.; Parola, M.; Smith, M. A.; Perry, G.; Tamagno, E.; Tabaton, M. J. Neurochem. 2009, 108, 1045.

(120) (a) Allinson, T. M. J.; Parkin, E. T.; Turner, A. J.; Hooper, N. M. J. Neurosci. Res. 2003, 74, 342. (b) Portelius, E.; Price, E.; Brinkmalm, G.; Stiteler, M.; 
Olsson, M.; Persson, R.; Westman-Brinkmalm, A.; Zetterberg, H.; Simon, A. J.; Blennow, K. Neurobiology of Aging 2011, 32, 1090.

(121) Rogaeva, E.; Meng, Y.; Lee, J. H.; Gu, Y.; Kawarai, T.; Zou, F.; Katayama, T.; Baldwin, C. T.; Cheng, R.; Hasegawa, H.; Chen, F.; Shibata, N.; Lunetta, K. L.; Pardossi-Piquard, R.; Bohm, C.; Wakutani, Y.; Cupples, L. A.; Cuenco, K. T.; Green, R. C.; Pinessi, L.; Rainero, I.; Sorbi, S.; Bruni, A.; Duara, R.; Friedland, R. P.; Inzelberg, R.; Hampe, W.; Bujo, H.; Song, Y. Q.; Andersen, O. M.; Willnow, T. E.; Graff-Radford, N.; Petersen, R. C.; Dickson, D.; Der, S. D.; Fraser, P. E.; Schmitt-Ulms, G.; Younkin, S.; Mayeux, R.; Farrer, L. A.; St George-Hyslop, P. Nature Genetics 2007, 39, 168.

(122) Jaworski, T.; Dewachter, I.; Lechat, B.; Gees, M.; Kremer, A.; Demedts, D.; Borghgraef, P.; Devijver, H.; Kugler, S.; Patel, S.; Woodgett, J. R.; Van Leuven, F. Nature 2011, 480, E4.

(123) Nikolaev, A.; McLaughlin, T.; O'Leary, D. D. M.; Tessier-Lavigne, M. Nature 2009, 457, 981.

(124) Hendriks, L.; Vanduijn, C. M.; Cras, P.; Cruts, M.; Vanhul, W.; Vanharskamp, F.; Warren, A.; McInnis, M. G.; Antonarakis, S. E.; Martin, J. J.; Hofman, A.; Vanbroeckhoven, C. Nature Genetics 1992, 1, 218.

(125) Farzan, M.; Schnitzler, C. E.; Vasilieva, N.; Leung, D.; Choe, H. Proc. Natl. Acad. Sci. U. S. A. 2000, 97, 9712.

(126) Selkoe, D. J.; Podlisny, M. B.; Joachim, C. L.; Vickers, E. A.; Lee, G.; Fritz, L. C.; Oltersdorf, T. Proc. Natl. Acad. Sci. U. S. A. 1988, 85, 7341.

(127) Sinha, S.; Dovey, H. F.; Seubert, P.; Ward, P. J.; Blacher, R. W.; Blaber, M.; Bradshaw, R. A.; Arici, M.; Mobley, W. C.; Lieberburg, I. J. Biol. Chem. 1990, 265, 8983. 
(128) Goate, A. M. Cell. Mol. Life Sci. 1998, 54, 897.

(129) Mullan, M.; Crawford, F.; Axelman, K.; Houlden, H.; Lilius, L.; Winblad, B.; Lannfelt, L. Nature Genetics 1992, 1, 345.

(130) (a) Chartierharlin, M. C.; Crawford, F.; Houlden, H.; Warren, A.; Hughes, D.; Fidani, L.; Goate, A.; Rossor, M.; Roques, P.; Hardy, J.; Mullan, M. Nature 1991, 353, 844. (b) Sinha, S.; Lieberburg, I. Proc. Natl. Acad. Sci. U. S. A. 1999, 96, 11049.

(131) Nilsberth, C.; Westlind-Danielsson, A.; Eckman, C. B.; Condron, M. M.; Axelman, K.; Forsell, C.; Stenh, C.; Luthman, J.; Teplow, D. B.; Younkin, S. G.; Naslund, J.; Lannfelt, L. Nature Neuroscience 2001, 4, 887.

(132) (a) Grabowski, T. J.; Cho, H. S.; Vonsattel, J. P. G.; Rebeck, G. W.; Greenberg, S. M. Ann. Neurol. 2001, 49, 697. (b) Van Nostrand, W. E.; Melchor, J. P.; Cho, H. S.; Greenberg, S. M.; Rebeck, G. W. J. Biol. Chem. 2001, 276, 32860. (133) Mucke, L.; Masliah, E.; Yu, G. Q.; Mallory, M.; Rockenstein, E. M.; Tatsuno, G.; Hu, K.; Kholodenko, D.; Johnson-Wood, K.; McConlogue, L. J. Neurosci. 2000, 20, 4050 .

(134) Takahashi, T.; Mihara, H. Chem. Comm. 2012, 48, 1568.

(135) Sian, A. K.; Frears, E. R.; El-Agnaf, O. M. A.; Patel, B. P.; Manca, M. F.; Siligardi, G.; Hussain, R.; Austen, B. M. Biochem. J. 2000, 349, 299.

(136) Schierle, G. S. K.; van de Linde, S.; Erdelyi, M.; Esbjorner, E. K.; Klein, T.; Rees, E.; Bertoncini, C. W.; Dobson, C. M.; Sauer, M.; Kaminski, C. F. J. Am. Chem. Soc. 2011, 133, 12902.

(137) Zhang, Y.; McLaughlin, R.; Goodyer, C.; LeBlanc, A. J. Cell Biol. 2002, 156, 519.

(138) Nagy, Z.; Esiri, M. M. Neurobiology of Aging 1997, 18, 565. 
(139) (a) Nagy, Z.; Esiri, M. M.; Cato, A. M.; Smith, A. D. Acta Neuropathol. 1997, 94, 6. (b) Nagy, Z.; Esiri, M. M.; Smith, A. D. Neuroscience 1998, 87, 731.

(140) (a) Chen, Q. S.; Kagan, B. L.; Hirakura, Y.; Xie, C. W. J. Neurosci. Res. 2000, 60, 65. (b) Miñano-Molina, A. J.; España, J.; Martin, E.; Barneda-Zahonero, B.; Fadó, R.; Solé, M.; Trullás, R.; Saura, C. A.; Rodríguez-Alvarez, J. J. Biol. Chem. 2011, 286, 27311.

(141) (a) Chapman, P. F.; White, G. L.; Jones, M. W.; Cooper-Blacketer, D.; Marshall, V. J.; Irizarry, M.; Younkin, L.; Good, M. A.; Bliss, T. V. P.; Hyman, B. T.; Younkin, S. G.; Hsiao, K. K. Nature Neuroscience 1999, 2 , 271. (b) Larson, J.; Lynch, G.; Games, D.; Seubert, P. Brain Res. 1999, 840, 23. (c) Giacchino, J.; Criado, J. R.; Games, D.; Henriksen, S. Brain Res. 2000, 876, 185. (d) Lanz, T. A.; Carter, D. B.; Merchant, K. M. Neurobiol. Dis. 2003, 13, 246. (e) Spires, T. L.; Meyer-Luehmann, M.; Stern, E. A.; McLean, P. J.; Skoch, J.; Nguyen, P. T.; Bacskai, B. J.; Hyman, B. T. J. Neurosci. 2005, 25, 7278. (f) Almeida, C. G.; Tampellini, D.; Takahashi, R. H.; Greengard, P.; Lin, M. T.; Snyder, E. M.; Gouras, G. K. Neurobiol. Dis. 2005, 20, 187.

(142) Snyder, E. M.; Nong, Y.; Almeida, C. G.; Paul, S.; Moran, T.; Choi, E. Y.; Nairn, A. C.; Salter, M. W.; Lombroso, P. J.; Gouras, G. K.; Greengard, P. Nature Neuroscience 2005, 8, 1051.

(143) Kim, J. H.; Anwyl, R.; Suh, Y. H.; Djamgoz, M. B. A.; Rowan, M. J. J. Neurosci. 2001, 21, 1327.

(144) Kamenetz, F.; Tomita, T.; Hsieh, H.; Seabrook, G.; Borchelt, D.; Iwatsubo, T.; Sisodia, S.; Malinow, R. Neuron 2003, 37, 925.

(145) (a) Hartley, D. M.; Walsh, D. M.; Ye, C. P. P.; Diehl, T.; Vasquez, S.; Vassilev, P. M.; Teplow, D. B.; Selkoe, D. J. J. Neurosci. 1999, 19, 8876. (b) Moechars, 
D.; Dewachter, I.; Lorent, K.; Reverse, D.; Baekelandt, V.; Naidu, A.;

Tesseur, I.; Spittaels, K.; Van Den Haute, C.; Checler, F.; Godaux, E.; Cordell, B.; Van Leuven, F. J. Biol. Chem. 1999, 274, 6483. (c) Billings, L. M.; Oddo, S.; Green, K. N.; McGaugh, J. L.; LaFerla, F. M. Neuron 2005, 45, 675.

(146) (a) Walsh, D. M.; Klyubin, I.; Fadeeva, J. V.; Cullen, W. K.; Anwyl, R.; Wolfe, M. S.; Rowan, M. J.; Selkoe, D. J. Nature 2002, 416, 535. (b) Wang, H. W.; Pasternak, J. F.; Kuo, H.; Ristic, H.; Lambert, M. P.; Chromy, B.; Viola, K. L.; Klein, W. L.; Stine, W. B.; Krafft, G. A.; Trommer, B. L. Brain Res. 2002, 924, 133. (c) Townsend, M.; Shankar, G. M.; Mehta, T.; Walsh, D. M.; Selkoe, D. J. J. Physiol.-London 2006, 572, 477. (d) Selkoe, D. J. Behavioral Brain Research 2008, 192, 106. (e) Kessels, H. W.; Nguyen, L. N.; Nabavi, S.; Malinow, R. Nature 2010, 466, E3. (f) Balducci, C.; Beeg, M.; Stravalaci, M.; Bastone, A.; Sclip, A.; Biasini, E.; Tapella, L.; Colombo, L.; Manzoni, C.; Borsello, T.; Chiesa, R.; Gobbi, M.; Salmona, M.; Forloni, G. Proc. Natl. Acad. Sci. U. S. A. 2010, 107, 2295.

(147) (a) Hsieh, H.; Boehm, J.; Sato, C.; Iwatsubo, T.; Tomita, T.; Sisodia, S.; Malinow, R. Neuron 2006, 52, 831. (b) Li, S. M.; Hong, S. Y.; Shepardson, N. E.; Walsh, D. M.; Shankar, G. M.; Selkoe, D. Neuron 2009, 62, 788.

(148) Cullen, W. K.; Suh, Y. H.; Anwyl, R.; Rowan, M. J. Neuroreport 1997, 8, 3213.

(149) Freir, D. B.; Holscher, C.; Herron, C. E. J. Neurophysiol. 2001, 85, 708.

(150) Wang, Q. W.; Walsh, D. M.; Rowan, M. J.; Selkoe, D. J.; Anwyl, R. J. Neurosci. 2004, 24, 3370.

(151) Bell, K. F. S.; Ducatenzeiler, A.; Ribeiro-da-Silva, A.; Duff, K.; Bennett, D. A.; Cuello, A. C. Neurobiology of Aging 2006, 27, 1644.

(152) Lee, B. Y.; Ban, J. Y.; Seong, Y. H. Neurosci. Res. 2005, 52, 347. 
(153) (a) Wang, H. Y.; Lee, D. H. S.; D'Andrea, M. R.; Peterson, P. A.; Shank, R. P.; Reitz, A. B. J. Biol. Chem. 2000, 275, 5626. (b) Wang, H. Y.; Lee, D. H. S.; Davis, C. B.; Shank, R. P. J. Neurochem. 2000, 75, 1155.

(154) (a) Kihara, T.; Shimohama, S.; Sawada, H.; Kimura, J.; Kume, T.; Kochiyama, H.; Maeda, T.; Akaike, A. Ann. Neurol. 1997, 42, 159. (b) Liu, Q. S.; Kawai, H.; Berg, D. K. Proc. Natl. Acad. Sci. U. S. A. 2001, 98, 4734.

(155) (a) Dineley, K. T.; Bell, K. A.; Bui, D.; Sweatt, J. D. J. Biol. Chem. 2002, 277, 25056. (b) Spencer, J. P.; Weil, A.; Hill, K.; Hussain, I.; Richardson, J. C.; Cusdin, F. S.; Chen, Y. H.; Randall, A. D. Neuroscience 2006, 137, 795. (c) Abbott, J. J.; Howlett, D. R.; Francis, P. T.; Williams, R. J. Neurobiology of Aging 2008, 29, 992.

(156) Kihara, T.; Shimohama, S.; Urushitani, M.; Sawada, H.; Kimura, J.; Kume, T.; Maeda, T.; Akaike, A. Brain Res. 1998, 792, 331.

(157) Maelicke, A. Dement. Geriatr. Cogn. Disord. 2000, 11, 11.

(158) Pettit, D. L.; Shao, Z.; Yakel, J. L. J. Neurosci. 2001, 21.

(159) Louzada, P. R.; Lima, A. C. P.; Mendonca-Silva, D. L.; Noel, F.; De Mello, F. G.; Ferreira, S. T. FASEB J. 2004, 18, 511.

(160) (a) Geula, C.; Wu, C. K.; Saroff, D.; Lorenzo, A.; Yuan, M. L.; Yankner, B. A. Nature Medicine 1998, 4, 827. (b) Combs, C. K.; Karlo, J. C.; Kao, S. C.; Landreth, G. E. J. Neurosci. 2001, 21, 1179. (c) Qin, S.; Colin, C.; Hinners, I.; Gervais, A.; Cheret, C.; Mallat, M. J. Neurosci. 2006, 26, 3345. (d) MeyerLuehmann, M.; Spires-Jones, T. L.; Prada, C.; Garcia-Alloza, M.; de Calignon, A.; Rozkalne, A.; Koenigsknecht-Talboo, J.; Holtzman, D. M.; Bacskai, B. J.; Hyman, B. T. Nature 2008, 451, 720. 
(161) Wyss-Coray, T.; Masliah, E.; Mallory, M.; McConlogue, L.; Johnson-Wood, K.; Lin, C.; Mucke, L. Nature 1997, 389, 603.

(162) Masliah, E. Nature 2008, 451, 638.

(163) Nagele, R. G.; Wegiel, J.; Venkataraman, V.; Imaki, H.; Wang, K. C.; Wegiel, J. Neurobiology of Aging 2004, 25, 663.

(164) Simard, A. R.; Soulet, D.; Gowing, G.; Julien, J. P.; Rivest, S. Neuron 2006, 49, 489.

(165) Westin, K.; Buchhave, P.; Nielsen, H. M.; Minthon, L.; Janciauskiene, S. M.; Hansson, O. Plos One 2012, 7, e30525.

(166) Ramirez, B. G.; Blazquez, C.; del Pulgar, T. G.; Guzman, N.; de Ceballos, M. A. L. J. Neurosci. 2005, 25, 1904.

(167) Selkoe, D. J. Neuron 2001, 32, 177.

(168) Llovera, R. E.; de Tullio, M.; Alonso, L. G.; Leissring, M. A.; Kaufman, S. B.; Roher, A. E.; Gay, G. D.; Morelli, L.; Castano, E. M. J. Biol. Chem. 2008, 283,17039 .

(169) Kumar, S.; Singh, S.; Hinze, D.; Josten, M.; Sahl, H. G.; Siepmann, M.; Walter, J. J. Biol. Chem. 2012, 287, 8641 .

(170) Hellstrom-Lindahl, E.; Ravid, R.; Nordberg, A. Neurobiology of Aging 2008, $29,210$.

(171) (a) Hu, J.; Miyatake, F.; Aizu, Y.; Nakagawa, H.; Nakamura, S.; Tamaoka, A.; Takahash, R.; Urakami, K.; Shoji, M. Neurosci. Lett. 1999, 277, 65. (b) Hu, J. G.; Igarashi, A.; Kamata, M.; Nakagawa, H. J. Biol. Chem. 2001, 276, 47863.

(172) (a) Williams, R. Nature 2011, 475, S5. (b) Buckholtz, N. S. Nature 2011, 475, S8. 
(173) Jack, C. R.; Knopman, D. S.; Jagust, W. J.; Shaw, L. M.; Aisen, P. S.; Weiner, M. W.; Petersen, R. C.; Trojanowski, J. Q. Lancet Neurol. 2010, 9, 119.

(174) (a) Hampel, H.; Frank, R.; Broich, K.; Teipel, S. J.; Katz, R. G.; Hardy, J.; Herholz, K.; Bokde, A. L. W.; Jessen, F.; Hoessler, Y. C.; Sanhai, W. R.; Zetterberg, H.; Woodcock, J.; Blennow, K. Nature Rev. Drug Disc. 2010, 9 , 560. (b) Humpel, C. Trends in Biotechnology 2011, 29, 26.

(175) Blennow, K.; Hampel, H. Lancet Neurol. 2003, 2, 605.

(176) Skoog, I.; Davidsson, P.; Aevarsson, O.; Vanderstichele, H.; Vanmechelen, E.; Blennow, K. Dement. Geriatr. Cogn. Disord. 2003, 15, 169.

(177) Gustafson, D. R.; Skoog, I.; Rosengren, L.; Zetterberg, H.; Blennow, K. J. Neurol. Neurosurg. Psychiatry 2007, 78, 461.

(178) Stomrud, E.; Hansson, O.; Blennow, K.; Minthon, L.; Londos, E. Dement. Geriatr. Cogn. Disord. 2007, 24, 118.

(179) Mattsson, N.; Zetterberg, H.; Hansson, O.; Andreasen, N.; Parnetti, L.; Jonsson, M.; Herukka, S. K.; van der Flier, W. M.; Blankenstein, M. A.; Ewers, M.; Rich, K.; Kaiser, E.; Verbeek, M.; Tsolaki, M.; Mulugeta, E.; Rosen, E.; Aarsland, D.; Visser, P. J.; Schroder, J.; Marcusson, J.; de Leon, M.; Hampel, H.; Scheltens, P.; Pirttila, T.; Wallin, A.; Jonhagen, M. E.; Minthon, L.; Winblad, B.; Blennow, K. J. Am. Med. Assoc. 2009, 302, 385.

(180) Ray, S.; Britschgi, M.; Herbert, C.; Takeda-Uchimura, Y.; Boxer, A.; Blennow, K.; Friedman, L. F.; Galasko, D. R.; Jutel, M.; Karydas, A.; Kaye, J. A.; Leszek, J.; Miller, B. L.; Minthon, L.; Quinn, J. F.; Rabinovici, G. D.; Robinson, W. H.; Sabbagh, M. N.; So, Y. T.; Sparks, D. L.; Tabaton, M.; Tinklenberg, J.; Yesavage, J. A.; Tibshirani, R.; Wyss-Coray, T. Nature Medicine 2007, 13, 1359. 
(181) (a) Okereke, O. I.; Xia, W. M.; Selkoe, D. J.; Grodstein, F. Arch. Neurol. 2009, 66, 1247. (b) Yaffe, K.; Weston, A.; Graff-Radford, N. R.; Satterfield, S.; Simonsick, E. M.; Younkin, S. G.; Younkin, L. H.; Kuller, L.; Ayonayon, H. N.; Ding, J. Z.; Harris, T. B. J. Am. Med. Assoc. 2011, 305, 261. (c) Yaffe, K.; Weston, A. J. Am. Med. Assoc. 2011, 305, 1655.

(182) Reddy, M. M.; Wilson, R.; Wilson, J.; Connell, S.; Gocke, A.; Hynan, L.; German, D.; Kodadek, T. Cell 2011, 144, 132.

(183) (a) Mathis, C. A.; Wang, Y. M.; Holt, D. P.; Huang, G. F.; Debnath, M. L.; Klunk, W. E. J. Med. Chem. 2003, 46, 2740. (b) Klunk, W. E.; Engler, H.; Nordberg, A.; Wang, Y. M.; Blomqvist, G.; Holt, D. P.; Bergstrom, M.; Savitcheva, I.; Huang, G. F.; Estrada, S.; Ausen, B.; Debnath, M. L.; Barletta, J.; Price, J. C.; Sandell, J.; Lopresti, B. J.; Wall, A.; Koivisto, P.; Antoni, G.; Mathis, C. A.; Langstrom, B. Ann. Neurol. 2004, 55, 306. (c) Fagan, A. M.; Mintun, M. A.; Mach, R. H.; Lee, S. Y.; Dence, C. S.; Shah, A. R.; LaRossa, G. N.; Spinner, M. L.; Klunk, W. E.; Mathis, C. A.; DeKosky, S. T.; Morris, J. C.; Holtzman, D. M. Ann. Neurol. 2006, 59, 512. (d) Forsberg, A.; Engler, H.; Almkvist, O.; Blomquist, G.; Hagman, G.; Wall, A.; Ringheim, A.; Langstrom, B.; Nordberg, A. Neurobiology of Aging 2008, 29, 1456. (e) Tapiola, T.; Alafuzoff, I.; Herukka, S. K.; Parkkinen, L.; Hartikainen, P.; Soininen, H.; Pirttila, T. Arch. Neurol. 2009, 66, 382.

(184) (a) Cordeiro, M. F.; Guo, L.; Coxon, K. M.; Duggan, J.; Nizari, S.; Normando, E. M.; Sensi, S. L.; Sillito, A. M.; Fitzke, F. W.; Salt, T. E.; Moss, S. E. Cell Death Dis. 2010, 1. (b) Guo, L.; Duggan, J.; Cordeiro, M. F. Current Alzheimer Research 2010, 7, 3. 
(185) (a) Nielsen, H. M.; Minthon, L.; Londos, E.; Blennow, K.; Miranda, E.; Perez, J.; Crowther, D. C.; Lomas, D. A.; Janciauskiene, S. M. Neurology 2007, 69, 1569. (b) Schneider, P.; Hampel, H.; Buerger, K. CNS Neurosci. Ther. 2009, 15,358 .

(186) Li, R.; Lindholm, K.; Yang, L. B.; Yue, X.; Citron, M.; Yao, R. Q.; Beach, T.; Sue, L.; Sabbagh, M.; Cai, H. B.; Wong, P.; Price, D.; Shen, Y. Proc. Natl. Acad. Sci. U. S. A. 2004, 101, 3632.

(187) Johnson-Wood, K.; Lee, M.; Motter, R.; Hu, K.; Gordon, G.; Barbour, R.; Khan, K.; Gordon, M.; Tan, H.; Games, D.; Lieberburg, I.; Schenk, D.; Seubert, P.; McConlogue, L. Proc. Natl. Acad. Sci. U. S. A. 1997, 94, 1550. (188) (a) DeMattos, R. B.; Bales, K. R.; Cummins, D. J.; Dodart, J. C.; Paul, S. M.; Holtzman, D. M. Proc. Natl. Acad. Sci. U. S. A. 2001, 98, 8850. (b) Shankar, G. M.; Bloodgood, B. L.; Townsend, M.; Walsh, D. M.; Selkoe, D. J.; Sabatini, B. L. J. Neurosci. 2007, 27, 2866.

(189) (a) Kim, K. S.; Wen, G. Y.; Bancher, C.; Chen, C. M. J.; Sapienza, V. J.; Hong, H.; Wisniewski, H. M. Neurosci. Res. Commun. 1990, 7, 113. (b) Podlisny, M. B.; Ostaszewski, B. L.; Squazzo, S. L.; Koo, E. H.; Rydell, R. E.; Teplow, D. B.; Selkoe, D. J. J. Biol. Chem. 1995, 270, 9564. (c) Wong, H. E.; Qi, W.; Choi, H. M.; Fernandez, E.; Kwon, I. ACS Chem. Neurosci. 2011, 2, 645. (190) (a) Haass, C.; Schlossmacher, M. G.; Hung, A. Y.; Vigopelfrey, C.; Mellon, A.; Ostaszewski, B. L.; Lieberburg, I.; Koo, E. H.; Schenk, D.; Teplow, D. B.; Selkoe, D. J. Nature 1992, 359, 322. (b) Solomon, B.; Koppel, R.; Frankel, D.; HananAharon, E. Proc. Natl. Acad. Sci. U. S. A. 1997, 94, 4109.

(191) Bieschke, J.; Herbst, M.; Wiglenda, T.; Friedrich, R. P.; Boeddrich, A.; Schiele, F.; Kleckers, D.; del Amo, J. M. L.; Gruning, B. A.; Wang, Q. W.; Schmidt, 
M. R.; Lurz, R.; Anwyl, R.; Schnoegl, S.; Fandrich, M.; Frank, R. F.; Reif, B.; Gunther, S.; Walsh, D. M.; Wanker, E. E. Nature Chemical Biology 2012, 8, 93.

(192) Sergeant, N.; Bombois, S.; Ghestem, A.; Drobecq, H.; Kostanjevecki, V.;

Missiaen, C.; Wattez, A.; David, J. P.; Vanmechelen, E.; Sergheraert, C.;

Delacourte, A. J. Neurochem. 2003, 85, 1581.

(193) Barghorn, S.; Nimmrich, V.; Striebinger, A.; Krantz, C.; Keller, P.; Janson, B.; Bahr, M.; Schmidt, M.; Bitner, R. S.; Harlan, J.; Barlow, E.; Ebert, U.; Hillen, H. J. Neurochem. 2005, 95, 834.

(194) (a) Kayed, R.; Head, E.; Thompson, J. L.; McIntire, T. M.; Milton, S. C.; Cotman, C. W.; Glabe, C. G. Science 2003, 300, 486. (b) Kayed, R.; Head, E.; Sarsoza, F.; Saing, T.; Cotman, C. W.; Necula, M.; Margol, L.; Wu, J.; Breydo, L.; Thompson, J. L.; Rasool, S.; Gurlo, T.; Butler, P.; Glabe, C. G. Mol. Neurodegener. 2007, 2. (c) Glabe, C. G. J. Biol. Chem. 2008, 283, 29639.

(195) McLaurin, J.; Cecal, R.; Kierstead, M. E.; Tian, X.; Phinney, A. L.; Manea, M.; French, J. E.; Lambermon, M. H. L.; Darabie, A. A.; Brown, M. E.; Janus, C.; Chishti, M. A.; Horne, P.; Westaway, D.; Fraser, P. E.; Mount, H. T. J.; Przybylski, M.; St George-Hyslop, P. Nature Medicine 2002, 8, 1263. (196) Mamikonyan, G.; Necula, M.; Mkrtichyan, M.; Ghochikyan, A.; Petrushina, I.; Movsesyan, N.; Mina, E.; Kiyatkin, A.; Glabe, C. G.; Cribbs, D. H.; Agadjanyan, M. G. J. Biol. Chem. 2007, 282, 22376.

(197) McLean, D.; Cooke, M. J.; Wang, Y.; Fraser, P.; St George-Hyslop, P.; Shoichet, M. S. J. Controlled Release 2012, in press. 
(198) Lambert, M. P.; Velasco, P. T.; Chang, L.; Viola, K. L.; Fernandez, S.; Lacor, P. N.; Khuon, D.; Gong, Y. S.; Bigio, E. H.; Shaw, P.; De Felice, F. G.; Krafft, G. A.; Klein, W. L. J. Neurochem. 2007, 100, 23.

(199) Jin, M.; Shepardson, N.; Yang, T.; Chen, G.; Walsh, D.; Selkoe, D. J. Proc. Natl. Acad. Sci. U. S. A. 2011, 108, 5819.

(200) Hoyer, W.; Gronwall, C.; Jonsson, A.; Stahl, S.; Hard, T. Proc. Natl. Acad. Sci. U. S. A. 2008, 105, 5099.

(201) Macao, B.; Hoyer, W.; Sandberg, A.; Brorsson, A. C.; Dobson, C. M.; Hard, T. BMC Biotechnol. 2008, 8.

(202) (a) Lambert, M. P.; Barlow, A. K.; Chromy, B. A.; Edwards, C.; Freed, R.; Liosatos, M.; Morgan, T. E.; Rozovsky, I.; Trommer, B.; Viola, K. L.; Wals, P.; Zhang, C.; Finch, C. E.; Krafft, G. A.; Klein, W. L. Proc. Natl. Acad. Sci. U. S. A. 1998, 95, 6448. (b) Andersson, K.; Olofsson, A.; Nielsen, E. H.; Svehag, S. E.; Lundgren, E. Biochem. Biophys. Res. Commun. 2002, 294, 309. (c) Rousseau, F.; Schmykovitz, J.; Serrano, L. Curr. Opin. Struct. Biol. 2006, $16,118$.

(203) Du, Y. S.; Wei, X.; Dodel, R.; Sommer, N.; Hampel, H.; Gao, F.; Ma, Z. Z.; Zhao, L. M.; Oertel, W. H.; Farlow, M. Brain 2003, 126, 1935.

(204) Lambert, M. P.; Viola, K. L.; Chromy, B. A.; Chang, L.; Morgan, T. E.; Yu, J. X.; Venton, D. L.; Krafft, G. A.; Finch, C. E.; Klein, W. L. J. Neurochem. 2001, 79, 595.

(205) Yi, P.; Hadden, C.; Kulanthaivel, P.; Calvert, N.; Annes, W.; Brown, T.; Barbuch, R. J.; Chaudhary, A.; Ayan-Oshodi, M. A.; Ring, B. J. Drug Metab. Dispos. 2010, 38, 554. 
(206) Lacor, P. N.; Buniel, M. C.; Furlow, P. W.; Clemente, A. S.; Velasco, P. T.; Wood, M.; Viola, K. L.; Klein, W. L. J. Neurosci. 2007, 27, 796.

(207) Lesne, S.; Koh, M. T.; Kotilinek, L.; Kayed, R.; Glabe, C. G.; Yang, A.; Gallagher, M.; Ashe, K. H. Nature 2006, 440, 352.

(208) Dobson, C. M. Nature 2003, 426, 884.

(209) Haupt, C.; Leppert, J.; Rönicke, R.; Meinhardt, J.; Yadav, J. K.; Ramachandran, R.; Ohlenschläger, O.; Reymann, K. G.; Görlach, M.; Fändrich, M. Angew. Chem., Int. Ed. Engl. 2012, 51, 1576.

(210) (a) Kuo, Y. M.; Emmerling, M. R.; VigoPelfrey, C.; Kasunic, T. C.; Kirkpatrick, J. B.; Murdoch, G. H.; Ball, M. J.; Roher, A. E. J. Biol. Chem. 1996, 271, 4077. (b) Roher, A. E.; Chaney, M. O.; Kuo, Y. M.; Webster, S. D.; Stine, W. B.; Haverkamp, L. J.; Woods, A. S.; Cotter, R. J.; Tuohy, J. M.; Krafft, G. A.; Bonnell, B. S.; Emmerling, M. R. J. Biol. Chem. 1996, 271, 20631. (c) Enya, M.; Morishima-Kawashima, M.; Yoshimura, M.; Shinkai, Y.; Kusui, K.; Khan, K.; Games, D.; Schenk, D.; Sugihara, S.; Yamaguchi, H.; Ihara, Y. Am. J. Pathol. 1999, 154, 271. (d) Funato, H.; Enya, M.; Yoshimura, M.; Morishima-Kawashima, M.; Ihara, Y. Am. J. Pathol. 1999, 155, 23.

(211) Shekhawat, G. S.; Chand, A.; Sharma, S.; Verawati; Dravid, V. P. Appl. Phys. Lett. 2009, 95, 233114.

(212) Harper, J. D.; Wong, S. S.; Lieber, C. M.; Lansbury, P. T. Chemistry and Biology 1997, 4, 119.

(213) Walsh, D. M.; Klyubin, I.; Fadeeva, J. V.; Rowan, M. J.; Selkoe, D. J. Biochem. Soc. Trans. 2002, 30, 552. 
(214) Chromy, B. A.; Nowak, R. J.; Lambert, M. P.; Viola, K. L.; Chang, L.; Velasco, P. T.; Jones, B. W.; Fernandez, S. J.; Lacor, P. N.; Horowitz, P.; Finch, C. E.; Krafft, G. A.; Klein, W. L. Biochemistry 2003, 42, 12749.

(215) (a) Kayed, R.; Sokolov, Y.; Edmonds, B.; McIntire, T. M.; Milton, S. C.; Hall, J. E.; Glabe, C. G. J. Biol. Chem. 2004, 279, 46363. (b) Lasagna-Reeves, C. A.; Castillo-Carranza, D. L.; Guerrero-Munoz, M. J.; Jackson, G. R.; Kayed, R. Biochemistry 2010, 49, 10039.

(216) Necula, M.; Kayed, R.; Milton, S.; Glabe, C. G. J. Biol. Chem. 2007, 282, 10311.

(217) Bernstein, S. L.; Wyttenbach, T.; Baumketner, A.; Shea, J. E.; Bitan, G.; Teplow, D. B.; Bowers, M. T. J. Am. Chem. Soc. 2005, 127, 2075.

(218) Gellermann, G. P.; Byrnes, H.; Striebinger, A.; Ullrich, K.; Mueller, R.; Hillen, H.; Barghorn, S. Neurobiol. Dis. 2008, 30, 212.

(219) Yu, L. P.; Edalji, R.; Harlan, J. E.; Holzman, T. F.; Lopez, A. P.; Labkovsky, B.; Hillen, H.; Barghorn, S.; Ebert, U.; Richardson, P. L.; Miesbauer, L.; Solomon, L.; Bartley, D.; Walter, K.; Johnson, R. W.; Hajduk, P. J.; Olejniczak, E. T. Biochemistry 2009, 48, 1870.

(220) Bernstein, S. L.; Dupuis, N. F.; Lazo, N. D.; Wyttenbach, T.; Condron, M. M.; Bitan, G.; Teplow, D. B.; Shea, J.-E.; Ruotolo, B. T.; Robinson, C. V.; Bowers, M. T. Nature Chemistry 2009, 1, 326.

(221) (a) Bitan, G.; Kirkitadze, M. D.; Lomakin, A.; Vollers, S. S.; Benedek, G. B.; Teplow, D. B. Proc. Natl. Acad. Sci. U. S. A. 2003, 100, 330. (b) Bitan, G.; Teplow, D. B. Acc. Chem. Res. 2004, 37, 357. 
(222) Narayan, P.; Orte, A.; Clarke, R. W.; Bolognesi, B.; Hook, S.; Ganzinger, K. A.; Meehan, S.; Wilson, M. R.; Dobson, C. M.; Klenerman, D. Nature Structural and Molecular Biology 2012, 19, 79.

(223) Libeu, C. P.; Poksay, K. S.; John, V.; Bredesen, D. E. J. Alzheimers Dis. 2011, $25,547$.

(224) (a) Pollard, H. B.; Arispe, N.; Rojas, E. Cellular and Molecular Neurobiology 1995, 15, 513. (b) Kourie, J. I.; Henry, C. L. Clin. Exp. Pharmacol. Physiol. 2002, 29, 741. (c) Lashuel, H. A.; Hartley, D.; Petre, B. M.; Walz, T.; Lansbury, P. T. Nature 2002, 418, 291. (d) Lashuel, H. A.; Lansbury, P. T. $Q$. Rev. Biophys. 2006, 39, 167. (e) Marx, J. Science 2007, 318, 384. (f) Bischofberger, M.; Gonzalez, M. R.; van der Goot, F. G. Curr. Opin. Cell Biol. 2009, 21, 589. (g) Butterfield, S. M.; Lashuel, H. A. Angew. Chem. 2010, $49,5628$.

(225) (a) Arispe, N.; Rojas, E.; Pollard, H. B. Proc. Natl. Acad. Sci. U. S. A. 1993, 90, 567. (b) Arispe, N.; Pollard, H. B.; Rojas, E. Proc. Natl. Acad. Sci. U. S. A. 1993, 90, 10573. (c) Arispe, N.; Pollard, H. B.; Rojas, E. Ann. N. Y. Acad. Sci. 1994, 747, 256. (d) Arispe, N.; Pollard, H. B.; Rojas, E. Proc. Natl. Acad. Sci. U. S. A. 1996, 93, 1710.

(226) (a) Lin, H.; Bhatia, R.; Lal, R. FASEB J. 2001, 15, 2433. (b) Quist, A.; Doudevski, I.; Lin, H.; Azimova, R.; Ng, D.; Frangione, B.; Kagan, B.; Ghiso, J.; Lal, R. Proc. Natl. Acad. Sci. U. S. A. 2005, 102, 10427.

(227) Hirakura, Y.; Lin, M. C.; Kagan, B. L. J. Neurosci. Res. 1999, 57, 458.

(228) Inoue, S. Amyloid-Journal of Protein Folding Disorders 2008, 15, 223.

(229) Bhatia, R.; Lin, H.; Lal, R. FASEB J. 2000, 14, 1233. 
(230) Parodi, J.; Sepulveda, F. J.; Roa, J.; Opazo, C.; Inestrosa, N. C.; Aguayo, L. G. J. Biol. Chem. 2010, 285, 2506.

(231) Busche, M. A.; Eichhoff, G.; Adelsberger, H.; Abramowski, D.; Wiederhold, K. H.; Haass, C.; Staufenbiel, M.; Konnerth, A.; Garaschuk, O. Science 2008, 321,1686

(232) Green, K. N.; Demuro, A.; Akbari, Y.; Hitt, B. D.; Smith, I. F.; Parker, I.; LaFerla, F. M. J. Cell Biol. 2008, 181, 1107.

(233) Clinton, L. K.; Blurton-Jones, M.; Myczek, K.; Trojanowski, J. Q.; LaFerla, F. M. J. Neurosci. 2010, 30, 7281.

(234) Fagan, A. M.; Mintun, M. A.; Shah, A. R.; Aldea, P.; Roe, C. M.; Mach, R. H.; Marcus, D.; Morris, J. C.; Holtzman, D. M. EMBO Mol. Med. 2009, 1, 371.

(235) Aderinwale, O. G.; Ernst, H. W.; Mousa, S. A. Am. J. Alzheimers Dis. Other Dement. 2010, 25, 414.

(236) Miller, G. Science 2010, 329, 502.

(237) Howard, R.; McShane, R.; Lindesay, J.; Ritchie, C.; Baldwin, A.; Barber, R.; Burns, A.; Dening, T.; Findlay, D.; Holmes, C.; Hughes, A.; Jacoby, R.; Jones, R.; Jones, R.; McKeith, I.; Macharouthu, A.; O'Brien, J.; Passmore, P.; Sheehan, B.; Juszczak, E.; Katona, C.; Hills, R.; Knapp, M.; Ballard, C.; Brown, R.; Banerjee, S.; Onions, C.; Griffin, M.; Adams, J.; Gray, R.; Johnson, T.; Bentham, P.; Phillips, P. New England Journal of Medicine 2012, 366, 893.

(238) Matharu, B.; Gibson, G.; Parsons, R.; Huckerby, T. N.; Moore, S. A.; Cooper, L. J.; Millichamp, R.; Allsop, D.; Austen, B. J. Neurol. Sci. 2009, 280, 49. (239) Coyle, J.; Kershaw, P. Biol. Psychiatry 2001, 49, 289.

(240) Lipton, S. A. Nature Rev. Drug Disc. 2006, 5, 160. 
(241) De Felice, F. G.; Velasco, P. T.; Lambert, M. P.; Viola, K.; Fernandez, S. J.; Ferreira, S. T.; Klein, W. L. J. Biol. Chem. 2007, 282, 11590.

(242) (a) Hamaguchi, T.; Ono, K.; Yamada, M. Cell. Mol. Life Sci. 2006, 63, 1538.

(b) Doig, A. J. Curr. Opin. Drug Discov. Devel. 2007, 10, 533. (c) Amijee, H.; Madine, J.; Middleton, D. A.; Doig, A. J. Biochem. Soc. Trans. 2009, 37, 692. (d) Hawkes, C. A.; Ng, V.; McLaurin, J. Drug Dev. Res. 2009, 70, 111.

(243) (a) Gervais, F.; Paquette, J.; Morissette, C.; Krzywkowski, P.; Yu, M.; Azzi, M.; Lacombe, D.; Kong, X. Q.; Aman, A.; Laurin, J.; Szarek, W. A.; Tremblay, P. Neurobiology of Aging 2007, 28, 537. (b) Aisen, P. S.; Gauthier, S.; Vellas, B.; Briand, R.; Saurnier, D.; Laurin, J.; Garceau, D. Current Alzheimer Research 2007, 4, 473.

(244) Aisen, P. S.; Saumier, D.; Briand, R.; Laurin, J.; Gervais, F.; Tremblay, P.; Garceau, D. Neurology 2006, 67, 1757.

(245) (a) Fraser, P. E.; Nguyen, J. T.; Chin, D. T.; Kirschner, D. A. J. Neurochem. 1992, 59, 1531. (b) Lorenzo, A.; Yankner, B. A. Proc. Natl. Acad. Sci. U. S. A. 1994, 91, 12243. (c) Carter, D. B.; Chou, K. C. Neurobiology of Aging 1998, 19, 37. (d) Frid, P.; Anisimov, S. V.; Popovic, N. Brain Res. Rev. 2007, 53, 135. (e) Pedersen, M. O.; Mikkelsen, K.; Behrens, M. A.; Pedersen, J. S.; Enghild, J. J.; Skrydstrup, T.; Malmendal, A.; Nielsen, N. C. J. Phys. Chem. B 2010, 114, 16003. (f) Bose, P. P.; Chatterjee, U.; Xie, L.; Johansson, J.; Gothelid, E.; Arvidsson, P. I. ACS Chem. Neurosci. 2010, 1, 315.

(246) Buell, A. K.; Dobson, C. M.; Knowles, T. P. J.; Welland, M. E. Biophys. J. 2010, 99, 3492.

(247) Necula, M.; Breydo, L.; Milton, S.; Kayed, R.; van der Veer, W. E.; Tone, P.; Glabe, C. G. Biochemistry 2007, 46, 8850. 
(248) (a) Gorman, P. M.; Yip, C. M.; Fraser, P. E.; Chakrabartty, A. J. Mol. Biol. 2003, 325, 743. (b) Lee, J.; Culyba, E. K.; Powers, E. T.; Kelly, J. W. Nature Chemical Biology 2011, 7, 602.

(249) Ladiwala, A. R. A.; Dordick, J. S.; Tessier, P. M. J. Biol. Chem. 2011, 286, 3209.

(250) Ramassamy, C. Eur. J. Pharmacol. 2006, 545, 51.

(251) Kelsey, N. A.; Wilkins, H. M.; Linseman, D. A. Molecules 2010, 15, 7792.

(252) (a) Ehrnhoefer, D. E.; Bieschke, J.; Boeddrich, A.; Herbst, M.; Masino, L.; Lurz, R.; Engemann, S.; Pastore, A.; Wanker, E. E. Nat. Struct. Mol. Biol. 2008, 15, 558. (b) Bieschke, J.; Russ, J.; Friedrich, R. P.; Ehrnhoefer, D. E.; Wobst, H.; Neugebauer, K.; Wanker, E. E. Proc. Natl. Acad. Sci. U. S. A. 2010, 107, 7710.

(253) Ono, K.; Yoshiike, Y.; Takashima, A.; Hasegawa, K.; Naiki, H.; Yamada, M. J. Neurochem. 2003, 87, 172.

(254) Jones, O. G.; Mezzenga, R. Soft Matter 2012, 8, 876.

(255) Hamaguchi, T.; Ono, K.; Murase, A.; Yamada, M. Am. J. Pathol. 2009, 175, 2557.

(256) Yang, F. S.; Lim, G. P.; Begum, A. N.; Ubeda, O. J.; Simmons, M. R.; Ambegaokar, S. S.; Chen, P. P.; Kayed, R.; Glabe, C. G.; Frautschy, S. A.; Cole, G. M. J. Biol. Chem. 2005, 280, 5892.

(257) (a) Feng, Y.; Wang, X. P.; Yang, S. G.; Wang, Y. J.; Zhang, X.; Du, X. T.; Sun, X. X.; Zhao, M.; Huang, L.; Liu, R. T. Neurotoxicology 2009, 30, 986. (b) Ladiwala, A. R. A.; Lin, J. C.; Bale, S. S.; Marcelino-Cruz, A. M.; Bhattacharya, M.; Dordick, J. S.; Tessier, P. M. J. Biol. Chem. 2010, 285, 24228. 
(258) Frydman-Marom, A.; Rechter, M.; Shefler, I.; Bram, Y.; Shalev, D. E.; Gazit, E. Angew. Chem. Int. Ed. Engl. 2009, 48, 1981.

(259) (a) Cherny, R. A.; Legg, J. T.; McLean, C. A.; Fairlie, D. P.; Huang, X. D.; Atwood, C. S.; Beyreuther, K.; Tanzi, R. E.; Masters, C. L.; Bush, A. I. J. Biol. Chem. 1999, 274, 23223. (b) Raman, B.; Ban, T.; Yamaguchi, K.; Sakai, M.; Kawai, T.; Naiki, H.; Goto, Y. J. Biol. Chem. 2005, 280, 16157.

(260) Cherny, R. A.; Atwood, C. S.; Xilinas, M. E.; Gray, D. N.; Jones, W. D.; McLean, C. A.; Barnham, K. J.; Volitakis, I.; Fraser, F. W.; Kim, Y. S.; Huang, X. D.; Goldstein, L. E.; Moir, R. D.; Lim, J. T.; Beyreuther, K.; Zheng, H.; Tanzi, R. E.; Masters, C. L.; Bush, A. I. Neuron 2001, 30, 665. (261) (a) Lannfelt, L.; Blennow, K.; Zetterberg, H.; Batsman, S.; Ames, D.; Hrrison, J.; Masters, C. L.; Targum, S.; Bush, A. I.; Murdoch, R.; Wilson, J.; Ritchie, C. W. Lancet Neurol. 2008, 7, 779. (b) Adlard, P. A.; Cherny, R. A.; Finkelstein, D. I.; Gautier, E.; Robb, E.; Cortes, M.; Volitakis, I.; Liu, X.; Smith, J. P.; Perez, K.; Laughton, K.; Li, Q. X.; Charman, S. A.; Nicolazzo, J. A.; Wilkins, S.; Deleva, K.; Lynch, T.; Kok, G.; Ritchie, C. W.; Tanzi, R. E.; Cappai, R.; Masters, C. L.; Barnham, K. J.; Bush, A. I. Neuron 2008, 59, 43. (c) Faux, N. G.; Ritchie, C. W.; Gunn, A.; Rembach, A.; Tsatsanis, A.; Bedo, J.; Harrison, J.; Lannfelt, L.; Blennow, K.; Zetterberg, H.; Ingelsson, M.; Masters, C. L.; Tanzi, R. E.; Cummings, J. L.; Herd, C. M.; Bush, A. I. J. Alzheimers Dis. 2010, 20, 509.

(262) Choi, J. S.; Braymer, J. J.; Nanga, R. P. R.; Ramamoorthy, A.; Lim, M. H. Proc. Natl. Acad. Sci. U. S. A. 2010, 107, 21990.

(263) Scott, L. E.; Telpoukhovskaia, M.; Rodriguez-Rodriguez, C.; Merkel, M.; Bowen, M. L.; Page, B. D. G.; Green, D. E.; Storr, T.; Thomas, F.; Allen, D. 
D.; Lockman, P. R.; Patrick, B. O.; Adam, M. J.; Orvig, C. Chem. Sci. 2011, 2, 642.

(264) Folk, D. S.; Franz, K. J. J. Am. Chem. Soc. 2010, 132, 4994.

(265) Schugar, H.; Green, D. E.; Bowen, M. L.; Scott, L. E.; Storr, T.; Bohmerle, K.;

Thomas, F.; Allen, D. D.; Lockman, P. R.; Merkel, M.; Thompson, K. H.;

Orvig, C. Angew. Chem. Int. Ed. Engl. 2007, 46, 1716.

(266) (a) Arispe, N.; Pollard, H. B.; Rojas, E. Mol. Cell. Biochem. 1994, 140, 119. (b) Simakova, O.; Arispe, N. J. Biochemistry 2006, 45, 5907.

(267) (a) Snow, A. D.; Mar, H.; Nochlin, D.; Kimata, K.; Kato, M.; Suzuki, S.;

Hassell, J.; Wight, T. N. Am. J. Pathol. 1988, 133, 456. (b) McLaurin, J.;

Franklin, T.; Zhang, X. Q.; Deng, J. P.; Fraser, P. E. Eur. J. Biochem. 1999, 266, 1101. (c) McLaurin, J.; Yang, D. S.; Yip, C. M.; Fraser, P. E. Journal of Structural Biology 2000, 130, 259.

(268) Bravo, R.; Arimon, M.; Valle-Delgado, J. J.; Garcia, R.; Durany, N.; Castel, S.;

Cruz, M.; Ventura, S.; Fernandez-Busquets, X. J. Biol. Chem. 2008, 283, 32471.

(269) Leveugle, B.; Scanameo, A.; Ding, W.; Fillit, H. Neuroreport 1994, 5, 1389.

(270) Miura, Y.; Yasuda, K.; Yamamoto, K.; Koike, M.; Nishida, Y.; Kobayashi, K. Biomacromolecules 2007, 8, 2129.

(271) Fung, J.; Darabie, A. A.; McLaurin, J. Biochem. Biophys. Res. Commun. 2005, $328,1067$.

(272) Ariga, T.; Miyatake, T.; Yu, R. K. J. Neurosci. Res. 2010, 88, 2303.

(273) Valle-Delgado, J. J.; Alfonso-Prieto, M.; de Groot, N. S.; Ventura, S.; Samitier, J.; Rovira, C.; Fernandez-Busquets, X. FASEB J. 2010, 24, 4250. 
(274) Akaishi, T.; Morimoto, T.; Shibao, M.; Watanabe, S.; Sakai-Kato, K.;

Utsunomiya-Tate, N.; Abe, K. Neurosci. Lett. 2008, 444, 280.

(275) Kim, H.; Park, B. S.; Lee, K. G.; Choi, C. Y.; Jang, S. S.; Kim, Y. H.; Lee, S. E. J. Agric. Food Chem. 2005, 53, 8537.

(276) McLaurin, J.; Franklin, T.; Chakrabartty, A.; Fraser, P. E. J. Mol. Biol. 1998, $278,183$.

(277) McLaurin, J.; Golomb, R.; Jurewicz, A.; Antel, J. P.; Fraser, P. E. J. Biol. Chem. 2000, 275, 18495.

(278) Fenili, D.; Brown, M.; Rappaport, R.; McLaurin, J. J. Mol. Med. 2007, 85, 603. (279) (a) Palmer, A. M. Trends Pharmacol. Sci. 2011, 32, 141. (b) Gravitz, L. Nature 2011, 475, S9.

(280) Findeis, M. A.; Musso, G. M.; Arico-Muendel, C. C.; Benjamin, H. W.;

Hundal, A. M.; Lee, J. J.; Chin, J.; Kelley, M.; Wakefield, J.; Hayward, N. J.; Molineaux, S. M. Biochemistry 1999, 38, 6791.

(281) Lowe, T. L.; Strzelec, A.; Kiessling, L. L.; Murphy, R. M. Biochemistry 2001, $40,7882$.

(282) Pallitto, M. M.; Ghanta, J.; Heinzelman, P.; Kiessling, L. L.; Murphy, R. M. Biochemistry 1999, 38, 3570.

(283) Gibson, T. J.; Murphy, R. M. Biochemistry 2005, 44, 8898.

(284) Gordon, D. J.; Tappe, R.; Meredith, S. C. Journal of Peptide Research 2002, 60, 37.

(285) (a) Hughes, E.; Burke, R. M.; Doig, A. J. J. Biol. Chem. 2000, 275, 25109. (b) Kokkoni, N.; Stott, K.; Amijee, H.; Mason, J. M.; Doig, A. J. Biochemistry 2006, 45, 9906. 
(286) Austen, B. M.; Paleologou, K. E.; Ali, S. A. E.; Qureshi, M. M.; Allsop, D.; ElAgnaf, O. M. A. Biochemistry 2008, 47, 1984.

(287) Madine, J.; Wang, X.; Brown, D. R.; Middleton, D. A. ChemBioChem 2009, 10, 1982.

(288) Castelletto, V.; Cheng, G.; Hamley , I. W. Chem. Comm. 2011, 47, 12470.

(289) Watanabe, K.; Segawa, T.; Nakamura, K.; Kodaka, M.; Konakahara, T.; Okuno, H. Journal of Peptide Research 2001, 58, 342.

(290) Sundaram, R. K.; Kasinathan, C.; Stein, S.; Sundaram, P. Current Alzheimer Research 2008, 5, 26.

(291) (a) Rzepecki, P.; Schrader, T. J. Am. Chem. Soc. 2005, 127, 3016. (b)

Hochdörffer, K.; Marz-Berberich, J.; Nage-Steger, L.; Epple, M.; MeyerZaika, W.; Horn, A. H. C.; Stich, H.; Sinha, S.; Bitan, G.; Schrader, T. J. Am. Chem. Soc. 2011, 133, 4348.

(292) Sinha, S.; Lopes, D. H. J.; Du, Z. M.; Pang, E. S.; Shanmugam, A.; Lomakin, A.; Talbiersky, P.; Tennstaedt, A.; McDaniel, K.; Bakshi, R.; Kuo, P. Y.; Ehrmann, M.; Benedek, G. B.; Loo, J. A.; Klarner, F. G.; Schrader, T.; Wang, C. Y.; Bitan, G. J. Am. Chem. Soc. 2011, 133, 16958.

(293) Sato, J.; Takahashi, T.; Oshima, H.; Matsumura, S.; Mihara, H. Chem. Eur. J. 2007, 13, 7745 .

(294) Simons, M.; Keller, P.; De Strooper, B.; Beyreuther, K.; Dotti, C. G.; Simons, K. Proc. Natl. Acad. Sci. U. S. A. 1998, 95, 6460.

(295) Fonte, V.; Kapulkin, V.; Taft, A.; Fluet, A.; Friedman, D.; Link, C. D. Proc. Natl. Acad. Sci. U. S. A. 2002, 99, 9439.

(296) (a) Raman, B.; Ban, T.; Sakai, M.; Pasta, S. Y.; Ramakrishna, T.; Naiki, H.; Goto, Y.; Rao, C. M. Biochem. J. 2005, 392, 573. (b) Shammas, S. L.; 
Waudby, C. A.; Wang, S. Y.; Buell, A. K.; Knowles, T. P. J.; Ecroyd, H.;

Welland, M. E.; Carver, J. A.; Dobson, C. M.; Meehan, S. Biophys. J. 2011, $101,1681$.

(297) Calamini, B.; Silva, M. C.; Madoux, F.; Hutt, D. M.; Khanna, S.; Chalfant, M. A.; Saldanha, S. A.; Hodder, P.; Tait, B. D.; Garza, D.; Balch, W. E.; Morimoto, R. I. Nature Chemical Biology 2012, 8, 185.

(298) Yerbury, J. J.; Poon, S.; Meehan, S.; Thompson, B.; Kumita, J. R.; Dobson, C. M.; Wilson, M. R. FASEB J. 2007, 21, 2312.

(299) Chauhan, V. P. S.; Ray, I.; Chauhan, A.; Wisniewski, H. M. Biochem. Biophys. Res. Commun. 1999, 258, 241.

(300) Choo-Smith, L. P.; Garzon-Rodriguez, W.; Glabe, C. G.; Surewicz, W. K. J. Biol. Chem. 1997, 272, 22987.

(301) Matsuoka, Y.; Saito, M.; LaFrancois, J.; Saito, M.; Gaynor, K.; Olm, V.; Wang, L. L.; Casey, E.; Lu, Y. F.; Shiratori, C.; Lemere, C.; Duff, K. J. Neurosci. 2003, 23, 29.

(302) Schwarzman, A. L.; Tsiper, M.; Wente, H.; Wang, A.; Vitek, M. P.; Vasiliev, V.; Goldgaber, D. Amyloid-Journal of Protein Folding Disorders 2004, 11, 1. (303) Lauren, J.; Gimbel, D. A.; Nygaard, H. B.; Gilbert, J. W.; Strittmatter, S. M. Nature 2009, 457, 1128.

(304) Kudo, W.; Lee, H. P.; Zou, W. Q.; Wang, X. L.; Perry, G.; Zhu, X. W.; Smith, M. A.; Petersen, R. B.; Lee, H. G. Hum. Mol. Genet. 2012, 21, 1138.

(305) You, H. T.; Tsutsui, S.; Hameed, S.; Kannanayakal, T. J.; Chen, L. N.; Xia, P.; Engbers, J. D. T.; Lipton, S. A.; Stys, P. K.; Zamponi, G. W. Proc. Natl. Acad. Sci. U. S. A. 2012, 109, 1737. 
(306) Cabaleiro-Lago, C.; Quinlan-Pluck, F.; Lynch, I.; Dawson, K. A.; Linse, S. ACS Chem. Neurosci. 2010, 1, 279.

(307) Yoo, S. I.; Yang, M.; Brender, J. R.; Subramanian, V.; Sun, K.; Joo, N. E.; Jeong, S. H.; Ramamoorthy, A.; Kotov, N. A. Angew. Chem. Int. Ed. Engl. 2011, 50, 5110 .

(308) Geng, J.; Li, M.; Ren, J. S.; Wang, E. B.; Qu, X. G. Angew. Chem. Int. Ed. Engl. 2011, 50, 4184.

(309) Barandeh, F.; Nguyen, P.-L.; Kumar, R.; Iacobucci, G. J.; Kuznicki, M. L.; Kosterman, A.; Bergey, E. J.; Prasad, P. N.; Gunawardena, S. Plos One 2012, 7, e29424.

(310) Di, L.; Kerns, E. H.; Fan, K.; McConnell, O. J.; Carter, G. T. Eur. J. Med. Chem. 2003, 38, 223.

(311) Klementieva, O.; Benseny-Cases, N.; Gella, A.; Appelhans, D.; Voit, B.; Cladera, J. Biomacromolecules 2011, 12, 3903.

(312) Hong, L.; Koelsch, G.; Lin, X. L.; Wu, S. L.; Terzyan, S.; Ghosh, A. K.; Zhang, X. C.; Tang, J. Science 2000, 290, 150.

(313) (a) Kimura, T.; Shuto, D.; Kasai, S.; Liu, P.; Hidaka, K.; Hamada, T.; Hayashi, Y.; Hattori, C.; Asai, M.; Kitazume, S.; Saido, T. C.; Ishiura, S.; Kiso, Y. Bioorg. Med. Chem. Lett. 2004, 14, 1527. (b) Kimura, T.; Shuto, D.; Hamada, Y.; Igawa, N.; Kasai, S.; Liu, P.; Hidaka, K.; Hamada, T.; Hayashi, Y.; Kiso, Y. Bioorg. Med. Chem. Lett. 2005, 15, 211.

(314) Asai, M.; Hattori, C.; Iwata, N.; Saido, T. C.; Sasagawa, N.; Szabo, B.; Hashimoto, Y.; Maruyama, K.; Tanuma, S.; Kiso, Y.; Ishiura, S. J. Neurochem. 2006, 96, 533. 
(315) Rajendran, L.; Schneider, A.; Schlechtingen, G.; Weidlich, S.; Ries, J.; Braxmeier, T.; Schwille, P.; Schulz, J. B.; Schroeder, C.; Simons, M.; Jennings, G.; Knolker, H. J.; Simons, K. Science 2008, 320, 520.

(316) Parsons, R. B.; Subrarnaniam, D.; Austen, B. M. J. Neurochem. 2007, 102, 1276.

(317) Parsons, R. B.; Farrant, J. K.; Price, G. C.; Subramaniam, D.; Austen, B. M. Biochem. Soc. Trans. 2007, 35, 577.

(318) Parsons, R. B.; Austen, B. M. Biochem. Soc. Trans. 2007, 35, 974.

(319) Cai, H. B.; Wang, Y. S.; McCarthy, D.; Wen, H. J.; Borchelt, D. R.; Price, D. L.; Wong, P. C. Nature Neuroscience 2001, 4, 233.

(320) Geling, A.; Steiner, H.; Willem, M.; Bally-Cuif, L.; Haass, C. EMBO Rep. 2002, 3, 688 .

(321) (a) Weggen, S.; Eriksen, J. L.; Das, P.; Sagi, S. A.; Wang, R.; Pietrzik, C. U.; Findlay, K. A.; Smith, T. E.; Murphy, M. P.; Butler, T.; Kang, D. E.; Marquez-Sterling, N.; Golde, T. E.; Koo, E. H. Nature 2001, 414, 212. (b) Weggen, S.; Eriksen, J. L.; Sagi, S. A.; Pietrzik, C. U.; Ozols, V.; Fauq, A.; Golde, T. E.; Koo, E. H. J. Biol. Chem. 2003, 278, 31831. (c) Sagi, S. A.; Weggen, S.; Eriksen, J.; Golde, T. E.; Koo, E. H. J. Biol. Chem. 2003, 278, 31825. (d) Désiré, L.; Bourdin, J.; Loiseau, N.; Peillon, H.; Picard, V.; De Oliveira, C.; Bachelot, F.; Leblond, B.; Taverne, T.; Beausoleil, E.; Lacombe, S.; Drouin, D.; Schweighoffer, F. J. Biol. Chem. 2005, 280, 37516. (e) Kukar, T. L.; Ladd, T. B.; Bann, M. A.; Fraering, P. C.; Narlawar, R.; Maharvi, G. M.; Healy, B.; Chapman, R.; Welzel, A. T.; Price, R. W.; Moore, B.; Rangachari, V.; Cusack, B.; Eriksen, J.; Jansen-West, K.; Verbeeck, C.; Yager, D.; Eckman, C.; Ye, W. J.; Sagi, S.; Cottrell, B. A.; Torpey, J.; 
Rosenberry, T. L.; Fauq, A.; Wolfe, M. S.; Schmidt, B.; Walsh, D. M.; Koo, E. H.; Golde, T. E. Nature 2008, 453, 925.

(322) Morihara, T.; Chu, T.; Ubeda, O.; Beech, W.; Cole, G. M. J. Neurochem. 2002, 83,1009 .

(323) Lanz, T. A.; Karmilowicz, M. J.; Wood, K. M.; Pozdnyakov, N.; Du, P.;

Piotrowski, M. A.; Brown, T. M.; Nolan, C. E.; Richter, K. E. G.; Finley, J. E.; Fei, Q.; Ebbinghaus, C. F.; Chen, Y. L.; Spracklin, D. K.; Tate, B.;

Geoghegan, K. F.; Lau, L. F.; Auperin, D. D.; Schachter, J. B. J. Pharmacol. Exp. Ther. 2006, 319, 924.

(324) (a) Mayer, S. C.; Kreft, A. F.; Harrison, B.; Abou-Gharbia, M.; Antane, M.; Aschmies, S.; Atchison, K.; Chlenov, M.; Cole, D. C.; Comery, T.; Diamantidis, G.; Ellingboe, J.; Fan, K.; Galante, R.; Gonzales, C.; Ho, D. M.; Hoke, M. E.; Hu, Y.; Huryn, D.; Jain, U.; Jin, M.; Kremer, K.; Kubrak, D.; Lin, M.; Lu, P. M.; Magolda, R.; Martone, R.; Moore, W.; Oganesian, A.; Pangalos, M. N.; Porte, A.; Reinhart, P.; Resnick, L.; Riddell, D. R.; Sonnenberg-Reines, J.; Stock, J. R.; Sun, S. C.; Wagner, E.; Wang, T.; Woller, K.; Xu, Z.; Zaleska, M. M.; Zeldis, J.; Zhang, M. S.; Zhou, H.; Jacobsen, J. S. J. Med. Chem. 2008, 51, 7348. (b) Martone, R. L.; Zhou, H.; Atchison, K.; Comery, T.; Xu, J. Z.; Huang, X. Y.; Gong, X. H.; Jin, M.; Kreft, A.; Harrison, B.; Mayer, S. C.; Aschmies, S.; Gonzales, C.; Zaleska, M. M.; Riddell, D. R.; Wagner, E.; Lu, P. M.; Sun, S. C.; Sonnenberg-Reines, J.; Oganesian, A.; Adkins, K.; Leach, M. W.; Clarke, D. W.; Huryn, D.; AbouGharbia, M.; Magolda, R.; Bard, J.; Frick, G.; Raje, S.; Forlow, S. B.; Balliet, C.; Burczynski, M. E.; Reinhart, P. H.; Wan, H. I.; Pangalos, M. N.; Jacobsen, J. S. J. Pharmacol. Exp. Ther. 2009, 331, 598. 
(325) Serneels, L.; Van Biervliet, J.; Craessaerts, K.; Dejaegere, T.; Horre, K.; Van Houtvin, T.; Esselmann, H.; Paul, S.; Schafer, M. K.; Berezovska, O.; Hyman, B. T.; Sprangers, B.; Sciot, R.; Moons, L.; Jucker, M.; Yang, Z. X.; May, P. C.; Karran, E.; Wiltfang, J.; D'Hooge, R.; De Strooper, B. Science 2009, 324, 639.

(326) He, G.; Luo, W. J.; Li, P.; Remmers, C.; Netzer, W. J.; Hendrick, J.; Bettayeb, K.; Flajolet, M.; Gorelick, F.; Wennogle, L. P.; Greengard, P. Nature 2010, 467, 95 .

(327) (a) Schenk, D. Nat. Rev. Neurosci. 2002, 3, 824. (b) Birmingham, K.; Frantz, S. Nature Medicine 2002, 8, 199. (c) Schenk, D.; Hagen, M.; Seubert, P. Curr. Opin. Immunol. 2004, 16, 599.

(328) Hock, C.; Konietzko, U.; Streffer, J. R.; Tracy, J.; Signorell, A.; MullerTillmanns, B.; Lemke, U.; Henke, K.; Moritz, E.; Garcia, E.; Wollmer, M. A.; Umbricht, D.; de Quervain, D. J. F.; Hofmann, M.; Maddalena, A.; Papassotiropoulos, A.; Nitsch, R. M. Neuron 2003, 38, 547.

(329) Morgan, D.; Diamond, D. M.; Gottschall, P. E.; Ugen, K. E.; Dickey, C.; Hardy, J.; Duff, K.; Jantzen, P.; DiCarlo, G.; Wilcock, D.; Connor, K.; Hatcher, J.; Hope, C.; Gordon, M.; Arendash, G. W. Nature 2000, 408, 982.

(330) Orgogozo, J. M.; Gilman, S.; Dartigues, J. F.; Laurent, B.; Puel, M.; Kirby, L. C.; Jouanny, P.; Dubois, B.; Eisner, L.; Flitman, S.; Michel, B. F.; Boada, M.; Frank, A.; Hock, C. Neurology 2003, 61, 46.

(331) Gilman, S.; Koller, M.; Black, R. S.; Jenkins, L.; Griffith, S. G.; Fox, N. C.; Eisner, L.; Kirby, L.; Rovira, M. B.; Forette, F.; Orgogozo, J. M. Neurology 2005, 64, 1553. 
(332) Fox, N. C.; Black, R. S.; Gilman, S.; Rossor, M. N.; Griffith, S. G.; Jenkins, L.; Koller, M. Neurology 2005, 64, 1563.

(333) Holmes, C.; Boche, D.; Wilkinson, D.; Yadegarfar, G.; Hopkins, V.; Bayer, A.; Jones, R. W.; Bullock, R.; Love, S.; Neal, J. W.; Zotova, E.; Nicoll, J. A. R. Lancet 2008, 372, 216.

(334) Oddo, S.; Billings, L.; Kesslak, J. P.; Cribbs, D. H.; LaFerla, F. M. Neuron 2004, $43,321$.

(335) Eisele, Y. S.; Obermuller, U.; Heilbronner, G.; Baumann, F.; Kaeser, S. A.; Wolburg, H.; Walker, L. C.; Staufenbiel, M.; Heikenwalder, M.; Jucker, M. Science 2010, 330, 980.

(336) Sigurdsson, E. M.; Scholtzova, H.; Mehta, P. D.; Frangione, B.; Wisniewski, T. Am. J. Pathol. 2001, 159, 439.

(337) (a) Dickstein, D. L.; Biron, K. E.; Ujiie, M.; Pfeifer, C. G.; Jeffries, A. R.; Jefferies, W. A. FASEB J. 2006, 20, 426. (b) Dickstein, D. L.; Biron, K. E.; Ujiie, M.; Pfeifer, C. G.; Jeffries, A. R.; Jefferies, W. A. FASEB J. 2006, 20, 1573.

(338) Strazielle, N.; Ghersi-Egea, J. F.; Ghiso, J.; Dehouck, M. P.; Frangione, B.; Patlak, C.; Fenstermacher, J.; Gorevic, P. J. Neuropath. Exp. Neurol. 2000, 59, 29.

(339) (a) Salloway, S.; Sperling, R.; Gilman, S.; Fox, N. C.; Blennow, K.; Raskind, M.; Sabbagh, M.; Honig, L. S.; Doody, R.; van Dyck, C. H.; Mulnard, R.; Barakos, J.; Gregg, K. M.; Liu, E.; Lieberburg, I.; Schenk, D.; Black, R.; Grundman, M. Neurology 2009, 73, 2061. (b) Rinne, J. O.; Brooks, D. J.; Rossor, M. N.; Fox, N. C.; Bullock, R.; Klunk, W. E.; Mathis, C. A.; Blennow, K.; Barakos, J.; Okello, A. A.; de Llano, S. R. M.; Liu, E.; Koller, 
M.; Gregg, K. M.; Schenk, D.; Black, R.; Grundman, M. Lancet Neurol. 2010, $9,363$.

(340) http://www.clinicaltrials.gov/ct2/show/NCT00667810. In 2012.

(341) http://www.clinicaltrials.gov/ct2/show/NCT00905372. In 2012.

(342) Solomon, B.; Koppel, R.; Hanan, E.; Katzav, T. Proc. Natl. Acad. Sci. U. S. A. 1996, 93,452 .

(343) Dodart, J. C.; Bales, K. R.; Gannon, K. S.; Greene, S. J.; DeMattos, R. B.; Mathis, C.; DeLong, C. A.; Wu, S.; Wu, X.; Holtzman, D. M.; Paul, S. M. Nature Neuroscience 2002, 5, 452.

(344) Bard, F.; Cannon, C.; Barbour, R.; Burke, R. L.; Games, D.; Grajeda, H.; Guido, T.; Hu, K.; Huang, J. P.; Johnson-Wood, K.; Khan, K.; Kholodenko, D.; Lee, M.; Lieberburg, I.; Motter, R.; Nguyen, M.; Soriano, F.; Vasquez, N.; Weiss, K.; Welch, B.; Seubert, P.; Schenk, D.; Yednock, T. Nature Medicine 2000, 6, 916.

(345) Bacskai, B. J.; Kajdasz, S. T.; McLellan, M. E.; Games, D.; Seubert, P.; Schenk, D.; Hyman, B. T. J. Neurosci. 2002, 22, 7873.

(346) Bodin, K.; Ellmerich, S.; Kahan, M. C.; Tennent, G. A.; Loesch, A.; Gilbertson, J. A.; Hutchinson, W. L.; Mangione, P. P.; Gallimore, J. R.; Millar, D. J.; Minogue, S.; Dhillon, A. P.; Taylor, G. W.; Bradwell, A. R.; Petrie, A.; Gillmore, J. D.; Bellotti, V.; Botto, M.; Hawkins, P. N.; Pepys, M. B. Nature 2010, $468,93$.

(347) Pepys, M. B.; Herbert, J.; Hutchinson, W. L.; Tennent, G. A.; Lachmann, H. J.; Gallimore, J. R.; Lovat, L. B.; Bartfai, T.; Alanine, A.; Hertel, C.; Hoffmann, T.; Jakob-Roetne, R.; Norcross, R. D.; Kemp, J. A.; Yamamura, K.; Suzuki, 
M.; Taylor, G. W.; Murray, S.; Thompson, D.; Purvis, A.; Kolstoe, S.; Wood, S. P.; Hawkins, P. N. Nature 2002, 417, 254.

(348) Kolstoe, S. E.; Ridha, B. H.; Bellotti, V.; Wang, N.; Robinson, C. V.; Crutch, S. J.; Keir, G.; Kukkastenvehmas, R.; Gallimore, J. R.; Hutchinson, W. L.; Hawkins, P. N.; Wood, S. P.; Rossor, M. N.; Pepys, M. B. Proc. Natl. Acad. Sci. U. S. A. 2009, 106, 7619.

(349) Borman, S. Chemical and Engineering News 2010, Jan 25 issue, 30.

(350) (a) Bachurin, S.; Bukatina, E.; Lermontova, N.; Tkachenko, S.; Afanasiev, A.; Grigoriev, V.; Grigorieva, I.; Ivanov, Y.; Sablin, S.; Zefirov, N. In Annals of the New York Academy of Sciences. Neuroprotective agents: Fifth International Conference; New York Academy of Sciences \{a\} , 2 East 63rd Street, New York, NY, 10021, USA: 2001; Vol. 939, p 425. (b) Doody, R. S.; Gavrilova, S. I.; Sano, M.; Thomas, R. G.; Aisen, P. S.; Bachurin, S. O.; Seely, L.; Hung, D. Lancet 2008, 372, 207.

(351) Miller, G. Science 2010, 327, 1309.

(352) Cramer, P. E.; Cirrito, J. R.; Wesson, D. W.; Lee, C. Y. D.; Karlo, J. C.; Zinn, A. E.; Casali, B. T.; Restivo, J. L.; Goebel, W. D.; James, M. J.; Brunden, K. R.; Wilson, D. A.; Landreth, G. E. Science 2012, 335, 1503.

(353) (a) Inouye, H.; Fraser, P. E.; Kirschner, D. A. Biophys. J. 1993, 64, 502. (b) Serpell, L. C.; Blake, C. C. F.; Fraser, P. E. Biochemistry 2000, 39, 13269. (354) Nelson, R.; Eisenberg, D. Curr. Opin. Struct. Biol. 2006, 16, 260.

(355) Danielsson, J.; Jarvet, J.; Damberg, P.; Graslund, A. FEBS J 2005, 272, 3938. (356) (a) Barrow, C. J.; Zagorski, M. G. Science 1991, 253, 179. (b) Barrow, C. J.; Yasuda, A.; Kenny, P. T. M.; Zagorski, M. G. J. Mol. Biol. 1992, 225, 1075. 
(357) Shen, C.-L.; Scott, G. L.; Merchant, F.; Murphy, R. M. Biophys. J. 1993, 65, 2383.

(358) (a) Benzinger, T. L. S.; Gregory, D. M.; Burkoth, T. S.; Miller-Auer, H.; Lynn, D. G.; Botto, R. E.; Meredith, S. C. Proc. Natl. Acad. Sci. U. S. A. 1998, 95, 13407. (b) Benzinger, T. L. S.; Gregory, D. M.; Burkoth, T. S.; Miller-Auer, H.; Lynn, D. G.; Botto, R. E.; Meredith, S. C. Biochemistry 2000, 39, 3491.

(359) Antzutkin, O. N.; Leapman, R. D.; Balbach, J. J.; Tycko, R. Biochemistry 2002, $41,15436$.

(360) (a) Antzutkin, O. N.; Balbach, J. J.; Leapman, R. D.; Rizzo, N. W.; Reed, J.; Tycko, R. Proc. Natl. Acad. Sci. U. S. A. 2000, 97, 13045. (b) Petkova, A. T.; Ishii, Y.; Balbach, J. J.; Antzutkin, O. N.; Leapman, R. D.; Delaglio, F.; Tycko, R. Proc. Natl. Acad. Sci. U. S. A. 2002, 99, 16742. (c) Tycko, R. Biochemistry 2003, 42, 3151.

(361) Hilbich, C.; Kisterswoike, B.; Reed, J.; Masters, C. L.; Beyreuther, K. J. Mol. Biol. 1991, 218, 149.

(362) Kirschner, D. A.; Inouye, H.; Duffy, L. K.; Sinclair, A.; Lind, M.; Selkoe, D. J. Proc. Natl. Acad. Sci. U. S. A. 1987, 84, 6953.

(363) Ma, B. Y.; Nussinov, R. Proc. Natl. Acad. Sci. U. S. A. 2002, 99, 14126.

(364) Qiang, W.; Yau, W. M.; Tycko, R. J. Am. Chem. Soc. 2011, 133, 4018.

(365) Lührs, T.; Ritter, C.; Adrian, M.; Riek-Loher, D.; Bohrmann, B.; Döbeli, H.;

Schubert, D.; Riek, R. Proc. Natl. Acad. Sci. U. S. A. 2005, 102, 17342.

(366) Jang, H. B.; Zheng, J.; Lal, R.; Nussinov, R. Trends Biochem. Sci. 2008, 33, 91.

(367) (a) Cruz, L.; Urbanc, B.; Borreguero, J. M.; Lazo, N. D.; Teplow, D. B.;

Stanley, H. E. Proc. Natl. Acad. Sci. U. S. A. 2005, 102, 18258. (b)

Borreguero, J. M.; Urbanc, B.; Lazo, N. D.; Buldyrev, S. V.; Teplow, D. B.; 
Stanley, H. E. Proc. Natl. Acad. Sci. U. S. A. 2005, 102, 6015. (c) Baumketner, A.; Bernstein, S. L.; Wyttenbach, T.; Lazo, N. D.; Teplow, D. B.; Bowers, M. T.; Shea, J. E. Protein Science 2006, 15, 1239. (d) Teplow, D. B.; Lazo, N. D.; Bitan, G.; Bernstein, S.; Wyttenbach, T.; Bowers, M. T.; Baumketner, A.; Shea, J. E.; Urbanc, B.; Cruz, L.; Borreguero, J.; Stanley, H. E. Acc. Chem. Res. 2006, 39, 635. (e) Murray, M. M.; Krone, M. G.; Bernstein, S. L.; Baumketner, A.; Condron, M. M.; Lazo, N. D.; Teplow, D. B.; Wyttenbach, T.; Shea, J. E.; Bowers, M. T. J. Phys. Chem. B 2009, 113, 6041.

(368) Buchete, N. V.; Tycko, R.; Hummer, G. J. Mol. Biol. 2005, 353, 804. (369) Sikorski, P.; Atkins, E. D. T.; Serpell, L. C. Structure 2003, 11, 915.

(370) Serpell, L. C.; Smith, J. M. J. Mol. Biol. 2000, 299, 225.

(371) Pike, C. J.; Walencewiczwasserman, A. J.; Kosmoski, J.; Cribbs, D. H.; Glabe, C. G.; Cotman, C. W. J. Neurochem. 1995, 64, 253.

(372) Lansbury, P. T.; Costa, P. R.; Griffiths, J. M.; Simon, E. J.; Auger, M.; Halverson, K. J.; Kocisko, D. A. Nature Struct. Biol. 1995, 2, 990.

(373) Shao, H. Y.; Jao, S. C.; Ma, K.; Zagorski, M. G. J. Mol. Biol. 1999, 285, 755.

(374) Fletcher, T. G.; Keire, D. A. Protein Science 1997, 6, 666.

(375) Balbach, J. J.; Ishii, Y.; Antzutkin, O. N.; Leapman, R. D.; Rizzo, N. W.; Dyda, F.; Reed, J.; Tycko, R. Biochemistry 2000, 39, 13748.

(376) (a) Liang, Y.; Pingali, S. V.; Jogalekar, A. S.; Snyder, J. P.; Thiyagarajan, P.; Lynn, D. G. Biochemistry 2008, 47, 10018. (b) Mehta, A. K.; Lu, K.; Childers, W. S.; Liang, S.; Dong, J.; Snyder, J. P.; Pingali, S. V.; Thiyagarajan, P.; Lynn, D. G. J. Am. Chem. Soc. 2008, 130, 9829. 
(377) Colletier, J. P.; Laganowsky, A.; Landau, M.; Zhao, M. L.; Soriaga, A. B.; Goldschmidt, L.; Flot, D.; Cascio, D.; Sawaya, M. R.; Eisenberg, D. Proc. Natl. Acad. Sci. U. S. A. 2011, 108, 16938.

(378) Sawaya, M. R.; Sambashivan, S.; Nelson, R.; Ivanova, M. I.; Sievers, S. A.; Apostol, M. I.; Thompson, M. J.; Balbirnie, M.; Wiltzius, J. J. W.; McFarlane, H. T.; Madsen, A. O.; Riekel, C.; Eisenberg, D. Nature 2007, 447, 453.

(379) (a) Sachse, C.; Fandrich, M.; Grigorieff, N. Proc. Natl. Acad. Sci. U. S. A. 2008, 105, 7462. (b) Meinhardt, J.; Sachse, C.; Hortschansky, P.; Grigorieff, N.; Fandrich, M. J. Mol. Biol. 2009, 386, 869.

(380) Zhang, R.; Hu, X. Y.; Khant, H.; Ludtke, S. J.; Chiu, W.; Schmid, M. F.; Frieden, C.; Lee, J. M. Proc. Natl. Acad. Sci. U. S. A. 2009, 106, 4653. (381) Schmidt, M.; Sachse, C.; Richter, W.; Xu, C.; Fandrich, M.; Grigorieff, N. Proc. Natl. Acad. Sci. U. S. A. 2009, 106, 19813.

(382) Malinchik, S. B.; Inouye, H.; Szumowski, K. E.; Kirschner, D. A. Biophys. J. 1998, $74,537$.

(383) Perutz, M. F.; Finch, J. T.; Berriman, J.; Lesk, A. Proc. Natl. Acad. Sci. U. S. A. 2002, 99, 5591.

(384) Miller, Y.; Ma, B. Y.; Tsai, C. J.; Nussinov, R. Proc. Natl. Acad. Sci. U. S. A. 2010, 107, 14128.

(385) (a) Kad, N. M.; Thomson, N. H.; Smith, D. P.; Smith, D. A.; Radford, S. E. J. Mol. Biol. 2001, 313, 559. (b) Arnaudov, L. N.; de Vries, R. Biophys. J. 2005, 88,515 .

(386) Hou, L. M.; Shao, H. Y.; Zhang, Y. B.; Li, H.; Menon, N. K.; Neuhaus, E. B.; Brewer, J. M.; Byeon, I. J. L.; Ray, D. G.; Vitek, M. P.; Iwashita, T.; Makula, R. A.; Przybyla, A. B.; Zagorski, M. G. J. Am. Chem. Soc. 2004, 126, 1992. 
(387) Watson, A. A.; Fairlie, D. P.; Craik, D. J. Biochemistry 1998, 37, 12700.

(388) Narayanan, S.; Reif, B. Biochemistry 2005, 44, 1444.

(389) (a) Ma, B. Y.; Nussinov, R. Curr. Opin. Chem. Biol. 2006, 10, 445. (b) Miller, Y.; Ma, B.; Nussinov, R. Chem. Rev. 2010, 110, 4820.

(390) Urbanc, B.; Cruz, L.; Yun, S.; Buldyrev, S. V.; Bitan, G.; Teplow, D. B.; Stanley, H. E. Proc. Natl. Acad. Sci. U. S. A. 2004, 101, 17345.

(391) Baumketner, A.; Bernstein, S. L.; Wyttenbach, T.; Bitan, G.; Teplow, D. B.; Bowers, M. T.; Shea, J. E. Protein Science 2006, 15, 420.

(392) Zheng, J.; Jang, H.; Ma, B.; Tsai, C. J.; Nussinov, R. Biophys. J. 2007, 93, 3046.

(393) (a) Cheon, M.; Chang, I.; Mohanty, S.; Luheshi, L. M.; Dobson, C. M.; Vendruscolo, M.; Favrin, G. PLoS Computational Biology 2007, 3, 1727. (b) Cheon, M.; Favrin, G.; Chang, I.; Dobson, C. M.; Vendruscolo, M. Frontiers in Bioscience 2008, 13, 5614.

(394) Wei, G. H.; Jewett, A. I.; Shea, J. E. Phys. Chem. Chem. Phys. 2010, 12, 3622.

(395) Viet, M. H.; Ngo, S. T.; Lam, N. S.; Li, M. S. J. Phys. Chem. B 2011, 115, 7433.

(396) (a) Wang, Q.; Zhao, C.; Zhao, J.; Wang, J.; Yang, J.-C.; Yu, X.; Zheng, J. Langmuir 2010, 26, 3308. (b) Zhao, J.; Wang, Q.; Liang, G.; Zheng, J. Langmuir 2011, 27, 14876.

(397) Wang, Q.; Shah, N.; Zhao, J.; Wang, C.; Zhao, C.; Liu, L.; Li, L.; Zhou, F.; Zheng, J. Phys. Chem., Chem. Phys. 2011, 13, 15200.

(398) Lomakin, A.; Teplow, D. B.; Kirschner, D. A.; Benedek, G. B. Proc. Natl. Acad. Sci. U. S. A. 1997, 94, 7942. 
(399) (a) Walsh, D. M.; Lomakin, A.; Benedek, G. B.; Condron, M. M.; Teplow, D. B. J. Biol. Chem. 1997, 272, 22364. (b) Caughey, B.; Lansbury, P. T. Annual Review of Neuroscience 2003, 26, 267.

(400) Lomakin, A.; Chung, D. S.; Benedek, G. B.; Kirschner, D. A.; Teplow, D. B. Proc. Natl. Acad. Sci. U. S. A. 1996, 93, 1125.

(401) Israelachvili, J. N. Intermolecular and Surface Forces; Academic Press: San Diego, 1991.

(402) Hamley, I. W. Angew. Chem. 2007, 46, 8128.

(403) (a) Asakura, S.; Oosawa, F. J. Chem. Phys. 1954, 22, 1255. (b) Oosawa, F.; Asakura, S. Thermodynamics of the polymerization of protein; Academic Press: New York, 1975.

(404) (a) Knowles, T. P. J.; Waudby, C. A.; Devlin, G. L.; Cohen, S. I. A.; Aguzzi, A.; Vendruscolo, M.; Terentjev, E. M.; Welland, M. E.; Dobson, C. M. Science 2009, 326, 1533. (b) Cohen, S. I. A.; Vendruscolo, M.; Welland, M. E.; Dobson, C. M.; Terentjev, E. M.; Knowles, T. P. J. J. Chem. Phys. 2011, 135. (c) Cohen, S. I. A.; Vendruscolo, M.; Dobson, C. M.; Knowles, T. P. J. J. Chem. Phys. 2011, 135. (d) Cohen, S. I. A.; Vendruscolo, M.; Dobson, C. M.; Knowles, T. P. J. J. Chem. Phys. 2011, 135.

(405) (a) Andreu, J. M.; Timasheff, S. N. Methods Enzymol. 1986, 130, 47. (b) Johan, K.; Westermark, G.; Engström, U.; Gustavsson, A.; Hultman, P.; Westermark, P. Proc. Natl. Acad. Sci. U. S. A. 1998, 95, 2558. (c) Baskakov, I. V.; Legname, G.; Gryczynski, Z.; Prusiner, S. B. Protein Science 2004, 13, 586. (406) Ha, C.; Park, C. B. Langmuir 2006, 22, 6977.

(407) Lin, M. S.; Chen, L. Y.; Tsai, H. T.; Wang, S. S. S.; Chang, Y.; Higuchi, A.; Chen, W. Y. Langmuir 2008, 24, 5802. 
(408) Hellstrand, E.; Boland, B.; Walsh, D. M.; Linse, S. ACS Chem. Neurosci. 2010, $1,13$.

(409) Wang, S.; Ishii, Y. J. Am. Chem. Soc. 2012, 134, 2848.

(410) Petkova, A. T.; Leapman, R. D.; Guo, Z. H.; Yau, W. M.; Mattson, M. P.; Tycko, R. Science 2005, 307, 262.

(411) Fawzi, N. L.; Ying, J. F.; Ghirlando, R.; Torchia, D. A.; Clore, G. M. Nature 2011, 480, 268.

(412) (a) Esler, W. P.; Stimson, E. R.; Ghilardi, J. R.; Vinters, H. V.; Lee, J. P.; Mantyh, P. W.; Maggio, J. E. Biochemistry 1996, 35, 749. (b) Naiki, H.; Nakakuki, K. Lab. Invest. 1996, 74, 374.

(413) Kusumoto, Y.; Lomakin, A.; Teplow, D. B.; Benedek, G. B. Proc. Natl. Acad. Sci. U. S. A. 1998, 95, 12277.

(414) Murphy, R. M.; Pallitto, M. R. Journal of Structural Biology 2000, 130, 109.

(415) Shen, C.-L.; Fitzgerald, M. C.; Murphy, R. M. Biophys. J. 1994, 67, 1238.

(416) (a) Knowles, T. P.; Shu, W.; Devlin, G. L.; Meehan, S.; Auer, S.; Dobson, C. M.; Welland, M. E. Proc. Natl. Acad. Sci. U. S. A. 2007, 104, 10016. (b) Kotarek, J. A.; Johnson, K. C.; Moss, M. A. Anal. Biochem. 2008, 378, 15.

(417) Cannon, M. J.; Williams, A. D.; Wetzel, R.; Myszka, D. G. Anal. Biochem. 2004, 328, 67.

(418) (a) Ha, C.; Park, C. B. Langmuir 2006, 22, 6977. (b) Ha, C.; Ryu, J.; Park, C. B. Biochemistry 2007, 46, 6118.

(419) Sánchez, L.; Madurga, S.; Pukala, T.; Vilaseca, M.; Lopez-Iglesias, C.; Robinson, C. V.; Giralt, E.; Carulla, N. J. Am. Chem. Soc. 2011, 133, 6505. (420) O'Nuallain, B.; Shivaprasad, S.; Kheterpal, I.; Wetzel, R. Biochemistry 2005, 44, 12709. 
(421) Inouye, H.; Kirschner, D. A. Journal of Structural Biology 2000, 130, 123.

(422) Wood, S. J.; Maleef, B.; Hart, T.; Wetzel, R. J. Mol. Biol. 1996, 256, 870.

(423) Wetzel, R.; Shivaprasad, S.; Williams, A. D. Biochemistry 2007, 46, 1.

(424) Williams, A. D.; Shivaprasad, S.; Wetzel, R. J. Mol. Biol. 2006, 357, 1283.

(425) Baldwin, A. J.; Knowles, T. P. J.; Tartaglia, G. G.; Fitzpatrick, A. W.; Devlin, G. L.; Shammas, S. L.; Waudby, C. A.; Mossuto, M. F.; Meehan, S.; Gras, S. L.; Christodoulou, J.; Anthony-Cahill, S. J.; Barker, P. D.; Vendruscolo, M.; Dobson, C. M. J. Am. Chem. Soc. 2011, 133, 14160.

(426) Jahn, T. R.; Radford, S. E. FEBS J 2005, 272, 5962.

(427) Chiti, F.; Webster, P.; Taddei, N.; Clark, A.; Stefani, M.; Ramponi, G.; Dobson, C. M. Proc. Natl. Acad. Sci. U. S. A. 1999, 96, 3590.

(428) (a) Krebs, M. R. H.; Wilkins, D. K.; Chung, E. W.; Pitkeathly, M. C.; Chamberlain, A. K.; Zurdo, J.; Robinson, C. V.; Dobson, C. M. J. Mol. Biol. 2000, 300, 541. (b) Balbirnie, M.; Grothe, R.; Eisenberg, D. S. Proc. Natl. Acad. Sci. U. S. A. 2001, 98, 2375. (c) Krebs, M. R. H.; Morozova-Roche, L. A.; Daniel, K.; Robinson, C. V.; Dobson, C. M. Protein Science 2004, 13, 1933.

(429) Gaughey, B.; Baron, G. S. Nature (London) 2006, 443, 803.

(430) Fraser, P. E.; Nguyen, J. T.; Inouye, H.; Surewicz, W. K.; Selkoe, D. J.; Podlisny, M. B.; Kirschner, D. A. Biochemistry 1992, 31, 10716.

(431) Kim, W.; Hecht, M. H. J. Biol. Chem. 2005, 280, 35069.

(432) Kim, W. K.; Hecht, M. H. Proc. Natl. Acad. Sci. U. S. A. 2006, 103, 15824.

(433) Wurth, C.; Guimard, N. K.; Hecht, M. H. J. Mol. Biol. 2002, 319, 1279.

(434) Madine, J.; Davies, H. A.; Shaw, C.; Hamley, I. W.; Middleton, D. A. Chem. Comm. 2012, 48, 2976. 
(435) Pronchik, J.; He, X.; Giurleo, J. T.; Talaga, D. S. J. Am. Chem. Soc. 2010, 132, 9797.

(436) Fernandez-Escamilla, A. M.; Rousseau, F.; Schymkowitz, J.; Serrano, L. Nature Biotechnology 2004, 22, 1302.

(437) Pawar, A. P.; DuBay, K. F.; Zurdo, J.; Chiti, F.; Vendruscolo, M.; Dobson, C. M. J. Mol. Biol. 2005, 350, 379.

(438) Scheidt, H. A.; Morgado, I.; Rothemund, S.; Huster, D.; Fandrich, M. Angew. Chem. Int. Ed. Engl. 2011, 50, 2837.

(439) Hilbich, C.; Kisterswoike, B.; Reed, J.; Masters, C. L.; Beyreuther, K. J. Mol. Biol. 1992, 228, 460.

(440) (a) Tjernberg, L. O.; Naslund, J.; Lindqvist, F.; Johansson, J.; Karlstrom, A. R.; Thyberg, J.; Terenius, L.; Nordstedt, C. J. Biol. Chem. 1996, 271, 8545. (b) Tjernberg, L. O.; Lilliehook, C.; Callaway, D. J. E.; Naslund, J.; Hahne, S.; Thyberg, J.; Terenius, L.; Nordstedt, C. J. Biol. Chem. 1997, 272, 12601.

(441) Chafekar, S. M.; Malda, H.; Merkx, M.; Meijer, E. W.; Viertl, D.; Lashuel, H. A.; Baas, F.; Scheper, W. ChemBioChem 2007, 8, 1857.

(442) Reinke, A. A.; Ung, P. M. U.; Quintero, J. J.; Carlson, H. A.; Gestwicki, J. E. J. Am. Chem. Soc. 2010, 132, 17655.

(443) Tjernberg, L. O.; Callaway, D. J. E.; Tjernberg, A.; Hahne, S.; Lilliehöök, C.; Terenius, L.; Thyberg, J.; Nordstedt, C. J. Biol. Chem. 1999, 274, 12619.

(444) Krysmann, M. J.; Castelletto, V.; Kelarakis, A.; Hamley , I. W.; Hule, R. A.; Pochan, D. J. Biochemistry 2008, 47, 4597.

(445) Gordon, D. J.; Sciarretta, K. L.; Meredith, S. C. Biochemistry 2001, 40, 8237.

(446) Soto, C.; Sigurdsson, E. M.; Morelli, L.; Kumar, R. A.; Castaño, E. M.; Frangione, B. Nature Medicine 1998, 4, 822. 
(447) Wood, S. J.; Wetzel, R.; Martin, J. D.; Hurle, M. R. Biochemistry 1995, 34, 724.

(448) Chacon, M. A.; Barria, M. I.; Soto, C.; Inestrosa, N. C. Molecular Psychiatry 2004, 9, 953.

(449) Zhang, G.; Leibowitz, M. J.; Sinko, P. J.; Stein, S. Bioconjugate Chemistry 2003, 14, 86.

(450) (a) Lu, K.; Jacob, J.; Thiyagarajan, P.; Conticello, V. P.; Lynn, D. G. J. Am. Chem. Soc. 2003, 125, 6391. (b) Dong, J.; Lu, K.; Lakdawala, A.; Mehta, A. K.; Lynn, D. G. Amyloid 2006, 13, 206.

(451) Childers, W. S.; Mehta, A. K.; Lu, K.; Lynn, D. G. J. Am. Chem. Soc. 2009, 131,10165 .

(452) Fernandez-Escamilla, A.-M.; Rousseau, F.; Schymkowitz, J.; Serrano, L. Nature Biotechnology 2004, 22, 1302.

(453) Kallberg, Y.; Gustafsson, M.; Persson, B.; Thyberg, J.; Johansson, J. J. Biol. Chem. 2001, 276, 12945.

(454) Sticht, H.; Bayer, P.; Willbold, D.; Dames, S.; Hilbich, C.; Beyreuther, K.; Frank, R. W.; Rosch, P. Eur. J. Biochem. 1995, 233, 293.

(455) Coles, M.; Bicknell, W.; Watson, A. A.; Fairlie, D. P.; Craik, D. J. Biochemistry 1998, 37, 11064.

(456) Naskar, J.; Drew, M. G. B.; Deb, I.; Das, S.; Banerjee, A. Org. Lett. 2008, 10, 2625.

(457) Tekirian, T. L. Journal of Alzheimer's Disease 2001, 3, 241.

(458) Hosoda, R.; Saido, T. C.; Otvos, L.; Arai, T.; Mann, D. M. A.; Lee, V. M. Y.; Trojanowski, J. Q.; Iwatsubo, T. J. Neuropath. Exp. Neurol. 1998, 57, 1089. 
(459) (a) He, W. L.; Barrow, C. J. Biochemistry 1999, 38, 10871. (b) Schilling, S.; Lauber, T.; Schaupp, M.; Manhart, S.; Scheel, E.; Bohm, G.; Demuth, H. U. Biochemistry 2006, 45, 12393.

(460) Halverson, K.; Fraser, P. E.; Kirschner, D. A.; Lansbury, P. T. Biochemistry 1990, 29, 2639.

(461) Soreghan, B.; Kosmoski, J.; Glabe, C. G. J. Biol. Chem. 1994, 269, 28551.

(462) Sabate, R.; Estelrich, J. J. Phys. Chem. B 2005, 109, 11027.

(463) Kim, J.; Lee, M. Biochem. Biophys. Res. Commun. 2004, 316, 393.

(464) Yong, W.; Lomakin, A.; Kirkitadze, M. D.; Teplow, D. B.; Chen, S. H.;

Benedek, G. B. Proc. Natl. Acad. Sci. U. S. A. 2002, 99, 150.

(465) Rangachari, V.; Moore, B. D.; Reed, D. K.; Sonoda, L. K.; Bridges, A. W.;

Conboy, E.; Hartigan, D.; Rosenberry, T. L. Biochemistry 2007, 46, 12451.

(466) Lin, J. M.; Lin, T. L.; Jeng, U. S.; Huang, Z. H.; Huang, Y. S. Soft Matter 2009, $5,3913$.

(467) Sureshbabu, N.; Kirubagaran, R.; Jayakumar, R. Eur. Biophys. J. Biophys. Lett. 2009, 38, 355 .

(468) Wood, S. J.; MacKenzie, L.; Maleeff, B.; Hurle, M. R.; Wetzel, R. J. Biol. Chem. 1996, 271, 4086.

(469) He, C. Q.; Hou, Y. B.; Han, Y. C.; Wang, Y. L. Langmuir 2011, 27, 4551.

(470) Hamill, A. C.; Lee, C. T. J. Phys. Chem. B 2009, 113, 6164.

(471) Han, Y.; Huang, X.; Cao, M.; Wang, Y. J. Phys. Chem. B 2008, 112, 15195.

(472) (a) Michikawa, M. J. Neurosci. Res. 2003, 72, 141. (b) Michikawa, M. Mol. Neurobiol. 2003, 27, 1.

(473) Gorbenko, G. P.; Kinnunen, P. K. J. Chem. Phys. Lipids 2006, 141, 72. 
(474) (a) Kawarabayashi, T.; Shoji, M.; Younkin, L. H.; Lin, W. L.; Dickson, D. W.; Murakami, T.; Matsubara, E.; Abe, K.; Ashe, K. H.; Younkin, S. G. J. Neurosci. 2004, 24, 3801. (b) Kim, S. I.; Yi, J. S.; Ko, Y. G. J. Cell. Biochem. 2006, 99, 878. (c) Okada, T.; Ikeda, K.; Wakabayashi, M.; Ogawa, M.; Matsuzaki, K. J. Mol. Biol. 2008, 382, 1066.

(475) Mandal, P. K.; Pettegrew, J. W. Neurochem. Res. 2004, 29, 2267.

(476) Morita, M.; Hamada, T.; Tendo, Y.; Hata, t.; Vestergaard, M. C.; Takagi, M. Soft Matter 2012, 8, 2816.

(477) (a) Matsuzaki, K. BBA Biomembranes 2007, 1768, 1935. (b) Aisenbrey, C.; Borowik, T.; Bystrom, R.; Bokvist, M.; Lindstrom, F.; Misiak, H.; Sani, M. A.; Grobner, G. Eur. Biophys. J. Biophys. Lett. 2008, 37, 247.

(478) Ma, K.; Clancy, E. L.; Zhang, Y. B.; Ray, D. G.; Wollenberg, K.; Zagorski, M. G. J. Am. Chem. Soc. 1999, 121, 8698.

(479) (a) Ege, C.; Lee, K. Y. C. Biophys. J. 2004, 87, 1732. (b) Kremer, J. J.; Sklansky, D. J.; Murphy, R. M. Biochemistry 2001, 40, 8563.

(480) Terzi, E.; Holzemann, G.; Seelig, J. Biochemistry 1997, 36, 14845.

(481) Chi, E. Y.; Ege, C.; Winans, A.; Majewski, J.; Wu, G. H.; Kjaer, K.; Lee, K. Y. C. Proteins 2008, 72, 1 .

(482) Wong, P. T.; Schauerte, J. A.; Wisser, K. C.; Ding, H.; Lee, E. L.; Steel, D. G.; Gafni, A. J. Mol. Biol. 2009, 386, 81.

(483) Terzi, E.; Holzemann, G.; Seelig, J. Biochemistry 1994, 33, 7434.

(484) Martins, I. C.; Kuperstein, I.; Wilkinson, H.; Maes, E.; Vanbrabant, M.; Jonckheere, W.; Van Gelder, P.; Hartmann, D.; D'Hooge, R.; De Strooper, B.; Schymkowitz, J.; Rousseau, F. EMBO J. 2008, 27, 224. 
(485) Kawahara, M.; Kuroda, Y.; Arispe, N.; Rojas, E. J. Biol. Chem. 2000, 275, 14077.

(486) McLaurin, J.; Chakrabartty, A. J. Biol. Chem. 1996, 271, 26482.

(487) Williams, T. L.; Day, I. J.; Serpell, L. C. Langmuir 2010, 26, 17260.

(488) Jang, H.; Zheng, J.; Nussinov, R. Biophys. J. 2007, 93, 1938.

(489) Strodel, B.; Lee, J. W. L.; Whittleston, C. S.; Wales, D. J. J. Am. Chem. Soc. 2010, 132,13300 .

(490) Davis, C. H.; Berkowitz, M. L. Biophys. J. 2009, 96, 785.

(491) Kremer, J. J.; Pallitto, M. M.; Sklansky, D. J.; Murphy, R. M. Biochemistry 2000, 39, 10309.

(492) McLaurin, J.; Franklin, T.; Fraser, P. E.; Chakrabartty, A. J. Biol. Chem. 1998, $273,4506$.

(493) (a) Matsuzaki, K.; Horikiri, C. Biochemistry 1999, 38, 4137. (b) Okada, T.; Wakabayashi, M.; Ikeda, K.; Matsuzaki, K. J. Mol. Biol. 2007, 371, 481. (494) Wood, S. J.; Maleeff, B.; Hart, T.; Wetzel, R. J. Mol. Biol. 1996, 256, 870. (495) Choo-Smith, L. P.; Surewicz, W. K. FEBS Lett. 1997, 402, 95.

(496) (a) Yip, C. M.; Elton, E. A.; Darabie, A. A.; Morrison, M. R.; McLaurin, J. J. Mol. Biol. 2001, 311, 723. (b) Yip, C. M.; Darabie, A. A.; McLaurin, J. J. Mol. Biol. 2002, 318, 97.

(497) Bokvist, M.; Lindstrom, F.; Watts, A.; Grobner, G. J. Mol. Biol. 2004, 335, 1039.

(498) Chauhan, A.; Ray, I.; Chauhan, V. P. S. Neurochem. Res. 2000, 25, 423.

(499) Hellstrand, E.; Sparr, E.; Linse, S. Biophys. J. 2010, 98, 2206.

(500) Calamai, M.; Pavone, F. S. J. Am. Chem. Soc. 2011, 133, 12001.

(501) Dante, T.; Hauss, T.; Dencher, N. A. Biophys. J. 2002, 83, 2610. 
(502) Maltseva, E.; Kerth, A.; Blume, A.; Mohwald, H.; Brezesinski, G. Chembiochem 2005, 6, 1817.

(503) Buchsteiner, A.; Hauss, T.; Dencher, N. A. Soft Matter 2011, 8, 424.

(504) Ravault, S.; Flore, C.; Saurel, O.; Milon, A.; Brasseur, R.; Lins, L. Langmuir 2009, 25, 10948

(505) Kowalewski, T.; Holtzman, D. M. Proc. Natl. Acad. Sci. U. S. A. 1999, 96, 3688

(506) Austen, B.; Christodoulou, G.; Terry, J. E. The journal of nutrition, health \& aging 2002, 6, 377.

(507) Qiu, L. M.; Lewis, A.; Como, J.; Vaughn, M. W.; Huang, J. Y.; Somerharju, P.; Virtanen, J.; Cheng, K. H. Biophys. J. 2009, 96, 4299.

(508) Curtain, C. C.; Ali, F. E.; Smith, D. G.; Bush, A. I.; Masters, C. L.; Barnham, K. J. J. Biol. Chem. 2003, 278, 2977.

(509) Mason, R. P.; Shoemaker, W. J.; Shajenko, L.; Chambers, T. E.; Herbette, L. G. Neurobiology of Aging 1992, 13, 413.

(510) Fantini, J.; Yahi, N. Expert Rev. Mol. Med. 2010, 12, e27.

(511) Locatelli, S.; Lutjohann, D.; Schmidt, H. H. J.; Otto, C.; Beisiegel, U.; von Bergmann, K. Arch. Neurol. 2002, 59, 213.

(512) Harris, J. R. Micron 2002, 33, 609.

(513) Kakio, A.; Nishimoto, S.; Yanagisawa, K.; Kozutsumi, Y.; Matsuzaki, K. J. Biol. Chem. 2001, 276, 24985.

(514) Zhang, Q. H.; Powers, E. T.; Nieva, J.; Huff, M. E.; Dendle, M. A.; Bieschke, J.; Glabe, C. G.; Eschenmoser, A.; Wentworth, P.; Lerner, R. A.; Kelly, J. W. Proc. Natl. Acad. Sci. U. S. A. 2004, 101, 4752. 
(515) Bieschke, J.; Zhang, Q. H.; Powers, E. T.; Lerner, R. A.; Kelly, J. W. Biochemistry 2005, 44, 4977.

(516) (a) Bush, A. I. Curr. Opin. Chem. Biol. 2000, 4, 184. (b) Metal ions and neurodegenerative disorders; Zatta, P., Ed.; World Scientific: Singapore, 2003. (c) Adlard, P. A.; Bush, A. I. J. Alzheimers Dis. 2006, 10, 145. (d) Miu, A. C.; Benga, O. J. Alzheimers Dis. 2006, 10, 179.

(517) (a) Smith, M. A.; Harris, P. L. R.; Sayre, L. M.; Perry, G. Proc. Natl. Acad. Sci. U. S. A. 1997, 94, 9866. (b) Sayre, L. M.; Perry, G.; Smith, M. A. Curr. Opin. Chem. Biol. 1999, 3, 220. (c) Sayre, L. M.; Perry, G.; Harris, P. L. R.; Liu, Y. H.; Schubert, K. A.; Smith, M. A. J. Neurochem. 2000, 74, 270. (d) Lovell, M. A.; Robertson, J. D.; Teesdale, W. J.; Campbell, J. L.; Markesbery, W. R. J. Neurol. Sci. 1998, 158, 47. (e) Suh, S. W.; Jensen, K. B.; Jensen, M. S.; Silva, D. S.; Kesslak, P. J.; Danscher, G.; Frederickson, C. J. Brain Res. 2000, 852, 274.

(518) (a) Bush, A. I.; Pettingell, W. H.; Paradis, M. D.; Tanzi, R. E. J. Biol. Chem. 1994, 269, 12152. (b) Atwood, C. S.; Moir, R. D.; Huang, X. D.; Scarpa, R. C.; Bacarra, N. M. E.; Romano, D. M.; Hartshorn, M. K.; Tanzi, R. E.; Bush, A. I. J. Biol. Chem. 1998, 273, 12817.

(519) Hu, W. P.; Chang, G. L.; Chen, S. J.; Kuo, Y. M. J. Neurosci. Methods 2006, $154,190$.

(520) Rottkamp, C. A.; Raina, A. K.; Zhu, X. W.; Gaier, E.; Bush, A. I.; Atwood, C. S.; Chevion, M.; Perry, G.; Smith, M. A. Free Radical Biology and Medicine 2001, 30, 447.

(521) (a) Liu, S. T.; Howlett, G.; Barrow, C. J. Biochemistry 1999, 38, 9373. (b) Curtain, C. C.; Ali, F.; Volitakis, I.; Cherny, R. A.; Norton, R. S.; Beyreuther, 
K.; Barrow, C. J.; Masters, C. L.; Bush, A. I.; Barnham, K. J. J. Biol. Chem. 2001, 276, 20466. (c) Syme, C. D.; Nadal, R. C.; Rigby, S. E. J.; Viles, J. H. J. Biol. Chem. 2004, 279, 18169. (d) Miura, T.; Suzuki, K.; Kohata, N.;

Takeuchi, H. Biochemistry 2000, 39, 7024. (e) Nakamura, M.; Shishido, N.; Nunomura, A.; Smith, M. A.; Perry, G.; Hayashi, Y.; Nakayama, K.; Hayashi, T. Biochemistry 2007, 46, 12737. (f) Dong, J. J.; Shokes, J. E.; Scott, R. A.; Lynn, D. G. J. Am. Chem. Soc. 2006, 128, 3540. (g) Dong, J.; Canfield, J. M.; Mehta, A. K.; Shokes, J. E.; Tian, B.; Childers, W. S.; Simmons, J. A.; Mao, Z.; Scott, R. A.; Warncke, K.; Lynn, D. G. Proc. Natl. Acad. Sci. U. S. A. 2007, 104, 13313.

(522) Streltsov, V. A.; Titmuss, S. J.; Epa, V. C.; Barnham, K. J.; Masters, C. L.; Varghese, J. N. Biophys. J. 2008, 95, 3447.

(523) Parthasarathy, S.; Long, F.; Miller, Y.; Xiao, Y. L.; McElheny, D.; Thurber, K.; Ma, B. Y.; Nussinov, R.; Ishii, Y. J. Am. Chem. Soc. 2011, 133, 3390.

(524) Mantyh, P. W.; Ghilardi, J. R.; Rogers, S.; Demaster, E.; Allen, C. J.; Stimson, E. R.; Maggio, J. E. J. Neurochem. 1993, 61, 1171.

(525) Pedersen, J. T.; Teilum, K.; Heegaard, N. H. H.; Ostergaard, J.; Adolph, H. W.; Hemmingsen, L. Angew. Chem. Int. Ed. Engl. 2011, 50, 2532.

(526) Garai, K.; Sengupta, P.; Sahoo, B.; Maiti, S. Biochem. Biophys. Res. Commun. 2006, 345, 210.

(527) Innocenti, M.; Salvietti, E.; Guidotti, M.; Casini, A.; Bellandi, S.; Foresti, M. L.; Gabbiani, C.; Pozzi, A.; Zatta, P.; Messori, L. J. Alzheimers Dis. 2010, 19, 1323.

(528) Ryu, J.; Girigoswami, K.; Ha, C.; Ku, S. H.; Park, C. B. Biochemistry 2008, 47, 5328. 
(529) (a) Gupta, V. B.; Anitha, S.; Hegde, M. L.; Zecca, L.; Garruto, R. M.; Ravid, R.; Shankar, S. K.; Stein, R.; Shanmugavelu, P.; Rao, K. S. J. Cell. Mol. Life Sci. 2005, 62, 143. (b) Walton, J. R. Neurotoxicology 2006, 27, 385. (c) Zatta, P. J. Alzheimers Dis. 2006, 10, 33.

(530) Candy, J. M.; Oakley, A. E.; Klinowski, J.; Carpenter, T. A.; Perry, R. H.; Atack, J. R.; Perry, E. K.; Blessed, G.; Fairbairn, A.; Edwardson, J. A. Lancet 1986, 1,354 .

(531) Good, P. F.; Perl, D. P.; Bierer, L. M.; Schmeidler, J. Ann. Neurol. 1992, 31, 286.

(532) (a) Ricchelli, F.; Drago, D.; Filippi, B.; Tognon, G.; Zatta, P. Cell. Mol. Life Sci. 2005, 62, 1724. (b) Drago, D.; Bettella, M.; Bolognin, S.; Cendron, L.; Scancar, J.; Milacic, R.; Ricchelli, F.; Casini, A.; Messori, L.; Tognon, G.; Zatta, P. Int. J. Biochem. Cell Biol. 2008, 40, 731.

(533) (a) Bondy, S. C.; Guo-Ross, S. X.; Truong, A. T. Brain Res. 1998, 799, 91. (b) Huang, X. D.; Atwood, C. S.; Hartshorn, M. A.; Multhaup, G.; Goldstein, L. E.; Scarpa, R. C.; Cuajungco, M. P.; Gray, D. N.; Lim, J.; Moir, R. D.; Tanzi, R. E.; Bush, A. I. Biochemistry 1999, 38, 7609. (c) Huang, X. D.; Cuajungco, M. P.; Atwood, C. S.; Hartshorn, M. A.; Tyndall, J. D. A.; Hanson, G. R.; Stokes, K. C.; Leopold, M.; Multhaup, G.; Goldstein, L. E.; Scarpa, R. C.; Saunders, A. J.; Lim, J.; Moir, R. D.; Glabe, C.; Bowden, E. F.; Masters, C. L.; Fairlie, D. P.; Tanzi, R. E.; Bush, A. I. J. Biol. Chem. 1999, 274, 37111. (534) Schubert, D.; Chevion, M. Biochem. Biophys. Res. Commun. 1995, $216,702$.

(535) Liu, B.; Moloney, A.; Meehan, S.; Morris, K.; Thomas, S. E.; Serpell, L. C.; Hider, R.; Marciniak, S. J.; Lomas, D. A.; Crowther, D. C. J. Biol. Chem. 2011, 286, 4248 . 
(536) (a) Bush, A. I.; Multhaup, G.; Moir, R. D.; Williamson, T. G.; Small, D. H.; Rumble, B.; Pollwein, P.; Beyreuther, K.; Masters, C. L. J. Biol. Chem. 1993, 268, 16109. (b) Multhaup, G.; Bush, A. I.; Pollwein, P.; Masters, C. L. FEBS Lett. 1994, 355, 151. (c) Multhaup, G.; Ruppert, T.; Schlicksupp, A.; Hesse, L.; Bill, E.; Pipkorn, R.; Masters, C. L.; Beyreuther, K. Biochemistry 1998, 37, 7224.

(537) (a) Vandermeulen, G. W. M.; Tziatzios, C.; Klok, H.-A. Macromolecules 2003, 36, 4107. (b) Vandermeulen, G. W. M.; Klok, H.-A. Macromol. Biosci. 2004, 4, 383. (c) Hamley, I. W. Block Copolymers in Solution; Wiley: Chichester, 2005.

(538) Schlaad, H. Adv. Polym. Sci. 2006, 202, 53.

(539) (a) Burkoth, T. S.; Benzinger, T. L. S.; Jones, D. N. M.; Hallenga, K.; Meredith, S. C.; Lynn, D. G. J. Am. Chem. Soc. 1998, 120, 7655. (b) Burkoth, T. S.; Benzinger, T. L. S.; Urban, V.; Lynn, D. G.; Meredith, S. C.; Thiyagarajan, P. J. Am. Chem. Soc. 1999, 121, 7429. (c) Thiyagarajan, P.; Burkoth, T. S.; Urban, V.; Seifert, S.; Benzinger, T. L. S.; Morgan, D. M.; Gordon, D.; Meredith, S. C.; Lynn, D. G. J. Appl. Cryst. 2000, 33, 535.

(540) (a) Hamley , I. W.; Krysmann, M. J.; Castelletto, V.; Kelarakis, A.; Noirez, L.; Hule, R. A.; Pochan, D. Chem. Eur. J. 2008, 14, 11369. (b) Hamley , I. W.; Krysmann, M. J.; Castelletto, V.; Noirez, L. Adv. Mater. 2008, 20, 4394. (c) Castelletto, V.; Newby, G. E.; Zhu, Z.; Hamley , I. W.; Noirez, L. Langmuir 2010, 26, 9986.

(541) Castelletto, V.; Newby, G. E.; Hermida-Merino, D.; Hamley , I. W.; Liu, D.; Noirez, L. Polymer Chemistry 2010, 1, 453. 
(542) Castelletto, V.; McKendrick, J. M. E.; Hamley, I. W.; Cenker, C.; Olsson, U. Langmuir 2010, 26, 11624.

(543) Li, C.; Orbulescu, J.; Sui, G.; Leblanc, R. M. Langmuir 2004, 20, 8641.

(544) Deng, M. L.; Yu, D. F.; Hou, Y. B.; Wang, Y. L. J. Phys. Chem. B 2009, 113, 8539.

(545) He, C.; Han, Y.; Fan, Y.; Deng, M.; Wang, Y. Langmuir 2012, 28, 3391. 